\title{
STATE AND LOCAL REGULATION OF IMMIGRATION: THE NEED FOR A BILATERAL (RECIPROCAL) RATCHET
}

\author{
David P. Weber
}

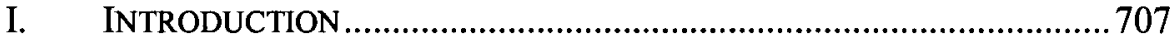

II. THE DEBATE OVER PREEMPTION IN IMMIGRATION .........................710

A. Preemption Defined ........................................................... 711

B. Immigration-Related Preemption............................................ 712

1. Historic Roots of Preemption......................................... 712

2. Modern Immigration Preemption Trends........................714

a. State Regulation of Immigration ............................... 714

b. Local Regulation of Immigration .............................. 715

i. Local Laws and Ordinances

Deemed Preempted ..............................................715

ii. Local Laws and Ordinances

Upheld .................................................................

3. Beyond E-Verify and Employment: S.B. 1070 and its Copycats ......................................................................720

4. 2011: The Supreme Court Acts.......................................721

a. Chamber of Commerce of the United States $v$.

b. Martinez v. Regents of the University of California.. 724

c. Hazleton v. Pennsylvania ...........................................727

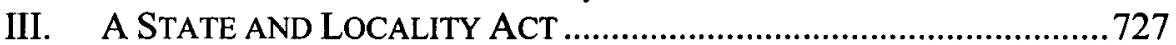

IV. PROPOSING A BILATERAL RATCHET ...........................................732

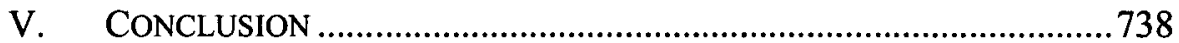

\section{INTRODUCTION}

The issue of federal preemption in the field of immigration has been swirling more and more strongly in academia, the courts, and even the public consciousness ${ }^{1}$ ever since De Canas v. Bica was decided in $1976{ }^{2}$

* Associate Professor at Creighton School of Law. The author would like to thank LatCrit for the opportunity to present this paper at the 2011 Annual South-North Exchange on Theory, Culture and Law (SNX) and his fellow panelists for the many useful comments received on the topic, and researcher Emma Nagengast for her helpful edits, comments and research assistance.

1. See, e.g., Rich Samp, Note to the DOJ: The Supreme Court is Watching You on Preemption, FORBES, May 27, 2011, available at http://blogs.forbes.com/docket/2011/05/27/note-todoj-the-supreme-court-is-watching-you-on-preemption.html (last visited Feb. 17, 2012).

2. De Canas v. Bica, 424 U.S. 351(1976). 
As stricter immigration control laws were enacted in the 1980s and 1990s, the debate on preemption has sharpened. ${ }^{3}$ Since then, the many laws passed in state and local jurisdictions have prompted an outpouring of literature on the topic of federal preemption (express or implied), ${ }^{4}$ local regulation of immigration, ${ }^{5}$ and the potentially discriminatory undertones that seem to pervade much of the debate. ${ }^{6}$

Arizona, though it receives the lion's share of coverage, is nowhere near alone in terms of states and cities opting to regulate immigration, whether directly or indirectly. ' In light of three 2011 Supreme Court decisions, Chamber of Commerce of the United States v. Whiting, ${ }^{8}$ Martinez v. Regents of the University of California, ${ }^{9}$ and Hazleton v. Pennsylvania, ${ }^{10}$

3. See generally Immigration Reform and Control Act (IRCA) of 1986, Pub. L. No. 99-603, 1986 U.S.C.C.A.N. (100 Stat.) 3359 (codified as amended scattered sections of 8 U.S.C.); Immigration Act of 1990, Pub. L. No. 101-649, 1990 U.S.C.C.A.N. (104 Stat.) 4978 (codified as amended scattered sections of 8 U.S.C.).

4. See, e.g., Clare Huntington, The Constitutional Dimension of Immigration Federalism, 61 VAND. L. ReV. 787 (Apr. 2008); Federal Statutes and Regulations, Federal Preemption of State Law, 114 HARV. L. REV. 339 (Nov. 2000); Gary Endelman \& Cynthia Juarez Lange, State Immigration Legislation and the Preemption Doctrine, 1968 PLI/CORP. 123 (Oct. 14-15 2008); Caleb Nelson, Preemption, 86 VA. L. REV. 225 (Mar. 2000).

5. See Immigrant Policy Project, National Conference of State Legislatures, available at http:/www.ncsl.org/default.aspx?tabid=19897 (last visited Feb 17, 2012) [hereinafter Immigrant Policy Project] (summarizing the immigration legislation that was enacted at the state level in 2009, 2010 , 2011 and noting that in the first quarter of 2011, legislators in all fifty states and Puerto Rico had introduced 1,538 proposals relating to immigrants and refugees).

6. See, e.g., Lindsay Nash, Expression by Ordinance: Preemption and Proxy in Local Legislation, 25 GEO. IMMIGR. L.J. 243, 247-51 (2011).

7. See L.B. 48, 102d Leg., 1st Sess. (Neb. 2011) (proposing Nebraska adopt the "Illegal Immigration Enforcement Act"); L.B. 569, 102d Leg., 1st Sess. (Neb. 2011) (requiring Nebraska employers to use the federal E-Verify system to verify employee's authorization to work in the United States); L. Res. 224, 100th Leg., 2d Sess. (Neb. 2007) (proposing Nebraska adopt the "Memoranda of Agreement, as authorized by section $287(\mathrm{~g})$ of the federal Illegal Immigration Reform and Immigrant Responsibility Act of 1996"). See also H.B. 7089, 113th Leg., Reg. Sess. (Fla. 2011); S.B. 104, 151st Gen. Assemb., Reg. Sess. (Ga. 2011); H.B. 1969, 97th Gen. Assemb., 1st Sess. (Ill. 2011); S.F. 102., 84th Gen. Assemb., 1st Sess. (lowa 2011); S.B. 6, 11 th Gen. Assemb., Reg. Sess. (Ky. 2011); H.B. 3341, 76th Leg., Reg. Sess. (Or. 2011); H.B. 1375, 82d Leg, Gen. Sess. (Tex. 2011) (prohibiting state or local authorities from adopting laws or ordinances that would restrict the enforcement of the federal immigration laws); Guest Worker Program Act, H.B. 116, 59th Leg., Gen. Sess. (Utah 2011); Utah Illegal Immigration Enforcement Act, H.B. 497, 59th Leg., Gen. Sess. (Utah 2011) (forming the Utah Compact addressing employment, identification, and verification).

8. Chamber of Com. of U.S. v. Whiting, 131 S. Ct. 1968, 1972 (2011) (upholding Arizona's law that, inter alia, mandates the use of E-Verify as well as provides penalties for those who employ unauthorized immigrants).

9. Martinez v. Regents of the Univ. of Cal, 131 S. Ct. 2961 (2011) (denying certiorari in a case challenging California's law granting in-state tuition to unauthorized immigrants that meet certain 
cases that seem to embrace a narrower interpretation of preemption, this essay proposes a bidirectional ratchet that would allow states and localities the ability to create more balanced immigrant legislation.

As it stands, even though states are directly prohibited from criminalizing immigration-related employment violations, ${ }^{11}$ states and localities are allowed to regulate immigration in pro-enforcement ways through the $287(\mathrm{~g})$ program $^{12}$ and Secure Communities; ${ }^{13}$ this trend has been popularized by Arizona and copied throughout the nation by enforcement through attrition--raising the cost of business for those who may utilize unauthorized immigrant labor (the latter attained largely through the threat of license revocation). ${ }^{14}$

This Article proposes a broader approach regarding pro-immigrant, sub-federal regulation in order to level the current legislative playing field. Under the current preemption approach, supported by many who reasonably fear unharnessed sub-federal discrimination against immigrants, Congress has granted states the right to act in this field exclusively in a proenforcement direction. Given the approach, regardless of the dubious legality of laws such as Arizona's S.B. 1070, Nebraska's L.B. 48, or Georgia's H.B. 87, the laws continue to proliferate. ${ }^{15}$ Rather than allow only pro-enforcement proposals, a more flexible approach in examining how to view preemption should be used to grant states the ability to generate more adaptable, varied approaches to the issue of unauthorized immigration. ${ }^{16}$

high school requirements). The case is important for preemption purposes (together with Whiting and Hazleton) as it establishes the Roberts Court's views on preemption. In Martinez, the question of preemption potentially loomed large given that 8 U.S.C. $\$ 1623$ (a) expressly provides that states cannot provide any post-secondary benefits based on residence within a State (or political subdivision). See Id.; 8 U.S.C. $\$ 1623(a)$.

10. City of Hazleton v. Lozano, 131 S. Ct. 2958, 2958 (2011) (remanding for reconsideration a Third Circuit decision that had struck down on preemption grounds a local ordinance that prohibited the hiring of and the renting of a dwelling place to unauthorized immigrants).

11. See Whiting, $131 \mathrm{~S}$. Ct. at 1973 (stating "Federal immigration law expressly preempts "any State or local law imposing civil or criminal sanctions (other than through licensing and similar laws) upon those who employ . . . unauthorized aliens."') (citing 8 U.S.C. $\S 1324 \mathrm{a}(\mathrm{h})(2)$ )).

12. See Immigration and Nationality Act, 8 U.S.C. $\$ 1357$ (g) (codifying the 287 (g) program).

13. See Secure Communities, U.S. IMMIGR. \& CUSTOMS ENFORCEMENT, available at http://www.ice.gov/secure_communities/ (last visited Feb. 8, 2012) (defining secure communities).

14. See S.B. 1070, 49th Leg., 2d Sess. (Ariz. 2010).

15. Ariz. S.B. 1070; Ga. S.B. 104; Neb. L.B. 48.

16. For example, Utah's recently passed law that would allow the state, in conjunction with the government of Mexico, to grant work permits to unauthorized immigrants within the state requires federal government preapproval, which preapproval does not appear to be forthcoming. See Utah Illegal Immigration Enforcement Act, Utah H.B. 497; Stephen Dinan, Holder Eyes Utah Guest-Worker Law, 
Part I of this essay briefly outlines the historical debate regarding the federal regulation of immigration, the federal preemption standard and then analyzes recent cases focused specifically on preemption. Part II of this essay focuses on several sub-federal regulations passed or proposed in Nebraska in 2010 and 2011 that are reflective of the greater debate raging nationwide. Finally, in light of three recent Supreme Court actions, Part III of this paper proposes a more progressive analysis regarding state and local regulation of immigration in order to grant states the ability to create and implement immigrant-positive legislation. ${ }^{17}$ Part III suggests a more flexible approach based partially on immigrant access to certain essential rights including employment, housing, transportation, education, and public services. The article concludes that a more progressive, pro-immigrant approach will level the legislative playing field and best serve state and federal interests in regulating immigration.

\section{THE DEBATE OVER PREEMPTION IN IMMIGRATION}

In May 2011, the Supreme Court announced its decision in Chamber of Commerce of the United States v. Whiting, ${ }^{18}$ upholding Arizona's 2007 law that allows the appropriate authorities in the state to suspend or revoke certain business licenses for those businesses who "knowingly or intentionally employ" unauthorized immigrants. ${ }^{19}$ The primary reason for upholding the law was the express language in the statute that allows states to impose sanctions "through licensing and similar laws" (hereinafter the "Savings Clause"), which have generally been regarded as traditional spheres of state regulation. ${ }^{20}$ Whiting must also be read in the context of the

WASH. TIMES, May 2, 2011, available at http://www.washingtontimes.com/news $/ 201$ 1/may/3/holdereyes-utah-guest-worker-law/?page=all\#pagebreak (last visited Feb. 8, 2012).

17. As it stands, the Whiting decision is at least partially at odds with the holdings in cases such as the Third Circuit's decision in Hazleton and the United States District Court for the Northern District of Texas' decision in Villas at Parkside Partners v. Farmer's Branch. See Hazleton, Pa., $131 \mathrm{~S}$. Ct. at 2958; Villas at Parkside Partners, et al. v. City of Farmer's Branch, Tex., 701 F. Supp. 2d 835,835 (N.D. Tex. 2010). Since 8 U.S.C. $\$ 1324 a(h)(2)$ contains a savings clause that only benefits increased enforcement regulations, the current statutory regime is, by its nature, pro-enforcement. A more flexible approach towards state regulation could at least allow more inclusive proposals. See 8 U.S.C. $\S$ $1324 \mathrm{a}(\mathrm{h})(2)(2006)$.

18. Whiting, $131 \mathrm{~S}$. Ct. at 1973.

19. ARIZ. REV. StAT. ANN. $\S \S 23-211,212,212.01$ (West 2010). This law is not to be confused with the more infamous Ariz. S.B. 1070 which is much broader in scope and purpose, and which was partially invalidated on preemption grounds by the U.S. Supreme Court in 2012. Arizona v. U.S., 132 U.S. 2492, 2497-98, 2510 (2012) (invalidating on preemption grounds provisions creating new state crimes for immigration violations). See also Ariz. S.B. 1070.

20. 8 U.S.C. § 1324a(h)(2). 
past thirty years of immigration-related preemption analysis, which appears to be trending away (albeit not uniformly so) from a broad, robust preemption framework. ${ }^{21}$ In turn, all immigration-related preemption analysis needs to be viewed in light of the historic trends regarding preemption analysis in general.

\section{A. Preemption Defined}

A brief recap of federal preemption law is necessary to understand its constitutional underpinnings prior to focusing in on immigration-specific application of the doctrine. Under federal law, there are generally two types of preemption: express and implied, and both types arise from either the Supremacy Clause of the U.S. Constitution or from language in a federal statute declaring preemptive effect. ${ }^{22}$ Simply stated, the Supremacy Clause prohibits any subfederal laws that "interfere with or are contrary to" federal law, ${ }^{23}$ whereas express preemption occurs when Congress, through statute, has explicitly held that any related subfederal laws are displaced. ${ }^{24}$

In contrast to express preemption, implied preemption can occur in one of two ways: field preemption or conflict preemption. One way in which field preemption occurs is where federal law is so extensive in an area as to have been deemed to 'occupy' a given subject matter exclusively. ${ }^{25}$ In those situations where federal law is "so pervasive as to make reasonable the inference that Congress left no room for the States to supplement it," subfederal laws will be struck down even if not in direct conflict with the federal statute in question. ${ }^{26}$ Other techniques that courts have used to find field preemption is whether "the federal interest in the field is sufficiently dominant"27 and whether "the object sought to be obtained by the federal law and the character of obligations imposed by it reveal the same purpose." ${ }^{28}$

21. See infra notes $32-40$ and accompanying text.

22. U.S. CONST. art. VI, cl. 2. See also English v. General Elec. Co., 496 U.S. 72, 78 (1990) (stating "Congress can define explicitly the extent to which its enactments preempt state law") (citing Shaw v. Delta Air Lines, Inc., 463 U.S. 85, 95-98 (1983)).

23. U.S. ConST. art. VI, cl. 2; English, 496 U.S. at 78.

24. English, 496 U.S. at 72.

25. Rice v. Santa Fe Elevator Corp., 331 U.S. 218, 230 (1947).

26. Rice, 331 U.S. at 230.

27. Schneidewind v. ANR Pipeline Co., 485 U.S. 293, 300 (1988).

28. Rice, 331 U.S. at 230. 
The other type of implied preemption is conflict preemption. ${ }^{29}$ Conflict preemption exists where a subfederal law "stands as an obstacle to the accomplishment and execution of the full purposes and objectives of Congress," or it is not possible for a party to comply with both the federal and subfederal law simultaneously. ${ }^{30}$ An immigration-related example would be localities enacting sanctuary-type ordinances to prevent or disrupt the removal of unauthorized immigrants. ${ }^{31}$

\section{B. Immigration-Related Preemption}

\section{Historic Roots of Preemption}

One reasonable conclusion after reviewing an immigration-related preemption analysis is that it strongly resembles the path of a river that has shifted dramatically and often unpredictably over time. Prior to discussing preemption, however, it is first necessary to get to the underlying authority to legislate. On that note, for the better part of the first one-hundred years of the United States, immigration law was largely ignored on a federal level. ${ }^{32}$ It was not until the late 1800 s and the foundational cases of Chae Chan Ping v. United States (The Chinese Exclusion Case), Fong Yue Ting v. United States, Ekiu v. United States, Henderson v. Mayor of City of New York, and Chy Lung v. Freeman that the Court acknowledged, quite forcefully, Congress' plenary and exclusive role in immigration. Following those cases and the need that some commentators have identified for uniform immigration laws and enforcement, ${ }^{33}$ it became apparent that a preemption challenge could easily lurk in the background anytime a state or locality were to pass a law related in some manner to immigration.

Though the potential for a preemption challenge exists in nearly all cases of subfederal regulation, it has not led to the ultimate conclusion that

29. See, e.g., Hines v. Davidowitz, 312 U.S. 52 (1941) (showing that one of the early conflict preemption cases happens to be immigration related). In 1939, Pennsylvania attempted to adopt an alien registration act requiring an identification card which the U.S. Supreme Court ultimately held was superseded and preempted by the federal Alien Registration Act which contained no identification card requirement. See Id. at 74 .

30. Hines, 312 U.S. at 67.

31. See generally United States v. Merkt, 794 F.2d 950 (5th Cir. 1986).

32. See generally John Higham, American Immigration Policy in Historical Perspective, 21 LAW \& CONTEMP. PROBS. 213 (1956).

33. See Huyen Pham, The Inherent Flaws in the Inherent Authority Position: Why Inviting Local Enforcement of Immigration Laws Violates the Constitution, 31 FLA. ST. U. L. REV. 965, 1003 (2004). 
these statutes and ordinances are preempted and unenforceable. ${ }^{34}$ In fact, some principled distinctions have arisen. In arguably the seminal case of modern preemption analysis for immigration law, the Court in De Canas $v$. Bica held that "the fact that aliens are the subject of a state statute does not render it a regulation of immigration, which is essentially a determination of who should or should not be admitted into the country, and the conditions under which a legal entrant may remain." ${ }^{35}$ The Court noted, "there would not appear to be a . . f federal interest in a situation in which the state law is fashioned to remedy local problems, and operates only on local employers, and only with respect to [those] individuals" the federal government has deemed unauthorized to work in the country. ${ }^{36}$

Even though statutorily overruled with the enactment of the Immigration Reform and Control Act (IRCA),${ }^{37}$ the essential holding in De Canas that laws that affect immigrants are not necessarily laws regulating immigration was not overturned. ${ }^{38}$ Indeed, other scholars have already observed some modern courts' reluctance to utilize implied theories of preemption where possible. ${ }^{39}$ Lawmakers and advocacy groups alike have latched on to preemption analyses and tea-leaf reading in an effort to enact or oppose local laws. ${ }^{40}$

34. See, e.g., Whiting, 131 S. Ct. at 1970 (concluding Arizona's "Legal Arizona's Worker's Act" was not preempted by federal law). See also ARIZ. REv. STAT. ANN. $\S \S 23-211,212,212.01$; De Canas, 424 U.S. at 351.

35. De Canas, 424 U.S. at 355.

36. Id.

37. Immigration Reform and Control Act, 8 U.S.C. $\$ \S 1160,1187,1188,1255(a), 1324 a$, 1324b, 1364, 1365 (1986) (addressing employment of unauthorized immigrants).

38. See Toll v. Moreno, 458 U.S. 1, 17 (1982) (holding a Maryland state university policy that prohibited certain non-immigrants and their dependants from acquiring in-state resident status violated the Supremacy Clause of the U.S. Constitution).

39. See, e.g., Cristina M. Rodriguez, The Significance of the Local in Immigration Regulation, 106 MICH. L. REV. 567 (2008).

40. See Appellant's Reply Brief at 15-16, Lozano v. City of Hazleton, 620 F.3d 170 (3rd Cir. 2010), Case No. 07-3531, 2008 WL 3989656 (arguing that the ordinances are not expressly preempted by stating that "[a]ppellees go to extraordinary lengths to twist this express permission into an express prohibition, resurrecting a far-fetched theory ... that 8 U.S.C. $\$ 1324 \mathrm{a}(\mathrm{h})(2)$ allows a city to impose licensing sanctions only after the employer has been tried in a federal criminal or civil proceeding and found to violate an IRCA provision") (citing Appellees' Brief at 75); Appellants' Reply Brief at 18, U.S. v. Arizona, 641 F.3d 339 (9th Cir. 2011) (No. 10-16645), 2010 WL 5162517, at18 (quoting "[n]or can the United States establish conflict preemption merely by demonstrating that there is a difference between state and federal law. The United States has not identified an actual conflict between section 5(C) and federal law because the United States has not shown that it was Congress' purpose to protect unauthorized employees from state sanctions." (citations omitted)). 


\section{Modern Immigration Preemption Trends}

As recent case law has unfolded at a dramatic pace in the latter half of the 2000 s and early 2010s, lawmakers have attempted various strategies to minimize the risk of a preemption battle. ${ }^{41}$ In light of this recent wave of subfederal litigation ${ }^{42}$ and the 2011 and 2012 Supreme Court actions on related cases, ${ }^{43}$ this author believes that another shift in immigration-related preemption is already underway. The most interesting aspect of the current shift is the potential extent to which states' roles in immigration may be enlarged.

\section{a. State Regulation of Immigration}

In 2007, Arizona passed a law requiring employers to use E-Verify ${ }^{44}$ to confirm an employee's work authorization, and it also contained penalties in the form of license revocations for employers who were not in compliance with federal prohibitions regarding the employment of unauthorized immigrants. While nowhere near as sweeping as the 2010 Arizona law known as S.B. 1070, the 2007 law was perceived as susceptible to a preemption challenge. ${ }^{45}$ As discussed in Part I.A.4.a., in 2011, the Supreme Court rejected the preemption argument for a few, very specific reasons. While newsworthy, the decision in Whiting was not unexpected as both the District Court and the Ninth Circuit had upheld the law, and while the differing policy arguments stressed by Justices Breyer ${ }^{46}$

41. See, e.g., Utah Illegal Immigration Enforcement Act, H.B. 497. See also supra notes 8, 10-11 and accompanying text.

42. See supra note 7.

43. See Regents of the Univ. of Cal., 131 S. Ct. at 2961; Whiting, 131 S. Ct. at 1968; Hazleton, Pa., 131 S. Ct. at 2958; Arizona, 132 U.S. 2492.

44. See ARIZ. REV. STAT. ANN. $\$ \S 23-211,23-214$. E-Verify was officially enacted under the title "Basic Pilot Program" and have been subject to fairly tumultuous treatment in its short history. See Illegal Immigration Reform and Immigrant Responsibility Act of 1996, Pub. L. No. 104-208, $\S 401-$ 05,110 Stat. $3009,3009-655$ to 3009-666. The law explicitly states that Congress could not mandate its use. 8 U.S.C. $\$ \S 1324 \mathrm{a}, 1324 \mathrm{~b}$.

45. See, e.g., Fawn Johnson, High Court Could Lean Toward States on Immigration, NAT'L J. DAILY (Dec. 9, 2010), available at http:/www.nationaljournal.com/high-court-could-lean-towardstates-on-immigration-20101208 (last visited Mar. 17, 2012); Stephen Dinan, U.S. Pushes E-verify for Hires Few Agencies Employ System, WASH. TIMES, Sept. 25, 2007, at 1.

46. Whiting, $131 \mathrm{~S}$. Ct. at 1987-97 (Breyer, J., dissenting) (reasoning, Congress did not intend its 'licensing' language to create so broad an exemption, for doing so would permit States to eviscerate the federal Act's pre-emption provision, indeed to subvert the Act itself, by undermining Congress' efforts 1) to protect lawful workers from national-origin-based discrimination and 2) to protect lawful employers against erroneous prosecution or punishment.). 
and Sotomayor ${ }^{47}$ discussing the delicate balance obtained by IRCA were compelling, they were insufficient to overcome what $\mathrm{J}$. Roberts saw as the plain language of the statute.

Interestingly, the preemption battle has not been solely waged by those in favor of pro-immigrant legislation. Martinez, where California granted in-state tuition to unauthorized immigrants based on the location of the high school from which they graduated, appears to go further than Whiting in that it arguably allows for state action that is perhaps beyond the language of the statute. ${ }^{48}$ The legislation in Martinez was challenged on preemption grounds ${ }^{49}$ for violating the federal prescription on benefits for unauthorized immigrants based on residence. ${ }^{50}$

In Martinez, arguments for express preemption, conflict preemption, and field preemption were unsuccessful in persuading the Court to intervene on preemption grounds and the California Supreme Court was likewise not persuaded that the law was preempted. ${ }^{51}$ Reading these cases together, ${ }^{52}$ it would appear that the Court is heading toward a narrower interpretation of preemption than had previously been applied using reasoning similar to that used in De Canas and that the regulations, while affecting immigrants, were not regulating immigration. ${ }^{53}$ While these two cases alone may not seem to advance De Canas very far, the Hazleton decision as well as the looming battle over S.B. 1070 may continue to see the Court's narrowing of the preemption battlefield in favor of finding a larger subfederal role in the debate.

\section{b. Local Regulation of Immigration}

\section{i. Local Laws and Ordinances Deemed Preempted}

As noted above, since the turn of the century, states and localities have begun to consider and enact legislation relating to immigrants and/or

47. Id. at 1998-2007 (Sotomayor, J., dissenting).

48. See Martinez v. The Regents of the Univ. of Cal., 241 P.3d 855, 861 (Cal. 2010).

49. Brief for Petitioner at *6-8, 16-20, Martinez, 241 P.3d at 855, 2011 WL 549168.

50. See Martinez, 241 P.3d at 860 . See also, 8 U.S.C. § 1623(a) (expressly prohibiting states from providing any post-secondary benefits based on residence).

51. See Martinez, 241 P.3d at 855, cert. denied, 131 S. Ct. 2961 (2011).

52. See Regents of the Univ. of Cal., 131 S. Ct. at 2961 ; Whiting, 131 S. Ct. at 1970 . See also De Canas, 424 U.S. at 355.

53. See Regents of the Univ. of Cal., 131 S. Ct. at 2961; Whiting, 131 S. Ct. at 1970. See also De Canas, 424 U.S. at 355. 
immigration on an increasingly frequent basis with mixed results. ${ }^{54}$ Recently three principal locales have tested the validity of these types of local ordinances: Texas, ${ }^{55}$ Missouri, ${ }^{56}$ and Pennsylvania. ${ }^{57}$ All three jurisdictions enacted similar statutes that attempted either to reduce the hiring of unauthorized immigrants or restrict the ability of unauthorized immigrants to rent housing, typically by relying on anti-harboring provisions, or both. ${ }^{58}$ The ordinances faced both preemption and due process challenges with mixed results as the Hazleton, Pennsylvania and Farmers Branch, Texas ordinances were initially struck down, and the Valley Park, Missouri ordinance was upheld. ${ }^{59}$

The Farmers Branch Ordinance purported to require residential occupancy licenses ${ }^{60}$ but relied heavily on classifications of eligibility for federal housing assistance. ${ }^{61}$ In its initial 2008 decision, the District Court struck down the ordinance on preemption and due process grounds. ${ }^{62}$ The city attempted to rectify the preemption and due process violations with a follow-up ordinance, which in addition to bearing striking resemblance to ordinances enacted by other localities, contains no references to eligibility for federal housing subsidies, incorporates local and judicial review mechanisms, and defers to federal law to determine authorized status. ${ }^{63}$

54. See Immigrant Policy Project, supra note 5 (noting the over 1,500 piece of subfederal immigrant-related legislation in the first quarter of 2011 , a significant increase over the 2010 numbers). See also, Roger Buddenberg, Immigration Ferment, OMAHA WORLD HERALD 4A, June 10, 2011 (noting that immigration-related bills had been introduced at a rate $30 \%$ higher than a year ago).

55. See Villas at Parkside Partners, 701 F. Supp. 2d at 835.

56. See Gray v. City of Valley Park, Mo., 567 F.3d 976 (8th Cir. 2009).

57. See Lozano v. City of Hazleton, 496 F.Supp. 2d 477 (M.D. Penn. 2007).

58. See Lozano v. City of Hazleton, 620 F.3d 170, 176 (3rd Cir. 2010) (discussing Hazleton's city ordinances: Illegal Immigration Relief Act Ordinance ("IIRAO"), Ordinance No. 2006-18, amended by Ordinance No. 2006-40, and Ordinance No. 2007-6 and the Rental Registration Ordinance ("RO") Ordinance No. 2006-13); Gray, 567 F.3d at 976 (stating "ordinance 1722 prohibits all business entities in Valley Park from knowingly employing unauthorized aliens"); Villas at Parkside Partners, 701 F. Supp. 2d at 839 (stating “Ordinance 2952 conditions residence in rental housing”).

59. See Id.

60. See Farmers Branch, Tex., Ordinance 2903 (May 12, 2007) (adopted by a citywide vote by a 2-1 margin to replace Ordinance 2892).

61. See Villas at Parkside Partners v. City of Farmers Branch, 577 F. Supp. 2d 858, 874-75 (N.D. Tex. 2008).

62. Id. at $875-77$.

63. See Farmers Branch, Tex., Ordinance 2952, $\$ 26-79(B), 26-119$ (2010) (stating that the purpose of the law was "to enact regulations that are harmonious with federal immigration law and which aid in its enforcement ... not . . to alter, supplant, disrupt, or interference with federal immigration law"). See also Villas at Parkside Partners, 701 F. Supp. $2 \mathrm{~d}$ at 841 (noting adoption of 
In preemption cases, courts utilize a presumption against preemption if they deem the field to be one in "which the States have traditionally occupied." ${ }^{164}$ In Farmers Branch, however, the court found the ordinance sufficiently dependent on federal immigration law so as to not merit the presumption. The District Court then struck down the Farmers Branch's ordinance on field preemption grounds. ${ }^{65}$ The court concluded the ordinance, while piggybacking on federal standards, "use[d] those classifications for purposes not authorized or contemplated by federal law." restrictions based on immigration classifications to directly alter the ability of individuals to remain present in the country, or, to put it another way, the ordinance improperly affected immigration and not just immigrants. ${ }^{67}$

Similar to Farmers Branch, the city of Hazleton, Pennsylvania also adopted an ordinance regulating unauthorized immigrants, though the Hazleton ordinance went further and also attempted to sanction employers for hiring unauthorized immigrants and it required implementation of $\mathrm{E}$ Verify ${ }^{68}$ The ordinance also adopted federal definitions for lawful presence and required the final determination of authorized presence to be verified by the federal government. ${ }^{69}$ Upon challenge, the District Court of Pennsylvania struck down the ordinance in its entirety on grounds of preemption, ${ }^{70}$ and that ruling was upheld at the Third Circuit. ${ }^{71}$

Though it was ultimately remanded for reconsideration, the Third Circuit's decision in Hazleton could have been viewed as a circuit-level

federal definitions, deference to federal determination of immigration status and citation to antiharboring provisions).

64. U.S. v. Locke, 529 U.S. 89, 108 (2000).

65. See Villas at Parkside Partners, 701 F. Supp. at 841 (citing Villas at Parkside Partners $v$. City of Farmers Branch, 577 F. Supp.2d 858, 866-67 (N.D. Tex. 2008)).

66. Id. at 855 .

67. Id. (citing De Canas, 424 U.S. at 358).

68. See Hazleton, Pa., Illegal Immigration Relief Act Ordinance (IIRAO), Ordinance 2006-18 (Sept. 8, 2006), available at http:/www.smalltowndefenders.com/090806/200618\%20_Illegal\%20Alien\%20Immigration\%20Relief\%20Act.pdf (last visited Mar. 3, 2012) [hereinafter IIRAO, Ordinance 2006-18]. Like the Farmers Branch ordinance, IIRAO included recitals regarding the city's authority to regulate local housing and employment insofar as it relates to the health, safety, and welfare of the city's residents. Id. § 2. See also Farmers Branch, Tex., Ordinance 2903 (May 12, 2007).

69. See IIRAO, Ordinance 2006-18, supra note $69, \S 3 \mathrm{E}$ (defining "unlawful worker" as "a person who does not have the legal right or authorization to work due to an impediment in any provision of federal, state or local law, including but not limited to a minor disqualified by nonage, or an unauthorized alien as defined by United States Code Title 8, subsection 1324a(h)(3)").

70. See Lozano, 496 F.Supp. $2 \mathrm{~d}$ at 477.

71. See City of Hazleton, $620 \mathrm{~F} .3 \mathrm{~d}$ at 170 (affirming in part and vacating in part). 
rebuke to any similar locally-driven, anti-immigrant legislation. ${ }^{72}$ The decision noted with favor the outcome in Farmers Branch, and refuted the results in Chicanos por la Causa, ${ }^{73}$ requiring the implementation of $\mathrm{E}$ Verify and Valley Park, involving a very similar ordinance where the court had struck down only the tenancy portion of the law. ${ }^{74}$ Additionally, when deciding the case, the court had the benefit of having the Ninth Circuit's decision in Chicanos por la Causa (restyled as Whiting) in hand.

In discussing express preemption in the employer context, and foreseeing the decision in Whiting, the Third Circuit looked closely at the Savings Clause. Ultimately the court held that the revocation of a business license for violating the unauthorized immigrant hiring prohibition fell squarely within the protection of the Savings Clause. ${ }^{75}$ However, on implied preemption grounds, the court held that the ordinance greatly disrupted the delicate balance between deterring employment of unauthorized immigrants and burdening employers and therefore stood as an obstacle to compliance with federal immigration law. ${ }^{76}$

In conclusion, the Third Circuit held that both the employment and rental portions of the ordinance were preempted. The employer provisions were struck down on an implied preemption theory as the court found the city created an impermissible, alternate system of compliance for employers and landlords, which, if allowed, could lead to an even greater number of states and localities implementing similar legislation leading to a

72. See Id. While the U.S. Supreme Court vacated and remanded the decision for reconsideration, other recent decisions similarly remanded were later reaffirmed by the Third Circuit. See, e.g., Abu-Jamal v. Sec'y, Pen. Dept. of Corrs., 643 F.3d 370 (2011); Kindler v. Horn, 642 F.3d 398 (2011), aff'd in part, rev'd in part, U.S. v. Bennett, 427 Fed.Appx. 228 (3d Cir. C.A. 2011).

73. See Chicanos Por La Causa, Inc. v. Napolitano, 544 F.3d 976 (9th Cir. 2009), opinion amended and superseded on denial of reh'g, 558 F.3d 856 (9th Cir. 2009), aff'd sub nom. Whiting, 131 S. Ct. at 1968; Arizona Contractors Ass'n, Inc. v. Candelaria, 534 F. Supp. 2d 1036, 1046 (D. Ariz. 2008), aff'd sub nom. Chicanos Por La Causa, Inc.,544 F.3d at 976, opinion amended and superseded on denial of reh'g, 558 F.3d 856 (9th Cir. 2009), aff'd sub nom. Whiting, 131 S. Ct. at 1968, and aff'd sub nom. Chicanos Por La Causa, Inc., 558 F.3d at 856, aff'd sub nom. Whiting, 131 S. Ct. at 1968.

74. City of Hazleton, 620 F.3d at 216, 218 (noting "[t]hese decisions, however, fail to afford proper weight to the purposes underlying Congress's decision to retain E-Verify as a voluntary program," "were likely to increase discrimination, and would contravene IRCA absent an antidiscrimination component," and "undervalue the emphasis Congress placed on preventing discrimination ....").

75. See Id. at 238. The Third Circuit did note as "very troubling" the argument that the "Findings and Declaration of Purpose" of the ordinance made no reference to licensing so that the law should be seen more properly as a "scheme intended to regulate immigration." Id. at $209 \mathrm{n} .31$.

76. Id. at 210-13 (noting also fewer procedural safeguards for violators under the ordinance). 
compliance morass for employers. ${ }^{77}$ The city fared even worse on the rental portion of the ordinance as the court found such regulation directly in opposition to the holding in De Canas as it was specifically aimed at regulating "who may live in Hazelton based on immigration status ...." While these two cases were among the most prominent of the cases where courts struck down local ordinances on preemption grounds, they were not the only ones. A law requiring employers to verify the work authorization of independent contracts, which goes beyond the scope of IRCA, also created a private cause of action against employers that hire unauthorized immigrants, ${ }^{79}$ was struck down by the Tenth Circuit on preemption grounds,${ }^{80}$ and a housing ordinance similar to the Hazleton ordinances was struck down on preemption grounds in California. ${ }^{81}$

\section{ii. Local Laws and Ordinances Upheld}

In stark contrast to the decisions in Hazleton and Farmers Branch, Valley Park was treated quite differently-at least pertaining to the employer and E-Verify portions of the ordinances, and it represents a case where a local ordinance touching on immigrant-related regulation was not struck down. ${ }^{82}$ In Valley Park, after briefly acknowledging the fact that the presumption against preemption would apply in the case, the court quickly resolved the express preemption question relying primarily on the plain

77. See Id. at 213-16. In the employer regulation context, the Third Circuit noted several conflicts including:

1) Creating an alternative mechanism for complaint investigation;

2) Fewer procedural protections;

3) Effectively eliminating the I-9 safe harbor by "coerc[ing]" the use of E-

Verify; and

4) Increasing the burden on interstate employers.

Id. (noting that such a patchwork of laws "could not possibly be in greater conflict with Congress's intent for its carefully crafted prosecution and adjudication system to minimize the burden imposed on employers) (citing Rowe v. N.H. Motor Transp. Ass'n, 552 U.S. 364, 373 (2008)).

78. City of Hazleton, 620 F.3d at 219-20 (emphasis in original).

79. A similar provision is found in SB 1070. See Ariz. S.B. 1070.

80. See Chamber of Com. of the U.S. v. Edmondson, 594 F.3d 742, 750 (10th Cir. 2010).

81. See Garret v. City of Escondido, 465 F.Supp.2d 1043, 1057 (S.D. Cal. 2006).

82. See Gray v. City of Valley Park, No. 4:07CV00881 ERW, 2008 WL 294294, at 14 (E.D. Mo. 2008). While Valley Park initially had a rental ordinance in addition to an employment ordinance, it voluntarily repealed the rental ordinance just months after its adoption. See Id. at 1. See also Valley Park, Mo. Mun. Ordinance 1721 (Feb. 2007). In the Valley Park case, the District Court "respectfully note[d] that the [Hazleton] decision [was] not binding," and would therefore independently evaluate the preemption arguments. See City of Valley Park, 2008 WL 294294, at10 n.13. 
language of the statute and the Savings Clause. ${ }^{83}$ The court likewise dealt summarily with the implied field preemption argument noting that it "required little discussion" as the Savings Clause evinced a federal intent to allow for some subfederal licensing regulations. ${ }^{84}$ However, while examining somewhat shallowly the first two claims of preemption, the court provided extensive commentary regarding implied conflict preemption. ${ }^{85}$ Where the District Court and Third Circuit in Hazleton had found numerous issues of potential conflict between federal law and very similar language in a separate local ordinance, ${ }^{86}$ the Valley Park court found none, though it did so in a fairly circuitous fashion. ${ }^{87}$

In contrast to what may be considered a more minor conflict, the larger area of concern in terms of preemption in Valley Park should have been the displacement of the balancing of burdens established by IRCA and extended by IIRIRA. In that regard, very little was said in a preemption context as the court addressed such concerns by framing them as equal protection based, and as to such discrimination, "little discussion is required." ${ }^{88}$ Where the court said more, however, was in the analysis of the E-Verify provisions, where its decision was subsequently validated in Whiting. ${ }^{89}$

\section{Beyond E-Verify and Employment: S.B. 1070 and its Copycats}

In the recent local/national argument regarding immigration, no law has caused a larger outcry than Arizona's S.B. $1070{ }^{90}$ S.B. 1070, upheld in part and invalidated in part, purported to greatly expand state involvement in immigration-related affairs by requiring local law enforcement to participate with federal officials, providing individuals a private cause of action to sue the government for failure to enforce the pro-restriction laws, creating additional state crimes for employment (by employer and

83. See City of Valley Park, 2008 WL 294294, at 10 (stating that "the question for the Court is solely whether the ordinance is a licensing or other similar law.").

84. See Id. at 13.

85. See Id. at 13-19.

86. See supra notes 68-78.

87. See City Valley Park, 2008 WL 294294, at *13-19 (discussing preemption arguments). In responding to the alleged conflict between the federal exemption removing domestic workers from the definition of employee and the Valley Park ordinance which arguably would include a domestic worker in its definition, the court held that "the small exception that is created[] is not sufficient to create a conflict between Congressional intent and the local ordinance." Id. at 14.

88. See Id. at 19.

89. See Id. at 16. See also Whiting, 131 S. Ct. at 1968.

90. Ariz. S.B. 1070. 
employee) and failure to carry immigrant registration papers, increasing the possibilities for state law enforcement to determine immigrant status, and allowing for warrantless arrests where probable cause exists to believe the immigrant is unlawfully present in the United States. ${ }^{91}$

The potential impact of S.B. 1070 is beyond the scope of this essay other than that of a brief mention. While the most immigrant-offensive provisions of the law were struck down on preemption grounds, the arguments by the federal government in contesting the law were focused on implied grounds of conflict and field preemption rather than express preemption. While some of the enjoined provisions likely do not rise to the De Canas level of immigration regulation, they appear to clearly rise to the Hines level of interfering with or conflicting with a comprehensive federal immigration scheme. ${ }^{92}$

Therefore, under current law, assuming that the precedent in Hines continues to be valid, S.B. 1070 and similar laws that attempt to expand states' participation and criminalize behavior already considered under the federal regulatory framework should continue to be struck down. ${ }^{93}$ The problem, of course, is that the states continue to act in pro-enforcement manners without oversight and no countervailing opportunities for more sympathetic states exist. The 2011 and 2012 decisions sharpen the focus of current immigration preemption law, but still leaves the balance tilted heavily on the side of enforcement.

\section{2011: The Supreme Court Acts}

Prior to the summer of 2011 , it was easy enough to simply recognize the varying results that occurred in the ongoing preemption battle in Hazleton (Third Circuit), Farmers Branch (Fifth Circuit), Escondido (Ninth Circuit), Arizona (Ninth Circuit), and Oklahoma (Tenth Circuit) as the origin of a healthy split among the circuits. ${ }^{94}$ However, given the three recent Supreme Court actions, at least some of the splits are being actively resolved. $^{95}$

91. See, e.g., ARIZ. REV. STAT. ANN. §§11-1051 et al., 13-1509, 13-2319, 13-2928, 13-2929, 13-3883, 23-212, 23-214.

92. See Arizona, 703 F. Supp. 2d at 999.

93. Indeed, Georgia's new law has already been federally enjoined. See Ga. Latino Alliance for Hum. Rts. v. Deal, 793 F. Supp. 2d 1317, 1322 (N.D. Ga. 2011) (granting the plaintiff's motion for preliminary injunction).

94. See Lozano, 620 F.3d at 170; Villas at Parkside Partners, 701 F. Supp. 2d at 835; Garrett, 465 F. Supp. 2d at 1043; Arizona, 641 F.3d at 339; Edmondson, 594 F.3d at 742.

95. See Regents of the Univ. of Cal., 131 S. Ct. at 2961; Whiting, 131 S. Ct. at 1970; Hazleton, Pa., 131 S. Ct. at 2958. 


\section{a. Chamber of Commerce of the United States $v$. Whiting}

First, we should begin with a descriptive discussion of any split that Whiting does resolve. In reaffirming the Ninth Circuit's decision by a 5-3 margin, ${ }^{96}$ the Court affirmed Arizona's right to enact licensing laws that, but for the Savings Clause, would have otherwise been preempted under federal immigration law. ${ }^{97}$ Unsurprisingly, the Court drew heavily on language from $\mathrm{De}$ Canas, which noted that the "States possess broad authority under their police powers to regulate the employment relationship to protect workers within the State," and that preventing unauthorized employment "is certainly within the mainstream of [the State's] police power....".98

Of some import to the outcome of Whiting is that Arizona deferred to the federal government's determination of whether or not an immigrant was authorized for employment prior to imposing sanctions in the form of license suspension or revocation, and largely adopted a federal definition of "license," and deferred to federal determinations regarding immigration status." While noting the definition of "license"100 in construing the Savings Clause, the Court found the Arizona statute, as a matter of "common sense" to fall within that definition. Not unexpectedly, the Court affirmed the Ninth Circuit's decision that the licensing law did fall within the Savings Clause under the plain language. However, the more interesting issue has always been whether, even assuming the applicability of the Savings Clause, there would be grounds for implied preemption

96. See Whiting, 131 S. Ct. at 1968. See also Nina Totenberd \& Ari Shapiro, Kagan Recused From Pending Supreme Court Cases, NPR (Sept. 29, 2010), available at http:/www.npr.org/templates/story/story.php?storyld=130205734 (last visited Mar. 3, 2012) (stating that $\mathrm{J}$. Kagan recused herself from the decision given her role as solicitor general prior to her confirmation).

97. See Whiting, $131 \mathrm{~S}$. Ct. at 1970 (stating that federal immigration law now expressly preempts civil or criminal sanctions imposed on employers other than those allowed under the Savings Clause). See also 8 U.S.C. $\S 1324 \mathrm{a}(\mathrm{h})(2)$.

98. Whiting, $131 \mathrm{~S}$. Ct. at 1974 (citing De Canas, 424 U.S. at 356, 360).

99. See ARIZ. REV. STAT. ANN. $\$$ 23-211 (defining "unauthorized alien" as "an alien who does not have the legal right or authorization under federal law to work in the United States as described in 8 United States Code $\S 1324 \mathrm{a}(\mathrm{h})(3) ")$. See also ARIz. REV. STAT. ANN $\S 23-214 ;$ Whiting, 131 S. Ct. at $1973,1975,1982-84$.

100. See Whiting, $131 \mathrm{~S}$. Ct. at 1978 (stating "the Arizona law defines 'license' as 'any agency permit, certificate, approval, registration, charter or similar form of authorization that is required by law and that is issued by any agency for the purposes of operating a business in' the State.") (citing ARIZ. REV. STAT. $§ 23-211(9)(a))$. Further, "[t]hat definition largely parrots the definition of 'license' that Congress codified in the Administrative Procedure Act." Whiting, 131 S. Ct. at 1978. See also 5 U.S.C. $\S 551(8)$ ("'[L]icense' includes the whole or a part of an agency permit, certificate, approval, registration, charter, membership, statutory exemption or other form of permission"). 
given the plenary power over immigration, and what some commentators have argued as the need for uniformity. ${ }^{101}$

Largely dismissing arguments that Congress had achieved a careful balance in its drafting of immigration laws, ${ }^{102}$ the Court issued an unambiguous decision, at least insofar as licensing laws and mandatory implementation of E-Verify is concerned. ${ }^{103}$ Utilizing analyses similar to the Ninth Circuit, the Court reasoned that the very fact that Congress provided a mechanism for some subfederal regulation under the guise of licensing laws strongly cuts against the argument that Congress intended to occupy the entire field of immigration. ${ }^{104}$ Additionally, the Court bypassed the plenary power issue when it framed the law as the "[r]egulat[ion] [of] in-states businesses through licensing," a traditional area of state regulation. ${ }^{105}$ With that framework adopted, the preemption analysis that followed was straightforward. The Court decided that Congress expressly provided a mechanism for states to impose sanctions through licensing laws, that the statute in question in Arizona acted to complement rather than supplement federal prohibitions on the employment of unauthorized immigrants, and therefore, no express or implied conflict existed. ${ }^{106}$

The E-Verify analysis was likewise straightforward and followed the premises that:

1) Federal law only prohibited a federal mandate of the EVerify program; ${ }^{10}$

2) E-Verify was made available by Congress for use by all the states; ${ }^{108}$

3) Arizona thereafter voluntarily adopted its E-Verify own mandate. ${ }^{109}$

101. See Whiting, 131 S. Ct. at 1990 (Breyer, J., dissenting) (objecting to the loss of "one centralized enforcement scheme"). See also Pham, supra note 33.

102. Whiting, $131 \mathrm{~S}$. Ct. at $1990-92$ (Breyer, J., dissenting) (highlighting the multiple ways in which the competing objectives of enforcing prohibitions against unauthorized hiring and limiting burdens on employers are balanced).

103. Id. at 1972.

104. Id. at 1971.

105. Id. at 1983.

106. See Id. at 1971 (noting that petitioners seeking to show preemption on the grounds of conflicting purposes need to meet a high threshold).

107. Whiting, $131 \mathrm{~S}$. Ct. at 1975 ("[T]he [Secretary of Homeland Security] may not require any person or other entity to participate in a pilot program.") (citing IIRIRA § 402(a), 110 Stat. 3009-656).

108. Id. at 1986 (" $[\mathrm{I}] \mathrm{n} 2003$ Congress required that E-Verify be made available in all 50 states. 117 Stat. 1944; IIRIRA § 401(c)(1), 110 Stat. 3009-656.”). 
While arguments as to the efficacy of E-Verify are legitimate, ${ }^{110}$ those arguments lack legal grounds on which to challenge the law. The only question to be resolved, which the Court answered in the affirmative, was whether Arizona's actions were within the range of acceptable activity. ${ }^{111}$

Therefore, for preemption purposes, Whiting establishes two important precedents. First, subfederal regulation operates in a sphere traditionally reserved to the states concerning valid licensing issues, and not regulating immigration in the De Canas sense is valid under the Savings Clause. Second, subfederal legislation mandating the use of E-Verify, provided that it does not depart from federal standards and definitions, presents no conflict and is likewise enforceable. The implication of both conclusions will be discussed further in Part III.

\section{b. Martinez v. Regents of the University of California}

While the Supreme Court's pronouncement in denying certiorari in Martinez was much briefer than its decision in Whiting, the impact on the current preemption framework may be sizeable. ${ }^{112}$ As mentioned above, in Martinez, ${ }^{113}$ the State of California enacted a law that purported to provide any student, regardless of immigration status, who met certain school-based attendance and graduation criteria, the opportunity to attend California's public universities while paying in-state tuition. ${ }^{114}$

Though not directly obvious on its face, there is a strong similarity between Martinez and Whiting.

Both cases turn on a narrow interpretation of the preemption doctrine when faced with specific statutory language. In Whiting, the Savings Clause was used in a traditional way, with the states reserving the ability to act in a proscribed, narrow manner. While the dissenting Justices argued

109. Id. at 1985 (noting that the federal government itself had argued in 2008, in regard to Arizona's law, "this is permissible because the State of Arizona is not the Secretary of Homeland Security") (emphasis in original) (citing Defendants' Reply Memorandum in Support of Their Motion for Summary Judgment (citation omitted)).

110. See E-Verify Statistics and Reports, U.S. CITIZENSHIP \& IMMIGR. SERV, available at http:/www.uscis.gov/portal/site/uscis/menuitem.5af9bb95919f35e66f614176543f6dla/?vgnextchannel= a 16988e60a4051 10VgnVCM1000004718190aRCRD\&vgnextoid=f82d8557a487al 10VgnVCM100000 4718190aRCRD (last updated June 7, 2011) (last visited Mar. 3, 2012) (showing that in Fiscal Year 2010 , almost 47,000 work authorized individuals (citizens and non-citizens alike) received an incorrect initial non-confirmation of work authorization).

111. See Whiting, $131 \mathrm{~S}$. Ct at 1987.

112. See Regents of the Univ. of Cal., 131 S. Ct. at 2961.

113. See Martinez, 241 P.3d at 861.

114. Id. (citing CAL. EDUC. CODE $§ 68130.5$ (West 2001)). 
for a narrower definition of "license" and a corresponding broader application of the preemption doctrine, the Court was not swayed by what it saw as a plain language solution. ${ }^{115}$ Likewise in Martinez, though ironically with the parties' positions reversed, ${ }^{116}$ the California Supreme Court adopted a plain language reading of a federal statute that also tended to narrow the reach of preemption arguments.

As noted, federal law prohibits states from providing unauthorized immigrants benefits "on the basis of residence within a state . . . for any postsecondary education benefit ..." unless the citizen or national of any state is eligible for said benefit without regard to state residency. ${ }^{117}$ In order to avoid that proscription, California conditioned the grant of in-state tuition on the requirements that students have attended high school in California for no less than three years and graduated from a California high school. ${ }^{18}$ In essence, the state uses the locale of the high school as a proxy for determining the residence of the student in deciding who should receive the benefits of in-state tuition. ${ }^{119}$ The California Supreme Court noted a correlation between the population of students who would likely qualify under the benefit statute and those who were in fact residents of the state, but held that as the criteria for both are clearly different, the proxy was not improper. ${ }^{120}$

As the language of the federal statute only prohibits benefits based on an individual's state residence, under a plain language analysis, the California law does not run afoul of that prohibition and thereby avoids express preemption. ${ }^{121}$ Beginning with the plain language approach ${ }^{122}$ and

\section{See Whiting, $131 \mathrm{~S} . \mathrm{Ct}$ at 1988.}

116. In Martinez, the parties seeking to strike down the statute as granting unlawful benefits to unauthorized immigrants argued for a broader interpretation of the language and resorted to arguments of legislative history and purpose. See Martinez, 214 P.3d at 855, 862-66. On the contrary, it was the party seeking to have the pro-enforcement Arizona law declared invalid due to preemption that relied on arguments of history and purpose. See Id.

117. See 8 U.S.C. $\$ 1623(a)$.

118. See CAL. EdUC. CODE $\$ 68130.5$.

119. See Id. The California Court of Appeal felt the proxy was improper as a de facto residence requirement. See Martinez v. Regents of Univ. of Cal., 83 Cal.Rptr.3d 518, 531, 540 (Cal. Ct. App. 2008), review granted and opinion superseded, 198 P.3d 1 (Cal. 2008), rev'd sub nom. Martinez, 241 P.3d at 855 .

120. See Martinez, 241 P.3d at 864 (explicitly disclaiming the criteria as a de facto or surrogate residency requirement, such disclaimer was unnecessary given the court's analysis that the federal statute in question prohibited benefits based on residency, "not some form of surrogate for residence ....").

121. See Id. at 863-64 (noting that the statute cannot logically be read to rely on residence as a criteria given "that many nonresidents may qualify for it") (emphasis in original). The court further noted that had Congress intended to prohibit any post-secondary benefits for unauthorized immigrants, it could have done so easily. Id. at 864 . 
the express preemption analysis above, the preemption argument in Martinez nearly mirrors the one made in Whiting regarding the supremacy of federal law in the United States when dealing with immigration. ${ }^{123}$ Working through the rest of the standard preemption framework: implied conflict (and/or obstacle) and field preemption, the discussion followed a similar path.

However, in Martinez, the California Supreme Court was explicit in stating that it was apparent the state was attempting to enact legislation to give a prohibited benefit to unauthorized immigrants. ${ }^{124}$ Even assuming that such a proposition was correct, the court noted for the purposes of express preemption analysis, "nothing is legally wrong with the legislature's attempt to avoid [the federal prohibition on postsecondary benefits]." 225 It is a closer question, however, whether such intent may create a conflict or obstacle to "the accomplishment and execution of the full purposes and objectives of Congress.",126

The legal hurdle faced by the opponents of the law is that the federal law did include an express preemption provision, one which did not cover the conduct/law at hand. As the Supreme Court has held that inclusion of an express preemption clause implies that Congress did not intend to preempt matters outside of its scope, ${ }^{127}$ the argument regarding the state legislature's intent, proper or not, is legally insufficient provided that the state legislature's actions fall outside the scope of the preemption clause. ${ }^{128}$ Additionally, any field preemption argument is likewise undercut by a limited express preemption clause given that any limits imply a window for state action which in turn strongly suggests that Congress did not intend to "occupy the field fully." 129

122. See Id. at 864-66 (discussing potentially adverse legislative history that conflicted with the actual language of the statute).

123. See Id. at 861 . See also Whiting, $131 \mathrm{~S}$. Ct. at 1975. In Martinez, however, the court declined to address the presumption question noting that it was immaterial to the ultimate outcome. See Martinez, 241 P.3d at 862.

124. See Martinez, 241 P.3d at 866.

125. Id.

126. Hines, 312 U.S. at 67 (citing Cf. Savage v. Jones, 225 U.S. 501,533 (1912)).

127. See Freightliner Corp. v. Myrick, 514 U.S. 280, 288 (1995).

128. See Martinez, 241 P.3d at 868.

129. Id. 


\section{c. Hazleton v. Pennsylvania}

The final case in the 2011 Supreme Court preemption trilogy, handed down on the same day as Martinez, is Hazleton. ${ }^{130}$ In a two sentence opinion, the Court granted certiorari and then vacated the decision of the Third Circuit and remanded the decision for further consideration in light of its decision in Whiting. ${ }^{131}$ While much or little could be made of the decision at this time, it does appear that it will be increasingly difficult for the opponents of the employment portion of Hazleton's ordinance to again be successful following the decision in Whiting. In fact, the major takeaway may be that the Court would like to see the preemption framework continue to be unified regarding employment provisions under the Savings Clause and the use of E-Verify, while likely also seeing a cohesive, circuit-level consensus emerge on the rental ordinances as no jurisdiction has upheld that type of subfederal regulation.

In fact, this dichotomy between employment and housing seems to be precisely what the Court has established this term. In clarifying the scope and applicability of the Savings Clause and the limits of implied preemption, the Court has seemingly returned to a more robust De Canas analysis that arguably narrows the grounds for implied preemption when analyzing subfederal regulations that directly or indirectly affect immigration. More troubling for many localities, however, is probably the question "What's next?" By refusing to provide a definitive answer on the housing ordinances, and given the fact that many localities enact employment and housing ordinances together, it is foreseeable that much litigation remains to be had in this context.

\section{A STATE AND LOCALITY ACT}

Although Arizona and now Utah, ${ }^{132}$ Georgia, ${ }^{133}$ and Alabama ${ }^{134}$ (among others) have stolen the spotlight, many states and cities across the country have been busy considering and enacting immigration-related laws. ${ }^{135}$ The State of Nebraska, in the geographic center of the country, ${ }^{136}$

130. See City of Hazleton, 620 F.3d at 170.

131. See Regents of the Univ. of Cal., 131 S. Ct. at 2961.

132. Utah Illegal Immigration Enforcement Act, Utah H.B. 497.

133. H.B. 87, 151st Gen. Assemb., Reg. Sess. (Ga. 2011).

134. H.B. 56, Reg. Sess. (Ala. 2011).

135. See Liz Goodwin, Report: 25 States Considering Arizona-Style Immigration Laws, YAHOO! NEWS (Oct. 28, 2010), http://news.yahoo.com/s/yblog_upshot/20101028/us_yblog_upshot/ report-25-states-are-considering-arizona-style-immigration-laws (last visited Mar. 3, 2012); John Miller, Twenty Other States Considering Copying Arizona Immigration Law, Houghington Post, June 25, 
has been no exception. For the purpose of this Article, two principal laws will be mentioned, a state-level modified version of Arizona's S.B. $1070^{137}$ and a local ordinance adopted by referendum and modeled on the Hazleton ordinance prohibiting employment of unauthorized immigrants and requiring "occupancy permits" to rent dwelling space. ${ }^{138}$

Prior to discussing these laws and partly to explain their impetus, I would like to note a few salient facts about the State of Nebraska and the town of Fremont, which enacted the ordinance in rural Nebraska. Nebraska's Hispanic population is approximately $8.4 \%$ of the population (compared to $15.8 \%$ of the overall U.S. population); however, while Nebraska's overall Hispanic population is below the national average, the 2010 Census indicated a ten-year growth rate of $77 \%$ in that population. ${ }^{139}$ Showing even greater demographic change is the percentage of children immigrants in 1990 as compared with $2005-2006{ }^{140}$ The percentage of immigrant children in $2005-2006$ was $11 \%$, a four-fold increase over that same number in $1990 .^{141}$

2010, available at http://www.huffingtonpost.com/2010/06/25/twenty-other-states-consi_n_ 626095.html (last visited Mar. 3, 2012).

136. Through amateur analysis using an old Rand-McNally atlas, the author has determined that the state of Nebraska, for example, lies approximately 1350 miles from the west coast, 1000 miles from the southern border, 1250 miles from the east coast, and a mere 400 miles from the northern border making it one of if not the state furthest from any international border.

137. L.B. 48 , the bill modeled on S.B. 1070 , failed to advance of committee discussions, for a number of reasons, not the least of which were the questionability of its validity, and the cost involved in defending such a statute. See Neb. L.B. 48. See generally Kevin O'Hanlon, Arizona-Style Immigration Measured Vetted in Overflow Public Hearing, LINCOLN J. STAR, Mar. 2, 2011, available at $\mathrm{http} / /$ journalstar.com/news/unicameral/article_717ddfa5-d555-5550-be0d-81d3271fff8.html (last visited Mar. 3, 2012).

138. See Fremont, Neb., Ordinance 5165 (June 21, 2010); Cindy Gonzalez, Fremont in the Spotlight, OMAHA WORLD Herald, Mar. 30, 2010, available at http://www.omaha.com/ article/20100530/NEWS01/705309883 (last visited Mar. 3, 2012) (explaining that in its initial appearance, the Fremont Ordinance was defeated at the city council level and that following its defeat, supporters of the ordinance were able to obtain sufficient signatures to have the matter decided as a referendum); Cindy Gonzalez, Fremont Weighs Tax Hike to Defend Law - The Public Can Comment on a Plan to Pay for Costs Related to the Immigration Ordinance, OMAHA WORLD HeRald, Aug. 23, 2010 , at $01 \mathrm{~B}$ (explaining that the residents approved the Ordinance overwhelmingly by a $2-1$ vote, while knowing beforehand that an affirmative vote was going to result in higher property taxes of approximately $18 \%$ ).

139. See Sharon R. Ennis et al., The Hispanic Population 2010, U.S. Census BUREAU (2010), available at www.census.gov/prod/cen2010/briefs/c2010br-04.pdf (last visited Mar. 3, 2012).

140. See The Henry J. Kaiser Family Found., Nebraska: Immigrants, STATE Health FactS, available at $\mathrm{http} / / \mathrm{www}$.statehealthfacts.org/profileind.jsp?cat=1 \&sub=157\&rgn=29 (last visited Mar. 3, 2012).

141. Id. 
It appears that it is not the size of the population that has prompted recent legislation, but rather the rate of growth. This issue, the question of whether foreign populations were arriving at rates higher than society believed could be assimilated into the United States has plagued the country since its inception some 300 million people ago. ${ }^{142}$ From colony specific concerns, through the treatment of imported African slaves followed by the Chinese exclusion laws, to the quota system of immigration, the United States has always been concerned with having too many immigrants, and these concerns have led to increased legislation in given time periods, and the result in Nebraska in 2011 is no different. ${ }^{143}$

Given both the immigrant population base and growth rate, it is not surprising that the immigrant community in Nebraska has significant economic importance, especially in certain sectors. Overall, immigrants provide approximately $7 \%$ of Nebraska's workforce ${ }^{144}$ (including an overwhelming $80 \%$ of the labor in meat processing positions). ${ }^{145}$ Perhaps the most important statistic yet to be mentioned, is the unemployment rate.

Nebraska, as of June 2011, has the second-lowest unemployment rate in the country ${ }^{146}$ and may be considered by some to already be at full

142. See, e.g., Ralph Frasca, "To Rescue the Germans out of Sauer's Hands:" Benjamin Franklin's German Language Printing Partnerships, 12 PA. MAG. OF HIST. \& BIOGRAPHY 329 (1997). In 1790, the estimated U.S. population was 3.9 million, as compared to 2011 's population of approximately 307 million people. See Population: 1790-1990, U.S. CENSUS BUREAU, tbl.4, http://www.census.gov/population/censusdata/table-4.pdf (last visited Mar. 3, 2012). In 1760 the population was estimated at approximately 1.6 million people. At that time, Benjamin Franklin wrote of the German immigrants, "[u]nless the stream of their importation can be tumed from this to other Colonies, they will soon so outnumber us, that all the advantages we have will not (in my Opinion) be able to preserve our language, and even our government will become precarious.". See Frasca, supra note 145, at 343 (citing Letter from Benjamin Franklin to Peter Collinson (May 9, 1753), in THE PAPERS OF BENJAMIN FRANKLIN 4:484-85 (New Haven 1959)).

143. Daniel Kanstroom, Deportation Nation 91-107, 219-20 (Harvard Univ. Press 2007).

144. See Selected Characteristics of Native and Foreign-Born Population 2009, U.S. CENSUS BUREAU, http://factfinder.census.gov/servlet/STTable?_bm=y\&-context=st\&-qr_name=ACS_2009_ 1YR_G00_S0501\&-ds_name $=$ ACS_2009_1YR_G00_\&-CONTEXT =st\&-tree_id=307\&-redoLog= false \&-geo_id =04000US3 1 \&-format=\&-_lang=en (last visited Mar. 3, 2012).

145. See Christopher S. Decker et al., Nebraska's Immigrant Population Economic and Fiscal Impacts, 2008 UNIV. OF NEB. 1, available at http:/www.unomaha.edu/ollas/ econ\%20im\%20report/econimpact.pdf (last visited Mar. 3, 2012). Importantly, immigrants are also fiscally neutral (and actually slightly positive) in Nebraska meaning that they pay in less than what they receive in government-sponsored benefits, including education and health services. $I d$. at 22 .

146. See Regional and State Employment and Unemployment Summary May 2011, U.S. BUREAU OF LABOR STAT., available at http://www.bls.gov/news.release/laus.nr0.htm (last modified Jan. 24,2012 ) (last visited Mar. 3, 2012). Nebraska's unemployment rate of $4.1 \%$ trails only North Dakota. Id. 
employment. ${ }^{147}$ Nebraska is also dealing with a few other interesting demographic factors such as negative net migration from the state and a looming labor shortage within the next decade. ${ }^{148}$

Additionally, Fremont (and Dodge County of which Fremont represents over $70 \%$ of the total county population) is largely representative of the greater state regarding the above dynamics. ${ }^{149}$ For example, from 2000 to 2010 , the county's Hispanic or Latino population more than doubled, ${ }^{150}$ and the total Hispanic or Latino population of the county is now over $10 \%$ of the total county population. ${ }^{151}$ Furthermore, this growth occurred at a time when overall county growth, in terms of population, was just $1.5 \%{ }^{152}$

It is against this demographic backdrop that state legislation was introduced in Nebraska in 2011 to criminalize unauthorized immigrants' employment in the United States, their failure to register or carry an alien registration receipt card, and knowing or reckless harboring of unauthorized immigrants. ${ }^{153}$ There were also laws to encourage all Nebraska counties and communities to enter into $287(\mathrm{~g})$ and/or Secure Communities Programs, ${ }^{154}$ expand mandatory use of E-Verify, ${ }^{155}$ and repeal the grant of in-state tuition to unauthorized immigrants who meet the statutory requirements. ${ }^{156}$

147. See 15 U.S.C. $\S 1022 \mathrm{a} ; 15$ U.S.C. $\$ \S 3101$ et seq. (showing that while there is debate as to the best definition of full employment, Congress has defined it as four percent).

148. See Steve Carlson et al, Brain Drain in Nebraska: What do the Data Show, 63 BuS. IN NEB. 1, 1-3 (2008) (explaining that the looming labor shortage, while not Nebraska specific, could turn out to be especially problematic for states already at or near full employment).

149. See Fremont (city), Nebraska, U.S. CENSUS BUREAU, http:/quickfacts. census.gov/qfd/states/31/31053.html (last modified Jan. 31, 2012) (last visited Mar. 3, 2012).

150. Ennis et al., supra note 142, fig. 6.

151. See Profile of General Population and Housing Characteristics: 2010, U.S. CENSUS BUREAU, http://factfinder2.census.gov/faces/tableservices/jsf/pages/productview.xhtml?pid=DEC_10_DP DPDP1\&prodType=table (last visited Feb. 10, 2012).

152. See Dodge County, Nebraska, U.S. CENSUS BUREAU, http:/quickfacts.census.gov/ $\mathrm{qfd} / \mathrm{states} / 31 / 31053$.html (last modified Jan. 31, 2012) (last visited Mar. 18, 2012).

153. See Neb. L.B. 48 (relating to the adoption of the Illegal Immigration Enforcement Act); see also Paul Hammel, Nebraska Legislature - Debate on Illegal Immigrants is Shelved, OMAHA WORLD HERALD, Mar. 9, 2011, at 4B (noting this bill and all other immigration-related proposals failed to advance out of committee in order to spend a year conducting an interim study on the topic).

154. L.R. 28, 102d Leg., 1st Sess. (Neb. 2011).

155. See Neb. L.B. 569.

156. See L.B. 657, 102d Leg., 1st Sess. (Neb. 2011) (indefinitely postponed). Not all proposed immigration-related legislation was "anti-immigrant" however as a bill was proposed (although not 
While not currently an issue in Nebraska as L.B. 48 and the other immigration-related proposals failed to advance, the one-year moratorium imposed by the committee while studying the recently adopted "Utah Compromise" could see the bills reintroduced in 2013. Likewise in Fremont, although the ordinance was adopted, it has never been enforced. ${ }^{157}$ In the Fremont case, the Court's decision in Hazelton and Whiting likely may have a significant impact. ${ }^{158}$ Unless the Third Circuit was to reaffirm its original decision in its entirety which seems unlikely, it appears that at least the employment portion of the ordinance may survive legal challenge. ${ }^{159}$

If that were to happen, the political landscape of Nebraska regarding immigration-related ordinances would likely be that of a state where local and state-level immigration-related policies would continue to be pushedthis time without the argument regarding the potential constitutionality of the laws and therefore with the less credible threat of deterrent litigation. In terms of race relations and economic well-being, such a situation seems troubling, given recent community-relation issues. ${ }^{160}$ However, the Nebraska legislature's decision to postpone any decision on immigrationrelated laws in 2011 in order to study Utah's approach also suggests a willingness to consider a wider range of legislation, a range that may not be possible unless shifts are made in the preemption debate.

enacted) to restore prenatal care coverage for all impoverished women (including unauthorized immigrants) as that benefit had been cut through legislation passed in 2010. See L.B. 599, 102d Leg., 1st Sess. (Neb. 2010) (relating to the medical assistance program to amend Neb. Stat. $\$ \$ 4-110$ and 68901).

157. See Cindy Gonzalez, Fremont Suspends Immigration Ordinance Until Suits are Resolved, OMAHA WORLD HERALD, July 28, 2010, at 04B (stating that following the city's adoption of the ordinance by referendum, the city council voted to suspend enforcement due to the threat of injunction).

158. See Keller v. City of Fermont, Nos. 8:10-cv-0270-LSC-FG3, 4:10-cv-3140-LSC-FG3, 2011 WL 41902, at 1 (D. Neb. 2011). See also Fremont, Neb., Ordinance 5165.

159. See Fremont, Neb., Ordinance $5165 \$ 5(\mathrm{a})-(\mathrm{h})$.

160. See, e.g., Michael Kelly, Prejudice Spoils a Night of Triump, OMAHA WORLd HERALD, May 20, 2010, at 02B (discussing the high school state championship soccer match where students threw "green cards" on the field in reference to the opposition school's location in a primarily Hispanic neighborhood). In Fremont specifically, fears and prejudice has arisen from the use of Spanish at the YMCA or Walmart. Even long-time residents have felt the increased vitriol in the community with one long-time resident businessman having his store window broken out. See Leslie Reed, June 21 Vote on Ordinance-Fremont is Still Torn on Immigrants, OMAHA WORLD HERALD, June 10, 2010, at 01A; Paul Hammel, Fremont Officials are Concerned About Tensions-The City Council is Considering a Resolution to Discourage "Divisive Behavior" in Regard to the Immigration Ordinance, ОМАHA WORLD HERALD, September 10, 2010, at 3B (citing 65 allegations of race-related harassment since the special election enacting the ordinance). 


\section{PRoposing A BILATERAL RATCheT}

In this article, I propose a more expansive approach to preemption, with some critical limitations in regard to subfederal immigration regulation. While a neutral, expansive approach has the potential benefit of allowing localities the ability to implement laws along a broader range of the spectrum, it has the potential downside to be concurrently used as a vehicle to advance discriminatory laws such as those struck down in California and Texas dealing with the education of unauthorized immigrant children, ${ }^{161}$ or a broader crime/enforcement law of the type enacted recently in Arizona, Georgia, and Alabama. ${ }^{162}$

Commentary on this issue has increased rapidly and is quite diverse in its breadth. ${ }^{163}$ On one side of the debate, Professor Pham has argued for an exclusive federal role in the immigration debate on constitutional and plenary power grounds. ${ }^{164}$ This type of argument was successfully used in the Third Circuit's decision in Hazelton, and at least partially in Arizona, but the Court's recent decisions provide for some role for subfederal legislation. Prior to the 2011 decisions, this approach was perhaps favored because of its ability to maintain a semblance of a balance between proimmigrant and pro-enforcement legislation. ${ }^{165}$

161. See, e.g., League of United Latin Am. Citizens v. Wilson, 908 F. Supp. 755, 764 (C.D. Cal. 1995); Plyler v. Doe, 457 U.S. 189, 189 (1982). In both cases, the courts struck down laws that purported to restrict access to elementary and secondary education.

162. See supra notes $135-237$ and accompanying text.

163. See, e.g., Keith Aoki \& John Shuford, Welcome to Amerizona-Immigrants Out!: Assessing "Dystopian Dreams" and "Usable Futures" of Immigration Reform, and Considering Whether "Immigration Regionalism" is an Idea Whose Time has Come, 38 FORDHAM URB. L.J. 1 (2010) (proposing regional immigration laws akin to the Canadian system that would allow regions some autonomy in determining immigration issues); Gabriel J. Chin et al., A Legal Labyrinth: Issues Raised by Arizona Senate Bill 1070, 25 GEO. IMMIGR. L.J. 47 (2010) (analyzing preemption issues related to S.B. 1070); D. Fan, Post-Racial Proxies: Resurgent State and Local Anti-"Alien" Laws and Unity-Rebuilding Frames for Antidiscrimination Values, 32 CARDOZO L. REV. 905 (2011); Pratheepan Gulasekaram, Sub-national Immigration Regulation and the Pursuit of Cultural Cohesion, 77 U. CIN. L. REV. 1441 (2009); Michael A. Olivas, Immigration-Related State and Local Ordinances: Preemption, Prejudice, and the Proper Role for Enforcement, 2007 U. CHI. LEGAL. F. 27 (2007); Pham, supra note 33 (arguing that a constitutional mandate for uniform immigration laws results in the preemption of subfederal laws that would otherwise result in a morass of conflicting or overlapping laws); Rodriguez, supra note 39 (arguing for a functional role for states and localities in immigration-related legislation while refuting the notion of federal exclusivity in immigration); Peter J. Spiro, Learning to Live with Immigration Federalism, 29 CONN. L. REV. 1627 (1997); Rick Su, A Localist Reading of Local Immigration Regulations, 86 N.C. L. REV. 1619 (2008) (arguing states and localities should play some role in immigration regulation).

164. See Pham, supra note 33, at 1003.

165. See Id. 
Professor Rodríguez, on the other hand, has argued that a uniform framework is neither legally mandated nor necessarily beneficial in a climate where little to no legislative action is occurring at the federal level. ${ }^{166}$ Professor Rodríguez, while conceding that some oversight is needed to prevent states and localities from producing negative externalities, argues forcefully for a "functional account" whereby courts and lawmakers alike would interpret preemption claims more narrowly in order to provide "ongoing dialogue ... at the state and local levels."167 The problem with this approach is that, while neutral, the current tenor of the immigration debate and the dramatic skewing of the current law towards enforcement have the opportunity to make this a largely anti-immigrant mechanism.

The intense subfederal push to regulate immigration has continued with harsher laws being proposed on a near daily basis. Even so, Professor Rodriguez ultimately concluded that a normalization of immigration authority is needed that would allow for coexisting state and federal regulation on immigration through the use of a standard preemption doctrine. This has merit. ${ }^{168}$ To a large extent this Article, in principle, agrees with these arguments, which seem to go fairly beyond the scope of the holding in De Canas. ${ }^{169}$ The test Professor Rodriguez provides, dealing specifically with conflict preemption (though seemingly applicable by analogy to field preemption as well) is whether the subfederal laws "thwart" federal enforcement of immigration law, ${ }^{170}$ not whether there is "tension with federal objectives." immigration statutes being proposed, this Article proposes that the normalization, in order to act as an effective "normalization," must take its shape as a largely pro-immigrant (not necessarily pro-immigration) device to provide a level legislative field in which states and localities could act. ${ }^{172}$

166. See Rodriguez, supra note 39, at 571-72 (stating that uniform legislation is both "difficult to achieve, [and] it is also often counterproductive").

167. Id. at 596. In policing some of the negative externalities, Professor Rodriguez would also rely on other statutory and constitutional protections such as the First and Fourteenth Amendments and other landlord and tenant laws. Id. at 572.

168. See Rodríguez, supra note 39, at 610-11, 620, 622-23 (establishing a framework for the implementation of the normalization process which would require a shift in federal preemption doctrine as well as the use of anti-preemption presumptions).

169. Id. at 624 (arguing that landlord regulations are only tangentially related to removal or ICE and should therefore not be considered to be regulating immigration).

170. Id. at 626.

171. Id. at $610-11$.

172. Id. at 617 . 
Implementation of this type of preemption doctrine has the benefit of overall federal neutrality. The limit on pro-enforcement, subfederal immigration legislation would remain static while pro-immigrant legislation would be permitted to allow states to craft, within established limits, programs tailored for their specific regions. The neutrality envisioned would allow states to enact more types of immigration-related legislation which would likely include the judicially-accepted portions of S.B. 1070 type laws as well sanctuary and potentially even employment-related type laws. This type of approach, applied properly, could reach that portion of Utah's law which seeks to grant state-issued work permits while also limiting the more deleterious provisions.

As it stands, immigration preemption analysis is at a crossroads. Without a relaxation or reinterpretation of the preemption doctrine, the current system allows states little room for leniency in terms of allowing the presence of unauthorized immigrants, while allowing states great latitude to increase enforcement measures either in conjunction with the federal government or through unilateral "licensing" laws. Given that De Canas appears to have been revived and that Congress has expressly provided for anti-immigrant, subfederal immigration regulation, this Article asks whether there might not be room for this third way.

Accepting Whiting as our current state of immigration preemption law (and knowing that an upcoming decision in Arizona could fundamentally alter the De Canas and/or Hines approaches), it would appear that a counterbalance in favor of subfederal, pro-immigrant legislation should be allowed so that states could enact legislation that would also satisfy preemption and other federalism arguments. If controversial measures such as the revocation of a business license, mandatory implementation of $\mathrm{E}$ Verify and potentially even occupancy licenses are allowed, why not also allow (or encourage) subfederal driver's license laws, ${ }^{173}$ locally-issued work permits, Mini-Dream Act laws, or sanctuary laws?

So the question is then whether there exists a legitimate distinction that can be made between laws that are acceptable encroachments on the traditional federal immigration framework while not going so far as to open the floodgates and allow clearly discriminatory laws such as Proposition 187 that ignite a race to the bottom among the states? ${ }^{174}$ I believe the

173. See Real ID Act, Pub. L. No.109-13, 19 Stat. 311, § 202(a)(1) (2005) (codified at 49 U.S.C. $\S 30301$ ) (explaining that while not per se prohibited by federal law, the Real ID Act encourages states to make their licenses more secure. Failure to provide such security could render the license invalid for boarding airplanes or gaining access to other federal facilities).

174. See Rodríguez, supra note 39, at 640 n.303 (citing Peter H. Schuck, Some Federal StateDevelopments in Immigration Law, 58 N.Y.U. ANN. SURV. AM. L. 387, 389 (2002)) (stating that no 
answer is yes. The floor for pro-enforcement, subfederal legislation has been set primarily through the interpretation of the Savings Clause. However, for pro-immigrant, subfederal legislation, the question is more difficult as there is no corollary Savings Clause to provide states and localities the legislative flexibility required.

Clearly the Holy Grail for proponents of increased immigration would be state-authorized presence and employment. Under the current legal framework (which until very recently appeared unlikely to undergo dramatic structural change in the near future), state-issued visas are not a possibility as a direct regulation of immigration. While another alternative, state-authorized employment work permits, has been suggested before without success, ${ }^{175}$ this Article would include state-authorized work permits under the rubric of acceptable state action in allowing subfederal, proimmigrant legislation.

The key to a successful presence in the United States for unauthorized immigrants is access. Immigrants need access to housing, transportation, employment, education, and public services (in the sense of access to police protection, fire departments, and the legal system). Denying access to any of these categories increases the likelihood that the unauthorized immigrants will be preyed upon and subjected to victimization. Of these five categories, only education has been decided in favor of immigrants, ${ }^{176}$ while subfederal laws have consistently attacked the remaining four. ${ }^{177}$ In order to allow for counterbalancing laws, somehow states would need to be allowed to legislate with fairly wide discretion in this realm subject to the constraints noted above.

One potential legal mechanism to achieve such a result is to eliminate the presumption of preemption and encourage an immigrant-neutral dual state-federal system. ${ }^{178}$ As recognized, such an approach could allow for unacceptably discriminatory legislation if no limits were established. ${ }^{179}$ The mechanism proposed, perhaps easier to implement in theory and of

ascertainable race to the bottom occurred in 1996 when states were allowed to prohibit public benefits under IIRIRA § 553).

175. See Bill Ong Hing, NAFTA, Globalization and Mexican Migrants, 5 J.L. ECON. \& POL'Y $87,166-67$ (2009) (noting that lowa in 2010 established a commission to improve the state's economy, and that among its recommendations was to "Designate lowa an 'immigrant enterprise zone' and seek a federal exemption from immigration quotas").

176. See, e.g., Plyler, 457 U.S. at 189.

177. Even though no laws have attempted to deny police or fire protection to unauthorized immigrants, laws that would require any law enforcement personnel to determine the immigration status of anyone with whom they have lawfully come into contact clearly chill the exercise of that right.

178. See supra note 174 and accompanying text.

179. See supra note 178 . 
limited utility, would be to borrow the concept of express approval from the Savings Clause to apply it to pro-immigrant legislation - recognizing that for it to work as expected, new granting language would be required in the INA. ${ }^{180}$ Other than the ineluctable problem of enacting such language, there is arguably judicial support for the approach as it was the approach the Court implicitly followed in Martinez. ${ }^{181}$

The Court, using the canon of plain language, held the statute prohibited a very specific type of benefit - one granted on residence only, and therefore, all other conduct regarding postsecondary benefits was saved. ${ }^{182}$ Likewise, with state benefits, states retain the right to grant public benefits to unauthorized immigrants, and cases have followed using plain language to validate subsequent state laws granting benefits even when the statutes did not reference $\S 411$ of the Personal Responsibility and Work Opportunity Reconciliation Act of 1996 (PROWRA). ${ }^{183}$ These are two well-known, though limited, examples where the judiciary has inferred a reverse savings clause.

Referring back to the other categories of access needed for immigrants, there is also express legislative language that technically allows states to continue to grant driver's licenses (though such licenses do not allow for interstate plane travel) ${ }^{184}$ as well harboring statutes that are arguably not so broad as to include the rental or sale of housing to unauthorized immigrants (though they are broad enough to include sanctuary policies). ${ }^{185}$ These statutes do not, however, have anywhere near the same strength as the Savings Clause in that they require almost hypertechnical readings in order to contemplate pro-immigrant benefits. ${ }^{186}$ Furthermore, in both cases, there are serious consequences for failure to

180. See supra notes $179-80$.

181. See Martinez, 241 P.3d at 855.

182. See Id. at 855,865 .

183. Personal Responsibility and Work Opportunity Reconciliation Act of 1996, 42 U.S.C. $\S$ 1305 (2006).

184. See Real ID Act, Pub. L. No.109-13, 19 Stat. 311, § 202(a)(1) (2005) (codified at 49 U.S.C. $\$ 30301$ ). There has been much excellent commentary on the repurposing of driver's licenses to act as a national identification card. See, e.g., Kevin R. Johnson, Driver's Licenses and Undocumented Immigrants: The Future of Civil Rights Law?, 5 NEv. L.J. 213, 221 (2004).

185. See 8 U.S.C. $\S 1324$ (providing that harboring requires knowing or reckless disregard of unlawful presence). See, e.g., Merkt, 794 F.2d at 950 (upholding harboring conviction of defendants who claimed they were providing "sanctuary" based on religious beliefs). The problem with the renting or sale of housing could arise with the now-ubiquitous practice of utilizing social security numbers when performing credit checks. Improper or no social security number, coupled with other factors, may rise to the level of "reckless disregard."

186. See supra notes $202-03$ and accompanying text. 
comply with the desired federal scheme. ${ }^{187}$ Most importantly, no reverse Savings Clause interpretation is available regarding pro-immigrant employment provisions, the most important right in the eyes of most unauthorized immigrants, and the area where states are most critically handicapped. ${ }^{188}$ Quite simply, as was the purpose of Congress, there is no reading, plain language or otherwise, that would allow states to authorize work permits without a significant modification of current law. ${ }^{189}$

The purpose of this Article, then, is to advocate an approach between the competing theories of concurrent state/federal regulation of immigration and the current framework which provides states and localities little freedom to implement pro-immigrant legislation. ${ }^{190}$ This Article posits the easiest way to do so is by granting states the express ability to regulate the five areas of access above with regard to pro-immigrant legislation. ${ }^{191}$ The proposal is slightly unpalatable in the sense that it requires Congressional action (or at least acquiescence as foreseen by Utah in the employment context), but it is difficult to conceive of any other course that would allow states the ability to regulate immigration-related matters locally other than in solely pro-enforcement ways. ${ }^{192}$

187. See Nicholas Confessore, After a Rough Start, Spitzer Rethinks His Ways, N.Y. TIMES, Nov. 27, 2007, at Al (illustrating that New York's citizenry was quick to object to proposed driver's licenses for all upon learning that their driver's licenses would no longer be valid for airline travel). Anecdotally, the federal government has also prosecuted landlords for harboring via renting an apartment or home. See, e.g., Brandon Ortiz, Immigration Case Puts Focus on Landlords, Houston Chronicle, May 25, 2008, at A3. Cf. Recalde v. BAE Cleaners, Inc., 862 N.Y.S.2d 781 (N.Y. Sup. Ct. 2008). In addition, federal, state, and local fair housing laws likely prohibit any discrimination based on national origin, and therefore questions of based on alienage may result in violations. See, e.g., Sophie Marie Alcorn, Landlords Beware, You may be Renting Your own Room . . in Jail: Landlords Should not be Prosecuted for Harboring Aliens, 7 WASH. U. GLOBAL STUD. L. REV. 289, 304 (2008).

188. See 8 U.S.C. $\S 1324$ a (showing that INA $\S 274 a$, implemented through IRCA, is broad, strict, and comprehensive in its prohibition on employing unauthorized immigrants, though it does have two notable exceptions: the first is for "casual employment by individuals who provide domestic service in a private home that is sporadic, irregular or intermittent" and independent contractors); 8 U.S.C. $\S 1324 \mathrm{a}(\mathrm{a})(4)$ (prohibiting the hiring of independent contractors "knowing that the alien is an unauthorized alien ....").

189. See supra note 206 and accompanying text.

190. See infra note 210 and accompanying text.

191. Education and rights to access deserve additional explanation. The right to education was decided primarily on equal protection grounds and therefore an expansion of states' abilities to legislate in this area would not permit them to override constitutional protections. See Plyler, 457 U.S. at 210 14. As for access to public services, equal protection again would prevent states from denying such services, though Secure Communities-type initiatives or the majority of lawful contact immigration determinations would (with the possible exception being those that could occur in a school setting).

192. See supra notes $179-81$. 
How then, would such an approach deal with a law such as S.B. 1070, Nebraska L.B. 48, Fremont Ordinance 5165, or any of their copycats? As to S.B. 1070, which has been litigated, the provision that withstood challenge would continue to be valid. As to those provisions that were struck down on preemption grounds, all or nearly all would continue to be enjoined under this new scheme. Likewise, Fremont's Ordinance would face the same result as is likely for Hazleton-an injunction on the portion of the ordinance relating to occupancy permits as impermissibly regulating immigration, but an allowance of the employment provisions. One can see then that this approach does not represent such a dramatic shift from today's preemption scheme or from the holding in Hines that strongly cautioned against discriminatory treatment of authorized immigrants to leave them "free from the possibility of inquisitorial practices and police surveillance" 193 as one might initially conceive.

\section{CONCLUSION}

It is not often that people engaged in a passionate debate find themselves at such a clearly marked, defining moment. With the turmoil in immigration-related preemption law and subfederal attempts to enter the field, we currently find ourselves at such a crossroad. While prorestrictionists may very well abhor the type of proposal this Article suggests, they should pause to reconsider. For one, in those states desirous of fewer immigrants, there may be few mechanisms as effective in obtaining voluntary immigration as a neighboring jurisdiction that protected an immigrant's access to the categories above. ${ }^{194}$ Second, the proposal, while pro-immigrant in and of itself, is immigrant-neutral in the overriding legal framework and only seeks a level playing field in which states are permitted to act. Finally, the proposal contemplates the past trends regarding depressed economies and anti-immigrant legislation as well as future trends of declining birth rates and potential labor shortages in a way that the stream of current legislation does not.

In the end it appears that the overriding federal supremacy and regulation of immigration laws is drawing to a close as states and localities are expressly being granted the right to regulate. We should take care to assure that the regulation is not one-sided, and we should recognize that a unilateral ratchet towards enforcement is unlikely to diffuse the animosity of the current debate. Providing states and localities a voice and a role, both pro-enforcement and pro-immigrant, in shaping the current legislation

193. Hines, 312 U.S. at 74.

194. Professor Spiro, over a decade ago, called for a preemption framework to allow states to be "laboratories towards best practices." See Spiro, supra note 165, at 1636 n.34. 
may be the best way for the debate to evolve when faced with the very real possibility that no federal, comprehensive efforts will be forthcoming in the near future. 



\title{
ESTUDIO JUDICIAL DE LA LEY DE SEGURIDAD NACIONAL E INMIGRACIÓN: ENFOQUE CONVERGENTE SOBRE EL PODER DEL ESTADO Y LOS DERECHOS INDIVIDUALES
}

\author{
J. Hafetz*
}

I. LA INFLUENCIA DE LA LEY DE INMIGRACIÓN SOBRE LA POLÍTICA DE SEGURIDAD NACIONAL ...............................................744

A. Encuadre del debate: cambiar derechos por seguridad .........744

B. El desarrollo de dos niveles de estructuras de adjudicación.. 747

C. Restringir el acceso a las cortes y reexaminar el alcance...... 751

D. Seguridad como poder para otros objetivos ...........................756

II. EL FRACASO DE NO CERRAR GUANTÁNAMO.....................................759

A. Guantánamo y el trato diferencial a los no ciudadanos ..........760

B. El fracaso de reubicar a los detenidos en los Estados

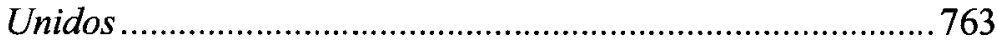

C. La doctrina de poderes plenos y litigios de habeas en

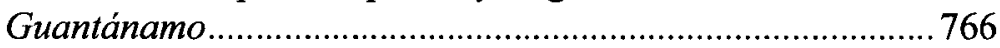

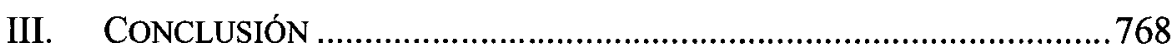

A partir del ataque terrorista del 11 de septiembre del 2001, la ley de seguridad nacional se ha expandido como un campo de estudio. ${ }^{1}$ En la pasada década se ha visto un crecimiento exponencial de investigaciones, ofertas de cursos, congresos y programas orientados a las políticas de seguridad nacional en Estados Unidos. ${ }^{2}$ A la vez, temas candentes tales como, la detención, el trato, y los juicios a sospechosos de terrorismo han llamado la atención de muchos académicos a nivel nacional y del espectro internacional. Gran parte de la literatura en esta área esta orientada hacia la naturaleza "excepcional" de las políticas implementadas después del 11 de septiembre, lo que explica por qué y cómo esas políticas representan un

* Profesor Asociado de la Facultad de Derecho de la Universidad Seton Hall. Este artículo se basa en mis observaciones del Intecambio LatCrit Sur-Norte del 2011, en Santo Domingo, República Dominicana (13 de mayo de 2011).

1. Michael R. Gordon, A DAY OF TERROR: AN ASSESSMENT; When an Open Society Is Wielded as a Weapon Against Itself [UN DIA DE TERROR: UNA EVALUACIÓN; Cuando una Sociedad Abierta se Ejerce Como un Arma Contra Si Misma], N.Y. TiMEs (12 de septiembre del 2001), disponible en http:/www.nytimes.com/2001/09/12/us/day-terror-assessment-when-open-societywielded-weapon-against-itself.html?ref=sept112001 (última visita el 30 de marzo del 2012).

2. Ver, por ejemplo, Joseph Margulies \& Hope Metcalf, Terrorizing Academia [Aterrorizando Academia], 60 J. LEGAL. ED. 433, 440-41 (2011) (discutiendo la tendencia). 
significante, si no radical, rompimiento con normas y comportamientos anteriores. $^{3}$

Algunos, sin embargo, han cuestionado la separación de la ley de seguridad nacional como disciplina. Aziz Huq, por ejemplo, ha debatido en contra del "excepcionalismo de la seguridad nacional," explicando que las respuestas judiciales a las emergencias de seguridad nacional parecen estar más estrechamente asociadas a las tendencias transubstanciales de la ley pública y judicial y las emergencias que no son de seguridad. ${ }^{4}$ En una línea parecida, Judith Resnik ha enfatizado la continuidad de la jurisprudencia entre la "guerra en terrorismo" después del 11/09 en los Estados Unidos y el trato a los delincuentes acusados, los presos condenados y los inmigrantes, ${ }^{5}$ mientras que James Forman ha enfatizado los paralelos existentes entre el trato a los "combatientes enemigos" en Guantánamo ${ }^{6}$ y los indigentes acusados en los Estados Unidos. ${ }^{7}$

Los académicos también han debatido como el creciente énfasis en la seguridad nacional ha impactado las leyes y políticas de inmigración después del 11/09. Jennifer Chacon, por ejemplo, describe cómo la retórica de la seguridad nacional ha distorsionado el debate sobre el control de la inmigración y el crimen. ${ }^{8}$ Kevin R. Johnson y Bernard Trujillo han explicado cómo la preocupación por la seguridad nacional ha llegado a dominar la discusión sobre una reforma comprensiva de inmigración. ${ }^{9}$

3. Ver id. at 440 ("Una revisión de la literatura legal post-9/11 revela un estudio academic obsesionado con lo excepcional."); Judith Resnik, Detention, The War on Terror, and the Federal Courts [Detención, La Guerra Contra el Terror, y los Tribunales Federales], 110 CoLUM. L. REv. 579, 595-96 (2010) (describiendo la expansion de la cobertura de los casos de la "guerra contra el terrorismo" en un libro de casos líder de los tribunals federales).

4. Aziz Z. Huq, Against National Security Exceptionalism [Contra el Excepcionalismo de la Seguridad Nacional], 2009 SUP. CT. REV. 225, 227 (2009).

5. Resnik, supra nota 3, en 577-78.

6. James Forman, Jr., Exporting Harshness: How the War on Crime Helped Make the War on Terror Possible [Exportación de Severidad: Cómo la Guerra contra el Crimen ayudó a que la Guerra contra el Terror sea Posible], 33 N.Y.U. REV. Of LAW \& SOC. Change 331 (2009). Para una perspectiva similar, basada en sus años de representación de acusados capitales en Louisiana, ver Denny LeBoeuf, From the Big Easy to the Big Lie [Desde lo Gran Fácil a la Gran Mentira], in THE Guantánamo LaWYers: INSIDE a PRISON OUTSIDE THE LAW (Mark Denbeaux \& Jonathan Hafetz, eds.) (2010).

7. Id.

8. Jennifer M. Chacon, Unsecured Borders: Immigration Restrictions, Crime Control and National Security [Fronteras Inseguras: Restricciones de Immigración, Control del Crimen y Seguridad Nacional], 39 CONN. L. REV. 1827, 1830 (2007).

9. Kevin R. Johnson \& Bernard Trujillo, Immigration Reform, National Security afier September 11, and the Future of North American Immigration [Reforma de Inmigración, Seguridad 
Como estos académicos debaten, el enfoque miope en el terrorismo no sólo ha llevado a un incremento inexorable en las medidas de detención y deportación; sino que incluso ha creado un vacío entre la retórica de seguridad y la realidad de protección limitada para los inmigrantes sin incrementar la seguridad. ${ }^{10}$

Este artículo discute temas similares pero desde una perspectiva diferente. El artículo examina cómo conceptos que originalmente se desarrollaron en el contexto de la ley de inmigración han resurgido después del 11/09 en la jurisprudencia de seguridad nacional y han ayudado a configurar al acercamiento de los Estados Unidos con respecto a la detención y el trato de los sospechosos de terrorismo. A primera vista, la constelación de temas de seguridad nacional después del 11/09 pueden parecer distintos de la ley de inmigración. Juicios por comisiones militares, encarcelamiento de combatientes enemigos, y el asesinato selectivo de sospechosos de terrorismo, por ejemplo, pueden parecer algo distinto a la detención y la deportación de aquellas personas que no son ciudadanos bajo la ley de inmigración. Los primeros están sujetos a la toma de decisiones y jurisdicción militar, no civil, involucran al poder ejecutivo en tiempo de guerra y están justificados bajo los principios de la ley de la guerra entre otras diferencias.

Sin embargo, como se explica en este artículo, existen importantes semejanzas. Estos vínculos ilustran cómo los conceptos de derechos y participación en la política influyen la reacción de los Estados Unidos sobre el trato de los sospechosos de terrorismo. Ellos también proveen otra perspectiva sobre algunas grandes fuerzas que configuran la reacción de los Estados Unidos a preocupaciones de seguridad nacional.

La primera parte describirá varias áreas de superposición entre la inmigración y la ley de seguridad nacional. Éstas incluyen el uso de discursos que enfrentan los derechos de otros (si se definen como inmigrantes o sospechosos de terrorismo) contra la seguridad pública; el desarrollo de un sistema adjudicador a dos niveles legitimados por la clasificación del gobierno de la naturaleza de la privación de libertad; restricciones en el acceso a los tribunales y limitaciones en la revisión judicial; y el uso de la seguridad como poder para otros programas. La segunda parte examina cómo el intento fallido del presidente Obama para cerrar Guantánamo subraya cómo la política de inmigración estadounidense y la jurisprudencia siguen influyendo y moldeando el acercamiento de los

Nacional Después del 11 de Septiembre, y el Futuro de la Inmigración de América del Norte], 91 MNN. L. REV. 1369, 1373 (2007).

10. Ver generalmente Chacon, supra nota 8. Ver también Johnson \& Trujillo, supra nota 9. 
Estados Unidos respecto a la detención y el enjuiciamiento de los sospechosos de terrorismo.

\section{LA INFLUENCIA DE LA LEY DE INMIGRACIÓN SOBRE LA POLÍTICA DE SEGURIDAD NACIONAL}

Después del 11/09, la política antiterrorista de los Estados Unidos tomo un nuevo rumbo, lejos de un paradigma de aplicación de la ley y hacia lo militar, una ley basada en el modelo de la guerra. ${ }^{11}$ Importante para este acercamiento fueron la detención de los sospechosos de terrorismo como "combatientes enemigos," el uso de comisiones militares para juzgar delitos de terrorismo, y, por lo menos inicialmente, el uso de duros métodos de interrogación que rayaban en, y en algunos casos llegaron a, la tortura. ${ }^{12}$ En la aplicación de estas políticas en Guantánamo y otras prisiones ubicadas fuera del país, los Estados Unidos intentó evitar cualquier protección legal bajo la ley nacional o derecho internacional y negar el acceso de los prisioneros a los tribunales. ${ }^{13}$ Aunque estas políticas marcaron una ruptura con el pasado - parte de lo que la administración Bush denominó un "nuevo tipo de guerra"- tienen importantes similitudes en cuanto al enfoque de los Estados Unidos respecto a la inmigración y el trato a los no ciudadanos en general. ${ }^{14}$

\section{A. Encuadre del debate: cambiar derechos por seguridad}

La ley de inmigración se basa fundamentalmente en una dicotomía entre los ciudadanos y los no ciudadanos, ya que regula el derecho de los no ciudadanos de entrar y permanecer en el país. ${ }^{15}$ La ley de inmigración sin embargo ha servido durante mucho tiempo como un vehículo para expresiones de sentimientos xenofóbicos más amplios que trascienden las preguntas sobre la regulación de las fronteras. Durante la segunda mitad del siglo diecinueve, por ejemplo, las actitudes racistas hacia los chinos contribuyeron a poner en marcha y dar paso franco a la Ley de Exclusión

11. Ver, por ejemplo, Laurie R. Blank, Principles of Counter-Terrorism Law [Principios de la Ley Contra el Terrorismo], 25 EMORY INT'L L. REV. 771, 772 (2011) (destacando el cambio de paradigma en su reseña del libro de Jimmy Gurulé y Geoffrey S. Corn con el mismo título).

12. Para una visión general sobre estos desarrollos, ver JONATHAN HAFETZ, HABEAS CORPUS AFTER 9/11: CONFRONTING AMERICA'S NEW GLOBAL DETENTION SYSTEM [HABEAS CORPUS DESPUÉS DEL 9/11: CONFrontando el NuEvo Sistema Global de DeTENCIÓn AMERICANo] (2011).

13. Id.

14. Ver generalmente Johnson \& Trujillo, supra nota 9.

15. Ver Gabriel J. Chin, Segregation's Last Stronghold: Race Discrimination and the Constitutional Law of Immigration [Último Bastión de la Segregación: Discriminación Racial y la Ley Constitucional de la Inmigración], 46 U.C.L.A. L. REV. 1, 12-15 (1998). 
China y esto asentó las bases para siguientes leyes de motivación racial. ${ }^{16}$ La corte suprema unió al poder del gobierno en sus esfuerzos para contener la "gran multitud de ciudadanos chinos" buscando entrar a los Estados Unidos, y así dar todo el poder al gobierno para garantizar la seguridad y estabilidad del país. ${ }^{17}$ Después de las redadas de Palmer de 1919-1920, los extranjeros se representaban como un peligro para la seguridad pública con el fin de justificar duras restricciones de inmigración y las políticas de deportación. ${ }^{18}$ Esta tendencia se mantuvo durante toda la guerra fría, y fue manifestada, por ejemplo, en la exclusión de los no ciudadanos basada en puntos de vistas políticos considerados contrarios a la seguridad del país. ${ }^{19}$ La fusión del control de la inmigración y la seguridad nacional ha aumentado de manera sostenida desde mediados de los años noventas, especialmente después del 11/09, concentrada en la lucha contra el terrorismo global. ${ }^{20}$

En la ley de inmigración, el debate está típicamente enmarcado como un concurso de resultado nulo entre la seguridad por un lado, y los derechos y el bienestar de los inmigrantes, por el otro. Mientras más preocupación por el terrorismo global impregna ese debate, más aguda se vuelve ese argumento. Los temores sobre el terrorismo fuerzan tanto la situación, que los funcionarios públicos, los legisladores, y los comentaristas crean y sostienen una narrativa en la cual la seguridad del país depende de las restricciones de los derechos de los no ciudadanos dentro y fuera de las fronteras de América.

Una narrativa similar se ha arraigado en el contexto de la ley de seguridad nacional después del 11/09. Las medidas de "guerra contra el terrorismo," como la detención de "combatientes enemigos," el uso de comisiones militares y la dependencia en duros métodos de interrogación para extraer información de inteligencia, se basan en la suposición de que a los sospechosos de terrorismo- 0 por lo menos a los sospechosos de terrorismo que no son ciudadanos-no se les debe conceder la misma protección que a otros individuos. A un nivel, estas medidas deben verse desde la perspectiva de "un estado excepción:" aquella en que las

16. Id.

17. Chacon, supra nota 8, en 1833-34.

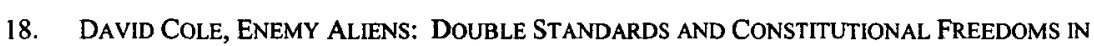
tHE WAR ON TERrorism [EXTRANJERos ENEMIGOS: Doble CRIERTIO Y Libertades CONSTituCiónales en la GuerRa Contra el TERrorismo] 85-153 (The New Press 2003).

19. Ver Timothy Zick, Territoriality and the First Amendment: Free Speech at-and Beyond -Our Borders [Territorialidad y la Primera Enmienda: Libertad de Expresión en-y Más Allá de-Nuestras Fronteras], 85 Notre DAME L. Rev. 1543, 1552-54 (2010) (sobre las provisiones ideológicas de exclusión de la Ley McCarren-Walter).

20. Id. en 1834; COLE, supra nota 18. 
amenazas mortales a la política crean presiones para apartarse o buscar exenciones de las normas ordinarias. ${ }^{21}$ Después del $11 / 9$, esta teoría justificó excepciones a reglas establecidas, prácticas, y protecciones a debidos procesos encarnados a través de la aprobación de enfoques militares basados en la política de la ley de la guerra contra el terrorismo. ${ }^{22}$ Sin embargo varias de las políticas de la "guerra contra el terror"especialmente aquellas que le permiten al gobierno detener a sospechosos de terrorismo sin un juicio penal federal - han afectado exclusivamente a los no ciudadanos, aunque los ciudadanos planteen y muchas veces hayan planteado, una amenaza terrorista equivalente a los no ciudadanos. ${ }^{23}$ Además, estas políticas están justificadas no como sacrificios compartidos o temporales para enfrentar un peligro inminente, sino como limitaciones necesarias y potencialmente permanentes justificadas por la condición de inferioridad legal de los no ciudadanos, que no comparten los mismos derechos que los ciudadanos estadounidenses. ${ }^{24}$

En resumen, el marco utilizado en intentos previos para restringir los derechos de los inmigrantes al oponer esos derechos contra la seguridad del país ha resurgido continuamente después del 11/09 en la construcción de la narrativa de seguridad nacional que depende de la limitación de derechos a los no ciudadanos. La diferencia más significativa entre las narrativas de inmigración y la "guerra contra el terrorismo" es en última instancia una del ámbito de aplicación: mientras que la ley de inmigración se concentra en los Estados Unidos, las medidas de contraterrorismo se concentran tanto en el país como en el exterior, proporcionando las bases para restringir los derechos de los no ciudadanos no sólo dentro de los Estados Unidos sino también en centros de detención ubicados en el extranjero como es el caso de Guantánamo.

21. Ver Mark Danner, After September 11: Our State of Exception [Después del 11 de septiembre: Nuestro Estado de Excepción], NEW YORK REVIEW OF BOOKS (13 de octubre del 2011), disponible en http:/www.nybooks.com/articles/archives/2011/oct/13/after-september-11-our-stateexception (última visita el 30 de marzo del 2012).

22. Ver Kim Lane Scheppele, Law in Time of Emergency: States of Exception and the Temptations of $9 / 11$ [Derecho en Tiempo de Emergencia: Estado de Excepción y las Tentaciones del 9/1/], 6 U. PA. J. ConsT. L. 1001, 1003 (2004) (sobre la tentación de ver la respuesta de los Estados Unidos a los ataques terroristas del 9/11 a través del marco del estado de excepción).

23. Ver id. en 1028, 1048 (señalando, respectivamente, la ciudadanía Estadounidense de John Walker Lindh y Hamdi Yaser).

24. Ver, por ejemplo, Robert M. Chesney, Who May Be Held? Military Detention Through the Habeas Lens [A Quién se le Puede Detener? Detención Militar través del lente de Habeas], 52 B.C. L. REV. 769, 812 (2011) (discutiendo la distinción trazada entre los derechos de los ciudadanos y no ciudadanos por el Tribunal de Distrito de EE.UU. para el Distrito de Carolina del Sur en Al-Marri v. Hanft, 378 F. Supp. 2d 673 (D.S.C. 2005)). 


\section{B. El desarrollo de dos niveles de estructuras de adjudicación}

Otra forma importante en casos de la "guerra contra el terror" es el eco de la ley de inmigración en su creación de formas alternativas de adjudicación que ofrecen de manera significativa mucho menos protección legal que el proceso criminal cuando se priva a un individuo de su libertad. ${ }^{25}$ Hace más de un siglo, la corte suprema consideraba que la deportación no era "castigo por un delito" sino "un método para exigir el regreso a su país de un extranjero que no había cumplido con . . . las condiciones" para continuar su residencia en los Estados Unidos. ${ }^{26}$ Definir la deportación como algo civil, en vez de un delito penal, ayudó a justificar la negación a los inmigrantes que enfrentan ser deportados del país las mismas protecciones constitucionales que ofrecen a aquellos que se enfrentan a ser condenados por cometer delitos criminales, incluyendo el derecho a un juicio por un jurado y la prohibición de leyes ex post facto. ${ }^{27}$ La detención de los no ciudadanos ha sido caracterizada como parte de la deportación, lo cual evita desencadenar todo el conjunto de protecciones constitucionales, en la medida en que la privación de libertad está vinculada al proceso de deportación de inmigración. ${ }^{28}$ Esta visión de la deportación como civil en vez de criminal ha persistido por más de un siglo, a pesar del reconocimiento de la corte suprema de que lo efectos de la deportación pueden ser extremadamente duros, similares al destierro o al exilio. ${ }^{29}$ Así, mientras que la corte ha requerido que los procedimientos de deportación satisfagan los procesos de un juicio justo, estos procedimientos son

25. Ver Jesselyn A. Radack, You Say Defendant, I Say Combatant: Opportunistic Treatment of Terrorism Suspects Held in the United States and the Need for Due Process [Usted Dice Acusado, Yo Digo Combatiente: Tratamiento Oportunista de Sospechosos de Terrorismo Detenidos en los Estados Unidos y la Necesidad del Debido Proceso], 29 N.Y.U. REV. L. \& SoC. Change 525, 535 (2005) (describiendo la conclusión del Grupo de Trabajo sobre el Tratamiento de Combatientes Enemigos de la ABA que "son detenidos sin cargos y son ciudadanos estadounidenses tienen menos derechos y protecciones que los que han sido acusados de delitos graves").

26. Fong Yue Tong v. United States, 149 U.S. 698, 730 (1893).

27. Ver Daniel Kanstroom, Deportation, Social Control, and Punishment: Some Thoughts about Why Hard Laws Make Bad Cases [Deportación, el Control Sacial y el Castigo: Algunas Reflexiones Acerca de Por Qué Leyes Duras Hacen Casos Malos], 113 HARV. L. REV. 1889, 1899-1906 (2000).

28. Ver Whitney Chelgren, Preventive Detention Distorted: Why It Is Unconstitutional to Detain Immigrants Without Procedural Protections [Detención Preventiva Distorsionada: ¿Por Qué es Inconstitucional Detener a Inmigrantes sin las Garantias Procesales], 44 LoY. L.A. L. REV. 1477, 1513-14 (2011) (explicando cómo el poder de detener no inherentes a la facultad de remover o excluir).

29. Padilla v. Kentucky, 130 S. Ct. 1473, 1481-82 (2010); Galvan v. Press, 347 U.S. 522,530 (1952) (observando que deportación puede "privar a un hombre de todo lo que hace que la vida valga la pena") (cuotando $\mathrm{Ng}$ Fung Ho v. White, 259 U.S. 276, 284 (1922) (comillas internas omitidas)); Galvan, 347 U.S. en 530 (comparando la deportación al destierro o el exilio). 
considerablemente menos fuertes que los que le ofrecen a aquellos acusados que enfrentan enjuiciamiento penal. ${ }^{30}$ Por otra parte, la caracterización de la inmigración como algo civil- $\mathrm{y}$ por lo tanto fuera de las protecciones del sistema de justicia penal-ha contribuido a mantener la autoridad del gobierno de manera amplia y en gran medida su inapelable autoridad para deportar extranjeros del país en virtud de la llamada doctrina de los poderes plenos. $^{31}$

La detención de militares y el enjuiciamiento a los sospechosos de terrorismo después del 11/09 ha seguido un patrón similar, con el desarrollo de mecanismos de adjudicación alternativos diseñados para proporcionar menos protecciones que el sistema de justicia penal. ${ }^{32}$ El gobierno de Estados Unidos a impuesto su autoridad para detener a individuos de manera indefinida sin cargos basado en su clasificación de "combatientes enemigos. ${ }^{, 33} \mathrm{La}$ corte suprema en general ratificó este enfoque en Hamdi v. Rumsfeld, ${ }^{34}$ considerando que la detención de estos combatientes enemigos, para evitar su regreso al campo de batalla es un hecho aceptado y fundamental de hacer una guerra. ${ }^{35}$ Bajo esta forma de detención, el prisionero no necesita ser juzgado pero puede ser detenido de manera indefinida sin cargos. ${ }^{36}$ Debe sin embargo, al menos si es ciudadano de los

30. La Corte reconoció este desequilibrio en INS v. Lopez-Mendoza, 468 U.S. 1032, 1038-39 (1984) ("de acuerdo a la naturaleza civil del procedimiento, varias protecciones que se aplican en el contexto de un juicio penal no se aplican en una audiencia de deportación.").

31. Ver generalmente, por ejemplo, Gabriel J. Chin, Segregation's Last Stronghold: Race Discrimination and the Constitutional Law of Immigration [Último Bastión de la Segregación: Discriminación De Raza y el Derecho Constitucional Sobre Immigración], 46 U.C.L.A. L. REV. 1 (1998).

32. Ver Jonathan Hafetz, Stretching Precedent Beyond Recognition: The Misplaced Reliance on World War II Cases in the "War on Terror," [El Precedente se Extiende más allá de Reconocimiento: La Confianza Fuera de Lugar en la II Guerra Mundial, los casos en la "Guerra Contra el Terror'] 28 REV. LITIG. 365, 365 (2008).

33. Aunque la Obama elimina la etiqueta de "enemigo combatiente", ha afirmado similar (aunque más limitada) autoridad para detener a personas indefinidamente como "beligerantes enemigos no privilegiados" si son parte de o han apoyado sustancialmente a Al Qaeda, los talibanes, o fuerzas asociadas. Ver In re Guantánamo Bay Detainee Litigation, Respondents Memorandum Regarding The Government's Detention Authority Relative to Detainees Held at Guantánamo Bay [Litigio En Re Detenidos de la Bahía de Guantánamo, Memoranđum de Contestación en Relación a la Autoridad del Gobierno de Detención Relativa a los Detenidos de la Bahía de Guantánamo], en 1, No. 08-442 (2009), disponible en http://www.scotusblog.com/wp-content/uploads/2009/03/doj-detain-authority-3-13-09.pdf (última visita el 10 de marzo del 2012).

34. Hamdi v. Rumsfeld, 542 U.S. 507, 519 (2004).

35. Id.

36. Ver id. en 509 (sosteniendo que el debido proceso exige que se de sólo una "posibilidad real de impugnar la base fáctica de la detención a un ciudadano detenido"). 
Estados Unidos, recibir el proceso debido. ${ }^{37}$ Pero este proceso puede realizarse en un tribunal militar debidamente constituido y que, incluso si adopta la forma de una audiencia en la corte federal, se tienen que tener en cuenta las preocupaciones del gobierno sobre seguridad nacional a través, por ejemplo, de pocas restricciones en testimonios y menor carga de la prueba que en un procedimiento legal. ${ }^{38}$ Aunque la corte en el caso Hamdi advirtió del peligro de ampliar este paradigma más allá de los parámetros de un prisionero capturado en el campo de batalla (en el caso de Hamdi, en Afganistán), el concepto de una "guerra contra el terror"-y, por extensión autoridad de detención que se extiende más allá de las capturas en los campos de batallas-ha seguido ganando aceptación entre los tribunales, los legisladores, y el público. ${ }^{39}$

En teoría, los ciudadanos y los no ciudadanos pueden ser detenidos como "combatientes enemigos." ${ }^{, 40} \mathrm{Hamdi}$, por supuesto, era un ciudadano estadounidense. ${ }^{41}$ En la práctica, si embargo, el poder de detención del "combatiente enemigo" ha sido utilizado casi exclusivamente en contra de los no ciudadanos, mientras que los ciudadanos sospechosos de terrorismo, en todo caso, han sido enjuiciados ante una corte federal. ${ }^{42}$ El principal asesor del presidente Obama en la lucha contra el terrorismo ha declarado que la administración no va a buscar detener a los ciudadanos estadounidenses fuera del sistema de justicia penal (no obstante Hamdi). ${ }^{43}$ Para los detenidos que enfrentan ser procesados en una comisión militar por crímenes de guerra, la dicotomía entre los ciudadanos y los no ciudadanos ha sido durante mucho tiempo explícita: el 13 de noviembre del 2001 el presidente Bush decretó una orden ejecutiva estableciendo las comisiones

37. Id.

38. Ver id. en 538-39.

39. Ver, por ejemplo, National Defense Authorization Act for Fiscal Year 2012 [Ley de Autorización de la Defensa Nacional para el A año Fiscal 2012], H.R. 1540, 112th Cong. § 1021 (2011).

40. Ver Norma C. Bay, Executive Power and the War on Terror [Poder Ejecutivo y la Guerra contra el Terrorismo], 83 DENV. U. L. REV. 335, 338 (2005) (reconociendo el potencial de la detención indefinida de ciudadanos categorizados como "combatientes enemigos", como resultado del cambio de paradigma del tratamiento del terrorismo como si fuera un problema militar).

41. Id. Ver también Padilla v. Hanft, 423 F.3d 386, 386 (4th Cir. 2005).

42. Únicamente dos ciudadanos Americanos han sido detenidos como "combatientes enemigos" en la "guerra sobre el terror": Yaser Hamdi y Jose Padilla. Por el contrario, varios miles de extranjeros han sido detenidos como "combatientes enemigos," excluyendo aquéllos detenidos en Iraq.

43. Ver Remarks by John O. Brennan [Palabras de John O. Brennan], Asistente del Presidente del Departamento de Homeland Security y Counterterrorism, en el Brennan Center for Justice en NYU School of Law Symposium (18 de marzo del 2011), disponible en http:/www.brennancenter.org/ content/resource/remarks_by_john_brennan_at_brennan_center_symposium/ (última visita el 30 de marzo 30 del 2012). 
militares, ${ }^{44}$ y los decretos de comisiones militares de 2001 y 2009 que le siguieron, se refieren explícitamente sólo a los no ciudadanos. ${ }^{45}$ Las comisiones fueron creadas originalmente para juzgar a los sospechosos de terrorismo sin las protecciones del sistema de justicia penal. ${ }^{46}$ Aunque las comisiones actuales ofrezcan ahora más garantías que las anteriores, estas todavía no le ofrecen a los acusados las mismas protecciones que recibirían en un juicio federal. ${ }^{47}$

Si un individuo que no es ciudadano es acusado de un delito, incluso un delito de terrorismo, esta facultado para tener acceso a la Quinta y Sexta Enmienda de derechos, de la misma manera que un ciudadano frente a la acusación. ${ }^{48}$ Sin embargo, como los no ciudadanos pueden ser detenidos fuera del sistema de justicia penal, estas protecciones son otorgadas a discreción del gobierno, en dependencia si elige proceder bajo el marco de "la ley de la guerra," al menos con respecto a la categoría de casos abarcadas en este marco. ${ }^{49}$ En resumen, después del 11/09 en el contexto de la "ley de la guerra" la detención y los procesamientos por las comisiones militares sirven una función similar a la clasificación de deportación en el contexto de deportación de inmigración: creando una alternativa y un foro de menos derechos de protección para la adjudicación

44. En 2006, el Tribunal Supremo invalidó una orden ejecutiva de Bush, decidiendo que las comisiones militares que él había creado no constaban con autorización del congreso. Ver Hamdan $v$. Rumsfeld, 548 U.S. 557, 568 (2006). Sin embargo, el tribunal no rechazó la creación de nuevas comisiones militares o comisiones que se aplicaran solo a extranjeros, como la Ley de Comisiones Militares de 2006 y 2009.

45. Military Commissions Act of 2006 [Ley Sobre Comisiones Militares de 2006], Pub. L. No. 109-366, 120 Stat. 2600 (2006) [en lo sucesivo MCA del 2006]; Military Commissions Act of 2009 [Ley Sobre Comisiones Militares de 2009], Pub. L. No. 111-84, 123 Stat. 2190 (2009) [en lo sucesivo MCA del 2009].

46. Ver Detention, Treatment, and Trial of Certain Non-Citizens in the War Against Terrorism [Detención, Tratamiento y Enjuiciamiento de Ciertos Extranjeros en la Guerra Contra el Terrorismo], 66 Fed. Reg. 57,833 (13 de noviembre del 2001).

47. Las comisiones, por ejemplo, ofrecen menos garantías contra el uso de pruebas de rumores. Ver HAFETZ, supra nota 12, en 241-42.

48. Ver, por ejemplo, Wong Wing v. United States, 163 U.S. 228, 241 (1896). Las protecciones que la cuarta enmienda ofrece, sin embargo, pueden variar entre ciudadanos y extranjeros, al menos si la captura ocurre fuera de los Estados Unidos. Ver United States v. Verdugo-Urquidez, 494 U.S. $259,274-75(1990)$.

49. Ver Ashley C. Pope, After Guantánamo: Legal Rights of Foreign Detainees Held in the United States in the "War on Terror" [Después de Guantánamo: Derechos Legales de los Extranjeros Recluidos en los Estados Unidos en la Guerra “Contra el Terrorismo'], 34 FORDHAM INT'L L.J. 504, 532 (2011) (detallando la disminución de los derechos procesales de la detención bajo la ley de guerra y del marco de acción judicial). 
de los derechos de los no ciudadanos que enfrentan severas privaciones de libertad.

\section{Restringir el acceso a las cortes y reexaminar el alcance}

Desde finales del siglo diecinueve, los poderes políticos han intentado repetidamente de restringir la revisión de un tribunal federal por encima de las decisiones administrativas de deportar o excluir a los no ciudadanos de los Estados Unidos. ${ }^{50}$ Aunque los tribunales han mantenido algunas formas de revisión judicial, especialmente sobre las decisiones de deportación, esa revisión se ha centrado en conservar más los procedimientos que los derechos sustanciales. ${ }^{51}$ Además, esas protecciones de procedimientos, basadas en la Cláusula de Debido Proceso, muchas veces han sido limitadas en alcance e intensidad. ${ }^{52}$ Ambos, la deportación de naturaleza civil y los plenos poderes del gobierno sobre la inmigración han justificado las limitaciones en los derechos de los no ciudadanos que enfrentan la deportación de los Estados Unidos. ${ }^{53}$

Las medidas del Congreso durante la última década dirigidas a "los criminales no ciudadanos" siguen este patrón general. ${ }^{54}$ En 1996, por

50. Ver Gerald L. Neuman, Jurisdiction and the Rule of Law After the 1996 Immigration Act [Jurisdicción y el Estado de Derecho Despues de La Ley de Inmigración de 1996], 113 HARV. L. REV. 1963, 1967 (2000) (señalando los repetidos intentos por parte del Congreso para establecer finalidad y limitar la revisión judicial de las decisiones de los oficiales de inmigración).

51. Ver Kanstroom, supra nota 28, en 1903; Hiroshi Motomura, The Curious Evolution of Immigration Law: Procedural Surrogates For Substantive Constitutional Rights [La Curiosa Evolución del Derecho Sobre Inmigración: Substitutos Procesales de Derechos Constitucionales Sustantivos], 92 Colum. L. ReV. 1625, 1628 (1992). Ver también Lenni B. Benson, Back to the Future: Congress Attacks the Right to Judicial Review of Immigration Proceedings [Vuelta al Futuro: el Congreso Ataca el Derecho a la Revisión Judicial en Procesos Sobre Inmigración], 29 CONN. L. REV. 1411, 1418 (1997).

52. Por ejemplo, cuando el Tribunal Supremo decidió que los extranjeros que se enfrentaban a la deportación tenían derecho a un proceso con garantias, el Tribunal también decidió que la vista que se había llevado a en el caso en cuestión satisfacía los requerimientos de un proceso con todas las garantías de la ley, aunque el extranjero argumentaba que sólo había recibido una notificación informal de la vista y que no entendía ni el idioma del proceso ni la naturaleza de los cargos mantenidos contra ella. Ver Yamataya v. Fisher, 189 U.S. 86, 101-02 (1903). Ver generalmente Chacon, supra nota 8, en 1868-69, n.231.

53. Ver generalmente Gerald L. Neuman, Habeas Corpus, Executive Detention, and Alien Removal [Habeas Corpus, Detención Ejecutiva, y Deportación de Extranjeros], 98 COLUM. L. REV. 961 (1998).

54. Ver Vashti D. Van Wyke, Retroactivity and Immigrant Crimes Since St. Cyr: Emerging Signs of Judicial Restraint [Retroactividad y Crimenes de Inmigrantes desde Saint-Cyr: Las Señales Emergentes de la Restricción Judicial], 154 U. PA. L. REV. 741, 741 (2006) (señalando que el propósito central de AEDPA e IIRIRA es facilitar la deportación de inmigrantes condenados través de la restricción y eliminación de la Sección 212 (c) mecanismo de exención). 
ejemplo, la Ley Antiterrorismo de la Pena de Muerte Efectiva (AEDPA) ${ }^{55}$ y la Ley de reforma de inmigración ilegal y responsabilidad del inmigrante (IIRIRA) ${ }^{56}$ pretendían privar a los tribunales federales de jurisdicción para revisar la deportación de no ciudadanos condenados por ciertos delitos. ${ }^{57}$ $\mathrm{Al}$ mismo tiempo, el Congreso ha ampliado de manera significativa la categoría de extranjeros que podrían ser deportados, incluyendo a aquellos que han cometido delitos relativamente menores o delitos en el pasado, ${ }^{58}$ mientras elimina una forma crítica de la facultad de ayuda discrecional de deportación, mediante la cual un juez tenía la facultad de conceder una exención de deportación basada en una consideración individualizada de intereses humanitarios, tales como el tiempo de esa persona no ciudadana en los Estados Unidos, las relaciones familiares, y los lazos con la comunidad. $^{59}$

En junio de 2001, la Corte suprema sostuvo que la eliminación de toda revisión judicial de las decisiones de deportación incluido el examen de hábeas corpus, plantearía graves problemas constitucionales bajo la Cláusula de Suspensión y de acuerdo a la interpretación de las leyes de 1996 de no eliminar la revisión del hábeas. ${ }^{60}$ La corte también concluyó que la disposición de eliminar exenciones discrecionales de deportación no se aplicó de manera retroactiva a los no ciudadanos que se declararon culpables de un delito criminal y que habrían sido elegidos para una renuncia discrecional de deportación en el momento de su petición bajo la

55. Antiterrorism and Effective Death Penalty Act of 1996 [Ley Antiterrorista y de Pena de Muerte Efectiva de 1996], Pub. L. 104-132, 110 Stat. 1214 (codificada en su forma enmendada en secciones dispersas $8,15,18,19,21,22,25,28,40,42,49$ del U.S.C.) [en lo sucesivo AEDPA].

56. Illegal Immigration Reform and Immigrant Responsibility Act [Ley de Reforma a la Inmigración Ilegal y de Responsabilidad del Inmigrante], Pub. L. No. 104-208, 110 Stat. 3009-546 (codificada en su forma enmendada en secciones dispersas $3,6,7,8,13,16,26,29,30,31,32,33,40$, $41,42,43,44,45,46,47,48$ del U.S.C.) [en lo sucesivo IIRIRA].

57. Ver Emest A. Young, Constitutional Avoidance, Resistance Norms, and the Preservation of Judicial Review [Evitando la Constitucional, Normas de Resistencia, y la Preservación de la Revisión Judicial], 78 TEX L. REV. 1549, 1562 (2000) (identificando disposiciones de AEDPA e IIRIRA que tienen como objeto restringir la revisión judicial de las órdenes de deportación en casos de inmigración).

58. Ver Chacon, supra nota 8, en 1844-45 (debatiendo la expansión de la categoría de "delito agravado" y otros cambios).

59. IIRIRA eliminó la dispensa de deportación contemplada en la antigua sección 212(c) de la Ley de Inmigración y Nacionalidad ("INA"), reemplazándola por una medida mucho más limitada conocida como "cancelación de deportación" bajo INA sección 240A. Ver IIRIRA, supra nota 57, § 304(b); 8 U.S.C. $\S 1129$ b(a)-(b). Ver también Chacon, supra nota 8, en 1845-46 (debatiendo los cambios surgidos por las Leyes Sobre Inmigración de 1996). Durante los cinco años previos a 1996, la autoridad para conceder dispensas discrecionales había sido ejercida para prevenir la deportación de más de 10,000 extranjeros. Ver Padilla, 130 S. Ct. en 1480.

60. INS v. St. Cyr, 533 U.S. 289, 301 (2001). 
ley entonces en efecto. ${ }^{61}$ Mientras el Congreso continuó limitando la revisión de deportación a través de la ley de identificación REAL, algunos casos de revisión judicial se mantienen. ${ }^{62}$ Esta revisión, sin embargo ha hecho poco para cambiar las consecuencias legales cada vez más duras impuestas a no ciudadanos debido a la criminalización de violaciones de inmigración, extensión de delitos separables, y restricciones de carácter discrecional atenuantes. ${ }^{63}$ Como antes, las intervenciones judiciales han sido dirigidas principalmente a garantizar protecciones de procedimiento en lugar de abordar políticas más amplias. ${ }^{64}$ En último término, por ejemplo, la Corte suprema sostuvo en el caso de Padilla v. Kentucky que el derecho en la Sexta Enmienda de tener representación adecuada aplica al asesoramiento dado por abogados de defensa criminal a los no ciudadanos respecto a las consecuencias de inmigración de una condena criminal. ${ }^{65}$ Aunque Padilla puede llegar a mitigar el impacto de las leyes que imponen consecuencias severas de inmigración por convicciones criminales muchas veces menores, esto no va a afectar la dirección total de la política de inmigración. ${ }^{66}$

Las decisiones judiciales que implican a detenidos en la "guerra contra el terror" han seguido una trayectoria similar, con tribunales que se resisten a los esfuerzos por eliminar la revisión judicial y ofrecer algunas garantías

\section{Id. en 326.}

62. Ver Nancy Morawetz, Back to the Future: Lessons Learned from Litigation over the 1996 Restrictions on Judicial Review [Vuelta al Futuro: Lecciones Aprendidas de los Litigios Sobre las Restricciones en la Revisión Judicial de 1996], 51 N.Y.L. SCH. L. REV. 113, 123-29 (2006-2007) (describiendo cómo la ley REAL ID optimiza y restringe la revisión judicial de varios aspectos en casos de deportación); Aaron G. Leiderman, Channeling the Constitution's Most Important Human Right: Judicial Review of Mixed Questions under the REAL ID Act [Canalizando el Derecho Humano Más Importante de la Constitución: Revisión Judicial de Questiones Mixtas Bajo la Ley REAL ID], 106 COLuM. L. REV. 1367, 1373-76 (2006) (describiendo cómo la Ley REAL ID amenaza con restringir la revisión judicial mediante la canalización de la revisión en los tribunales de apelación).

63. Ver Juliet P. Stumpf, Doing Time: Crimmigation Law and the Perils of Haste [Haciendo Tiempo: Ley de Crinmigración y los Peligros de la Precipitación], 58 UCLA L. REV. 1705, 1734 (2011) (identificando las consecuencias de la inmigración que son especialmente duras de, entre otros crímenes, delitos graves y ciertos delitos de drogas).

64. Ver generalmente Hiroshi Motomura, The Curious Evolution of Immigration Law: Procedural Surrogates for Substantive Constitutional Rights [La Curiosa Evolución de la Ley de Inmigración: Los Sustitutos de Procedimiento Para los Derechos Constitucionales], 92 CoLUM. L. REv. 1625 (1992).

\section{Padilla, 130 S. Ct. en 1482.}

66. Ver McGregor Smyth, From "Collateral" to "Integral": The Seismic Evolution of Padilla v. Kentucky and Its Impact on Penalties Beyond Deportation [Desde "Colateral" a "Integral": La Evolución Sísmica de Padilla v. Kentucky y su Impacto Sobre las Sanciones Más allá de la Deportación], 54 How. L.J. 795, 807-09 (2011) (discutiendo el impacto potencial de Padilla). 
básicas de procedimientos, a falta de no cuestionar las políticas sustanciales que limitan los derechos de los no ciudadanos en nombre de la seguridad nacional. ${ }^{67}$ En enero de 2002, los Estados Unidos comenzaron a llevar prisioneros a su base naval en la bahía de Guantánamo, Cuba. ${ }^{68} \mathrm{El}$ gobierno se resistió sistemáticamente a cualquier esfuerzo de los prisioneros allí de buscar un hábeas corpus de revisión de su detención. ${ }^{69}$ Argumentando que como no ciudadanos mantenidos fuera del territorio de los Estados Unidos, los prisioneros en Guantánamo no tenían ningún derecho a la revisión judicial de su confinamiento. ${ }^{70}$ Por lo tanto, desde el principio, la condición de ciudadano, en conjunción con la localización territorial, sirvió como base para negar a los extranjeros el acceso a los tribunales estadounidenses. ${ }^{71}$

En cada una de sus tres decisiones sobre los "combatientes enemigos" en Guantánamo, la Corte suprema ha mantenido la jurisdicción de hábeas corpus federal sobre las detenciones. ${ }^{72}$ En el caso de Rasul v. Bush ${ }^{73}$ y Hamdan v. Rumsfeld, ${ }^{74}$ las dos primeras decisiones sobre detenidos en

67. Ver Jenny S. Martinez, Process and Substance in the 'War on Terror' [El Proceso y la Sustancia en la 'Guerra Contra el Terrorismo ], 108 CoLUM. L. REv. 1013, 1029-32 (2008).

68. Ver A Nation Challenged: Guantánamo Bay; In Cuba, Muted Acceptance Greets Presence of Prisoners [Una Nación Desafiada: La Bahía de Guantánamo; en Cuba, La Aceptación Silenciada Saluda la Presencia de los Reclusos], N.Y. TimEs (14 de enero del 2002), disponible en http://www.nytimes.com/2002/01/14/world/nation-challenged-guantanamo-bay-cuba-muted-acceptancegreets-presence-prisoners.html?ref-guantanamobaynavalbasecuba (última visita el 30 de marzo del 2012).

69. Ver Richard H. Fallon, Jr., The Supreme Court, Habeas Corpus, and the War on Terror: An Essay on Law and Political Science [La Corte Suprema de Justicia, Habeas Corpus, y la Guerra Contra el Terrorismo: Un Ensayo Sobre Derecho y Ciencias Politicas], 110 ColuM. L. REv. 352, 356 (2010) (explicando que no fue sino hasta el 2004, a través de la decisión Rasul, que la Corte reconoció que la jurisdicción federal sobre los detenidos de Guantánamo existía en el Estatuto General de hábeas corpus).

70. Ver Jonathan HafeTz, Habeas CoRpus afTER 9/11: CONFronting AMERICA'S New Global Detention System [HABEAS Corpus Desput́s DEL 9/11: ENFrENTANDo al Nuevo SISTEMA DE DETENCIÓN GLOBAL DE AMÉRICA]118 (2011) (describiendo el argumento del gobierno en el caso Rasul que "los extranjeros retenidos fuera del territorio soberano de los Estados Unidos no tenian derecho al hábeas corpus o de otras protecciones constitucionales").

71. Id. ("El gobierno de Bush se basó principalmente en Johnson v. Eisentrager, argumentando que se estableció una regla categórica restringiendo el ejercicio de la revisión del hábeas corpus sobre la detención de cualquier ciudadano extranjero capturado y retenido en el extranjero.") (Énfasis añadido).

72. Ver Marc D. Falkoff, Back to Basics: Habeas Corpus Procedures and Long-Term Executive Detention [Volviendo a lo Básico: Procedimientos de Habeas Corpus y la Detención Prolongada Ejecutiva], 86 DENV. U. L. REv. 961, 991-93 (2009).

73. Rasul v. Bush, 542 U.S. 466, 484 (2004).

74. Boumedine v. Bush, 553 U.S. 723, 843-44 (2008). 
Guantánamo, la Corte mantuvo jurisdicción hábeas por motivos legales. ${ }^{75}$ En Boumediene v. Bush, la tercera decisión de la trilogía, la Corte determinó que el esfuerzo del Congreso por privar a los tribunales de jurisdicción violó la Cláusula de Suspensión de la constitución. ${ }^{76}$ Desde que el caso Boumediene fue decidido hace más de tres años, las cortes de distritos han emitido sesenta decisiones de hábeas en los casos de detenidos en Guantánamo y el circuito de D.C. ha emitido trece opiniones, dirigiéndose a una serie de cuestiones relativas a la legalidad de la detención de los no ciudadanos en Guantánamo. ${ }^{77}$

En general, el litigio de hábeas de los detenidos en Guantánamo, ha cedido algunas líneas de base de protecciones en los procedimientos para los detenidos, incluso el derecho a una audiencia ante un juez federal, el derecho de presentar pruebas en su defensa, refutar las pruebas del gobierno y asesoramiento de un abogado. ${ }^{78}$ Estas garantías de procedimientos, sin embargo, han sido limitadas en algunos aspectos importantes. Al gobierno le ha sido permitido contar, de manera extensiva y muchas veces exclusiva, con testimonios; negar a los detenidos el acceso a la información y se les ha impuesto sólo el estándar de preponderancia de pruebas, un estándar inferior a otros casos no criminales donde los individuos son privados de su libertad. ${ }^{79}$ Además varias de las decisiones tomadas por el circuito de D.C., han tenido una visión particularmente estrecha sobre los derechos hábeas de los detenidos, que exigen una deferencia a las pruebas del gobierno y abogan incluso por un estándar menor de pruebas de preponderancia. ${ }^{80}$ Las resoluciones hábeas después de Boumediene, además, han mantenido la

75. Rasul, 542 U.S. en 481 (aplicando 28 U.S.C. $\$ 2241$ (c)(1) (2006)); Hamdan, 548 U.S. at 567 (aplicando 10 U.S.C. $\$ 821$ (2006)).

76. Boumediene v. Bush, 553 U.S. 723, 792 (2008).

77. Ver Jonathan Hafetz, Calling the Government to Account: Habeas Corpus in the Aftermath of Boumediene v. Bush [Pidiendo al Gobierno Rendir Cuentas: Habeas Corpus Tras las Secuelas Del Caso de Boumedine v. Bush], 57 WAYNE L. REV. (2012) (forthcoming).

78. Id. en _ (describiendo el proceso de habeas corpus tras el caso Boumediene); Stephen I. Vladeck, The D.C. Circuit Afier Boumediene [El Tribunal del Distrito Federal del Circuito Después de Boumedine], 42 SETON HALl L. REV. 12 (2011) (lo mismo); Baher Azmy, Executive Detention, Boumediene and the New Common Law of Habeas [Detención Ejecutiva, Boumedine y el Nuevo Derecho Común del Habeas], 95 Iowa L. REv. 445, 460-61 (2010) (lo mismo).

79. Ver Addington v. Texas, 441 U.S. 418, 433 (1979) (exigiendo pruebas claras y convincentes en apoyo del compromiso civil); Woodby v. INS, 385 U.S. 276, 286 (1966) (exigiendo pruebas claras y convincentes en apoyo de la deportación).

80. Ver, por ejemplo, Al-Adahi v. Obama, 613 F.3d 1102, 1103 (D.C. Cir. 2010). Ver también Esmail v. Obama, No. 10-5282, 2011 WL 1327701, at *3 (D.C. Cir. Apr. 8, 2011) (Silberman, J,, concurriendo) (resaltando que un juez no ordenará ni deberá ordenar la liberación de un detenido en Guantánamo si él o ella cree que "posiblemente sea un miembro de al Qaeda o un partidario activo") (énfasis añadido). 
autoridad del presidente para detener indefinidamente en Guantánamo a los no ciudadanos bajo custodia militar, sin cargos ni juicio. ${ }^{81}$ Notablemente, las cortes se han negado a limitar la autoridad del presidente a las detenciones militares en el campo de batalla, ${ }^{82}$ ampliando la autoridad reconocida en el caso de Hamdi para justificar un sistema de facto de detención preventiva en Guantánamo que le permite la detención de individuos basada en la supuesta membresía de estos en Al Qaeda o en los grupos asociados. ${ }^{83}$ En la década pasada la jurisprudencia respecto a los "combatientes enemigos" se asemeja a la ley de inmigración del siglo pasado en sus líneas fundamentales: mantener limitado el acceso a las cortes y las protecciones de debido proceso manteniendo el amplio poder del gobierno sobre la libertad de los no ciudadanos. ${ }^{84}$

\section{Seguridad como poder para otros objetivos}

La seguridad durante mucho tiempo ha sido invocada como una razón fundamental para las restricciones de inmigración. Desde mediados de los años noventa, el terrorismo y la inmigración han sido cada vez más asociados. ${ }^{85}$ El presidente Clinton, por ejemplo, explotó el "terror en

81. Ver, por ejemplo, Uthman v. Obama, 637 F.3d 400, 405 (D.C. Cir. 2011) (prueba de la asociación con otros miembros de al Qaeda puede ser probativo de su la pertenencia al grupo terrorista); $A l$-Adahi, 613 F.3d en 1108 (concluyendo que la prueba circunstancial, como habiendo estado en una casa de invitados de al Qaeda, es "poderosa", si no "aplastante" prueba de que el individuo forma "parte de" al Qaeda y por tanto sujeto de detención bajo el AUMF).

82. Ver, por ejemplo, Salahi v. Obama, 625 F.3d 745, 752 (D.C. Cir. 2010) (invirtiendo y devolviendo la concesión del habeas corpus por parte del Tribunal del distrito federal al solicitante capturado en Mauritania y que por tanto nunca estuvo en un campo de batalla ni tomó parte en hostilidades contra los Estados Unidos o las fuerzas aliadas durante los conflictos armados contra al Qaeda).

83. Ver Kristine A. Huskey, Guantanamo and Beyond: Reflections on the Past, Present, and Future of Preventive Detention [Guantánamo y Más Allá: Reflexiones Sobre el Pasado, Presente y Futuro de la Detención Preventiva], 9 U.N.H. L. REV. 183, 201-02 (2011) (hanblando sobre el fracaso del circuito de D.C. para limitar los límites del campo de batalla, contribuyendo a la creación de un régimen de detención preventivo).

84. Ver generalmente Faiza W. Sayed, Challenging Detention, Why Immigrant Detainees Receive Less Process Than "Enemy Combatants" and Why They Deserve More [Detención de un Reto, ¿Por Qué los Inmigrantes Detenidos Reciben Menos Proceso Que los “Combatientes Enemigos" y Por Qué Ellos Merecen Más], 111 ColuM. L. REV. 1833 (2011) (comparando historias respectivas y el estado actual de los inmigrantes detenidos y presuntos "combatientes enemigos" detenidos).

85. Ver Dan Eggen, Tough Anti-Terror Campaign Pledged; Ashcroft Tells Mayors He Will Use New Law to Fullest Extent [Dura Campaña Antiterrorista Prometida; Ashcroft Dice a los Alcaldes que Usará Nueva Ley a Toda Medida], WASH. POST, 26 de octubre del 2001, en Al ("Que los terroristas entre nosotros estén advertidos... [s]i se quedan más de tiempo de lo que les permite su visa hasta por un dia, se les arrestará."). 
América" a los extranjeros para justificar un mayor control de las fronteras. ${ }^{86}$ Aunque el atentado con bombas perpetrado en la ciudad de Oklahoma en 1993-fue una ataque terrorista cometido por ciudadanos americanos-AEDPA se convirtió en un vehículo para la aprobación de varias medidas anti-inmigrantes, incluidas las disposiciones para la deportación acelerada de no ciudadanos. ${ }^{87}$ Tras los ataques del 11/09, el congreso decretó la Ley Patriota de EEUU, que contiene varias disposiciones dirigidas a los no ciudadanos, incluyendo la ampliación de las causas para la deportación sobre la base de aquellas personas que apoyan las actividades terroristas y permitiendo la detención indefinida de los extranjeros sospechosos de terrorismo que no podían ser deportados del pais. ${ }^{88}$ La Ley de la Identificación Real siguió esta tendencia, ampliando la definición de "organización terrorista" para de esta manera abarcar otras conductas criminales no relacionadas con el terrorismo. ${ }^{89}$

Esta medidas parecen tener poco que ver en lo que concierne a seguridad nacional. Sólo una pequeña fracción de las deportaciones anuales están basadas en razones de seguridad nacional -y esa cantidad ha descendido después del 11/09. ${ }^{90}$ A pesar de que gran parte de la política de inmigración está basada en la retórica de la seguridad, la deportación continúa siendo la herramienta principal utilizada para los no ciudadanos que han cometido violaciones de inmigración o de aquellos que han cometido delitos criminales separables. ${ }^{91}$ Las dos últimas décadas de la ley de inmigración subrayan hasta que punto la seguridad nacional sirve como poder para establecer medidas que restrinjan los derechos de los no ciudadanos sin servir a los fines de seguridad. ${ }^{92}$

86. Ver President William Jefferson Clinton, Conferencia de Prensa, 27 de julio de 1993.

87. Ver Chacon, supra nota 8, en 1852.

88. Uniting and Strengthening America by Providing Appropriate Tools Required to Intercept and Obstruct Terrorism Act of 2001 [Ley Para Unir y Fortalecer América al Proporcionar las Herramientas Necesarias Para Interceptar y Obstruir el Terrorismo del 2001], Pub. L. No. 107-56, §§ 376, 411, 115 Stat. 272 (2001) (expandiendo la definición de "apoyo material al terrorismo" para incluir, por ejemplo, acciones que incluyan el uso de cualquier "instrumento peligroso" para cualquier propósito distinto a un "mero beneficio económico personal"); $i d$. $\S 412$ (autorizando la detención indefinida de extranjeros sospechosos de ser terroristas bajo especificas circunstancias).

89. REAL ID Act of 2005 [Ley REAL ID del 2005], Pub. L. No. 109-13, § 103, 119 Stat. 302, 308 (codificada con enmiendas en 8 U.S.C. § 1103).

90. Ver Chacon, supra nota 8, en 1860.

91. Id. en 1861.

92. Ver Kevin R. Johnson, It's the Economy, Stupid: The Hijacking of the Debate Over Immigration Reform By Monsters, Ghosts, and Goblins (Or the War on Drugs, War on Terror, Narcoterrorists, Etc.) [Es la Economía, Estúpido: El Secuestro del Debate Sobre la Reforma de Inmigración por Monstruos, Fantasmas y Duendes (o de la Guerra Contra las Drogas, Guerra Contra 
El trato a los "combatientes enemigos" después del 11/09 ilustra una desconexión similar. Después del 11/09, por ejemplo, los Estados Unidos llevaron cientos de prisioneros a Guantánamo para someterlos a interrogatorios y continuaron bajo detención. ${ }^{93}$ En los primeros momentos, oficiales militares y de la inteligencia, reconocieron que muchos de los prisioneros en Guantánamo ni significaban una amenaza para los Estados Unidos ni tenían información valiosa que ofrecer. ${ }^{94}$ "En muchos casos habíamos capturado a los tipos más lentos en el campo de batalla. ${ }^{95}$ Literalmente encontramos a los tipos que le habían pegado un tiro en el trasero," comentó un oficial del Pentágono responsable de ayudar a establecer los primeros tribunales de crímenes de guerra en la base naval. ${ }^{96}$

Los funcionarios de la administración Bush justificaron las detenciones "calificando a los prisioneros como lo peor de lo peor" y afirmando que Guantánamo era vital para la seguridad de América. ${ }^{97}$ Estas explicaciones, sin embargo, en muchas ocasiones enmascaraban otras razones para las detenciones, incluyendo la hostilidad para procesar a los prisioneros en cortes federales, dificultades en devolver a los prisioneros a sus países de orígenes o repatriarlos a terceros países y el deseo de mostrar una imagen de mano dura contra el terrorismo. ${ }^{98}$ Mientras tanto, Guantánamo ha sufrido críticas fulminantes dentro y fuera del país. ${ }^{99}$

Finalmente, surgió un consenso político acerca de la idea de cerrar Guantánamo. Durante la campaña presidencial de 2008, candidatos de

el Terrorismo, Narcoterroristas, Etc.)], 13 CHAP. L. REv. 583, 592-600 (argumentando que la fusión de la ley de inmigración y la seguridad nacional es más un reflejo de los fines políticos que de la importancia de la inmigración en la llamada "guerra contra el terror").

93. Más de 775 prisioneros en total fueron llevados a Guantánamo; 171 todavía permanecen alli.

94. Ver HAFETZ, supra nota 8, en 151 ("incluso basado en alegaciones del gobierno que no han sido comprobadas, la mayoría de los prisioneros no eran terroristas peligrosos, y muchos eran totalmente inocentes").

95. Joseph Margulies, Guantánamo and the abuse of Presidential Power [Guantánamo y el abuso del Poder Presidencial] 70 (2006) (citando al Teniente Coronel Thomas Berg).

96. Id.

97. HAFETZ, supra, nota 12, en 134.

98. Los casos de los diecisiete detenidos Uighur, los miembros de la minoría musulmana perseguida en China desde hace mucho tiempo en Guantánamo, tal vez es el mejor ejemplo de estas dificultades y deseos. Ver id. en 248-50.

99. Ver id. en 155 (citando las críticas); Johan Steyn, Guantánamo Bay: The Legal Black Hole, Speech at the 27th FA Mann Lecture [Guantánamo: El Agujero Negro Legal, Discurso en la Conferencia 27 de FA Mann] (Brit. Inst. of Int'l \& Comp. Law, 25 de noviembre del 2003), disponible en http://www.oslaw.com/itow/source_files/Steyn\%20speech.pdf (última visita el 25 de marzo del 2012). 
ambos partidos dijeron que Guantánamo debería cerrarse. ${ }^{100}$ (El presidente Bush previamente había expresado su deseo de cerrar la prisión de ser posible). ${ }^{101}$ Como se explica más adelante, el fracaso subsecuente de la administración de Obama de cerrar la prisión subraya el vacío entre la retórica y la realidad respecto a la seguridad. Esto también arroja luz sobre cómo los temas de la ley de inmigración continúan emergiendo en el debate público y legal sobre la "guerra contra el terrorismo."

\section{EL FRACASO DE NO CERRAR GUANTÁNAMO}

Después de su inauguración, el presidente Obama, emitió una directiva ordenando el cierre del centro de detención de la bahía de Guantánamo en el periodo de un año. ${ }^{102}$ En explicación a su decisión, Obama subrayó la importancia de defender los principios constitucionales y los derechos humanos en la lucha contra el terrorismo. ${ }^{103}$ Además, Obama hizo la observación de que los posibles beneficios de Guantánamo habian sido superados por los daños que causó tanto a la seguridad de América como a sus valores. ${ }^{104}$ "[L]a existencia de Guantánamo, probablemente creó más terroristas alrededor del mundo que los que habían detenidos," comentó él. ${ }^{105}$

100. Carol Rosenberg, What to Do About Guantánamo Vexes Both Obama, McCain [Lo Que Hacer Sobre Guantánamo Exaspera Tanto a Obama como a McCain], MIAMI HERALD (13 de julio del 2008), http://www.mcclatchydc.com/2008/07/13/v-print/44087/what-to-do-about-guantanamovexes.html (última visita el 10 de marzo del 2012).

101. Bush: I Would Like to Close Guantánamo [Bush: Me Gustaría Cerrar Guantánamo], PRENSA ASOCIADA (8 de mayo del 2006), http://www.foxnews.com/story/0,2933,194634,00.html (última visita el 10 de marzo del 2012).

102. Exec. Order No. 13,492, 74 Fed. Reg. 4897 (22 de enero del 2009).

103. Ver Scott Shane, Obama Orders Secret Prisons and Detention Camps Closed [Obama Ordena Cerrar Prisiones Secretas y Campos de Detención], N.Y. TIMES (22 de enero del 2009), disponible en http://www.nytimes.com/2009/01/23/us/politics/23GITMOCND.html (última visita el 30 de marzo del 2012) (reportando la declaración de Obama durante la firma de la orden de que "nuestros ideales nos dan la fuerza y la autoridad moral para combatir el terrorismo").

104. Barack Obama, Remarks By the President on National Security [Barack Obama, Observaciones del Presidente SObre la Seguridad Nacional] (21 de mayo del 2009), disponible en $\mathrm{http}: / / w w w . w h i t e h o u s e . g o v / t h e \_p r e s s \_o f f i c e / R e m a r k s-b y-t h e-P r e s i d e n t-O n-N a t i o n a l-S e c u r i t y-5-21-09$ (última visita el 30 de marzo del 2012) ("En lugar de construir un marco duradero para la lucha contra Al Qaeda basandose en nuestros más profundos valores y tradiciones, nuestro gobierno defendió posturas que socavaban el estado de derecho ... en vez de mantenernos más seguros, la prisión de Guantánamo ha debilitado la seguridad nacional americana").

105. Ver Comunicado de Prensa, The White House, Remarks by the President On National Security [La Casa Blanca, Observaciones del Presidente Sobre la Seguridad Nacional] (21 de mayo del 2009). 
A más de dos años de su administración, el plan de Obama de cerrar Guantánamo se está desmoronando. Desde que ocupó la oficina de la presidencia, sólo sesenta y ocho prisioneros han sido transferidos de Guantánamo, sesenta y siete a sus países de orígenes o a un tercer país y uno (Ahmed Ghailani) enfrenta proceso criminal en los Estados Unidos, 171 prisioneros todavía permanecen en la base. ${ }^{106}$ Más importante aún, la legislación ahora previene al presidente de transferir a los detenidos de Guantánamo a los Estados Unidos y restringe su capacidad de transferirlos a terceros países. ${ }^{107}$ A la luz de estos acontecimientos, el secretario de defensa Gates ha reconocido que la perspectivas para el cierre de Guantánamo son "muy, muy bajas." los Estados Unidos están, desde un punto de vista práctico, mucho más lejos de cerrar el centro de detención ahora que cuando Obama tomó posesión su cargo.

Varios factores ayudan a desentrañar el plan de Obama para cerrar Guantánamo: la propia ambivalencia de Obama acerca de políticas más abarcadoras que tienen como base a Guantánamo, incluyendo la detención indefinida de sospechosos de terrorismo y el uso de comisiones militares; un contragolpe político que ha alterado la percepción pública de Guantánamo y allanó el camino para la legislación que previene transferir los detenidos de Guantánamo a los Estados Unidos; y las decisiones de la corte que interpretan estrictamente el papel judicial en la revisión de la legalidad de remediar las detenciones. Como se describe más adelante, los conceptos de inmigración ayudan a explicar cada factor.

\section{A. Guantánamo y el trato diferencial a los no ciudadanos}

A pesar de que Obama juró cerrar Guantánamo, él respaldo las dos características claves que son la base de la prisión: la detención indefinida de los sospechosos de terrorismo sin cargo y el procesamiento de los sospechosos de terrorismo por comisiones militares. ${ }^{109}$ En su discurso de

106. Ver The Guantánamo Docket [El Expediente de Guantánamo], N.Y. TIMEs (15 de febrero del 2012), disponible en http://projects.nytimes.com/guantanamo?ref=guantanamobaynavalbasecuba (última visita el 30 de marzo del 2012).

107. Ver National Defense Authorization Act for Fiscal Year 2012 [Ley de Autorización de la Defensa Nacional la para el Año Fiscal 2012], H.R. 1540, 112th Cong. $\S \S 1026-28$ (2011).

108. Charley Keyes, Gates: Prospects for Closing Guantánamo "Very, Very Low" [Gates: Perspectivas de cerrar Guantánamo "mıy, muy bajas'], CNN.COM (17 de febrero del 2011), http://articles.cnn.com/2011-02-17/politics/senate.gates.gitmo_1_terrorists-detention-center-militarycommissions?_s=PM:POLITICS (última visita el 10 de marzo del 2012).

109. Ver President Barack Obama, Remarks by the President on National Security at the National Archives, Washington, D.C. [Presidente Barack Obama, Declaraciones del Presidente sobre la Seguridad Nacional en los Archivos Nacionales, Washington, D.C.] (21 de mayo del 2009), disponible 
mayo del 2009 en el Archivo Nacional, Obama reiteró la importancia de cerrar Guantánamo y expresó la preferencia de su administración de procesar a los detenidos de Guantánamo en una corte federal donde fuera posible. ${ }^{10}$ Pero Obama también defendió la detención infinita y el enjuiciamiento militar de los detenidos de Guantánamo bajo la constitución, la ley federal, y la ley de la guerra. ${ }^{111}$ Obama, en otras palabras, no planeó tanto ponerle fin al sistema impuesto en Guantánamo sino mejorarlo: cerrar el centro de detención para reformar más que erradicar la arquitectura legal que lo ha apoyado. La administración de esta manera presentó una declaración más matizada de los poderes de detenciones militares del presidente bajo la AUMF; ${ }^{112}$ condujo una revisión inicial de los casos de todos los detenidos ${ }^{113}$ y creó un mecanismo permanente de mayor poder ejecutivo ${ }^{114}$ que ayuda a asegurar la aprobación de una nueva legislación para mejorar las comisiones militares. ${ }^{115}$ Mientras tanto, la administración continuó defendiendo agresivamente muchas detenciones de Guantánamo en el litigio de hábeas corpus en la corte federal.

en http://www.whitehouse.gov/the_press_office/Remarks-by-the-President-On-National-Security-5-2109/ (última visita el 30 de marzo del 2012).

110. Id. ("En primer lugar, siempre que sea posible, vamos a juzgar a los estadounidenses que han violado las leyes penales en los tribunales federales - los tribunales establecidos por la Constitución de los Estados Unidos").

111. Id. ("[Las comisiones militares] son un lugar apropiado para juzgar a detenidos por violaciónes de las leyes de guerra")

112. Ver Respondents' Memorandum Regarding the Scope of the Government's Detention Authority Relative to Detainees Held at Guantánamo Bay, In re Guantánamo Bay Detainee Litigation [Memorandum de Contestación en Relación a la Autoridad del Gobiemo de Detención Relativa a los Detenidos de la Bahía de Guantánamo, Litigio En Re Detenidos de la Bahía de Guantánamo], Misc. No. 08-442, presentado el 13 de marzo del 2009 (D.D.C.) (cambiando el estado de "enemigos combatientes" al de "enemigos beligerantes no privilegiados" y requiriendo que el soporte de un detenido a al Qaeda, los Talibanes, o las fuerzas asociadas, fuera "sustancial" para justificar su detención continuada).

113. U.S. DEP'T OF JUSTICE ET AL., FINAL REPORT: GUANTÁNAMO REVIEW TASK FORCE [INFORME FINAL: FuERZA ESPECIAL PARA LA REVISIÓNDE GUANTÁNAMO] (22 de enero del 2010), disponible en http://www.justice.gov/ag/guantanamo-review-final-report.pdf (última visita el 30 de marzo del 2012).

114. Ver Exec. Order No. 13,567, 76 Fed. Reg. 13277 (7 de marzo del 2011) (la creación de nuevas "Juntas de Revisión Periódica" para revisar los casos de los presos aprobados para continuar en detención). Ver también Charlie Savage, Detainee Review Proposal Is Prepared for Obama [Propuesta Para Revisión de Detenidos es Preparada para Obama], N.Y. TLMEs (21 de diciembre del 2010), disponible en http:/www.nytimes.com/2010/12/22/us/22gitmo.html (última visita el 30 de marzo del 2012).

115. MCA del 2009, supra nota 46 (modificando La Ley de Comisiones Militares del 2006). La MCA del 2009, por ejemplo, proporciona unas restricciones más amplias en el uso de la prueba basada en rumores y y la obtenida mediante coerción. Ver HAFETZ, supra nota 12, en 241-42 (debatiendo la MCA del 2009). 
La retención presidencial de detención indefinida y comisiones militares, no obstante estas reformas, ha socavado su plan de cerrar la prisión. Esto mantuvo la estructura legal que hizo Guantánamo factible al perpetuar una alternativa al proceso penal federal de sospechosos de terrorismo. ${ }^{116}$ También dejó una opción para ser ejercida como oposición política sobre la base del cierre de Guantánamo. ${ }^{17}$ Si el presidente no hubiera mantenido la posibilidad de detención indefinida o las comisiones militares, hubiera sido más difícil, por ejemplo, para la administración revertir la decisión original del fiscal general Eric Holder de procesar a Khalid Sheikh Mohammed y los otros co-conspiradores del 11/09 en una corte federal. ${ }^{118} \mathrm{La}$ administración inicialmente pudo haber mantenido la detención indefinida/ las comisiones militares como una opción porque creía que sería muy difícil procesar algunos casos debido a problemas legales o jurídicos de otro tipo. Pero también ejerció esa opción cuando algunos casos se hicieron muy complicados para procesar en cortes nacionales a consecuencia de la presión política, incluso si no hubiera existido ninguna barrera legal para lograr una condena.

Mantener el paradigma de Guantánamo también ha hecho el cierre parecer simbólico. ¿Cuál es la diferencia se han preguntado los comentaristas tanto de la izquierda como de la derecha, qué importa si los prisioneros siguen detenidos en Guantánamo en vez de en territorio de los Estados Unidos si van a ser sometidos a los mismas leyes militares basadas legalmente en el marco de la ley de la guerra? Separado de cualquier cambio mayor en la política, cerrar Guantánamo perdió su sentido de urgencia e incluso de necesidad. La retención de Obama de la detención indefinida y las comisiones militares ilustra un tema endémico de la ley de inmigración: cómo el desarrollo de mecanismos de menos derechos de protección de adjudicación para los no ciudadanos puede ser normalizado. A más de un siglo de la ley de inmigración se ha afianzado el principio de que aquellos que no son ciudadanos pueden ser deportados del país sin las mismas garantías constitucionales que ofrece un juicio penal, a pesar de la

116. MCA del 2009, supra nota 46 (modificando La Ley de Comisiones Militares del 2006). La MCA del 2009, por ejemplo, proporciona unas restricciones más amplias en el uso de la prueba basada en numores y y la obtenida mediante coerción. Ver HAFETz, supra nota 12, en 241-42 (debatiendo la MCA del 2009).

117. Charlie Savage, Closing Guantánamo Fades as a Priority [Cierre de Guantánamo se Desvanece Como una Prioridad], N.Y. TIMEs, 26 de junio del 2010, en A13.

118. Charlie Savage, In Reversal, Military Trials for 9/l1 Cases [A la Inversa, Litigios Militares Para Los Casos del 9/II], N.Y. TIMES, 4 de abril del 2011 , en A1. 
naturaleza potencialmente severa de la privación de libertad. ${ }^{119}$ Esto también ha contribuido a perpetuar la visión de que aquellos que no son ciudadanos son menos merecedores de las protecciones legales que tiene un ciudadano. Así mismo Guantánamo ha servido para atestiguar de manera similar el desarrollo de estructuras de adjudicación alternativas para los no ciudadanos que carecen de protecciones constitucionales importantes a pesar de las consecuencias extraordinarias que significan para los individuos afectados. Según estas estructuras se han institucionalizado en Guantánamo, ellos han incorporado el trato diferencial a los no ciudadanos, quienes, contrariamente a los ciudadanos estadounidenses, no necesitan ser enjuiciados en una corte cuando resultan sospechosos de actividades terroristas. ${ }^{120}$ Ellos también han ayudado a evitar el cierre de la prisión al legitimizar otra opción de procesamiento criminal para tratar a los no ciudadanos detenidos por los Estados Unidos en el curso de las operaciones contra el terrorismo. ${ }^{121}$

\section{B. El fracaso de reubicar a los detenidos en los Estados Unidos}

Cualquier plan factible para cerrar Guantánamo exige que el gobierno de los Estados Unidos reubique al menos algunos detenidos dentro de los Estados Unidos, en parte para lograr garantías diplomáticas dentro de otros países, especialmente en Europa, con quien los Estados Unidos confiaba en poder compartir gran parte de la responsabilidad que significa la reubicación. ${ }^{122}$ Los candidatos más obvios para la reubicación son los uigures, miembros de una minoría turca musulmana del noroeste de

119. Ver Andrea Lovell, The Proper Scope of Habeas Corpus Review in Civil Removal Proceedings [El Ámbito Concreto de la Revisión de Habeas Corpus en Procesos de Deportaciones Civiles], 73 WASH. L. REV. 459, 466-67 (1998).

120. HAFETZ, supra nota 12, en 211-13.

121. Id. en 241-42 (explicando la perpetuación del régimen de comisión militar defectuoso bajo la administración de Obama).

122. Ver Justin Blum, Some Chinese Guantanamo Detainees Likely to Be Released in U.S. [Algunos Chinos Detenidos en Guantánamo Podrian Ser Liberados en Estados Unidos], BLOOMBERG (3 de junio del 2009), http://www.bloomberg.com/apps/news?pid=newsarchive\&sid=apMw9EAc4PtU (última visita el 10 de marzo del 2012) (citando al antiguo consejero del Departamento de Estado John Bellinger III, el cual dijo que "sería imposible convencer a los países Europeos a aceptar el reasignamiento de los detenidos al menos que [los Estados Unidos] también admitan a algunos"). Ver también Proposed Budget Estimates for the Fiscal 2009 War Supplemental: Hearing Before the U.S. Appropriations Comm. [Propuesta de Presupuesto Para el Suplemento de Guerra del Año Fiscal 2009: Audiencia Ante el Comité de Consignaciones de Estados Unidos], 111 th Cong. (2009) (declaración de Robert M. Gates) (destacando la dificultad que el Departamento de Estado experimentará reasignando a los detenidos si Estados Unidos no acoge a ninguno). 
China. ${ }^{123}$ El gobierno de los Estados Unidos hace mucho tiempo que ha reconocido que no tiene ninguna base para detener a los uigures como "combatientes enemigos." 24 Aunque los uigures no podrían ser enviados de manera segura a China, donde pudieran ser encarcelados y sufrir otro tipo de persecución, se han realizado esfuerzos considerables para su reubicación dentro de los Estados Unidos e integrarlos en una comunidad uigur existente alli. ${ }^{125}$

La administración de Obama planeó originalmente traer varios uigures como parte de su esfuerzo para cerrar Guantánamo. ${ }^{126}$ Pero la administración abortó el plan frente a la primera señal de protesta. ${ }^{127}$ Esto no pudo entonces acallar el contragolpe político, conduciendo a una serie de medidas de asignaciones en el congreso impidiendo la liberación de cualquier detenido en Guantánamo dentro de los Estados Unidos. ${ }^{128}$ La falta de resistencia animó al Congreso, que posteriormente decretó la legislación que impide al presidente transferir a detenidos de Guantánamo a los Estados Unidos para cualquier finalidad, incluso la detención

123. Ver HAFETZ, supra nota 12, en 248-50 para una discusión de los casos de Uighur.

124. Qassim v. Bush, 382 F. Supp. 2d 126, 127-28 (D.D.C. 2005) (destacando la decisión de CSRT de que los detenidos Uighur no podían seguir siendo considerados "enemigos combatientes").

125. Ver William Glaberson, 6 Detainees Freed as Questions Linger [ 6 Detenidos Son Liberados Mientras Hay Preguntas Pendientes], N.Y. TIMEs (11 de junio del 2009), disponible en http:/www.nytimes.com/2009/06/12/world/12gitmo,html (última visita el 30 de marzo del 2012) (señalando el plan de la administración de Obama para reasentar a algunos de los Uighurs en Estados Unidos).

126. Id.

127. Jane Mayer, The Trial: Eric Holder and the Battle Over Khalid Sheikh Mohammed [El Juicio: Eric Holder y la Batalla Sobre Khalid Sheikh Mohammed], NEW YORKER (15 de febrero del 2010), http:/www.newyorker.com/reporting/2010/02/15/100215fa_fact_mayer (última visita el 10 de marzo del 2012).

128. Ver 2009 Supplemental Appropriations Act [Ley de Consignaciones Suplementarias del 2009], Pub. L. No. 111-32, 123 Stat. 1859 (promulgada el 24 de junio del 2009); Department of Homeland Security Appropriations Act [Ley de Consignaciones del Departamento de Seguridad Nacional], Pub. L. No. 111-83, 123 Stat. 2142 (2010); National Defense Authorization Act for Fiscal Year 2010 [Ley de Autorización de Defensa Nacional para el Año Fiscal 2010], Pub. L. No. 111-84, 123 Stat. 2190 (promulgada el 28 de octubre del 2009); Department of the Interior, Environment, and Related Agencies Appropriations Act [Ley de Consignaciones del Departamento de Interior, Medioambiental, y Agencias Conexas], Pub. L. No. 111-88, 123 Stat. 2904 (2010); Consolidated Appropriations Act [Ley de Consignaciones Consolidadas], Pub. L. No. 111-117, 123 Stat. 3034 (2010); Department of Defense Appropriations Act [Ley de Consignaciones del Departamento de Defensa], Pub. L. No. 111-118, 123 Stat. 3409 (2010). Ver también ANNA C. HENNING, GuANTÁNAMO DETENTION CENTER: Legislative ACTIVITY IN THE 111 TH CONGRESS [CENTRO DE DETENCIÓN DE GuantÁnamo: ACtividad Legislativa En El CONGReso 111] 2, 3-4 (Cong. Res. Serv., R 40754, 2010). 
continuada o proceso criminal judicial. ${ }^{129}$ Ante esta legislación, el plan de traer algunos de los detenidos en Guantánamo a cualquier instalación de los Estados Unidos para su confinamiento basado en la ley de la guerra bajo la Autorización para Uso de la Fuerza Militar (AUMF) ${ }^{130}$ fracasó, mientras los esfuerzos para procesar a otros detenidos de Guantánamo en tribunales de virtud del Artículo III fueron abandonados a favor de los procedimientos de las comisiones militares en Guantánamo. ${ }^{131}$

La reacción a reubicar a los uigures u otros detenidos de Guantánamo en los Estados Unidos refleja la asociación entre inmigrantes y el terrorismo que antecede por mucho al 11/09. Los uigures, como el gobierno reconoció, no representan ninguna amenaza a la seguridad nacional de Estados Unidos. ${ }^{132}$ Además, preocupaciones equitativas pesaban fuertemente a su favor: los uigures no vinieron buscando su admisión a los Estados Unidos sino que fueron en cambio traídos a la fuerza a Guantánamo y encarcelados alli; ellos iban a ser liberados en los Estados Unidos sólo porque no podían ser repatriados sin que corrieran peligro a su país de origen. ${ }^{133}$ Los uigures se convirtieron en el centro de atención de una amplia gama de sentimientos que asocian a los inmigrantes con el terrorismo. Ellos fueron representados como un grupo de extranjeros cuya presencia en suelo estadounidense pondría en peligro la seguridad del país. En efecto, la asociación entre inmigrantes y terrorismo ha probado ser tan poderosa que esto ha ayudado a conducir la legislación que excluye la transferencia de cualquier detenido de Guantánamo a los Estados Unidos, hasta para la detención indefinida. Uno de los estímulos detrás de esa legislación es el miedo a que una corte, ejerciendo su jurisdicción de hábeas constitucionalmente según el caso Boumediene, podría más fácilmente ordenar la liberación de un prisionero que fue ilegalmente detenido en una instalación estadounidense (después de haber sido transferido de Guantánamo) que si ese prisionero estuviera todavía detenido en Guantánamo. ${ }^{134}$ El cierre de Guantánamo fue representado como un debilitamiento de la capacidad de Estados Unidos para excluir a ciudadanos

129. Ver Ike Skelton National Defense Authorization Act for Fiscal Year 2011 [Ley de Autorización de Defensa Nacional Ike Skeiton Para el Año Fiscal 2011], H.R. 6523, 111th Cong. § 1031 (2010).

130. HAFETZ, supra nota 12, en 250.

131. Ver Richard A. Serrano, Obama to Resume Military Trials for Guantánamo Detainees [Obama Reanudará Juicios Militares Para los Detenidos de Guantánamo], L.A. TIMEs (8 de marzo del 2011), disponible en http://articles.latimes.com/2011/mar/08/nation/la-na-obama-guantanamo-20110308 (última visita el 30 de marzo del 2012).

132. Qassim, 382 F. Supp. $2 \mathrm{~d}$ en 127-28.

133. Ver Glaberson, supra nota 126.

134. HAFETZ, supra nota 12 , en 250. 
extranjeros en nombre de la seguridad nacional al abrirles la puerta para que entrasen a los Estados Unidos-el temor que anteriormente motivó la legislación en materia de inmigración destinada a la exclusión y deportación de los no ciudadanos para protegerse contra el terrorismo.

\section{La doctrina de poderes plenos y litigios de habeas en Guantánamo}

Las decisiones judiciales que abordan el poder de la Corte para ordenar la liberación de los detenidos en Guantánamo y su transferencia a los Estados Unidos también refleja de manera similar la influencia de las doctrinas y conceptos arraigados en la ley de inmigración. En la resolución Kiyemba v. Obama, el juez del distrito ordenó la liberación de los diecisiete prisioneros ugures de Guantánamo a los Estados Unidos, bajo los términos establecidos por la corte, después de determinar que su detención indefinida era ilegal. ${ }^{135} \mathrm{El}$ ejercicio de esa jurisdicción de hábeas, razonó la corte del distrito, tiene que incluir el poder para remediar el encarcelamiento ilegal, elaborando una orden de liberación apropiada. ${ }^{136}$ El circuito de D.C. revirtió la decisión. En un fallo dividido, el tribunal de apelaciones decidió en el caso Kiyemba v. Obama, que los jueces no podían ordenar la liberación de ningún detenido de Guantánamo a los Estados Unidos, incluso cuando no hubiera ningún remedio alternativo y el detenido como resultado permaneciera confinado en Guantánamo de manera indefinida. ${ }^{137}$

El Circuito de D.C. se basó en casos de inmigración para la proposición que hace que los partidos políticos tengan poderes plenos para excluir a individuos de los Estados Unidos. ${ }^{138}$ Bajo la resolución Kiyemba, el poder de los partidos políticos basado en inmigración para excluir el triunfo de una corte de distrito de conceder ayuda en un desafio basado en la Cláusula de Suspensión de hábeas corpus. ${ }^{139}$ El Circuito de D.C. hizo una analogía de la situación de los uigures con el caso de petición legal de Shaughnessy v. Los Estados Unidos Mezei, quien permaneció confinado en Ellis Island siguiendo su exclusión por razones de seguridad nacional

135. In re Guantánamo Bay Detainee Litigation [Litigio En $\operatorname{Re}$ Detenidos de la Bahía de Guantánamo], 581 F. Supp. 2d 33, 34 (D.D.C. 2008).

136. Id. en $42-43$.

137. Kiyemba v. Obama, 555 F.3d 1022 (D.C. Cir. 2009).

138. Kiyemba, 555 F.3d en 1025-26 (citando, inter alia, The Chinese Exclusion Case; Chae Chan Ping v. U.S. [El Caso de Exclusión China; Chae Chan Ping v. EE.UU.], 130 U.S. 581 (1889)).

139. HAFETZ, supra nota 12 , en 249 ("La corte de apelaciones se basó principalmente en el poder del gobierno federal para controlar la inmigración, a pesar de que los Uighurs estaban pidiendo sólo la liberación temporal de la prisión hasta que otro pais se pudiera encontrar para ellos y no solicitaron su ingreso a los Estados Unidos o estado legal bajo la ley de inmigración en EE.UU."). 
después que ningún otro país estuvo dispuesto a aceptarlo. ${ }^{140}$ Como en el caso de Mezei, la corte de apelaciones concluyó que los detenidos de Guantánamo no tienen ningún derecho a entrar en los Estados Unidos, temporalmente o de lo contrario, de no mediar autorización legislativa expresa, incluso si su exclusión resulta en su encarcelamiento indefinido, potencialmente permanente. ${ }^{141}$ Tal como lo ha interpretado el Circuito de D.C. en el caso Kiyemba, el poder de inmigración del gobierno federal corta abruptamente, si no niega potencialmente, el papel judicial reconocido en el caso de Boumediene. ${ }^{142}$ Un juez puede estar autorizado según la Cláusula de Suspensión de la constitución a ejercer la revisión de hábeas e invalidar el confinamiento de un solicitante, pero esto no puede anular las prerrogativas de los partidos políticos ordenando al solicitante en los Estados Unidos como una forma de mejoría. ${ }^{143}$ El fallo concurrente en el caso de Kiyemba se resistió a esta fusión de seguridad e inmigración, explicando que un juez de hábeas federal tenía el poder correctivo para ordenar a los prisioneros entrar a los Estados Unidos, pero que el tribunal de segunda instancia tenía que determinar primero si el gobierno tenía una base alternativa para detener a los solicitantes bajo la ley de inmigración antes de ordenar su liberación. ${ }^{144}$

La corte suprema tiene, sin duda, cierto escepticismo que ha expuesto sobre el enfoque del Circuito de D.C. La corte inicialmente concedió avocación en el caso Kiyemba, pero rehusó escuchar el caso por sus méritos después que el gobierno presentó nuevos hechos mostrando que había encontrado otros países donde los solicitantes podrían ser reubicados. ${ }^{145}$ Sobre la prisión preventiva, el circuito de D.C. sostuvo que estos nuevos hechos no modificaban su sentencia anterior, llegando a la conclusión de que el poder judicial no tenía poder para ordenar la liberación de un detenido de Guantánamo en los Estados Unidos bajo ninguna circunstancia,

140. Shaughnessy v. U.S., 345 U.S. 206, 207 (1953).

141. Kiyemba, 555 F.3d en 1028-29.

142. Ver Samuel Chow, The Kiyemba Paradox: Creating a Judicial Framework to Eradicate Indefinite, Unlawful Executive Detentions [La Paradoja de Kiyemba: Creación de un Marco Juridico Para la Erradicación de las Detenciones Ilegales Indefinidas], 19 CARDOzo J. INT'L \& COMP. L. 775, 797-98 (2011) (hablando sobre la desviación de la Corte en Kiyemba del papel del poder judicial reconocido en el caso Boumediene).

143. Id. (señalando la negación de la Corte de su propia capacidad para ordenar una liberación funcional).

144. Id. at 1032 (Rogers, J., concurring).

145. Kiyemba v. Obama, 130 S. Ct. 1235, 1235 (2010) (posición de la mayoría de los jueces) (revocando la decisión del Tribunal del distrito federal del circuito y devoldiendo el caso al Tribunal de apelación para que reconsidere su decisión a la luz de los nuevos hechos). 
por acción legislativa ausente expresa. ${ }^{146}$ Esta vez, la Corte suprema negó el pedido de avocación. ${ }^{147}$ Una declaración separada y firmada por cuatro jueces coincidiendo en la negación enfatizó la posibilidad continua de liberarlos en un tercer país. ${ }^{148}$ De esta manera, mientras la corte puede no compartir el punto de vista del Circuito de D.C. que un juez no pude pedir la liberación de un detenido de Guantánamo bajo ninguna circunstancia, considerando el poder de los partidos políticos en inmigración, es probablemente como ver el poder correctivo de un juez más limitado cuando existe otro país a donde el detenido puede ser transferido y la detención indefinida en Guantánamo no es la única alternativa. En cualquier caso, la decisión del Circuito de D.C. en Kiyemba sigue siendo la ley, y los jueces no tienen la autoridad para ordenar la liberación de un detenido de Guantánamo en los Estados Unidos incluso si no existe otra atenuante. ${ }^{149}$

\section{CONCLUSIÓN}

Casi una década después del 11/09, las políticas de seguridad nacional como la detención indefinida y los procesamientos militares a los sospechosos de terrorismo ya no parecen una aberración, pero se han convertido en una característica permanente del panorama jurídico. Parte de una "nueva normalidad" que ha sido adoptada por dos administraciones, apoyada por el congreso, y en gran medida sancionada por los tribunales. En esta amplia gama, en Estados Unidos el trato a los sospechosos de terrorismo tiene una gran similitud al trato dado a los inmigrantes y se basa en la aceptación de una alternativa de adjudicación enmarcada en juzgar los derechos de los no ciudadanos.

En lugar de disminuir con el tiempo, las prácticas asociadas con la "guerra contra el terror" amenazan con extenderse en unas nuevas maneras más radicales. Las recientes propuestas legislativas, por ejemplo, no se limitan a afirmar de manera expresa la autoridad del presidente para detener de manera indefinida a los no ciudadanos relacionados con el conflicto contra Al Qaeda, los talibanes y las fuerzas asociadas, lo cual AUMF hizo

146. Kiyemba v. Obama, 605 F.3d 1046 (D.C. Cir. 2010).

147. Kiyemba v. Obama, 131 S. Ct. 1631, 1631 (2011).

148. Id. en 1631 (declaración de Breyer, J., apoyado por Kennedy, Ginsburg, y Sotomayor, J.J., respetando la denegación de la petición de certiorari) (destacando que los solicitantes habían recibido con anterioridad ofertas para reasignarles (de las que al menos una se podria haber renovado); que no había prueba alguna de que la aceptación de su reasognamiento hubiese supuesto riesgo de tortura u otro maltrato para los prisioneros; y que el gobierno había continuado la búsqueda de nuevas opciones de reaignamiento). La Juez Kagan se obstuvo debido a su papel previo como Procuradora General.

149. Kiyemba, 555 F.3d at 1026-29. 
sólo por implicación. ${ }^{150}$ Las propuestas también exigen la detención militar de los no ciudadanos sospechosos de terrorismo, sujetos a una renuncia por el secretario de defensa que la detención militar no era parte de los intereses de la seguridad nacional. Por el contrario, las propuestas excluyen a los ciudadanos de la detención militar obligatoria. ${ }^{151}$ Tales medidas no sólo ilustran como las políticas subyacentes a Guantánamo se están ampliando internamente mientras se van institucionalizando. Las propuestas legislativas también sugieren como las políticas de contraterrorismo que extienden la autoridad del gobierno para la detención, restringen los derechos de los no ciudadanos, y crean un sistema de justicia a dos niveles que se asemejan al enfoque existente en los Estados Unidos desde hace mucho tiempo con respecto a los inmigrantes en sentido general.

150. Ver S. 1253, 112th Cong., 1st Sess., $§ 1031$ (2011) [en lo sucesivo S. 1253]. Las propuestas proveen una autoridad más limitada a la hora de detener ciudadanos, así como a residentes permanentes legales en el mismo conflicto. Id. § 1031(d) (estableciendo que la autoridad de detención militar bajo el estatuto "no se extiende a la detención de ciudadanos o extranjeros que sean residentes legales de los Estados Unidos, basándose en que la conducta tuvo lugar en los Estados Unidos excepto en lo permitido por la Constitución de los Estados Unidos").

151. Id. $\S 1032(\mathrm{a})$. 



\title{
PLANIFICACIÓN URBANÍSTICA Y CATÁSTROFES NATURALES UNA PERSPECTIVA EUROPEA DESDE ESPAÑA
}

\author{
Dr. Julio Ponce*
}

I. INTRODUCCIÓN

A. Catástrofes Naturales Y Legislación Urbanística:

Una Posible Causa, Pero También Un Posible

Mecanismo Para Prevenirlas.

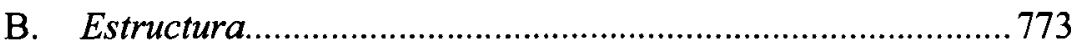

II. UNIÓN EUROPEA Y ESTADOS MIEMBROS: DISTRIBUCIÓN DE

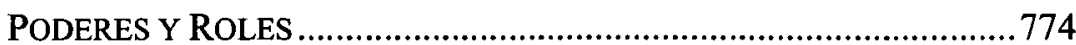

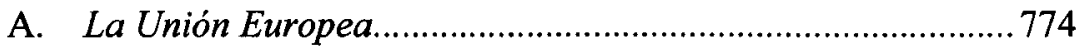

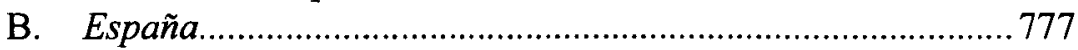

1. El Marco del Derecho Urbanístico Español .....................779

2. La Perspectiva Histórica................................................779

a. La Situación antes de 1956.........................................779

b. Ley Nacional de 1956. La Importancia de la

Planificación en España ..........................................780

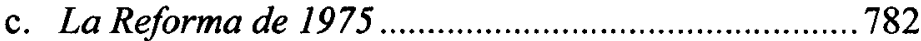

d. La Constitución de 1978............................................782

3. La Distribución del Poder Entre Niveles Públicos.

Legislación Regional.....................................................783

4. La Fragmentación de las Leyes del Uso del Suelo y la

Supervivencia de un Núcleo Duro Nacional .....................784

III. EVALUACIÓN Y GESTIÓN DE LOS RIESGOS POR LA LEY DEL

USO DEL SUELO: El PROBLEMA ESPECÍFICO DE INCERTIDUMBRE

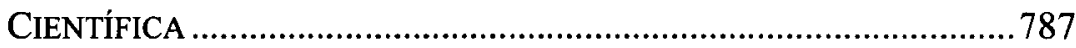

A. Incertidumbre Cientifica y el Principio de Precaución ...........787

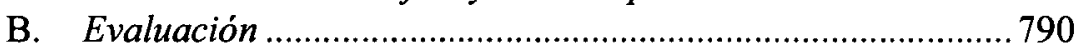

1. Riesgos Naturales: Las Directivas de la U.E. y Su Impacto a Nivel Nacional: el Caso Español.

2. Riesgos Naturales: los requisitos legales de evaluación según la planificación: Evaluación del Impacto Ambiental y legislación del suelo Española

* Me gustaría agradecer al Sr. Oscar Capdeferro, estudiante de doctorado en la Facultad de Derecho, su ayuda en la búsqueda y organización de los datos jurídicos. Este estudio fue debatido durante el Taller sobre "Desastres y Estudios Socio Juridios," presidido por Susan Sterett (Universidad de Denver) y Thomas Birkland (North Carolina State University), que se celebró en el Instituto de Oñati Internacional para la Sociologia del Derecho (http://www.iisj.net/sesion=1347) el jueves 21 de julio de 2011 . 
a. Evaluación del Impacto Ambiental y legislación nacional del suelo Española.

b. Prevención de riesgos de inundación y planificación urbana.

3. Riesgos Creados Por el Hombre: el Ejemplo de la

Evaluación de los Riesgos de Crimen y Terrorismo

C. Gestión

1. Prohibiciones Legales Vinculantes para la

Planificación Urbanística

2. Prohibiciones Legales Vinculantes Para La

Planificación Urbanística Y los Propietarios Privados:

La Función Social De La Propiedad Y La Prevención

De Catástrofes Naturales

a. Planificación Regional y Zonificación Local ............. 793

b. Limites al Derecho de Propiedad y las

Catástrofes Naturales.

IV. PlanifiCAR Y ReClamar DaÑos Y PERJUICIOS EN CASO DE DESASTRES NATURALES 794

A. Falta de Prevención: Responsabilidad Pública Y Privada.... 794

B. Algunos ejemplos españoles acerca de las reclamaciones de indemnización en caso de catástrofes

1. El camping de Biescas.

2. El Atentado de un Coche Bomba contra un Cuartel de la Guardia Civil

V. LA ORDENACIÓN DEL TERRITORIO Y LA PREVENCIÓN DE

DESASTRES

A. Ubicación de las Centrales Nucleares y Prevención de Riesgos a Través de la Planificación Urbana: Los Procedimientos Administrativos y los Derechos

Fundamentales. 798

B. La Planificación y la Prevención de los Riesgos de Terrorismo. 800

\section{INTRODUCCIÓN}

A. Catástrofes Naturales Y Legislación Urbanistica: Una Posible Causa, Pero También Un Posible Mecanismo Para Prevenirlas

Una de las principales preocupaciones de Europa son las catástrofes naturales y las provocadas por la acción humana. Ello es debido al aumento de su frecuencia y gravedad, y al impacto sobre la vida humana, la destrucción de infraestructuras sociales y económicas y el daño que causan en el medio ambiente. Por ejemplo, de acuerdo con un documento oficial 
de la Unión Europea (U.E.), el impacto económico de las catástrofes naturales en Europa ha sido estimado en $€ 15$ billones anuales. ${ }^{1}$

La legislación urbanística (Derecho urbanistico, en España, o Droit de l'urbanisme, en Francia) regula el uso del suelo. La relación entre el uso del suelo y las catástrofes naturales es bastante clara; por una parte la regulación del suelo permite la expansión urbana a través de varios instrumentos (planificación, zonificación), y la expansión urbana lleva consigo un intenso uso del suelo, el desarrollo industrial y la construcción de infraestructuras que pueden llegar a ser un factor decisivo de posibles catástrofes naturales en un futuro. Un buen ejemplo es el efecto que tiene la presión urbana sobre los ríos y los consecuentes riesgos de inundaciones. $^{2}$

Pero por otra parte, la regulación sobre el uso del suelo puede ser también un buen instrumento de prevención de estas catástrofes, previniendo que ocurran y minimizando sus impactos cuando su ocurrencia es inevitable. Esta es la perspectiva que pretendo estudiar en este artículo.

\section{B. Estructura}

Mi estudio estará organizado de la siguiente manera: en primer lugar, consideraré el papel de la U.E. y de sus Estados Miembros en relación con las catástrofes naturales y la ordenación del territorio.

En segundo lugar, abordaré la cuestión de cómo la ordenación del suelo puede evaluar y gestionar los riesgos para evitar catástrofes naturales, prestando especial atención al uso del principio de precaución europeo, también conocido como "Más vale prevenir que curar." En este análisis utilizaré especialmente como ejemplo, aunque no exclusivamente, el sistema legal Español, teniendo en cuenta su inclusión en el sistema general de la Unión Europea.

En tercer lugar, reflexionaré sobre la responsabilidad pública de prevenir catástrofes naturales y las posibles consecuencias de una mala administración a la hora de tomar decisiones sobre el uso del suelo. Para ello tomaré como referencia dos casos españoles; la catástrofe de Biescas y

1. Comunicación de la Comisión al Parlamento Europeo, al Consejo, al Comité Económico y Social Europeo y al Comité de las Regiones, Un Enfoque Comunitario para la Prevención de Catástrofes Naturales y de Origen Humano, en 4, n.11, COM (2009) 82 final (23 de febrero del 2009), disponible en http://europa.eu/legislation_summaries/environment/civil_protection/pr0005_es.htm (última visita el 31 de octubre de 2011) [en lo sucesivo Prevención de Catástrofes Naturales y de Origen Humano].

2. Ver, por ejemplo, MINISTERIO DE MEDIo AMBIENTE Y MEDio RuRAL Y MARINO, RESTAURACIÓN DE Ríos: BASES dE LA ESTRATEgIa NaCIONAL DE RESTAURACIÓN DE RÍos 47 (abril del 2010), disponible en http://www.marm.es/es/agua/publicaciones/Rios_B_Restauracion_tcm727570.pdf (última visita el 31 de octubre del 2011) (Esp.). 
un ataque terrorista contra la policia española. ${ }^{3}$ En ambos casos se interpusieron acciones legales contra las autoridades públicas por parte de las personas afectadas buscando una compensación por los daños sufridos debido a una inapropiada planificación. ${ }^{4}$

Finalmente, analizaré las posibilidades de la planificación urbanística como instrumento para prevenir catástrofes, prestando especial atención a dos temas específicos: la ubicación de centrales nucleares (con reflexiones sobre la importancia de procedimientos administrativos que protejan los derechos fundamentales, tal y como subrayó el Tribunal Constitucional Alemán en su decisión Mülheim-Karlich.) $)^{5}$ y los nuevos desarrollos sobre la prevención de la delincuencia y de los ataques terroristas por medio de la planificación urbanística (conocidos como Prevención del Crimen Mediante el Diseño Ambiental).

\section{UNIÓN EUROPEA Y ESTADOS MIEMBROS: DISTRIBUCIÓN DE PODERES Y ROLES}

Aunque la U.E. no tiene poderes en el ámbito de la planificación del suelo, sí puede ejercer influencia en la política nacional de diferentes maneras. Además, aunque los Estados Miembros sean los responsables de la planificación del suelo, también hay que tener en cuenta que están vinculados por el marco jurídico de la U.E. y por su propia distribución constitucional de competencias. Utilizaré como ejemplo el caso español.

\section{A. La Unión Europea}

El territorio actual de la U.E. ocupa 4,324,782 $\mathrm{km}^{2}$ (menos de la mitad del tamaño de los EE.UU). ${ }^{6}$ La U.E. tiene una población de 492,387,344 (Julio 2010 est.) ${ }^{7}$ y una densidad de 112 habitantes $/ \mathrm{Km}^{2}$ (comparado con una densidad de 31 en EE.UU.). ${ }^{8}$ El $75 \%$ de los europeos vive en áreas

3. Ver en general A.N., 21 de diciembre del 2005 (R.O.J., No. 1976), disponible en $\mathrm{http}: / \mathrm{www}$.poderjudicial.es/search/doAction?action=contentpdf\&databasematch=AN\&reference $=1022$ 464\&links=\&optimize=20060118 (última visita el 21 del octubre de 2011) (Esp.).

4. Id.

5. Ver Bundesverfassungsgericht [BVerfG] [Tribunal Federal Constitucional], 20 de diciembre de 1979, ENTSCHEIDUNGEN DES BUNDESVERWALTUNGSGERICHTS [BVERFGE] 53, 30 (1BvR 385/77) (Alem.).

6. Ver CIA, World Fact Book [CIA, Libro de los Hechos], https:/www.cia.gov/library/publications/the-world-factbook/index.html (última visita el 31 de octubre del 2011) [en lo sucesivo Libro de los Hechos].

7. Id.

8. División de Población del Departamento de Asuntos Económicos y Sociales de la Secretaría de las Naciones Unidas, Previsiones Demográficas Mundiales: Revisión de 2006 y Perspectivas de Urbanización en el Mundo (revisión del 2005), 
urbanas y se estima que para 2020 el porcentaje se elevará al $80 \% .^{9}$ El fenómeno de la urbanización se está extendiendo rápidamente, más que el crecimiento de la población urbana. Se estima que el tamaño total de las áreas construidas ha crecido en una quinta parte en los últimos veinte años, mientras que la población en la U.E. ha crecido solo un $6 \% .{ }^{10}$

El modelo Europeo ha sido un concepto muy discutido durante los debates a cerca de la integración Europea. ¿Pero cuál es su significado? Obviamente, es un concepto con claras raíces políticas, en desarrollo y en debate continuo." Algunas opiniones conciben la U.E. como un área de libre comercio, atribuyendo el pobre rendimiento de la economía al modelo "débil" Europeo que se invoca. A estas personas les gustaría ver el papel de las instituciones europeas limitado a controlar el Mercado Único. ${ }^{12}$ Por otro lado, otras opiniones consideran esa perspectiva cercana al concepto de laissez-faire del liberalismo Anglo-Sajón. Estas opiniones ven al modelo europeo como las bases de una sociedad justa y competitiva, deseando instituciones Europeas fuertes que lleven a cabo funciones que de otra forma estarian reservadas a las instituciones nacionales de los propios Estados. ${ }^{13}$ Esta segunda corriente de pensamiento concibe el modelo Europeo como un orden humano basado en una economía mixta, relaciones laborales civilizadas, estado del bienestar y un compromiso con la justicia social. $^{14}$

Los académicos y profesionales que discuten sobre la planificación espacial y la política de cohesión territorial en la U.E. también tienen en cuenta este debate. En cualquier caso, el marco legal Europeo (interpretado en relación a un gran número de documentos oficiales en línea con los desarrollos internacionales) considera la sostenibilidad un concepto clave compuesto por tres partes complementarias: la económica, la social y la

http://esa.un.org/wpp/unpp/panel_population.htm (última visita el 31 de octubre del 2011) (seleccionar "population", después seleccionar "Europe", después seleccionar "population density", después añadir "United States", y después pulsar "display").

9. Memorándum de Buero de los Asesores de Política Europea, Roger Liddle \& Frederic Lerais, Europe's Social Reality [Realidad Social Europea], MEMO/07/83 (26 de febrero del 2007), disponible en http://europa.eu/rapid/pressReleasesAction.do?reference=MEMO/07/83 (última visita el 31 de octubre de 2011 ).

10. Id.

11. Ver por todos Andreas Faludi, Territorial Cohesion and the European Model of Society [Cohesión Territorial y El Modelo Europeo de Sociedad], at 2 (julio del 2005) (articulo no publicado presentado en AESOP Viena en el 2005), disponible en http://aesop2005.scix.net/data/papers/att/228. fullTextPrint.pdf (última visita el 31 de octubre del 2011).

12. Id.

13. Id.

14. Id. (considera que la segunda perspectiva de un modelo arraigado en social-demócratacristiano corriente principal demócrata de la política continental europea). 
medioambiental. ${ }^{15}$ En otras palabras, no puede decirse que una sociedad sea sostenible a menos que estos tres elementos estén presentes.

El Libro Mundial de Hechos de la CIA establece que "la evolución de la Unión Europea (EU) desde el acuerdo regional económico de 6 estados vecinos en el año 1951 hasta la actual organización supranacional de 27 países de todo el continente Europeo, es un fenómeno sin precedente en los anales de la historia." ${ }^{\text {"16 }}$ Bien, en cualquier caso deberíamos considerar esta unificación como un proceso en marcha y difícil. La aventura empezó en 1957 y continúa hoy en día con una constitución fallida en 2004 y el último Tratado de Lisboa que ha cambiado el marco legal existente. ${ }^{17}$

Es importante subrayar que, de acuerdo con este marco constitucional, la U.E. no tiene poderes en el ámbito del uso del suelo. ${ }^{18}$ De todas formas, como el Profesor Italiano Chiti ha destacado, los objetivos del desarrollo sostenible, la solidaridad, la lucha contra la exclusión y la cohesión económica, territorial y social llevan a un aumento de la intervención de la U.E. en relación con las ciudades Europeas. ${ }^{19}$ En lo que sí tiene competencia la U.E. es en los campos relacionados con el medio ambiente y otras políticas de interés público que tengan un impacto territorial (por ejemplo, transporte), eso sí, teniendo en cuenta el principio de subsidiariedad (es decir, que los asuntos deben ser tratados por la autoridad competente más próxima al objeto del problema). ${ }^{20}$

Como se puede ver, la U.E. ejerce un significativo papel en los asuntos urbanos a través de estos poderes relacionados entre sí, la voluntad política de tratar los problemas territoriales mediante la promoción de contactos con redes intelectuales y públicas (generando un gran número de informes, estudios y papeles) y la realización de importantes inversiones económicas. Además, la política de medio ambiente de la U.E. tiene un impacto significativo en la legislación urbanística de los países Europeos. ${ }^{21}$

15. Ver, por ejemplo, The Urban Dimension in European Union Policies for the Period 20072013 [Dimensión Urbana de las Políticas de la Unión Europea Para el Periodo 2007-2013] (2010), http://ec.europa.eu/regional_policy/sources/docgener/guides/urban/index_en.htm (última visita el 31 de octubre del 2011).

16. Libro de los Hechos, supra nota 6.

17. Ver Tratado de Lisboa por el que se Modifican el Tratado de la Unión Europea y el Tratado Constitutivo de la Comunidad Europea, el 13 de diciembre del 2007, 2007 O.J. (C 306) 1, 26971 [en lo sucesivo Tratado de Lisboa].

18. Id.

19. ERMINIO FERRARI ET AL., IL GOVERNO DEL TERRITORIO [El GOBIERNO DEL TERRITORIO] (A. Giuffrè ed., 2003).

20. Id.

21. Al lado de las principales fuentes de Derecho de la U.E., hay que tener en cuenta las fuentes secundarias, es decir previsiones legislativas que se toman y aplican en referencia a un artículo del Tratado CE. Hay diferentes tipos de legislación secundaria, como veremos más adelante. 


\section{B. España}

En 2007 España tenía una población de 45,200,737 con un área de $506,030 \mathrm{~km}^{2}$ y una densidad de 89 habitantes $/ \mathrm{km}^{2}$, situándolo en el medio del ranking Europeo entre la población más densa de los países de Europa central y los menos poblados de los países del norte. ${ }^{22}$ España ha visto crecer su población rápidamente, especialmente desde 1960-1970 y 19701980. Ello ha sido promovido por la creciente industrialización que se experimenta en áreas metropolitanas de las grandes ciudades como Madrid, Barcelona, Valencia, Bilbao y Zaragoza. ${ }^{23}$ Desde 1960-1970 todas estas áreas urbanas tuvieron un crecimiento anual de la población de más del $3 \%$, $\mathrm{y}$ en el caso de Madrid más de un 4\%. ${ }^{24}$ El periodo de 1970-1980 también experimentó un rápido crecimiento de la población aunque a un porcentaje más bajo, $2 \%$ en Madrid o incluso menos. ${ }^{25} \mathrm{El} \mathrm{crecimiento} \mathrm{de} \mathrm{la} \mathrm{población}$ se redujo considerablemente en las décadas consecutivas, tocando fondo al final del periodo de 1990-2000. Desde el año 2000 en particular, la constante inmigración ha contribuido a un aumento estable de la tasa natural de crecimiento. ${ }^{26}$

El rápido crecimiento de la población desde 1960 a 1980 , concentrado en las áreas metropolitanas de las grandes ciudades, ha producido un déficit importante en infraestructuras, viviendas y servicios, y un consecuente deterioro de la calidad de vida urbana. ${ }^{27}$ Además, desde mediados de los años 70 también se ha producido un declive industrial en lugares como Bilbao o el área central de Asturias, sedes del hierro y del acero y del sector del transporte maritimo que entró en crisis en toda Europa Occidental. ${ }^{28} \mathrm{La}$ tasa de crecimiento respecto al desarrollo de las viviendas en los últimos años ha sido espectacular y de hecho se mantuvo hasta la crisis del 2008. Ello fue acompañado de un fuerte aumento de los precios de las viviendas, haciendo que comprar una casa fuera muy difícil para gran parte de la población. Pero actualmente, la caída del tamaño promedio de las viviendas y el aumento constante de la inmigración han generado una nueva demanda de viviendas. La población urbana de España está concentrada en 4 áreas principales, cada una con más de 1,000,000 habitantes (Madrid, Barcelona, Valencia y Sevilla). Estas áreas están situadas, a excepción de

22. Urban Policies of Spain [Políticas Urbanas en España], http:/www.eukn.org/Spain/en/EUKN_Spain/Urban_Policies_in_Spain (última visita el 31 de octubre del 2011) [en lo sucesivo Políticas Urbanas en España].

23. Id.

24. Id.

25. Id.

26. Id

27. Políticas Urbanas en España, supra nota 22.

28. Id. 
Madrid, en la periferia de la península; nueve áreas urbanas de entre 500,000 y 1,000,000 habitantes (Bilbao, Málaga, el área central de Asturias, Zaragoza, Alicante/Elche, la Bahía de Cádiz, Vigo/Pontevedra, Murcia y Las Palmas de Gran Canaria); treinta y cinco áreas urbanas de entre 100,000 y 500,000 ; y treinta de entre 50,000 y $100,000{ }^{29}$ Es decir, hay setenta y ocho áreas urbanas distribuidas por toda España con más de 50,000 habitantes. $^{30}$ Las características fisicas, las comunicaciones, la ubicación de enclaves industriales y los asentamientos turísticos costeros demuestran que la población está distribuida de manera desigual; concentrada particularmente en la periferia de la península y el área metropolitana de Madrid (zona central del país caracterizada por una baja densidad de población). ${ }^{31}$ No sólo el área metropolitana de Madrid está experimentando una alta tasa de crecimiento, sino que poblaciones más pequeñas como Málaga, Alicante/Elche, y en particular Murcia y Vigo/Pontevedra, también están creciendo rápidamente. ${ }^{32}$ La tendencia de crecimiento permanece constante en las regiones situadas en la periferia de la península, especialmente las zonas turísticas de la costa Mediterránea, y en el área central de Madrid. ${ }^{33}$

España ha experimentado un crecimiento natural bastante bajo desde 1990 hasta el año 2000, y tiene una de las tasas de fertilidad más bajas del mundo, muy por debajo de la media Europea. ${ }^{34}$ En 1996 la media de niños por mujer era de 1.16 frente a 1.44 en el resto de la U.E. ${ }^{35}$ El continuo crecimiento de inmigración desde el año 2000 ha causado un ascenso de este indicador, pero aun así permanece por debajo de la media Europea. El envejecimiento de la población supone muchos problemas, como la provisión de pensiones de jubilación, la necesaria prestación de asistencia sanitaria y una mayor demanda de infraestructuras para los ciudadanos mayores. $^{36}$

El crecimiento de la inmigración produce diversos efectos: por una parte, el rejuvenecimiento de la población, más trabajadores jóvenes en el mercado y consecuentemente más contribuciones al sistema de la seguridad social; mientras que por otra parte aumenta la demanda de infraestructuras $\mathrm{y}$ viviendas, y causa problemas en el ámbito de la integración social. ${ }^{37}$

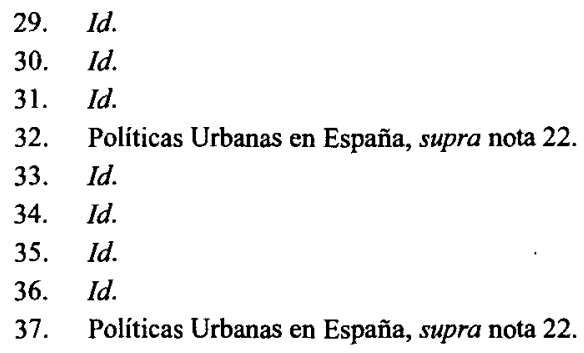




\section{El Marco del Derecho Urbanístico Español}

En esta sección me centraré en las tendencias modernas referentes al Derecho urbanístico histórico y actual, comparando la situación en España con la situación en América y otras legislaciones urbanísticas Europeas. Obviamente la jurisprudencia es muy importante, pero la posición más relevante la ocupa la legislación, la cual ha creado el Derecho urbanístico moderno en España, con la introducción de varias Leyes bastante técnicas.

Por otra parte, la planificación urbanística tiene un mayor rol en este escenario. Ésta es obligatoria a nivel regional (decisiones tomadas por las Comunidades Autónomas), y más importante aún, a nivel local. En España, la planificación urbanística conlleva diferentes elementos legales: varios tipos de mapas, algunos documentos, y las normas de división del suelo en diferentes zonas (clasificación y calificación). El uso del suelo debe estar regulado de acuerdo con un plan, requisito legal común en todas las Comunidades Autónomas (actualmente hay diecisiete Comunidades Autónomas en España). ${ }^{38}$

\section{La Perspectiva Histórica}

\section{a. La Situación antes de 1956}

La regulación del uso del suelo ha existido en España desde los tiempos del Ancièn Régime. ${ }^{39}$ El contexto moderno tiene su origen en el estado liberal del siglo XIX, bajo la jurisdicción de la policía a nivel local. ${ }^{40}$ La regulación se centraba en el crecimiento de las ciudades (por ejemplo, el desarrollo urbano del ensanche) y en problemas de salud y seguridad. ${ }^{41} \mathrm{La}$ intervención pública fue posible por medio de un amplio número de leyes (por ejemplo, ordenanzas, alineaciones y expropiación, la cual fue regulada por primera vez en 1836 por la Ley de Expropiación). ${ }^{42}$

El desarrollo de la legislación del suelo tuvo un carácter más técnico durante el siglo XX. Desde 1920, técnicas legales más modernas fueron incluidas en los códigos, como por ejemplo, la Carta Municipal de 1924 que

38. Juli Ponce, Land Use Law, Liberalization, and Social Cohesion through Affordable Housing in Europe: The Spanish Case [Derecho de Uso de la Tierra, la Liberalización y la Cohesión Social Mediante la Vivienda en Europa: el Caso Español], 36 URB. L. 317, 322-31 (2004).

39. Ver Allan-RandolPh BREWER-Carias, La CIUdad ORdenada (Criteris Editorial 2006) ( $y$ bajo el dominio colonial español una regulación similar de usos de la tierra entró en vigencia en América del Sur).

40. Ponce, supra nota 38 , en 322.

41. Id. en 323.

42. Martín Bassols Coma, GÉnesis y Evolución del Derecho Urbanistico EsPañol (1812-1956) (Montecorvo, 1973). 
incluyó la técnica de la zonificación. ${ }^{43}$ En los años 30 llega a España la idea de la Planificación Regional. Cataluña fue la comunidad pionera con el "Plan de Distribución en Zonas" en 1932, pero la Guerra Civil Española destrozó la posibilidad de desarrollos más concretos. ${ }^{44}$

Se desarrolló un proyecto de ley en relación con las viviendas asequibles en 1878 y se crearon varios comités de investigación la ley de Casas Baratas de 1911 fue la primera ley sobre viviendas para las clases trabajadoras. ${ }^{45}$ La ley se basaba en inversiones urbanas y concedía algunas subvenciones públicas para empresarios. ${ }^{46}$ Desafortunadamente, esta legislación no tuvo éxito, principalmente porque no había suficientes recursos públicos para desarrollar las disposiciones, y fue emendada antes del principio de la Guerra Civil en $1936{ }^{47}$

Después de la Guerra Civil, muchos esfuerzos se dirigieron a reconstruir el devastado país. Con este propósito se creó el Instituto Nacional de Vivienda, una institución pública creada en el año $1939 .{ }^{48}$ Algunos años después, en 1957, el Ministerio de la Vivienda asumió la responsabilidad de la política de la vivienda en España hasta que se integró en el Departamento de Trabajo Público a finales de los años $70 .^{49}$ En 2004, el Ministerio de Vivienda fue restaurado para tratar el problema de la vivienda asequible. ${ }^{50}$ Aunque a finales de 2010 , después de una nueva reestructuración política, este Ministerio volvió a desaparecer. ${ }^{51}$

\section{b. Ley Nacional de 1956. La Importancia de la Planificación en España}

El Derecho Urbanístico moderno fue introducido en 1956, durante los cuarenta años de dictadura de Franco. ${ }^{52}$ La Ley de 1956 entró en vigor

43. Ver Estatuto Municipal (B.O.E. 1924, 69), disponible en http://www.boe.es./ datos/pdfs/BOE/1924/069/A01218-01302.pdf (última visita el 31 de octubre del 2011) (Esp.).

44. Ver Carlos Nardiz Ortiz, Los Ingenieros de Caminos y el Urbanismo del Siglo XX, 59 FIGURE 3 (1999), disponible en http:/www.ciccp.es/revistait/Anteriores/pdf/Op49/ OP49\%20Articulos/06.Carlos\%20Nardiz\%200rtiz.pdf (última visita el 31 de octubre del 2011) (Esp.).

45. Juli Ponce Solé et al., El DeREcho de la VIVIENDa en El Siglo XXI: Sus RELACIONES CON LA ORDENACIÓN DEL TERRITORIO Y EL URBANISMO. CON ANÁLISIS ESPECÍFICO DE la ley Catalana 18/2007, de 28 Del Diciembre, en su Contexto EsPañol, EuRopeo E INTERNACIONAL (Marcial Pons 2008) ("Breve reseña histórica de la regulación de la vivienda en España y Cataluña existente con anterioridad a la Ley 18/2007, de 28 del diciembre, del derecho a la vivienda.") (Esp.).

46. Id.

47. Id.

48. Ponce, supra nota 38 , en 323.

49. Id.

50. Id.

51. Id.

52. TOMÁs-RAMÓN FERNÁNDEZ, MANUAL DE DERECHO URBANístico 23 (Las Rozas, Madrid El Consultor de los Ayuntamientos y de los Juzgados, 2008). 
cuando España era un país centralizado y no democrático. ${ }^{53}$ A pesar de esta situación, los académicos están de acuerdo en que esta ley fue de una alta calidad técnica y la base del Derecho moderno sobre el uso del suelo en España. ${ }^{54}$

El punto de partida de la regulación tenía como prioridad las tierras agrícolas. ${ }^{55}$ Cualesquiera construcciones posteriores se entendía que eran concedidas por poderes públicos a través de la planificación urbanística. ${ }^{56}$ Como norma general, los planes regulaban el derecho a la propiedad, sin expropiación. ${ }^{57} \mathrm{Y}$ el plan (al menos formalmente) otorgaba el poder de la toma de decisiones a las municipalidades, con un elemento de interpretación discrecional. ${ }^{58}$ Sin embargo, esta dirección no era ejercida por municipalidades demócratas y había regularmente abusos de poder a favor de los partidarios de los alcaldes de la dictadura franquista. Consecuentemente, la planificación urbana fue la principal pièce de résistance de todo el sistema legal. Pero veinte años después de la ley de 1956, solo el 7.5\% del territorio Español tenía un plan urbano implementado. ${ }^{59}$ De tal manera que el fracaso del plan fue en realidad un fracaso de la ley.

Por otro lado, con respecto a la gestión del desarrollo, el Derecho español no conoce de exacciones del tipo americano, ni de cuotas de impacto, ni de impuestos franceses sobre el uso del suelo como una forma de financiación para los nuevos desarrollos urbanos de las ciudades. La ley de 1956 estableció un sistema legal para desarrollar las infraestructuras públicas y los equipamientos públicos, el cual sigue funcionando, basado grosso modo en los deberes legales del propietario de ceder gratuitamente un porcentaje de terreno, fijado legalmente, al Ayuntamiento. Esto sigue siendo efectivo hasta el día de hoy en términos generales, con un máximo nacional legal y un posible mínimo variable en cada Comunidad Autónoma, de cesión del terreno necesario para las calles, áreas verdes $\mathrm{e}$ infraestructuras públicas locales y de hacer todos los trabajos necesarios para desarrollar el área que incluye la parcela de suelo cedida. ${ }^{60}$

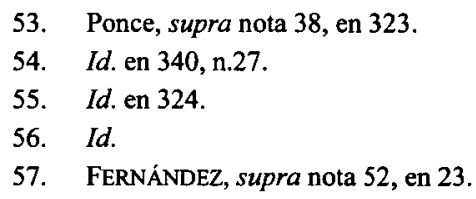

53. Ponce, supra nota 38, en 323.

54. Id. en 340, n. 27 .

55. Id. en 324 .

56. Id.

57. FERNÁNDEZ, supra nota 52, en 23.

58. Id. (Aunque las políticas de las ciudades estaban en manos de los entes locales, la realidad es que el nivel nacional interferido constantemente, cambiando las decisiones locales. Tenemos que tener en cuenta que antes de la Constitución de 1978 no existía la idea de la autonomía local, como a nivel nacional tuvo la "custodia" de las autoridades locales.).

59. Ponce, supra nota 38, en 324.

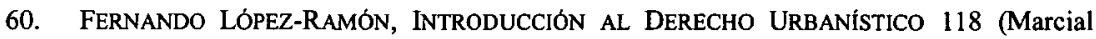


Pero en el mundo real este rígido sistema se hace más flexible por medio de acuerdos de desarrollo (convenios urbanísticos) entre los Ayuntamientos y el promotor, lo que en algunos casos supone una vía de entrada de la corrupción. Los acuerdos de desarrollo entre las municipalidades y los dueños o promotores son regulados en las leyes urbanísticas. Éstos son válidos en el sistema legal español, pero están sometidos a ciertas condiciones procesales con el fin de promover la transparencia.

\section{c. La Reforma de 1975}

Durante los años 1960 y parte de 1970, España se volvió un país industrializado, sufriendo grandes transformaciones sociales y estructurales. En el ámbito urbano, el fenómeno clave fue la emigración de una gran parte de la población de los sectores agrícolas a las ciudades, con los consecuentes problemas relativos a las viviendas asequibles. $\mathrm{El}$ crecimiento de las ciudades fue absolutamente caótico. Teóricamente, los planes estaban en marcha para hacer frente a esta emigración, pero un gran número de los municipios no aprobaron los planes y en otros casos, como mencioné anteriormente, las decisiones arbitrarias dieron raíz a especulaciones e hicieron imposible obtener una expansión urbana ordenada.

Debido a estos factores, se decidió introducir una segunda Ley en 1975, para completar la de 1956 y evitar todos esos problemas. ${ }^{61}$ Pero la estructura general del sistema legal permaneció intacta. En 1976, las leyes de 1956 y 1975 se unificaron en una sóla (el Texto Refundido). ${ }^{62}$

\section{d. La Constitución de 1978}

La Constitución de 1978 reflejó los profundos cambios en España, con la introducción de la democracia y de la autonomía de las regiones (pasamos, efectivamente, de un modelo centralizado a uno casi federal). Ambos elementos tuvieron un impacto legal en esta materia, tanto a nivel autonómico $^{63}$ como a nivel local. ${ }^{64}$ Una ley de 1985 menciona

61. Reforma de la Ley Sobre Régimen del Suelo y Ordenación Urbana, (B.O.E. 1975, 107), disponible en http://www.boe.es/boe/dias/1975/05/05/pdfs/A09427-09448.pdf (última visita el 31 de octubre del 2011) (Esp.).

62. LÓPEZ-RAMÓN, supra nota 60, en 118.

63. CONSTITUCión ESPAÑola, B.O.E. n.148.1.3, el 29 de diciembre del 1978, disponible en http:/www.congreso.es/portal/page/portal/Congreso/Congreso/Hist_Normas/Norm/const_espa_texto_in gles_0.pdf (última visita 31 de octubre del 2011) (Esp.) [en lo sucesivo CONSTITUCión ESPAÑOLA].

64. Id. n.140. La Constitución garantiza la autonomía de los municipios. Estos gozarán de personalidad jurídica plena. Su gobierno y administración corresponde a su ciudad en los Consejos, 
específicamente las regulaciones del uso del suelo como una de las competencias de las autoridades locales. ${ }^{65}$

De acuerdo con la Constitución, España fue declarada un "Estado Social, ${ }^{, 66}$ y varios derechos sociales fueron introducidos incluyendo el derecho al medio ambiente. El artículo 45 establece: ${ }^{67}$

1) Todos tienen el derecho a disfrutar el medio ambiente adecuado para el desarrollo de la persona, así como el deber de conservarlo.

2) Los poderes públicos velaran por la utilización racional de todos los recursos naturales, con el fin de proteger y mejorar la calidad de la vida y defender y restaurar el medio ambiente, apoyándose en la indispensable solidaridad colectiva.

3) Para quienes violen lo dispuesto en el apartado anterior, en los términos que la ley fije se establecerán sanciones penales $o$, en su caso, administrativas, así como la obligación de reparar el daño causado.

Ese sistema legal demuestra un nivel alto de complejidad que tiene que ser administrado a través de diferentes mecanismos legales de cooperación y coordinación interadministrativa. ${ }^{68}$

\section{La Distribución del Poder Entre Niveles Públicos. Legislación Regional}

Haciendo uso de la Cláusula Constitucional del artículo 148.1.3, las diecisiete Comunidades Autónomas (y entre ellas Cataluña, cuya capital es la ciudad de Barcelona, con una larga historia, su propio idioma y una fuerte identidad de su condición de nación) han promulgado diversas leyes, creando su propio Derecho sobre la vivienda y el uso del suelo. ${ }^{69}$ Aunque el gobierno central ha delegado muchos de sus poderes, ha continuado promulgando leyes sobre el uso del suelo usando varias cláusulas constitucionales (especialmente el art.149.13, el cual le permite promulgar

integrados por los Alcaldes y concejales. Concejales serán elegidos por los vecinos del municipio mediante sufragio universal, igual, el sufragio libre, directo y secreto, en la forma prevista por la ley. Los Alcaldes serán elegidos por los concejales o por los vecinos. La ley establecerá los términos en que un cabildo abiertode todos los residentes pueden continuar.

65. Ver Ley Reguladora de las Bases del Régimen Local tit. 8 (B.O.E. 1985, 7) (Esp.).

66. CONSTITUCIÓN ESPAÑOLA, supra nota 63, art. 45.

67. Id.

68. Ver Modificación de la Ley de Régimen Jurídico de las Administraciones Públicas y del Procedimiento Administrativo Común (B.O.E. 1992, 285), disponible en http://www.boe.es/boe/dias/1992/11/27/pdfs/A40300-40319.pdf (última visita el 31 de octubre del 2011) (Esp.).

69. CONSTITUCIÓN ESPAÑOLA, supra nota 63 , art. 148, $\$ 1$, cl.3. 
leyes adicionales para completar los sistemas regionales jurídicos). ${ }^{70}$ Usando este argumento, dos leyes nacionales entraron en vigor en 1990 y 1992, creando un marco jurídico común a pesar de que el gobierno estaba cada vez más descentralizado. ${ }^{71}$ Mientras tanto, el precio del suelo se incrementó fuertemente, especialmente en las grandes ciudades, y el derecho a la vivienda se convirtió en un mito para mucha gente que tuvo que abandonar el centro de las ciudades (en España son las mejores zonas en donde, por lo general, vive la gente con más dinero) y trasladarse a los suburbios (en España son las zonas con menos servicios y por lo tanto la calidad de vida en estas zonas es menor), en busca de viviendas asequibles.

4. La Fragmentación de las Leyes del Uso del Suelo y la Supervivencia de un Núcleo Duro Nacional

En 1997 una decisión sumamente polémica del Tribunal Constitucional Español casi destruye esta estructura legal común. ${ }^{72} \quad \mathrm{El}$ Tribunal declaró que las leyes nacionales de 1990 y 1992 eran parcialmente (aproximadamente el $80 \%$ ) inconstitucionales y, en consecuencia, nulas. ${ }^{73}$ Estableció que la legislación urbanística era una materia de competencia regional y que a nivel nacional solo podía regularse este campo en casos excepcionales (por ejemplo, reglas básicas sobre la expropiación, art. 149.1.18, que están conectadas directamente con los derechos de propiedad). ${ }^{74}$

Como consecuencia, la Ley Nacional de 1998, trató de llenar el vacío que se había creado y aportó algunas reglas generales sobre las clases de suelo y los límites a los planes locales, así como algunas normas sobre expropiaciones. Esta ley fue modificada mediante la Ley de Suelo actual, de 2007, con un contenido similar, pero muy influida por los enfoques de la U.E., como veremos más adelante. ${ }^{75}$

70. Id. art. 149, $\S 1$, cl. 13 (de acuerdo con este artículo, el Parlamento español puede promulgar leyes que establezcan "reglas básicas y la coordinación de la planificación económica general," que son vinculantes para el nivel regional y local).

71. Ver Reforma del Régimen Urbanístico y Valoraciones del Suelo (B.O.E. 1990, 17), disponible en http://www.boe.es./boe/dias/1990/07/27/pdfs/A22060-22073.pdf (última visita 31 de octubre del 2011) (Esp.). Ver también Real Decreto Legislativo por el que se aprueba el Texto Refundido de la Ley sobre el Régimen del Suelo y Ordenacion Urbana (B.O.E. 1992, 156), disponible en $h$ ttp://www.boe.es./boe/dias/1992/06/30/pdfs/A22238-22274.pdf (última visita el 31 de octubre del 2011) (Esp.).

72. S.T.C., el 20 de Marzo del 1997 (B.O.E., No. 61/1997), disponible en http://www.boe.es/boe/dias/1997/04/25/pdfs/T00003-00126.pdf (última visita el 7 de noviembre del 2011) (Esp.).

73. Id.

74. CONSTITUCIÓN ESPAÑOLA , supra nota 63 , art. $149, \S 1$, cl.18.

75. Ley del Suelo (R.D.L. 2007, 8) (España) [en lo sucesivo Ley del Suelo]. 
Más allá de la explicación general anterior, ahora es necesario analizar el actual marco jurídico de la U.E. en el campo específico de prevención de catástrofes, tanto las naturales como las provocadas por el hombre. En este sentido la U.E. ha desarrollado en este ámbito tanto Derecho blando (soft law) como Derecho duro (hard law). ${ }^{76}$

En cuanto a la al primero, el documento más pertinente es el la Comunicación de la Comisión Europea Sobre la Prevención de los Catástrofes Naturales y de los Desastres Provocados por el Hombre del 2009. ${ }^{77}$ En este documento, la Comisión Europea confirma la falta de un enfoque estratégico común para la prevención de estas catástrofes, y desarrolla algunas ideas para su creación. Entre estas ideas, la Comisión Europea recalca tres, en particular: el desarrollo del conocimiento basado en las políticas de prevención de desastres en todos los niveles de gobierno; vincular a los actores y las políticas en todo el ciclo de gestión; y mejorar la eficacia de los instrumentos de política existentes en materia de prevención de desastres. ${ }^{78}$ La Comisión Europea desarrolla diferentes medidas para ser implementadas en el futuro con relación a cada una de estas tres ideas. En relación al desarrollo del conocimiento, la Comisión Europea expresa su intención de desarrollar un inventario completo de las fuentes de información existentes relacionadas con las catástrofes y poner en marcha un grupo de partes interesadas para que revisen la información existente y puedan tomar las medidas necesarias para llenar cualquier vacio de conocimiento que se detecte. ${ }^{79}$ Por otro lado, la Comisión Europea manifiesta su intención de difundir las mejores prácticas, creando

76. Versión Consolidada del Tratado de Funcionamiento de la Unión Europea art. 288, el 5 de septiembre del 2008 (C 83) 171, disponible en http://eur-lex.europa.eu/LexUriServ/ LexUriServ.do?uri=OJ:C:2010:083:0047:0200:en:PDF (23 de septiembre del 2011) [en lo sucesivo TFEU], establece las categorías de decisiones legales de la UE:

Para ejercer las competencias de la Unión, las instituciones adoptarán reglamentos, directivas, decisiones, recomendaciones y dictámenes. El reglamento tendrá un alcance general. Será obligatorio en todos sus elementos y directamente aplicable en cada una directiva obligará, en cuanto al resultado a alcanzar, en cada Estado miembro al que se dirige, pero dejarán a las autoridades nacionales la elección de la forma y los métodos. La decisión deberá ser vinculante en su totalidad. La decisión se designen los destinatarios, sólo será obligatoria para éstos. Recomendaciones y los dictámenes no serán vinculantes. Recomendaciones y los dictámenes son leyes blandas típicas. Aunque la letra del Tratado podría parecer evidente, la realidad es que las recomendaciones y opiniones, así como otras fuentes atípicas de soft law (comunicaciones, por ejemplo) tienen efectos jurídicos de acuerdo con el Tribunal Europeo de Justicia la jurispnudencia. En el caso de Grimaldi, 322/88, de 12 de diciembre de 1989, la Corte subrayó que la ley blanda se deben tener en cuenta por los tribunales de los Estados miembros al aplicar la legislación de la U.E.. Ver SARMIENTO, D., El soft law administrativo, Thomson Civitas, Madrid, 2008, pp. 86 y ff.

77. Prevención de Catástrofes Naturales y de Origen Humano, supra nota 1.

78. Id. en $4, \S 2$.

79. Id. en $4, \S 3.1 .1$. 
directrices sobre la cartografia de peligro/riesgo e incentivando actividades de investigación. ${ }^{80}$

En cuanto a los vínculos entre los actores y las políticas pertinentes, la Comisión Europea ampliará un programa ya establecido de "lecciones aprendidas" de las intervenciones realizadas dentro del marco del Mecanismo Comunitario para la protección civil y también desarrollará cursos específicos sobre la prevención. ${ }^{81}$ Por otra parte, la Comisión Europea aumentará la conciencia del público en general con relación a los desastres. $^{82}$ Además, creará una red que cubrirá los departamentos encargados de la planificación del suelo, del riesgo y mapas de peligros naturales, de la protección del medio ambiente, y de la preparación y respuesta para emergencias al mismo tiempo que refuerza el vínculo entre los sistemas de alerta temprana. ${ }^{83}$

En relación con la mejora de la eficacia de los instrumentos de política existentes, la Comisión Europea, en estrecha cooperación con los Estados miembros, desarrollará diversas políticas públicas para fortalecer la integración de la prevención de desastres en la programación operativa nacional de la financiación de la U.E. ${ }^{84}$ Por último, la legislación comunitaria vigente tendrá en cuenta la prevención de catástrofes durante la revisión prevista de una serie de elementos de la legislación de la U.E.. La Comisión Europea animará a los Estados Miembros a integrar plenamente los códigos europeos comunes de diseño de edificios y obras civiles en sus normas de planificación nacional para mitigar los impactos de los terremotos. ${ }^{85}$

En cuanto a normas vinculantes (el Derecho duro o hard law), la U.E. ya ha desarrollado un conjunto de instrumentos para abordar diferentes aspectos de la prevención de catástrofes naturales, nuestro principal interés, y de la preparación respuesta y recuperación de las mismas. Estos instrumentos jurídicos son, básicamente, directivas ${ }^{86}$ y también

\footnotetext{
80. Id. en $5, \S 3.1 .1$.

81. Id. en 6, § 3.2.1.

82. Prevención de Catástrofes Naturales y de Origen Humano, supra nota 1, en 6, § 3.2.2.

83. Id. en $6, \S 3.2 .3$.

84. Id. en $7, \S 3.3 .1$.

85. Id. en 8, § 3.3.2.

86. Ver, por ejemplo, Directiva del Consejo 2009/71, 2009 O.J. (L 172) 18 (EURATOM), disponible en http://eur-lex.europa.eu/LexUriServ/LexUriServ.do?uri=OJ:L:2009:172:0018:0022: ES:PDF (última visita 31el de octubre del 2011); Directiva del Consejo 2007/60, 2007 O.J. (L. 288) 27 (EC), disponible en http://eur-lex.europa.eu/LexUriServ/LexUriServ.do?uri=OJ:L:2007:288:0027:0034: ES:PDF (última visita el 31 de octubre del 2011); Directiva del Consejo 96/82, 1996 O.J. (L 010) 13 (EC), disponible en hitp://eur-lex.europa.eu/LexUriServ/LexUriServ.do?uri=CELEX:31996L0082: ES:HTML (última visita el 31 de octubre del 2011).
} 
regulaciones, ${ }^{87}$ como veremos más adelante cuando tratemos con cuestiones más específicas. Desde el punto de vista normativo, probablemente las herramientas más relevantes son las directivas de la U.E., las cuales "serán obligatorias, en cuanto al resultado a alcanzar, en cada Estado miembro" al que están dirigidas, pero "dejarán a las autoridades nacionales la elección de qué forma y qué medios utilizar." ${ }^{88}$

\section{EVALUACIÓN Y GESTIÓN DE LOS RIESGOS POR LA LEY DEL USO DEL SUELO: EL PROBLEMA ESPECÍFICO DE INCERTIDUMBRE CIENTÍFICA}

\section{A. Incertidumbre Cientifica y Principio de Precaución}

De acuerdo con la legislación de la U.E. y los marcos jurídicos de los Estados Miembros (como España), la planificación urbana debe evaluar los riesgos para prevenir las catástrofes naturales o las provocadas por el hombre. Pero a veces, en la identificación de un fenómeno, un producto o un proceso, la evaluación científica no permite que se determine el riesgo con suficiente certeza. ${ }^{89} \mathrm{La}$ incertidumbre científica puede surgir de la controversia sobre los datos existentes o la falta de algunos datos pertinentes. ${ }^{90}$ En estas situaciones, el marco jurídico de la U.E. exige el uso del principio de precaución. ${ }^{91}$ La Comunicación de la Comisión sobre el Principio de Precaución (02.02.200 Bruselas COM (2000) I) es el documento de Derecho blando (soft law) de la U.E. más importante en ese

87. Ver, por ejemplo, Reglamento de la Comisión 1891/2006, 2006 O.J. (L 394) 1 (EC), disponible en http://eurlex.europa.eu/LexUriServ/LexUriServ.do?uri=OJ:L:2006:394:0001:0004:EN:PDF (ultima visita el 27 de octubre del 2011) (relativo a la financiación plurianual de la actuación de la Agencia Europea de Seguridad Marítima en el ámbito de la lucha contra la contaminación por los buques); Reglamento de la Comisión 1726/2003, 2003 O.J. (L 249) 1 (EC), disponible en http://eurlex.europa.eu/LexUriServ/LexUriServ.do?uri=OJ:L:2003:249:0001:0004:EN:PDF (última visita el 27 de octubre del 2011) (prohibiendo los petroleros monocasco a partir de los puertos europeos).

88. TFEU, supra nota 76, art. 288.

89. Comunicación de la Comisión Sobre el Recurso al Principio de Precaución, en 4, COM (2000) 1 (el 2 de febrero del 2000), disponible en http://ec.europa.eu/dgs/ health_consumer/library/pub/pub07_en.pdf (última visita el 27 de octubre del 2011) (este documento sólo trena de ayudar en la interpretación de un principio jurídicamente vinculante en el art. 191 del Trenado y ya aplicado por el Tribunal Europeo de Justicia jurisprudencia (por ejemplo los casos C157/96 y C-180/96), donde la Corte reviso la validez de la decisión de la Comisión de prohibir la exportación de carne del Reino Unido para reducir el riesgo de transmisión de la EEB, la situación conocida como "vacas locas") [en lo sucesivo Principio de Precaución].

90. Id. en 14.

91. TFEU, supra nota 76 , art. 191. 
campo. ${ }^{92}$ Ofreceré una versión general de este documento en las siguientes líneas.

De acuerdo con la citada Comunicación, el principio de precaución debe ser considerado dentro de un enfoque estructurado para el análisis de riesgos, el cual contiene tres elementos: evaluación, gestión y comunicación de riesgos. ${ }^{93}$ La gente que toma las decisiones tiene que ser consciente del grado de incertidumbre en cuanto a los resultados de la evaluación de la información científica disponible. ${ }^{94}$ Juzgar lo que sea un nivel "aceptable" de riesgo para la sociedad es una responsabilidad política. ${ }^{95}$ En algunos casos, la respuesta correcta puede ser no actuar o al menos no introducir una medida legal vinculante. ${ }^{96}$ En el caso de actuar, una amplia gama de iniciativas están disponibles: desde una medida legal vinculante hasta un proyecto de investigación o de recomendación. ${ }^{97}$

Con el fin de elegir entre las posibles acciones, las medidas basadas en el principio de precaución se guían por una serie de principios: ${ }^{98}$

a) Proporcionalidad: la acción elegida debe alcanzar el nivel adecuado de protección. ${ }^{99}$ El principio de proporcionalidad es un principio legal fundamental en el Derecho Europeo. ${ }^{100}$ La revisión judicial basada en este principio utiliza tres "filtros" o fases:

i) Los medios adoptados deben ser adecuados para la protección: No pueden ser extremos, en el sentido de restringir los derechos innecesariamente, ni insuficientes, en el sentido de que no protejan el interés público. ${ }^{101}$ En algunos casos, la prohibición total no puede ser una respuesta proporcional a un riesgo potencial. ${ }^{102}$ En otros casos, puede ser la única respuesta efectiva posible para un riesgo potencial. ${ }^{103}$

92. Ver Principio de Precaución, supra nota 89, en 4.

93. Id. en 3.

94. Ver id. en 17.

95. Id. en 16.

96. Ver id. en 15.

97. Ver Principio de Precaución, supra nota 89, en 16.

98. Id. en 4.

99. Id. en 18.

100. Ver, por ejemplo, TFEU, supra nota 76, art. 296.

101. Ver Principio de Precaución, supra nota 89, en 18. Ver también ConstITUCIÓN ESPAÑOLA, supra nota 63 , art $16, \S 1$.

102. Principio de Precaución, supra nota 89, en 18.

103. Id. 
ii) La restricción de los derechos debe ser necesaria. ${ }^{104}$ Esto significa que las autoridades públicas deben estar satisfechas con los medios más leves si es que son efectivos. Todas las medidas excesivas que restringen la libertad deben ser evitadas y si se utilizan pueden ser consideradas ilegales por medio de revisión judicial. $^{105}$

iii) El tercer filtro es la proporcionalidad en sentido estricto. Esto significa que los beneficios (de todo tipo) asociados a la intervención pública deben ser mayores que los costes inherentes (de todo tipo).

b) La no discriminación: otro medio clásico para controlar la acción pública en Europa. ${ }^{106}$ Situaciones comparables no deben ser tratadas de modo diferente y situaciones diferentes no deben ser tratadas de la misma manera a menos de que existan fundamentos objetivos para hacerlo (los cuales deben ser explicados de acuerdo al deber legal de explicar las razones que los justifican). ${ }^{107}$ Por otro lado, la no discriminación no prohíbe acciones positivas (el termino Europeo para acciones afirmativas o discriminación invertida) de acuerdo a las circunstancias. ${ }^{108}$

c) Consistencia: las medidas deben ser consistentes con las medidas ya adoptadas en circunstancias similares o usando aproximaciones similares. ${ }^{109}$

d) Análisis de coste-beneficio: la evaluación de los pros y los contras no se pueden reducir a un análisis de costebeneficio económico. ${ }^{110}$ La evaluación debe ser más amplia en su alcance e incluir consideraciones no económicas. ${ }^{111}$ Aunque deberá incluir el análisis costebeneficio cuando sea posible y apropiado. ${ }^{12}$

104. Ver generalmente Carta de Derechos Fundamentales de la Unión Europea art. 52, el 18 de diciembre del 2000, O.J. (C 364) 1, disponible en http://www.europarl.europa.eu/charter/pdf/text_es.pdf (última visita el 27 de octubre del 2011) [en lo sucesivo Derechos Fundamentales].

105. Ver generalmente id. Ver también Principio de Precaución, supra nota 89, en 16.

106. Ver Derechos Fundamentales, supra nota 104, art. 21. Ver también CONSTITUCIón ESPAÑOLA, supra nota 63, arts. 9.2-14.

107. Principio de Precaución, supra nota 89, en 19.

108. Ver, por ejemplo, Prof. O. DE SCHUTTER, THE PROHIBITION OF DISCRIMINATION UNDER European human Rights law [La Prohibición de la Discriminación en la ley Europea de DERECHOS HUMANOS] (Eur. Comm'n Emp't \& Soc. Aff., febrero del 2005), disponible en ec.europa.eu/social/BlobServlet?docId=1692\&langId=en (última visita el 27 de octubre del 2011).

109. Principio de Precaución, supra nota 89, en 19.

110. Id.

111. Id.

112. Id. 
e) Examen de los avances científicos: las medidas deben mantenerse mientras los datos científicos sean inadecuados, imprecisos o no concluyentes, y mientras el riesgo se considere demasiado alto para ser impuesto a la sociedad. $^{113}$ Las medidas basadas en el principio de precaución deben ser revisadas y modificadas si es necesario en función de los resultados de la investigación científica y del seguimiento de su impacto. ${ }^{114}$

f) Carga de la prueba: las medidas basadas en el principio de precaución pueden asignar la responsabilidad de aportar pruebas científicas necesarias para una evaluación completa del riesgo. ${ }^{115}$ Es posible solicitar la aprobación administrativa previa para la actividad y trasladar la carga de la prueba hacia el individuo, quien debe dejar claro que no hay ningún riesgo inaceptable de causar desastres. ${ }^{116}$

\section{B. Evaluación}

Considerando, si es necesario, el principio de precaución, los estados miembros de la U.E. han establecido regulaciones con relación al deber público de evaluar riesgos en la planificación del uso del suelo. ${ }^{117}$ En esta sección voy a usar el ejemplo de España. Como marco general, el artículo 10 de la Ley de Suelo española establece que la prevención de riesgos naturales y accidentes graves es uno de los principios legales vinculantes que deben guiar la regulación pública del suelo en todos los casos. ${ }^{18}$

\section{Riesgos Naturales: Las Directivas de la U.E. y Su Impacto a Nivel Nacional: el Caso Español}

Como se ha explicado anteriormente, la U.E. ha aprobado varias directivas que son obligatorias en cuanto al resultado a ser alcanzado por cada Estado Miembro al que se dirigen, pero que permiten la elección de la forma y métodos a las autoridades nacionales. ${ }^{119}$ Esta técnica jurídica clásica de la U.E. explica porque España, al igual que los demás Estados miembros, se ha visto obligada a trasponer varias directivas, aprobando la nueva legislación en relación a la prevención y gestión de catástrofes. ${ }^{120}$

113. Id. en 20.

114. Principio de Precaución, supra nota 89, en 21.

115. Id. en 22.

116. Ver id. en 21.

117. Ver, por ejemplo, Principio de Precaución, supra nota 89, anexo $\mathrm{I}$.

118. Ley del Suelo, supra nota 75, art. 10(c).

119. Ver discusión infra Parte II.B.2.f.

120. Id. 
2. Riesgos Naturales: los requisitos legales de evaluación según la planificación: Evaluación del Impacto Ambiental y legislación del suelo Española

\section{a. Evaluación del Impacto Ambiental y legislación nacional del suelo Española}

Las directivas sobre la Evaluación del Impacto Ambiental son buenos ejemplos de la influencia de la U.E. en los Estados Miembros. ${ }^{121}$ Entre estos, quiero resaltar la Directiva de Evaluación Ambiental Estratégica. ${ }^{122}$ Esta Directiva ha sido transpuesta en España por medio de la Ley 9 / 2006 sobre evaluación ambiental de planes y programas en el medio ambiente. ${ }^{123}$ Esta ley exige un procedimiento administrativo para la evaluación del impacto ambiental causado por la planificación urbana. ${ }^{124}$ Con relación a lo dicho, la ley de suelo del 2007 establece la obligación de incluir mapas de riesgo en el informe de sostenibilidad ambiental que el promotor debe preparar. ${ }^{125}$ Este informe debe ser incluido en un documento denominado Memoria Ambiental, el cual debe explicar el impacto ambiental. ${ }^{126}$

\section{b. Prevención de riesgos de inundación y planificación urbana}

Con respecto a la prevención de los riesgos de inundación y la planificación urbana, el reglamento español Real Decreto 903/2010 traspone la directiva de la U.E. 2007/60/CE sobre la evaluación y gestión de los riesgos de inundación. ${ }^{127}$ De acuerdo con esta Directiva, la norma

121. Ver generalmente European Commission on Environment: Environmental Impact Assessment [Comisión Europea sobre el Medioambiente: Evaluación de Impacto Ambiental], http://ec.europa.eu/environment/eia/home.htm (última visita 27 de octubre del 2011).

122. Ver Council Directive 2001/42/EC of the European Parliament and of the Council of 27 June 2001 on the Assessment of the Effects of Certain Plans and Programmes on the Environment [Directiva 2001/42 del Parlamento Europeo y del Consejo de 27 del Junio 2001 sobre la Evaluación de los Efectos de Ciertos Planes y Programas en el Medio Ambiente], 2001 O.J. (L 197) 30 (EC), disponible en http://eur-lex.europa.eu/LexUriServ/LexUriServ.do?uri=OJ:L:2001:197:0030:0037: EN:PDF (última visita el 12 de noviembre del 2011).

123. Evaluación de los Efectos de Determinados Planes y Programas en el Medio Ambiente (B.O.E. 2006, 9), disponible en http://www.seo.org/media/docs/ley_planes_programas_ma.pdf (última visita el 31 de octubre del 2011) (Esp.) [en lo sucesivo Ley 9/2006].

124. Ley del Suelo, supra nota 75, art. 15, § 1. Ver también Ley 9/2006, supra nota 123 , art. 7 , $\S 1$.

125. Ver Ley del Suelo, supra nota 75 , art. $15, \S 2$.

126. Id. art. $15, \S 3(\mathrm{c})$.

127. Ver Evaluación y Gestión de Riesgos de Inundación (B.O.E. 2010, 903), disponible en http:/www.boe.es/boe/dias/2010/07/15/pdfs/BOE-A-2010-11184.pdf (última visita el 24 de octubre del 2011) (Esp.). Ver también Comunicación de la Comisión al Consejo, El Parlamento Europeo, El Comité Económico, y Social Europeo y El Comité de las Regiones, COM (2004) 472 final (7 de 
española crea diferentes instrumentos para evaluar los riesgos de inundación; mapas de peligro de inundaciones, mapas de riesgo de inundaciones y mapas de gestión. ${ }^{128}$ La norma establece claramente que la planificación urbanística está sujeta a estos tres tipos de mapas. Los planes regionales y locales, al regular el uso del suelo, no pueden incluir decisiones contra la gestión de inundaciones, y la construcción estará prohibida en terrenos con riesgos de inundaciones identificados. ${ }^{129}$

3. Riesgos Creados Por el Hombre: el Ejemplo de la Evaluación de los Riesgos de Crimen y Terrorismo

La necesidad de una evaluación durante el proceso de planificación urbana se extiende a los riesgos creados por el hombre. Un buen ejemplo es la evaluación obligatoria de los riesgos de delincuencia y de terrorismo en la planificación urbana establecidos en la legislación de Cataluña y otras legislaciones europeas (por ejemplo, Francia). ${ }^{130}$ Voy a considerar este tipo de prevención de riesgos en la última sección del estudio.

\section{Gestión}

En algunos casos, la legislación evalúa los riesgos y decide cómo tratarlos. Las medidas que pueden llegar a tomarse varían desde el establecimiento de directrices a seguir por futuros planes locales, a prohibiciones legales para los propietarios del suelo con el fin de evitar factores que pueden llevar a una catástrofe natural.

\section{Prohibiciones Legales Vinculantes para la Planificación Urbanística}

Un buen ejemplo de esta técnica es el artículo 12 de la Ley del Suelo española, la cual estipula que el desarrollo urbano está prohibido en terrenos con riesgos naturales o tecnológicos. ${ }^{131}$ Este tipo de terreno se considera suelo rural. ${ }^{132}$ Por lo tanto, existe una prohibición legal de construir en él y ello debe constar en la planificación urbana (hay que tener en cuenta que no existe una compensación legal por esta prohibición). ${ }^{133}$

diciembre del 2004), disponible en http://eur-lex.europa.eu/LexUriServ/ LexUriServ.do?uri= COM:2004:0472:FIN:es:PDF (última visita el 28 de octubre del 2011).

128. Directiva del Consejo 2007/60, 2007 O.J. (L 288) 27 (EC), disponible en http://eurlex.europa.eu/LexUriServ/LexUriServ.do?uri=OJ:L:2007:288:0027:0034:EN:PDF (última visita el 27 de octubre del 2011).

129. Ver id. $\$ 13$.

130. Ver discusión en Parte $V$.

131. Ley del Suelo, supra nota 75 , art. $12, \S 2(\mathrm{a})$.

132. Ver id. art. $12, \S 2$.

133. Ver id. art. $3, \S 1$. 
2. Prohibiciones Legales Vinculantes Para La Planificación Urbanística $Y$ Propietarios Privados: La Función Social De La Propiedad Y La Prevención De Catástrofes Naturales

\section{a. Planificación Regional y Zonificación Local}

Los planes urbanísticos locales pueden estar vinculados por directrices provenientes de la planificación regional. La ordenación del territorio en España no está en manos del Estado. ${ }^{134}$ La Constitución Española y los diferentes Estatutos de Autonomía establecen que la ordenación territorial es un poder regional y por tanto las Comunidades Autónomas promulgan leyes específicas regulando este ámbito. ${ }^{135}$ Pero el Tribunal Constitucional ha confirmado que el Estado mantiene la capacidad de influir "de facto" en la ordenación regional mediante la decisión sobre la ubicación de infraestructuras estatales (por ejemplo, el tren de alta velocidad, AVE, que actualmente es un elemento básico en la cohesión territorial por medio de decisiones sobre sus vías y estaciones en todo el territorio Español). ${ }^{136}$

En el ámbito de las Comunidades Autónomas, es importante considerar el ejemplo catalán. El parlamento catalán promulgó una ley en 1983 sobre ordenación territorial de acuerdo con su competencia en este ámbito, ${ }^{137} \mathrm{y}$ ha continuado desarrollando una nueva generación de planes de ordenación territorial, vinculando a las autoridades locales en relación al uso del suelo y las viviendas desde el 2004 (incluyendo planes de protección del territorio costero). ${ }^{138}$ El último paso en este reciente proceso ha sido la Ley sobre el Derecho a la Vivienda del 2007, la cual ha creado el Plan territorial sectorial de vivienda, pendiente de una futura aprobación. 139 Este tipo de plan regional puede contener provisiones de prevención de

134. Ver, por ejemplo, PONCE SOLE ET AL., supra nota 45.

135. Ver CONSTITUCIÓN ESPAÑOLA, supra nota 63, art. 148, § 1, cl. 3.

136. Ver S.T.C., 13 de mayo del 1986 (B.O.E., No. 56/1986), disponible en http://boe.es/boe/dias/1986/06/13/pdfs/T00001-00005.pdf (última visita el 27 de octubre del 2011) (Esp.).

137. Ver Política Territorial (Diari Oficial de la Generalitat de Catalunya 1983, 230) [Diario Oficial de la Generalidad de Cataluña] (Cataluña), disponible en http://www.parlament.cat/ web/activitat-parlamentaria/leis?p_pagina=1\&p_fw= (última visita el 29 de octubre del 2011) (seguir "Llei 23/1983, del 21 del noviembre, de política territorial" hiperenlace) (Esp.).

138. Ver generalmente Oriol Nel·lo, La Nueva Politica Territorial de Cataluña, GENERALITAT DE CATALUNYA (septiembre del 2005), http://www.apgeo.pt/files/docs/CD_X_Coloquio_Iberico_ Geografia/pdfs/106.pdf (última visita el 30 de octubre del 2011).

139. Ver Llei del Dret a l'Habitatge de Catalá [Ley del Derecho a la Vivienda de Cataluña] art. 11 (B.O.E. 2007, 18), disponible en http://www20.gencen.cen/docs/habitenge/Home/ Secretaria\%20dhabitenge/Publicacions/Llei\%2018_2007\%20del\%20dret\%20a\%20lhabitenge/docs/43_1 54955.pdf (última visita el 30 de octubre del 2011) (Esp.). 
catástrofes naturales. Un ejemplo son las Directrices Generales en Canarias. ${ }^{140}$ Éstas pueden establecer criterios para prevenir "riesgos de catástrofes naturales" obligatorios para todos los planes locales urbanísticos. $^{141}$

\section{b. Limites al Derecho de Propiedad y las Catástrofes Naturales}

En España, los propietarios de suelos no pueden hacer lo que quieran con su propiedad. Aunque la Constitución Española (CE) reconoce y protege el derecho de propiedad, éste debe ser desarrollado de acuerdo con su "función social."142 Esto significa que la legislación (y los planes urbanísticos desarrollados a su amparo) puede limitar el derecho de propiedad estableciendo determinadas obligaciones. ${ }^{143}$ Estas obligaciones no suponen una expropiación de la propiedad y por tanto no existe una compensación como contraprestación. ${ }^{144}$ Un ejemplo de ello es el artículo 9 de la Ley del Suelo española, la cual establece la obligación de conservación de sus propiedades a todos los propietarios de terrenos y edificios. ${ }^{145}$ Esta obligación legal lleva incluida la obligación de mantener el suelo rural en buenas condiciones con el fin de evitar cualquier posible riesgo de catástrofe natural (fuegos, inundaciones, etc.). ${ }^{146}$

\section{Planificar Y Reclamar Daños Y PerJuicios En Caso De DESASTRES NATURALES}

\section{A. Falta de Prevención: Responsabilidad Pública Y Privada}

Las posibles acciones legales por daños y perjuicios están establecidas de acuerdo con cada sistema legal nacional. Claramente el sistema legal Americano difiere de los Europeos. ${ }^{147}$ La responsabilidad privada y la pública pueden tener distintos regímenes, de acuerdo con los sistemas de Derecho administrativo de cada país en Europa. Incluso con esta distinción

\footnotetext{
140. Ver Ley por la que se aprueban las Directrices de Ordenación General y del Turismo de Canarias (Boletin Oficial de Canarias, 2003, 19), disponible en http:/www.gobiernodecanarias.org/boc/2003/073/boc-2003-073-002.pdf (última visita el 24 de octubre del 2011) (Esp.).

141. Ver id. directriz 3(e).

142. Ver CONSTITUCIÓN ESPAÑOLA, supra nota 63, art. 33.

143. Id.

144. Id.

145. Ley del Suelo, supra nota 75 , art 9.

146. Id.

147. Ver, por ejemplo, RICHARD J. PIERCE, JR., ADMINISTRENIVE LAW [Ley Administrativa] 167 (Fountain Press 2008) (proporcionando un resumen de las acciones de responsabilidad contra agencias y funcionarios estadounidenses).
} 
público-privado en mente, las características legales pueden ser bastante distintas (como, por ejemplo en el caso de Inglaterra y España, país éste donde, como veremos, existe una responsabilidad pública "objetiva" que no requiere falta administrativa o negligencia). ${ }^{148}$

En el caso Europeo tenemos una pluralidad de regulaciones de responsabilidad, con orígenes en los distintos sistemas legales de cada país. Pero existe un mínimo marco legal común Europeo creado por la Directiva 2004/35/EC del Parlamento Europeo y del Consejo de Responsabilidad Medioambiental en Relación con la Prevención Y Reparación de Daños Medioambientales. ${ }^{149}$ Este marco común se aplica a la responsabilidad privada. ${ }^{150}$ Los estados miembros se vieron obligados a trasponer la Directiva antes del 30 de abril de 2007..$^{151}$ Espana cumplió su obligación mediante la Ley 26/2007, de 23 de Octubre del mismo año. ${ }^{152}$

Esta Directiva establece un marco común Europeo para la responsabilidad medioambiental en caso de daños medioambientales, basándose en el principio de "el que contamina paga." cubre actividades profesionales enumeradas en el Anexo III. ${ }^{154}$ Éste incluye principalmente actividades agrícolas $o$ industriales que requieren una licencia para llevarse a cabo. ${ }^{155}$ Estas actividades se consideran responsabilidad del operador incluso si no ha sido el culpable directo del daño. ${ }^{156}$ Fuera del Anexo III hay otras actividades que pueden dañar especies u otros hábitats naturales protegidos por la legislación Europea. ${ }^{157}$ En el segundo de los casos, el operador será considerado responsable si ha sido culpable o ha incurrido en negligencia. ${ }^{158}$ En ambos casos, la Directiva no cubrirá daños medioambientales causados por "un fenómeno natural de carácter excepcional, inevitable e irresistible." 159 No cubre daños causados por riesgos nucleares. ${ }^{160} \mathrm{La}$ autoridad competente establecerá qué

148. Id.

149. Directiva del Consejo 2004/35, 2004 O.J. (L 143) 56 (EC), disponible en http://eurlex.europa.eu/LexUriServ/LexUriServ.do?uri=OJ:L:2004:143:0056:0075:EN:PDF (última visita el 27 de octubre del 2011) [en lo sucesivo Directiva 2004/35].

150. Ver Responsabilidad Medioambiental pmbl., $§ 6$ (B.O.E. 2007, 26), disponible en http://boe.es/boe/dias/2007/10/24/pdfs/A43229-43250.pdf (última visita el 27 de octubre del 2011) (Esp.) [en lo sucesivo Responsabilidad Medioambiental].

151. Directiva 2004/35, supra nota 149, art. 19, $\S 1$.

152. Ver Responsabilidad Medioambiental, supra nota 150 , pmbl., $\S 1$.

153. Directiva 2004/35, supra nota 149 , art. 1.

154. Ver id. art. $3, \$ 1$ (a).

155. Ver id. anexo II.

156. Ver id. art. $3, \S 1(\mathrm{a})$.

157. Ver id. art. $2, \S 1(\mathrm{a})$.

158. Ver Directiva 2004/35, supra nota 149 , art. $3, \S 1$ (b).

159. Id. art. $4, \S 1$ (b).

160. Id. art. $4, \S 4$. 
profesional ha causado el daño, evaluará la importancia del daño y determinará qué medidas correctivas se deberán de tomar. ${ }^{161}$ La parte interesada tendrá acceso a un tribunal u otro órgano público independiente e imparcial con competencia para decidir sobre la legalidad de la decisión pública. ${ }^{162}$

En relación con la responsabilidad pública por falta de una adecuada evaluación de los riesgos, no existe un marco legal común Europeo. Como consecuencia, cada Estado Miembro aplicará su propio sistema legal. En el caso de España, el artículo 106.2 de la Constitución y los artículos 139 y siguientes de la ley 30/1992 establecen el marco legal aplicable a todas las administraciones públicas de España, y respecto a todas actividades de todos los sectores. ${ }^{163}$ De acuerdo con este régimen legal, una administración pública será legalmente responsable si hay un daño real que los ciudadanos no tienen la obligación jurídica de soportar, si la actividad administrativa - o la falta de actividad administrativa, cuando la acción era obligatoria por ley - está implicada en el daño y si existe una relación de causalidad entre la actividad pública, o la falta de actividad pública, y el daño. ${ }^{164}$ En España, la culpa 0 negligencia no son necesarias para reconocer la responsabilidad administrativa ${ }^{165} \mathrm{El}$ pago se realiza a través de los presupuestos públicos: como regla general, los funcionarios administrativos no tienen que pagar dinero de sus bolsillos, con la excepción del caso en que una administración pública paga por los daños causados por la negligencia o culpa grave de su empleado público. En estos supuestos, la administración pública debe recuperar la suma pagada del bolsillo del empleado público. ${ }^{166}$

Una cuestión que puede surgir en caso de catástrofes y daños y perjuicios es si la actividad pública de prevención es adecuada. En otras palabras, ¿debe la administración pública ser responsable de los daños y perjuicios si la planificación urbana no evaluó debidamente los riesgos naturales o los provocados por el hombre y hay daños como consecuencia de un desastre?

161. Ver id. art. 11, §§1-2.

162. Id. art. $13, \S 1$.

163. Ver CONSTITUCIÓN ESPAÑOLA, supra nota 63, § 106(2). Ver también Régimen Jurídico de las Administraciones Públicas y del Procedimiento Administrativo Común art. 139 (B.O.E. 1992, 30) (Esp.) [en lo sucesivo Régimen Jurídico 1992].

164. Ver Régimen Jurídico1992, supra nota 163, art. 130.

165. Id.

166. Id. art. 145.2. 
B. Algunos ejemplos españoles acerca de las reclamaciones de indemnización en caso de catástrofes

\section{El camping de Biescas}

Este desastre en 1996 tuvo un gran impacto en la sociedad española. ${ }^{167}$ Una inundación repentina en el barranco de Arás destruyó el camping de Biescas resultando en 87 muertes. ${ }^{168}$ Después de largas discusiones sobre la definición de este desastre como un caso de fuerza mayor (act of God) se presentó una demanda contra las autoridades públicas. ${ }^{169}$ Una decisión de un tribunal español en 2005 trató directamente la cuestión de la posible existencia de responsabilidad pública debido a la falta de evaluación adecuada de los riesgos al permitir la construcción del camping en el barranco. ${ }^{170} \mathrm{Y}$ el resultado fue positivo: la administración estatal y regional fueron declaradas culpables y la indemnización por daños y perjuicios fue reconocida. ${ }^{171}$

Esta decisión judicial digna de mención señaló que la administración pública no evaluó los existentes "riesgos naturales y artificiales de todo tipo" para las personas y sus pertenencias. ${ }^{172}$ Según la Audiencia Nacional española, las administraciones públicas están siempre obligadas a prevenir los riesgos; incluso donde hay una falta de disposición legal específica, existe ese deber. Debido al hecho de que el desastre no fue ni imprevisible ni inevitable, los poderes públicos fueron obligados a indemnizar por daños y perjuicios por un importe total de 12 millones de euros. ${ }^{173}$

Este desastre provocó una serie de medidas para desarrollar una política de prevención de desastres, por ejemplo, la modificación de la Ley española de Suelo y la creación de una Comisión Parlamentaria Especial sobre la Prevención y asistencia en casos de catástrofes. ${ }^{174}$

167. A.N., 21 de diciembre de 2005 (R.O.J., No. 1976), supra nota 3.

168. Id.

169. Id.

170. Id.

171. Id.

172. A.N., 21 de diciembre de 2005 (R.O.J., No. 1976), supra nota 3.

173. Id.

174. Ver Informe de la Comisión Especial sobre la Prevención y Asistencia en Situaciones de Catástrofe, 9 de diciembre de 1998 (Boletín Oficial de las Cortes Generales, No. 596), disponible en http://www.iaem.es/Documentos/InformeSenadoPrevCatastrofes1998.pdf (última visita el 29 de septiembre del 2011) (Esp.). 
2. El Atentado de un Coche Bomba contra un Cuartel de la Guardia Civil

Una segunda decisión judicial interesante relativa a la responsabilidad pública en la prevención de desastres es la decisión del Tribunal Supremo español de 2 de noviembre de 2004. ${ }^{175}$ En este caso, los magistrados consideraron el ataque de un coche bomba contra un cuartel de las fuerzas de seguridad españolas (la Guardia Civil). La explosión causó una muerte. ${ }^{176}$

El demandante argumentó que los daños personales y materiales fueron causados por la falta de un plan adecuado para introducir las medidas de seguridad. Un ataque anterior se había intentado en contra de la misma comisaría, un año antes. ${ }^{177}$ El Tribunal Supremo español rechazó la reclamación por daños y perjuicios, argumentando que a pesar de que era posible planificar más medidas de seguridad en el diseño urbano, los daños fueron causados por los terroristas y no debido a la falta de acción pública. En el régimen jurídico español este caso es calificado como la rotura del nexo causal: puede haber existido una mala administración, pero la causa de los daños y perjuicios no es esta mala administración, sino la actividad de la víctima $o$ un tercero. ${ }^{178}$

\section{LA ORDENACIÓN DEL TERRITORIO Y LA PREVENCIÓN DE DESASTRES}

A. La ubicación de las Centrales Nucleares y la Prevención de Riesgos a Través de la Planificación Urbana: Los Procedimientos Administrativos y los Derechos Fundamentales.

La prevención de desastres causados por la energía nuclear es una preocupación importante, como lo demuestra las tragedias de Chernobyl (1986) y Fukushima (2011). ${ }^{179}$ La Agencia Internacional de Energía Atómica ha desarrollado los requisitos de seguridad en relación con la ubicación de las centrales nucleares. En el ámbito europeo, las centrales nucleares producen actualmente alrededor de un tercio de la electricidad y el $15 \%$ de la energía consumida en la Unión Europea, de acuerdo con

175. Jurisprudencia del Tribunal Supremo, 2 de noviembre de 2004 (España).

176. Id.

177. Id.

178. Id.

179. Ver generalmente BELARUS FOREIGN MINISTRY [MINISTERIO DE RELACIONES EXTERIORES DE Belarús], CHERnOBYL Disaster [DESASTRE DEL CHERNBOYL] (Abril 2009), disponible en http://chernobyl.undp.org/english/docs/belarus_23_anniversary.pdf (última visita el 28 del octubre de 2011). 
información de la Comisión Europea. ${ }^{180}$ Esto explica la Directiva Euratom, la cual establece un marco comunitario para la seguridad de las instalaciones nucleares. Ésta es de trasposición obligatoria para los Estados miembros antes del 22 de julio de $2011 .^{181}$ Sin embargo, la presente Directiva no dice nada acerca de la ubicación de las centrales nucleares. Nos recuerda que "la responsabilidad nacional de los Estados miembros de la seguridad nuclear de las instalaciones nucleares es el principio fundamental sobre el cual la reglamentación de la seguridad nuclear se ha desarrollado a nivel internacional," y ello sigue siendo cierto a nivel europeo. ${ }^{182}$

Por lo tanto, es necesario ir a los ordenamientos jurídicos nacionales de los países de la U.E. para saber sobre la evaluación de estos riesgos y la planificación urbana. En el caso español, de acuerdo con el reglamento 1836/1999, antes de obtener una decisión administrativa para la autorización de una planta nuclear, es necesario obtener una "licencia previa o licencia de ubicación," que es un reconocimiento por parte de las autoridades públicas de la posibilidad de la ubicación de una planta nuclear en un lugar específico. ${ }^{183}$ Después de obtener esta licencia, y sólo entonces, el titular de la licencia anterior puede efectuar la solicitud para obtener un permiso de construcción para la edificación de la instalación nuclear. ${ }^{184}$

El interesado deberá solicitar la licencia anterior, mediante la presentación de varios documentos, incluyendo una evaluación de los posibles riesgos detectados. ${ }^{185}$ Después de un procedimiento administrativo, con consulta pública, el Ministerio de Industria concederá o denegará la solicitud de la licencia. ${ }^{186}$ Por lo tanto, estamos ante el ejercicio de una potestad discrecional. En este contexto, el papel y la importancia del debido procedimiento administrativo para garantizar los derechos de los ciudadanos y la buena administración - es decir, el ejercicio de las facultades discrecionales que respeten la obligación de la diligencia debida - es esencial. La importancia de los procedimientos administrativos en la

180. Comunicación de la Comisión al Parlamento Europeo y al Consejo, Una Politica Energética para Europa, en 17, art. 3.8, COM (2000) 1 final (Oct. 1, 2007), disponible en http://eurlex.europa.eu/LexUriServ/site/en/com/2007/com2007_0001en01.pdf (última visita el 29 de septiembre del 2011).

181. Directiva del Consejo 2009/71, art. 8, 2009 O.J. (L 172) 18 (EURATOM), disponible en http://eur-lex.europa.eu/LexUriServ/LexUriServ.do?uri=OJ:L:2009:172:0018:0022:EN:PDF (última visita el 20 de octubre del 2011) [en lo sucesivo EURATOM 2009/71].

182. Id.

183. Reglamento Sobre Instalaciones Nucleares y Radiactivas (B.O.E. 1999, 1836) (Esp.) [en lo sucesivoDecreto Real 1836/1999].

184. Id.

185. Id.

186. Id. 
protección de los derechos fundamentales fue subrayada por una famosa decisión del Tribunal Constitucional alemán sobre la planta nuclear de Mülheim-Kärlich. ${ }^{187}$

En esa decisión, el Tribunal Constitucional alemán puso de relieve la enorme importancia de los procedimientos administrativos (como momento de la evaluación de riesgos). ${ }^{188} \mathrm{El}$ procedimiento administrativo es una herramienta para proteger el derecho a la vida y a la integridad fisica, no sólo una herramienta de defensa, sino una herramienta activa para promover el respeto de los derechos fundamentales mediante la imposición del deber de protegerlos y promoverlos a las autoridades públicas.

\section{B. La Planificación y la Prevención de los Riesgos de Terrorismo}

El interés en las políticas públicas para la prevención de la delincuencia y el terrorismo es cada vez mayor, sobre todo en relación con el diseño del medio ambiente urbano. Diversas instituciones europeas están implicadas en los esfuerzos para prevenir la delincuencia y mejorar las condiciones de vida urbana. Un informe de la Comisión Regional sobre "La Delincuencia y la Seguridad en las Ciudades" señala: ${ }^{189}$

Es importante tener en cuenta, desde el principio de todas las obras nuevas de construcción importantes o la renovación de zonas deterioradas de la ciudad, las medidas para prevenir la violencia urbana. Esto puede lograrse mediante una estrecha colaboración entre las autoridades encargadas de la urbanización, los propietarios de edificios y las autoridades encargadas de la seguridad de la comunidad.

En una reunión el 15 de marzo de 2001, el Consejo de Justicia e Interior de la Unión Europea dio su aprobación política a las conclusiones de la conferencia de expertos europeos, la cual destacó: ${ }^{190}$

187. Ver Christian Starck, State Duties of Protection and Fundamental Rights [Deberes del Estado de Protección y de los DerechosFundamentales], 3 PotchefsTroom L.J. 1, 1-51 (2000), disponible en http://www.puk.ac.za/opencms/export/PUK/html/fakulteite/regte/per/issues/00v1star.pdf (última visita 28 de septiembre del 2011); Bundesverfassungsgericht [BVerfG] [Tribunal Federal Constitucional], 20 de diciembre de 1979, ENTSCHEIDUNGEN DES BUNDESVERWALTUNGSGERICHTS [BVERFGE] 53, 30 (1BvR 385/77) (Alem.).

188. Bundesverfassungsgericht [BVerfG] [Tribunal Federal Constitucional], 20 de diciembre de 1979, ENTSCHEIDUNGEN DES BUNDESVERWALTUNGSGERICHTS [BVERFGE] 53, 30 (1BvR 385/77) (Alem.).

189. "La delincuencia y la seguridad en las ciudades" (18 de noviembre de 1999).

190. Hacia una estrategia basada en la comprensión de prevención del delito" (Sundsvall, Suecia, 21-23 febrero del 2001). 
CPTED o $\mathrm{DCO}^{191}$ ha demostrado ser una estrategia efectiva $y$ práctica en la prevención de la delincuencia y la sensación de inseguridad, integrado por la colaboración multidisciplinaria. Las mejores prácticas a que se refiere CPTED / DCO deben ser recogidas, evaluadas y accesibles para todos los interesados. Este proceso debe utilizar un marco común y los conceptos transferibles, procesos y principios deben ser identificados.

Por último, en la Unión Europea, la conexión entre la política de vivienda y la seguridad y, en particular, el diseño urbano para la prevención de la delincuencia, ha sido recientemente destacado en el Informe de Comité de las Regiones, conocido como "Política Regional y Vivienda:"192

Los proyectos deben encajar de manera adecuada y en el espacio que les rodea. Cuando las nuevas viviendas son construidas o renovadas, las regiones y las autoridades locales deberían tomar en consideración cuestiones como el diseño, con el fin de disuadir a la delincuencia y crear zonas de calidad, desarrollo sostenible y patrimonio, además de las necesidades y aspiraciones de las comunidades locales y la mayor repercusión posible sobre la cohesión.

Este interés europeo por la prevención situacional del delito, explica el desarrollo de una norma europea para la prevención de la delincuencia por medio de la planificación urbana y el diseño arquitectónico. Como sabemos, los estándares europeos son voluntarios, complementarios, no obligatorios, entre los países, instituciones e individuos en cuanto a cómo un producto o proceso debe ser. Los principales componentes de la unidad de mercado europeo, ${ }^{193}$ son las especificaciones técnicas aprobadas por un organismo reconocido para la normalización de su aplicación repetida o continua, que puede ser internacional ${ }^{194}$ o Europeo. ${ }^{195}$

191. "CPTD" y "DCO" significa "Prevención del Delito Mediante el Disen̂o Ambiental".

192. EU Regional Committee Report on Regional and Housing Policy [Informe del Comité de las Regiones sobre Política Regional y Vivienda], n.1.8, 13 de febrero del 2007.

193. Ver generalmente European Commission on Enterprise and Industry [Comisión Europea en la Empresa y la Industria], European Standards [Estándares Europeos], http://ec.europa.eu/enterprise/ standards_policy/index_en.htm (última visita el 29 de septiembre del 2011).

194. Ver, por ejemplo, International Standardization Organization (ISO) [Organización Internacional de Estandarización], ISO 9000 Essentials, http://www.iso.org/iso/iso_catalogue/ management_and_leadership_standards/quality_management.htm (última visita el 14 de noviembre del 2011) (describiendo ISO 9000 como un estándar internacional para una administración de calidad).

195. Ver, por ejemplo, Committee of Normalization [Comité de Normalización], European EN 1522/1523 Ballistic Standards [Estándares de Balística], http://www.closefocusresearch.com/europeanen-1522-1523-ballistic-standards (última visita el 14 de noviembre del 2011) (tratando estándares para puertas y ventanas a prueba de balas). 
A mediados de 1990 , se tomó la decisión de estandarizar los procedimientos, en lugar de los productos, con el fin de ayudar a las autoridades locales y regionales, planificadores urbanos, arquitectos e ingenieros en sus esfuerzos por reducir la delincuencia, en colaboración con la policía, empresas de seguridad, las aseguradoras y asociaciones de residentes. En 1996 el Comité Europeo de Normalización creó una comisión técnica en Dinamarca para trabajar en el nuevo estándar. ${ }^{196} \mathrm{El}$ proyecto se dividió en tres Grupos de Trabajo. ${ }^{197}$ El grupo de trabajo uno estaba presidido por Francia y se concentró en los términos y definiciones. ${ }^{198}$ El grupo de trabajo dos era para la planificación urbana y estaba presidida por los Países Bajos. ${ }^{199}$ El grupo de trabajo tres estába presidido por el Reino Unido y tenía que ver con el diseño de viviendas, locales comerciales y oficinas. ${ }^{200}$

El grupo de trabajo dos ha desarrollado una norma previa europea (ENV 14383-2), aprobada por el Comité Europeo de Normalización en 2002, con una aplicación provisional de tres años, y la posibilidad de convertirse en EN. ${ }^{201}$ La ENV 14383-2 es importante, ya que es el primer esfuerzo para establecer los términos y definiciones comunes respecto a la prevención situacional del delito. ${ }^{202}$ Finalmente, en 2006 la norma EN 14383-1:2006 fue aprobada para los términos y definiciones. ${ }^{203}$ Por otra parte, durante el período de 2005 a 2007 , dos especificaciones técnicas fueron aprobadas en relación a viviendas y oficinas, así como un informe técnico. ${ }^{204}$

196. Ver generalmente EUROPEAN COMMITTEE ON NORMALIZATION [COMITÉ EUROPEO SOBRE LA NORMALIZACIÓN], A EUROPEAN STANDARD FOR THE REDUCTION OF CRIME AND FEAR OF CRIME BY Urban Planning and BuILding Design: ENV 14388-2 [Un EstánDar EuROPEO PARA la Reducción del Crimen y el Miedo del Crimen por el Planeamiento URbano y el Diseño del EDIFICIO: ENV 14388-2] (2001), disponible en http://www.securefit.org/downloads/files/ Soomeren_EuropeanStandard.pdf (última visita el 20 de octubre del 2011) [en lo sucesivo ENV 143882].

197. Id.

198. Id.

199. Id.

200. Id.

201. ENV 14388-2, supra nota 194.

202. Id.

203. EUROPEAN COMMITTEE ON NORMALIZATION [COMItÉ EUROPEO SOBRE LA Normalización], ENV 14383-1: 2006 PReVention of CRIME-URBan PlanNing AND Bullding DESIGN-PART 1: DEFINITION OF SPECIFIC TERMS [ENV 14383-1: 2006 PREVENCIÓN DEL CRIMENPlanificación Urbanística Y Diseño de Edificios-PaRTe I: DefINICIÓN de TÉrminos ESPECIFICOS] (2006).

204. Ver generalmente European Committee for Standardization [Comité Europeo para la Estandarización],

http://www.cenorm.be/CENORM/BusinessDomains/TechnicalCommitteesWorkshops/CENTechnicalC 
Entre los estados miembros de la Unión Europea, el Reino Unido y, en menor medida, Francia y España son ejemplos de países que han aplicado esta idea para el desarrollo concreto de la política de seguridad pública, la introducción de nuevas normas $\mathrm{y} / \mathrm{o}$ cambios en las prácticas administrativas. ${ }^{205}$

En el caso de Gran Bretaña, varios informes oficiales y el mismo sistema legal han incorporado esta perspectiva. ${ }^{206}$ Entre estos informes, el titulado Safer Places, elaborado en el año 2004, destaca el hecho de que la delincuencia y el miedo al delito pueden poner en peligro la cohesión social $\mathrm{y}$, en consecuencia, el desarrollo sostenible de las comunidades, insistiendo en que es más eficiente desde el punto de vista económico considerar las variables de prevención del delito en la etapa de planificación, ya que es menos costoso que el de corregir o controlar el desarrollo urbano mal diseñado. ${ }^{207}$ De la misma manera, la conexión entre la seguridad y la cohesión social se pone de relieve, lo que ha sido un elemento crucial de la agenda política británica desde 2001 , con la creación de la Unidad de Cohesión de la Comunidad, dentro del Ministerio de Interior. ${ }^{208}$

Con respecto al sistema legal, la Ley de Delitos y Desórdenes de 1998, contiene la competencia pública para la prevención del delito y el desorden, ${ }^{209}$ que en el entorno urbano ha sido definido en la Declaración de Política de Planificación I: Entrega de Desarrollo Sostenible. ${ }^{210}$ El Informe de Lugares más Seguros (Safer Places) conecta la seguridad pública con la planificación urbana y pone de relieve el vínculo entre la seguridad y la cohesión social. El informe establece la necesidad, en la etapa de planificación, de que las autoridades locales tomen en consideración las mejores prácticas establecidas en los informes oficiales

ommittees/CENTechnicalCommittees.asp?param=6306\&title $=\mathrm{CEN} \% 2 \mathrm{FTC}+325$ (última visita el 29 de septiembre del 2011).

205. Id.

206. Ver generalmente OFFICE OF THE DEPUTY PRIME MINISTER [OFICINA DEL VICEPRIMER Ministro], Safer Places: The Planning System and Crime Prevention [Lugares Mas Seguros: El Sistema de Planificación y Prevención del Delito] (Home Office, 2004), disponible en http:/www.communities.gov.uk/documents/planningandbuilding/pdf/147627.pdf (última visita el 29 de septiembre del 2011) [en lo sucesivo LUGARES MAS SEGUROS].

207. Id.

208. Id.

209. Crime and Disorder Act [Acto del Desorden y Crimen], 1998, c. 37, § 17 (U.K.).

210. Ver OfFICE OF THE DEPUTY PRIME MINISTER [OFICINA DEL VICEPRIMER MINISTRo], Planning Policy Statement I: Delivering Sustainable Development [Declaración de Política del Planeamiento I: ENTRega del Desarrollosostenible] (Controller of Her Majesty's Stationery Office, 2005), disponible en http://www.communities.gov.uk/documents/ planningandbuilding/pdf/planningpolicystatementl.pdf (última visita el 29 de septiembre del 2011) (U.K.). 
mencionados, los cuales incluyen un análisis de las diferentes experiencias en materia de acceso y movilidad en el espacio urbano (por ejemplo, acceso bien definido, evitando las escaleras aisladas, túneles, etc., diseño de vías adecuado, etc.), la estructura urbana (por ejemplo, evitar los edificios que puedan ser utilizados para comportamientos anti-sociales), la vigilancia (por ejemplo, iluminación nocturna adecuada y la instalación de circuito cerrado de televisión), "relevancia" (en referencia al diseño urbano que fomenta un sentido de comunidad, con las divisiones claramente definidas para evitar la confusión entre los espacios públicos y privados), la protección física de la propiedad privada (una perspectiva más tradicional y conocida), actividad (evitar la monofuncionalidad y la segregación urbana y fomentar el uso mixto de diferentes tipos de vivienda) y la gestión y mantenimiento (para evitar los ambientes urbanos que inciten a actos de vandalismo y aumenten el riesgo de la delincuencia). ${ }^{211}$

Estos informes y documentos legales también incluyen algunas nuevas prácticas en el área de las políticas de seguridad administrativa, como la creación de Oficiales de Enlace de Arquitectura, funcionarios especializados con experiencia en los riesgos de la delincuencia que asesoran en temas de planificación urbana, el desarrollo de la iniciativa Asegurado por el diseño, con apoyo de los principios de lucha contra el delito, mediante el uso de normas efectivas de seguridad para una amplia gama de aplicaciones. ${ }^{212}$

En el caso de Francia, los bien conocidos problemas urbanos a los que se enfrenta este país también han llevado a pensar en el diseño urbano como una herramienta para la prevención de la delincuencia y la violencia urbana, ${ }^{213}$ con similares informes oficiales sobre el tema, como en Gran Bretaña. ${ }^{214}$ Además, desde hace años, el sistema legal francés incorpora lo

211. LUGARES MAS SEGUROS, supra nota 204.

212. Ver generalmente The Offical U.K. Police Flagship Initiate Supporting the Principles of 'Designing Out Crime' [La Iniciativa de la Policia Insignia Británica Apoya los Principios del 'Diseño de la Delincuencia'], SECURED BY DESIGN (Assoc. de los Jefes de Policía), http://www.securedbydesign.com/index.aspx (última visita el 29 de septiembre del 2011).

213. Ver Alain BAUER \& XaVIER RAUFER, VIOLENCES ET INSÉCURITÉ URBAINES [VIOLENCIA E INSEGURIDAD URBANA] (PUD Paris, 9th ed., 2005).

214. Ver generalmente DIDIER PEYRAT, HABITER COHABITER: LA SECURITÉ DANS LE LOGEMENT SOCIAL [HabITAR COHABITAR: LA SEgURIDAD EN LAS VIVIENDas Sociales] (Ministry of l'équipement, des Transports et du Logement, 2001), disponible en http://lesrapports.ladocumentationfrancaise.fr/BRP/024000101/0000.pdf (última visita el 29 de septiembre del 2011) (Fr.). 
que podríamos definir como una evaluación del impacto del delito en el caso de desarrollos urbanos específico. ${ }^{215}$

En España, una modificación al reglamento urbanístico catalán se introdujo en 2006, concretando el contenido de la "Memoria Social."216 El nuevo reglamento establece que "una evaluación del impacto de la urbanización propuesta" en relación al "género, también debe formar parte de la Memoria Social, así como los grupos sociales que necesitan una atención específica, incluidos los inmigrantes y las personas mayores." Por lo que "las decisiones sobre planificación basadas en la información acerca de las realidades sociales, pueden contribuir al desarrollo de la igualdad de oportunidades entre hombres y mujeres, así como favorecer a otros grupos que necesitan protección."217 Esta evaluación debe incluir un diagnóstico de la situación y una evaluación del impacto social y de género del plan urbanístico, y tiene que justificar, entre otros aspectos, la coherencia del plan propuesto con las necesidades de hombres y mujeres y de otros grupos en relación con diversos parámetros, entre ellos "la seguridad y el uso del tejido urbano." 218

215. CODE CIVIL [C. CIV.] art. L111-3-1 (Fr.) (modificado por Ley 2007-297 de 5 de marzo del 2007, en lo queconcierne a la prevención de la delincuencia).

216. Reglamento de la Ley de Urbanismo catalana, art. 69 (B.O.E. 2005, 305) (Esp.) (la declaración de la Memoria Social es un elemento específico de los planes urbanístico ecatalanes quedeben considerar varios elementos e incluir las explicaciones para las alternativas decididas).

217. Id.

218. Id. 



\title{
DESDE QUISQUEYA HACIA BORINQUÉN: VIOLENCIA, LUCHA Y ESPERANZAS EN LAS PROPIAS VOCES A LAS MUJERES DOMINICANAS INMIGRANTES
}

\author{
Sheila I. Vélez Martinez
}

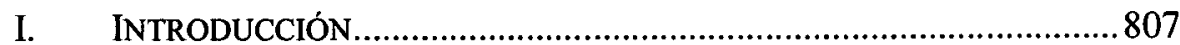

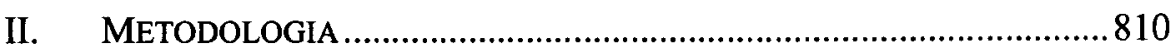

III. MIGRACIÓN DOMINICANA: FEMENINA Y TRANSNACIONAL ............814

IV. FORMANDO Y RE-FORMANDO IDENTIDADES................................8 823

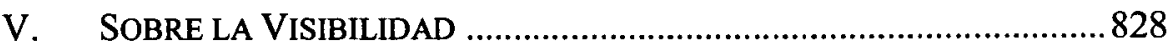

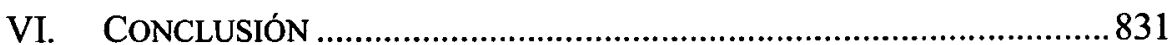

. . las migraciones no son cosas, ni los inmigrantes son mercancías. Son seres humanos con sus aspiraciones y sus necesidades, sus costumbres y rasgos culturales, sus dificultades actuales y su voluntad de futuro. Por tanto, hay que integrar el fenómeno migratorio, no sólo como variable económica, sino también como realidad humana destinada a modificar la sociedad de acogida, al modificarse a sí misma (Goytisolo y Naïr, 2000: 52-53).

\section{INTRODUCCIÓN}

De acuerdo a las Naciones Unidas más de 1.5 millones de dominicanos han emigrado de la Isla La Española, la mayoría de ellos viven en los Estados Unidos, ${ }^{2}$ lo que significa que el $17.6 \%$ de personas nacidas en la

* Profesor de Leyes Asistente de la Clínica de Derecho, Escuela de Derecho de la Universidad de Pittsburgh. Estoy en deuda con todas las mujeres inmigrantes que han poblado mi vida cotidiana durante los últimos 10 años; en particular estoy en deuda con el Centro de la Mujer Dominicana. También estoy en deuda con mi asistente de investigación Sean Ruppert y a mi eterno editor Wesley Gibbings por sus cuidados repasos y recomendaciones.

1. JUAN GOYTISOlo \& SAMI NAHIR, EL PEAJE DE LA VIDA: INTEGRACIÓN O RECHAZO DE LA EMIGRACIÓN EN ESPAÑA 226 (Aguilar ed., 2000) (España). Goytisolo and Sami Nahir . El peaje de la vida: integración o rechazo de la emigración en España 226 (Madrid: Aguilar, 2000).

2. UNITED NATIONS POPULATION FUND, DRAFT COUNTRY PROGRAM FOR THE DOMINICAN REPUBLIC [FONDO DE POBLACIÓN DE LAS NACIONES UNIDAS, PROGRAMA RPOYECTO DE PAÍS PARA LA REPUBLICA DOMINICANA] DP/FPA/DCP/ (2006), disponible en www.unfpa.org/webdav/site/global/../ dpfpa-dom-4_eng.doc (última visita 1 de noviembre del 2012). 
República Dominicana actualmente viven fuera del país. ${ }^{3}$ El número de mujeres migrantes ha aumentado constantemente desde los años $1970 .{ }^{4} \mathrm{~A}$ pesar de que el principal destino de muchas de esas mujeres es Estados Unidos y Europa, Puerto Rico es un puente de la "Visa para un Sueño." Para muchas mujeres dominicanas, Puerto Rico se presenta como un lugar de oportunidades, donde las mujeres dominicanas encuentran trabajo más fácilmente que los hombres. ${ }^{6}$ Ellas esperan que las similitudes de estilos de vida entre la República Dominicana y Puerto Rico y la existencia de una creciente comunidad, les permita adaptarse más fácilmente. ${ }^{7}$

Esta realidad genera las siguientes preguntas: ¿Quiénes son las mujeres dominicanas que emigran? ¿Cómo la experiencia migratoria da forma a sus vidas? A pesar de que existen estudios académicos relacionados con las mujeres dominicanas en Puerto Rico, ${ }^{8}$ la mayoría de la investigación se ha enfocado en las condiciones socioeconómicas de las mujeres, su participación en la fuerza de trabajo, y en su salud. En este trabajo, examino más de cerca cómo las mujeres migrantes sobrevivientes de violencia doméstica, se definen a sí mismas; cuáles son sus aspiraciones y su lugar en la sociedad. Su organización inicial alrededor del asunto de protección legal ante la violencia doméstica ha guiado hacia la realización de su ciudadanía cultural. ${ }^{9}$ El concepto de ciudadanía cultural recalca la

3. See generally The Dominican Population in the United States: Growth and Distribution [La Población Dominicana en los Estados Unidos: Crecimiento y Distribución], MIGRATION POL'Y INST. (2004).

4. Id. en $6,17$.

5. Una canción popular de merengue por el compositor y cantante Dominicano Juan Luis Guerra que fue la insporación de un documental sobre la migración dominicana a Puerto Rico. SONIA FRITZ, Visa para un sueño (Isla Films 1990).

6. Sharon Utakis \& Nelson Reynoso, No Tengo Otra Opción-Ya me voy: Stories of Separation Told by Dominican Immigrants [No Tengo Otra Opción-Ya me voy: Historias de una Separación Explicada por Inmigrantes Dominicanos], 29 ORAL HIST. F. 1 (2009).

7. Id. en 26.

8. Luisa Hernandez Angueira, Across the Mona Strait: Dominican Boat Woman in Puerto Rico, in DAUGHTERS OF CALIBAN: CARIBBEAN WOMAN IN THE TWENTIETH CENTURY 96 [A Través del Estrecho de Mona: Barco de Mujeres Dominicanas en HIJAS de CALIBAN: MUJERES Del CARIBE EN EL Siglo] (Consuelo López Springfield ed., Ind. Univ. Press 1997); MYRNa HerRera MORA, MuJERES DOMINICANAS ANTI TRUJILlistaS Y EXILIADAS EN PUERTO RICO (Isla Negra 2011); Luisa Hernandez Angueira, La Migración de Mujeres Dominicanas Hacia Puerto Rico, en Los DOMINICANOS EN Puerto Rico: Migración en la SEMI Perisferia 73 (Huracán 1990); Ramona Hernandez \& Nancy Lopez, Yola and Gender: Dominican Women in Regulated Migration, en DOMINICAN STUDIES, RESOURCES AND RESEARCH QUESTIONS 59 (CUNY 1997).

9. Ver generalmente, Anh Hua, Homing Desire, Cultural Citizenship, and Diasporic Imaginings [Deseo de Buscar, Ciudadanía Cultural, y Fantasias de la Diáspora], 12 J. INT'L WOMEN STUD. 4, 45 (2011). 
necesidad de respeto a las necesidades de los extraños. ${ }^{10}$ Se preocupa por "quién tiene la necesidad de ser visible, de ser escuchado, y depertenecer." En el contexto de las mujeres inmigrantes dominicanas, las respuestas a esas preguntas han sido construidas desde afuera. ${ }^{12}$

En esta presentación, me adentro en la discusión de las experiencias de las mujeres dominicanas en Puerto Rico usando sus propias voces, voces que narran la construcción y deconstrucción de sus identidades. Estas mujeres han vivido a través de aterradoras y frecuentemente deplorables experiencias de violencia y de privación de derechos, pero además han tenido historias y experiencias maravillosas durante el camino. Estas mujeres, en más de una forma, "retan el discurso dominante relacionado con la sumisión de las mujeres, la intuición, y la dependencia vis-a-vis los hombres."13

Propongo que mientras las mujeres inmigrantes han puesto sus vidas en juego por sus familias y por ellas mismas, no son pasivas y son mucho más que meramente víctimas. La experiencia de migración para esas mujeres pudo haber sido marcada por la violencia doméstica y la explotación, pero además les ha dado un sentido de liderato e independencia. ${ }^{14}$ Las voces activas de las mujeres del Centro de la Mujer Dominicana juegan un papel importante en llenar el vacío de información relacionada con las experiencias migratorias de las mujeres dominicanas en Puerto Rico. ${ }^{15}$

10. Id.

11. Solen Sanli, Public Sphere and the Symbolic Power: Woman's Voice as a Case of Cultural Citizenship [Esfera Pública y el Poder Simbólico: La Voz de las Mujeres Como un Caso de la Ciudadania Cultural], 5 CULTURAL SOC. 281, 283-89 (2011).

12. Suzanna Oboler, Racializing Latinos in the United States: Toward a New Research Paradigm [Clasificando Latinos en los Estados Unidos: Hacia un Nuevo Paradigma de la Investigación], en IDENTITIES ON THE MOVE: TRANSNATIONAL PROCESSES IN NORTH AMERICA AND the Caribbean Basin [Identidades en Movimiento: Procesos Transnacionales en Norte AMÉRICA Y El CARIBE DE BASIN] 45 (Univ. of Tex. Press, 1999); ver también Jorge Duany, Racializing Ethnicity in the Spanish Speaking Caribbean: A Comparison of Haitians in the Dominican Republic and Dominicans in Puerto Rico [Clasificando Étnias en el Caribe de Habla Hispánica: Una Comparación de Haitianos en la República Dominicana y Dominicanos en Puerto Rico], en HOW THE UNited States RaCializes latinos [Cómo los Estados Unidos Clasifica a los latiNos de ACUERDO A SU RAZA] 214 (Paradigm 2011).

13. Hernandez Angueira, supra nota 8, en 96, 97.

14. United for a Life without Violence! [Unidos por una Vida sin Violencia!], CENTRO DE LA MUJER DOMINICANA (2012), disponible en http://cemud.org/english-version.php (última visita el 23 de marzo del 2012).

15. Id. 


\section{Metodologia}

Me propongo presentar las voces y las experiencias de estas mujeres con la colaboración del Centro de la Mujer Dominicana en San Juan. El Centro es la única organización sin fines lucrativos creada para contribuir en apoderar a las mujeres Dominicanas y sus familias en Puerto Rico. ${ }^{16}$ Es la única organización de ese tipo creada por mujeres inmigrantes, y sirviendo a mujeres inmigrantes. El Centro está comprometido en organizar a las mujeres para participar activamente en la eliminación de la desigualdad de género y la promoción de la justicia social. ${ }^{17} \mathrm{Ha}$ sido inicialmente organizado alrededor de asuntos de violencia de género y protección legal de las mujeres inmigrantes disponibles bajo la Ley deViolencia en Contra de la Mujer (VAWA por sus siglas en inglés). ${ }^{18} \mathrm{La}$ organización comunitaria alrededor de VAWA ha permitido al Centro forjar una comunidad activa de mujeres migrantes que son protagonistas de sus propias vidas. ${ }^{19}$ Como dice Romelinda Grullón, la directora del Centro: "las cogemos por lo legal y se quedan para todo lo demás."

Las historias contadas por las mujeres inmigrantes son el centro de este trabajo, y las historias importan. Como señala Richard Kearney "la existencia humana es inherentemente contada. La vida está preñada con historias." "21 Para él, las historias nos permiten rehacer el mundo a la luz de lo que es fundamental para nosotros. ${ }^{22}$ Nos permite entender la vida como

16. Firuzeh Shokooh Valle, Espacio para Respirar Solidaridad, PRIMERA HORA (17 de octubre del 2007), disponible en http://www.primerahora.com/espaciopararespirarsolidaridad119509.html (última visita el 23 de marzo del 2012).

17. Id.

18. Ver The Violence Against Women Act [Ley Contra la Violencia de Género], Pub. L. No. 103-322, §§ 40121, 40211, 40241, 108 Stat. 1796 (1994); The Violence Against Women Act of 2000 [Ley Contra la Violencia de Género del 2000], Pub. L. No. 106-386, 114 Stat. 1464, (2000); The Keeping Children and Families Safe Act of 2003 [Ley Para Mantener a los Niños y las Familias Seguros del 2003], Pub. L. No. 108-36, 117 Stat. 800-831 (2003); The Protect Act [Ley de Protección], Pub. L. No. 108-21, 117 Stat. 650 (2003); The Violence Against Women and Department of Justice Reauthorization Act of 2005 [Ley de Violencia de Género y de Reautorización del Departamento de Justicia], Pub. L. No. 109-162, 119 Stat. 2960 (2006).

19. The Violence Against Women Act of 2000 [Ley Contra la Violencia de Género del 2000], Pub. L. No. 106-386, 114 Stat. 1464, (2000).

20. Romelinda Grullón, Dir., Centro de la Mujer Dominicana, presentación en LatCrit SNX 2011: Particularidades de las Mujeres Inmigrantes Víctimas de Violencia Doméstica en Puerto Rico (13 de mayo del 2011).

21. RICHARD KEARNEY, ON STORIES (THINKING IN ACTION) [EN HiSTÓRIAS (PENSANDo EN ACCIÓN)] 130 (Routledge 2002).

22. Id. en 132 . 
se relata. ${ }^{23}$ También señala que las historias tienen un poder catalítico, ellas nos cambian transportándonos a otros momentos y lugares donde podemos experimentar cosas de otro modo. ${ }^{24}$ Pero probablemente lo más importante es la dimensión ética de las historias contadas. Para Kearney, el punto más básico para recordar es "que las historias hacen posible un compartir ético de un mundo común con los otros." 25 La erudición legal en general y la erudición de la teoría Lat Crit en particular, han reconocido la importancia de las historias en la manera como pensamos sobre la enseñanza y la práctica del derecho. ${ }^{26} \mathrm{El}$ objetivo de contar historias está además vinculado con la promoción y el entendimiento de las dinámicas de opresión fundamentadas en raza, género, orientación sexual y otras marginaciones. ${ }^{27}$

En el proceso de la investigación, profundicé en la Investigación Participativa para la Acción. La Investigación Participativa para la Acción (PAR por sus siglas en inglés) es una forma de recopilar información que respeta, se centra y reflexiona más directamente sobre las experiencias de la gente afectada por situaciones de la comunidad a la que pertenecen. ${ }^{28}$ Más que un método, es una filosofia, y además es una forma de construir y fortalecer las comunidades y nuestro entendimiento de sí mismo, de los otros, y de nuestras relaciones. ${ }^{29}$ PAR se distingue por tres facetas principales:

1. Es un proceso interactivo para llevar a cabo investigación que incluya reflexión y acción

2. Integra a los miembros de la comunidad y las partes interesadas en el proceso de la investigación; y

23. Id.

24. Id. en 137.

25. Id. en 150.

26. Ver generalmente Berta Hernandez-Truyol et al., Beyond the First Decade: A ForwardLooking History of LatCrit Theory, Community, and Practice, 17 BERKELEY LA RAZA L.J. 169 (2006); Alfredo Mirande, Alfredo's Caribbean Adventure: Lat Crit Theory, Narratives, and the Politics of Exclusion, 26 CHICANO-LATINO L. REV. 207, 206 (2006).

27. Jane C. Murphy, Lawyering for Social Change: The Power of the Narrative in Domestic Violence Law Reform [Abogar para un Cambio Social: El Poder de la Narrativa en la Reforma de la Ley de Violencia Doméstica], 21 HofSTRA L. REV. 1243, 1245 (1993).

28. Ann Macaulay et al., Participatory Action Research Maximises Community and Lay Involvement, 319 BRIT. MED. J. 774, 774 (1999).

29. Id. 
3. Utiliza los hallazgos para promover cambios positivos en la comunidad. $^{30}$

Puede ser un instrumento poderoso para el alcance, la construcción de la base y la organización comunitaria. Puede, además, ayudar a unir a la gente para construir movimientos de cambio social. ${ }^{31}$ Particularmente en el contexto de género, PAR abre la puerta a las mujeres para investigar sus propias vidas. $^{32}$

Las citas de las mujeres que aparecen en este trabajo son el resultado de entrevistas a profundidad que son parte de un proyecto de investigación amplio del Centro. Algunas de las mujeres fueron entrevistadas en el Centro y ellas me hicieron disponibles sus declaraciones. Todas las mujeres entrevistadas tienen historial de violencia doméstica. ${ }^{33}$ Me reuní con ellas por varias horas en el Centro, en su residencia familiar, y en parques. Algunas de ellas las he conocido durante varios años debido a mi colaboración con el Centro. Otras, las conocí por primera vez para la entrevista. Como parte de la investigación, se me permitió participar en la proyección de la película América, de la cineasta Sonia Fritz, que se proyectaba en el Centro. La película está basada en la novela Sueño Americano de Esmeralda Santiago. ${ }^{34}$ Es la historia de una mujer puertorriqueña joven de la Isla de Vieques, que es abusada por el esposo y se escapa a la Ciudad de Nueva York. ${ }^{35}$ América, el personaje principal era abusada física y emocionalmente y aguanta el secuestro de su hija por el

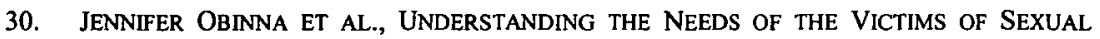
Assault IN THE Deaf COMMUNITY 24 [ENTENDIENDO las NeCESIDADES de Las Víctimas de ACoso SEXUAL EN LA COMUNIDAD SORDA] (2006), disponible en https:/www.ncjrs.gov/ pdffiles1/nij/grants/212867.pdf (última visita el 19 de marzo del 2012); M. O'Neill, P. Woods, \& M. Webster, New Arrivals: Participatory Action Research, Imagined Communities and Social Justice [Nuevas Llegadas: Investigación de Acción Participativa, Comunidades Imaginadas y Justicia Social] 32 J. SOC. JUST. 75,75 (2004).

31. OBINNA, supra nota 30, en 24.

32. Ver generalmente Alice MACINTYRE, PaRTICIPATORY ACTION RESEARCH 19 [INVESTIGaCión de ACCIÓN PARTICIPATIVA] (Sage Publications 2008); Caitlin Cahill, The Personal is Political: Developing New Subjectivities through Participatory Action Research [El Personal es Politico: Desarrollando Nuevas Subjetividades Mediante la Investigación de Acción Participativa], 14 GENDER, Place \& CULTURE 267, 268 (2007)

33. Los nombres de las mujeres han sidomodificados para proteger sus identidades con la excepción de Gregoria Grullart, quien ha insistido en reveler la suya. Las cuotas están en Español y traducidas liberalmente por el autor al Inglés.

34. Ver generalmente Esmeralda Santiago, AmÉrica's Dream [Sueño americano] (Harper Collins Publishers 1996).

35. Id. 
abusador. ${ }^{36}$ Él mantiene amenazas constantes, incluso le sigue hasta Nueva York. ${ }^{37}$ No obstante, con el apoyo de la familia y el de otras inmigrantes Latinas, América encuentra consuelo y esperanza en su nueva vida como niñera en Nueva York. ${ }^{38}$ Afortunadamente, y a diferencia de la mayoría de los casos, el abusador fue arrestado y América pudo descubrir por primera vez la posibilidad de vivir su sueño. ${ }^{39}$ El propósito de la proyección era promover una discusión abierta de las mujeres relacionada con las experiencias y el apoderamiento de sus esperanzas y sueños. ${ }^{40}$ La noche se transformó en una montaña rusa de emociones y de perspicaces comentarios, llenos de humor. ${ }^{41}$ El humor fue una de las características destacados de todas las mujeres entrevistadas. ${ }^{42}$

Nadie describe mejor la experiencia que Gregoria Grullart: "Lo que pasa es que yo vivo con la fuente de la juventud, como yo vivo muerta de risa, y no le he dado mente a los fracasos que me han pasado, yo vivo feliz." ${ }^{, 43}$ Gregoria es una sobreviviente de abuso doméstico y cáncer. ${ }^{44}$ Antes de dejar la República Dominicana, le hicieron una biopsia en su brazo que parecía ser negativo. ${ }^{45}$ Tan pronto le dieron los resultados se fue del hospital sin autorización médica, se montó en una yola (bote de madera pequeño) y se fue rumbo a Puerto Rico. ${ }^{46}$ Llegó a Puerto Rico con su hermano, su primo, y su cuñada en una yola que tenía alrededor de 150 personas a bordo. ${ }^{47}$ Después de llegar a Puerto Rico le diagnosticaron cáncer en el brazo. ${ }^{48}$ Como resultado, el brazo tuvo que ser amputado. ${ }^{49}$ Gregoria, sin embargo, no se ha arrepentido y dice que lo haría de nuevo. ${ }^{50}$

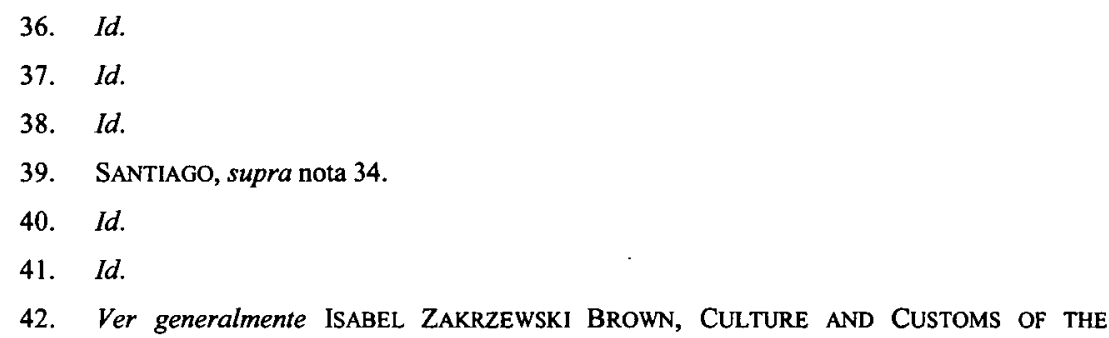
DOMINICAN REPUBlic [CULTURA y COSTUMBRES DE LA RePúBliCa DominiCANA] 44 (Greenwood Press 1999).

43. Entrevista con Gregoria Grullart ( 13 y 14 de abril del 2011) [en lo sucesivo Entrevista con Grullart].
44. Id.
45. Id.
46. Id.
47. Id.
48. Entrevista con Grullart, supra nota 43.
49. Id.
50. Id. 
De hecho, está convencida de que el cáncer la hubiera matado si se quedaba en la República Dominicana. ${ }^{51}$ Gregoria llega a decir: "Sufrí mucho por lo del brazo, pero después he meditado mucho. Lavo, cocino, pelo plátanos, trabajo en construcción, limpio mi casa y he tenido marido." ${ }^{2 p}$

\section{MIGRACIÓN DOMINICANA: FEMENINA Y TRANSNACIONAL}

Los procesos de la migración dominicana son análogos a los del resto del Caribe, como se puede ver como parte de la tradición regional. Sagás y Molina han caracterizado la migración dominicana de los últimos 50 años como "una migración masiva de proporción diaspórica." 53 Rose Mary Allen ha analizado el trabajo de diferentes sociólogos, incluyendo Gordon K. Lewis, para exponer cómo las instituciones a través del Caribe facilitan y perpetúan la migración. También habla sobre el Caribe como sociedad frontera, donde la gente está "constantemente yéndose en busca de una mejor vida." 54

Los procesos migratorios de la República Dominicana son transnacionales y cada vez más femeninos. A la vez que el número de hombres y mujeres migrantes ha aumentado, también lo ha hecho la proporción de mujeres (de cuarenta y siete por ciento en 1960 a cuarenta y nueve por ciento actualmente, con algunas diferencias entre países). ${ }^{55}$ Sin embargo, lo que realmente ha cambiado durante los pasados cuarenta años es el aumento de mujeres que han migrado en busca de trabajo, en lugar de viajar con sus esposos o para reunirse con ellos. ${ }^{56}$ Además, se debe

51. Id.

52. Id.

53. ERnesto Sagas \& Sintia Molina, Dominican migration transnational Perspectives [Perspectivas de la Migración Transnacional Dominicana] 1 (Univ. Press Fla. 2004).

54. Rose Mary Allen, Regionalization of Identity in Curacao: Migration and Diaspora [Regionalización de la Identidad en Curacao: Migración y Diáspora], en CARIBBEAN Transnationalism Migration, Pluralization and Social Cohesion [Migración del Transnacionalismo CaribeÑo, PluRalización y Cohesión Social] 79 (Lexington Books 2006).

55. Ver generalmente ROBERTO BERGALLI, FLUJOS MIGRATORIOS Y SU (DES)CONTROL. PUNTOS DE VISTA PLURIDISCIPLINARIOS (Anthropos Barcelona 2006).

56. U.N. INT'L RES. \& TRAINING INST. FOR THE ADVANCEMENT OF WOMEN [INSTITUTO INTERNACIONAL DE INVESTIGACIONES Y CAPACITACIÓN DE LAS NACIONES UNIDAS PARA LA PROMOCIÓN DE LA MUJER], Gender Remittances and Development: Feminization of Migration [Las Remesas de Género y el Desarrollo: Feminización de la Migración] 1 (2007), disponible en http://download-document/615-working-paper-1-feminization-of-migration.html (última visita el $23 \mathrm{de}$ marzo del 2012). 
observar que estas mujeres migrantes son más jóvenes que las que han emigrado anteriormente. ${ }^{57}$

De acuerdo a la División de Población de las Naciones Unidas, en el 1990 las mujeres inmigrantes de Latinoamérica y el Caribe comenzaron a ser las primeras en alcanzar igualdad con los migrantes varones, en el mundo desarrollado. ${ }^{58}$ En el año 2000 las mujeres migrantes constituyeron el $50.1 \%$ de la migración total de la región. ${ }^{59}$

Este patrón de migración es consistente con el crecimiento migrante en los Estados Unidos. ${ }^{60}$ Por ejemplo, más mujeres se hicieron Residentes Permanentes Legales, Ciudadanas Naturalizadas y fueron adoptadas en los Estados Unidos durante el 2009. ${ }^{61}$ Interesantemente, el estimado de la población migrante no autorizada en los Estados Unidos indica que las mujeres forman el cincuenta y dos por ciento del grupo de cuarenta y cinco años para arriba. ${ }^{62}$ Se estima que hay 1.3 millones de niños indocumentados viviendo en los Estados Unidos. ${ }^{63}$ De esos, más de 600,000 inmigrantes son niñas, representando el trece por ciento del total femenino de la población de inmigrante no autorizadas en los Estados Unidos. ${ }^{64}$

Este es un ejemplo de la feminización de la inmigración. ${ }^{65}$ Sassen ha llamado este fenómeno la "feminización de la sobrevivencia" porque la

57. BERGALLI, supra nota 55.

58. Zlotnik, Hania, The Global Dimension of Female Migration, Migration Policy Institute (March 2003). See also Glenda Labadie-Jackson Reflections On Domestic Work and The Feminization of Migration 31 Campbell L. Rev. 67, 73 (2008).

59. Ver id; ver también Glenda Labadie-Jackson, Reflections on Domestic Work and the Feminization of Migration [Reflexiones Sobre el Trabajo Doméstico y la Feminización de la Migración], 31 CAMPBELL L. REV. 67, 73 (2008).

60. Dep't of Homeland Security, OfF. OF IMMigr. Stat., 2009 Yearbook of IMMIGRATION STATISTICS [DEPARTAMENTO DE SEGURIDAD NACIONAL, OfICINA DE ESTADístICAS DE INMIGRACIÓN, ANUARIO DE ESTADISTICAS DE LA INMIGRACIÓN] 54 (2009) [en lo sucesivo ANUARIO DEL 2009].

61. Id.

62. DEP'T OF HOMELAND SECURITY, OFF. OF IMMIGR. STAT. [DEPARTAMENTO DE SEGURIDAD NACIONAL, Oficina dE ESTADÍSTICAS DE INMIGRACIÓN], Estimates OF THE UNAUTHORIZED IMMIGRANT POPULATION RESIDING IN THE UNITED STATES: JANUARY 2009 [ESTIMACIONES DE LA POBLACIÓN INMIGRANTE RESIDENTE EN ESTADOS UNIDOS: ENERO 2009], en 5 (2010).

63. Id.

64. Alexandra Dobrowlsky \& Evangelina Tatsoglou, Crossing Boundaries and Making Connections [Cruzando Fronteras y Haciendo Conexiones], en WOMEN, MIGRATION AND CITIZENSHIP [MUJERES, Migración Y CIUdAdANÍA] 1, 3 (Ashgate 2006).

65. Id. 
tarea de garantizar la sobrevivencia de la familia y la comunidad, al aumentar cada vez más, ha caído en los hombros de las mujeres. ${ }^{66}$

En el contexto del Caribe, este componente transnacional es además destacado por la existencia de la migración dentro del Caribe. ${ }^{67}$ Como Allen señala, este movimiento a través de la región ha sido una de las estrategias de sobrevivencias principales utilizadas por las clases pobres en esas sociedades. ${ }^{68}$

Más de un millón de dominicanos han obtenido su residencia legal permanente en los Estados Unidos durante los últimos veinte años. ${ }^{69}$ Más de dos millones han conseguido visas de no-inmigrantes para viajar a los Estados Unidos durante los últimos diez años. ${ }^{70}$ Pero solo alrededor de 10,000 visas relacionadas con trabajo han sido otorgadas durante el mismo período de tiempo. ${ }^{71}$ De esos cerca de 200,000 se han hecho ciudadanos de los Estados Unidos. ${ }^{72}$

Los dominicanos constituyen el tres por ciento del total de la población hispana en los Estados Unidos. ${ }^{73}$ Esta población creció por ochenta y cinco por ciento, aumentando de 765,000 en el 2000 a 1.4 millones en el 2010. ${ }^{74}$ Los dominicanos constituyen el grupo de inmigrantes más grande en Puerto Rico, la población dominicana aumentó veintinueve por ciento o 12,000 personas, desde el Censo del $2000 .^{75}$ Los dominicanos constituyen el dos por ciento de toda la población hispana en la isla. ${ }^{76}$ Se estima que hay diecinueve millones de mujeres inmigrantes en los Estados Unidos. ${ }^{77}$ Las mujeres dominicanas constituyen el dos por

66. Saskia Sassen, Counter Geographies of Globalization and the Feminization of Survival [Geografias Contrarias a la Globalización y la Feminización de la Supervivencia], 53 J. INT'L AFF. 503, 506 (2000).

67. Allen, supra nota 54, en 81 .

68. Id.

69. ANUARIO DEL 2009, supra nota 60 en 8.

70. Id.

71. Id.

72. U.S. CEnsus Bureau, U.S. Dep'T of COMM., The Hispanic Population: 2010 [LA POBLACIÓN HISPANA: 2010], 4 (2011) disponible en http://www.census.gov/prod/ cen2010/briefs/c2010br-04.pdf (last visited Mar. 28, 2012) [en lo sucesivo LA POBLACión HISPANA]

73. Id. en 3 .

74. Id. en 11 .

75. Id.

76. Id.

77. See U.S. Census Bureau, U.S. DeP'T OF COMM., AMERICAN COMMUNITy SuRVEy

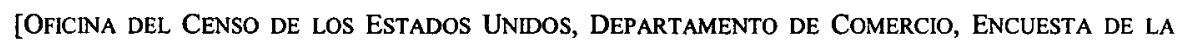
COMUNIDAD AMERICANA] (2008)[en lo sucesivo ENCUESTA DE LA COMUNIDAD AMERICANA]. 
ciento de todas las mujeres inmigrantes en los Estados Unidos. ${ }^{78}$ Las mujeres inmigrantes superan el número de hombres inmigrantes por casi $20 \%{ }^{79}$ Esta tendencia de migración femenina a Puerto Rico ha sido consistente durante los últimos veinte años. ${ }^{80}$ No existen datos definitivos del censo relacionados con el número de inmigrantes en Puerto Rico. ${ }^{81}$ Sin embargo, sabemos que más mujeres dominicanas inmigran legalmente como resultado de peticiones de la familia y que más hombres son interceptados en el mar por la Guardia Costera de los Estados Unidos. ${ }^{82}$

Esa yola, esa yola era bien grande. Era tremenda yola con dos motores de 150 cada uno, con mucha gasolina, cómoda, salami de todo y con bolsitas de agua muchas bolsitas de aquí. Era como de 150 personas. Era como una casa de alta. [. . .] Nos tardamos veinticuatro horas en llegar porque nos perdimos en el mar, porque el tipo no sabía supuestamente la ruta. ${ }^{83}$

Cuarenta años atrás, no tenía planes de tomar la yola para ir a Puerto Rico, pero su hermano sí. ${ }^{84}$ El había estado trabajando durante algún tiempo con contrabandistas antes de decidir que debía dejar el país. ${ }^{85}$ Antes de que le llegara el tiempo para partir, cambió de planes y decidió que Gracia debía ir primero porque su vida era muy dificil. ${ }^{86}$ Todavía quería ayudar al jefe de los contrabandistas, ya que había cientos de personas en turno para ir a Puerto Rico. ${ }^{87}$ De cualquier manera, cuando llegó el tiempo de partir, a ella le dio miedo y trató de quedarse con él. ${ }^{88}$ Desafortunadamente, ella tenía la única brújula. ${ }^{89}$ La yola regresó a recoger a la mujer de la brújula. ${ }^{90}$ Ella rehusó montarse, pero como tenía la brújula la halaron en contra de sus deseos. ${ }^{91}$ Agarró a su hermano y su viaje a

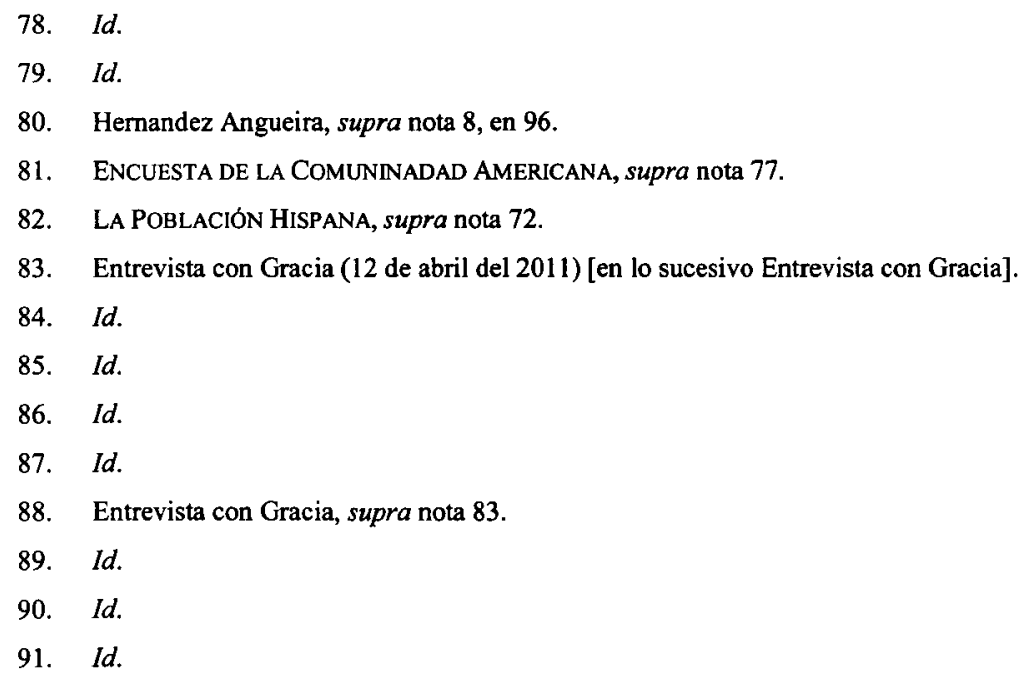


Puerto Rico comenzó. ${ }^{92}$ Ella estaba sorprendida por el hecho de que ellos la necesitaban y que sin ella estaban perdidos. ${ }^{93}$ Gracia ve la anécdota como una metáfora de su vida. ${ }^{94}$ Se siente con suerte a pesar de todo y está convencida que ha hecho la diferencia en su propia vida, en la vida de los hijos que dejó atrás e incluso en la vida del abusador. ${ }^{95}$ Ahora se ve a sí misma como la mujer de la brújula. ${ }^{96}$

El Mar Caribeño es un continuo de bordes difusos que todos nosotros filtramos hacia el sueño de una nueva vida. Los haitianos van a la República Dominicana, los dominicanos a Puerto Rico y los puertorriqueños a Nueva York. Este intercambio es construido dentro de un contexto multicultural creado por la corriente de migración y las relaciones transnacionales. Relaciones que han existido de una forma o de otra por algún tiempo pero que ahora son más evidentes y más complejas.

Michael Peter Smith define estas relaciones transnacionales como:

Un marcador de comunicación de circuitos transnacionales entrecruzados y prácticas transversales locales y translocales que se unen en determinados lugares y tiempos particulares y se inscriben en el debate político, la construcción social del poder, y la formación de individuos, grupos, identidades nacionales $y$ transnacionales y sus campos correspondientes de diferencias. ${ }^{97}$

Hablando sobre Appadurai, Glover también argumenta que: “esas fuerzas poderosas han desencadenado la imaginación popular y generado nuevas posibilidades sociales y culturales que transcienden los horizontes previos situados por sus países y comunidades de origen." ${ }^{\text {"98 }}$ No todos estos desarrollos son emancipatorios, pero la naturaleza cambiante significa que las posibilidades emancipatorias y democráticas no deben descartarse. ${ }^{99}$

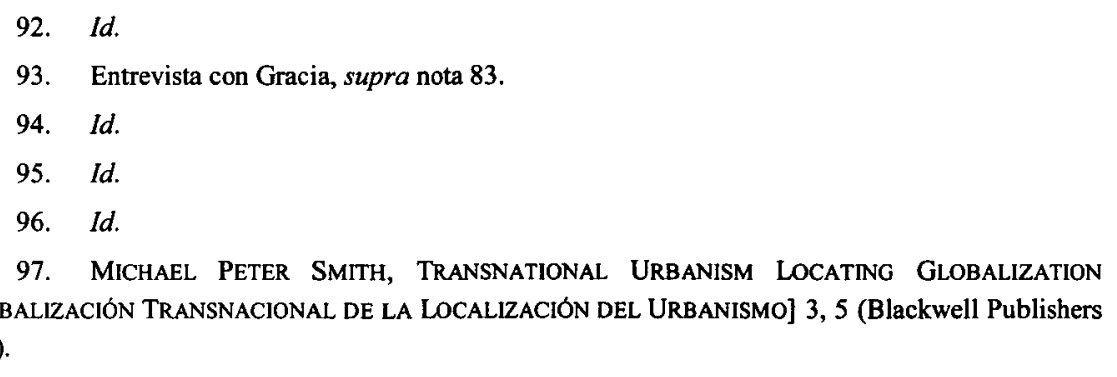

98. See David Glover, Preface to Silvia Pilar Castro \& Maria Isabel Borrero,

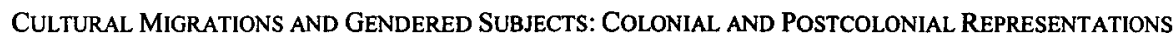
of the Female Body [Prefacio $a$ Silvia Pilar Castro \& Maria Isabel Borrero, Migraciones Culturales y temas de Género: Representaciones Coloniales y postcoloniales del CUERPO FEMENINO] (2011).

99. Id. 
Todas las mujeres entrevistadas tienen comunicación casi diaria con sus hijos y el resto de la familia inmediata que dejaron atrás. ${ }^{100}$ Aún desde otra nación se las arreglan para estar presentes y cuidar de su familia. ${ }^{101}$ Como dijo Gregoria:

Yo los llamo y los llamo casi todos los días, pero la verdad eso es algo como que uno no encuentra que se los tiene como uno quiere. Por ejemplo cuando yo llamo, ya son las nueve de la noche y todavía andan en la calle, eso me deprime porque yo se que lo hacen porque yo no estoy alli. ${ }^{102}$

Interesantemente, de acuerdo al profesor Araujo Dawson las prácticas transnacionales de los Dominicanos facilitan la conexión con su país de origen y sus prácticas culturales de forma que sirven de protección del estrés y la salud mental, consecuencias de la discriminación que frecuentemente las latinas tienen que afrontar. ${ }^{103}$ Ella añade que "esta conexión cultural les puede ayudar a manejar la discriminación." 104 Específicamente encontró en su estudio que mientras más cerca las mujeres dominicanas estaban de su cultura y tenían menores niveles de aculturación en los Estados Unidos, más protegidas estaban del impacto negativo de las experiencias discriminatorias. ${ }^{105}$

Las mujeres entrevistadas también supervisan cómo se usan sus remesas. ${ }^{106}$ Las remesas son usualmente el recurso de ingreso de los hijos y la familia inmediata. ${ }^{107}$ Las mujeres necesitan saber que las familias están cuidando del dinero que ellas envían. "Cuando le mandaba dinero a mis hijos se los mandaba a mi mamá, pero después se los mandaba al papá. Pero le ponía una nota y llamaba de una vez al más grande y le decía: papi te mandé tanto, para ti y para tu hermano."108

100. Entrevista con Grullart, supra nota 43.

101. Id.

102. Id.

103. Beverly Araújo Dawson, Discrimination, Stress, and Acculturation Among Dominican Immigrant Women [Discriminación, Estrés, y Aculturación Entre las Mujeres Inmigrantes Dominicanas], 31 HISP. J. BEHAV. SCI. 96, 105 (2009).

104. Id. en 106.

105. Id. en 105 .

106. Entrevista con Gracia, supra nota 83.

107. Id.

108. Id. 
Las mujeres entrevistadas envían dinero regularmente a su familia. ${ }^{109}$ Para muchos eso el centro de su existencia. ${ }^{110}$ Dina, la de mayor edad entrevistada, es una de esas mujeres. ${ }^{111}$ Una persona extraordinaria que vino a Puerto Rico ocho años atrás. ${ }^{112}$ Emigró después que se casó con un hombre puertorriqueño que conoció en la República Dominicana. ${ }^{113}$ Ella tiene una hija producto de una violación que ocurrió cuando tenía quince años. ${ }^{114}$ En Puerto Rico estuvo casada durante dos años hasta que no pudo tolerar más el abuso. ${ }^{15}$ Ahora es una empleada de servicio doméstico y vive muy bien. ${ }^{116}$ Tiene más ofertas de trabajo de las que puede aceptar $\mathrm{y}$ ha estado trabajando con la misma familia durante los últimos cinco años. ${ }^{117}$ Ella envía casi la mitad de su ingreso a su casa. "Tengo que cuidar a la viejita y a mi hija y a mis nietos, si yo no le mando, quién le va a mandar." 118 Ella ha construido una casa grande para su mamá y su hija, y paga todos los gastos médicos y de la escuela. ${ }^{119}$ Ella es una de las que han tenido suerte porque es Residente Legal Permanente y puede viajar a la casa una vez al año para la Navidad. ${ }^{120}$

De acuerdo al Comisionado de Asuntos Financieros de Puerto Rico, las remesas de Puerto Rico a la República Dominicana en el 2010, alcanzaron la cifra de $\$ 156.6$ millones. ${ }^{121}$ Esto representa veintiocho por ciento de todas las remesas enviadas desde Puerto Rico. ${ }^{122}$ La cantidad

109. Entrevista con Dina (14 de abril del 2010) (note que Dina no es el nombre verdadero de la mujer entrevistada. Su nombre ha sido cambiado para proteger su identidad)[en lo sucesivo Entrevista con Dina].

110. Id.

111. Id.

112. Id.

113. Id.

114. Entrevista con Dina, supra nota 109.

115. Id.

116. Id.

117. Id.

118. Id.

119. Entrevista con Dina, supra nota 109.

120. Id.

121. Oficina del Comisionado de instituciones financieras de Puerto Rico, TRANSFERENCIAS POR DESTino PARA El AÑo TERMinado, (P.R. 2011).

122. Id. 
promedio fue $\$ 154.02$, lo que significa que se llevaron a cabo más de un millón de transacciones en un solo año. ${ }^{123}$

Las remesas de Puerto Rico representan el nueve por ciento de todas las remesas que se reciben en la República Dominicana. ${ }^{124}$ Las mujeres dominicanas envían más remesas que los hombres, en términos absolutos y relativos. ${ }^{125}$ Aunque, las mujeres dominicanas constituyen el 52.5 por ciento de los inmigrantes a los Estados Unidos, son responsables del cincuenta y ocho por ciento de las remesas enviadas a este país. ${ }^{126}$ Ellas también envían más dinero directamente a otras mujeres en la República Dominicana. ${ }^{127}$ El informe del Inter-American Development Bank Multilateral Investment Fund estimó que tanto los que envían como los que reciben remesas son generalmente personas de bajo nivel de educación, y sobre el $60 \%$ de las mujeres son jefas de familia. ${ }^{128}$

Dado que el promedio de ingreso de las mujeres es más bajo que el de los hombres a través de todos los destinos de migración, el porcentaje más alto enviado constituye un alto nivel de sacrificio personal de la mujer. ${ }^{129}$ Con el propósito de enviar un alto porcentage de su ingreso, las mujeres frecuentemente disminuyen sus propios gastos de salud, educación, nutrición y tiempo de ocio. ${ }^{130}$ La profesora Wayland argumenta que la experiencia transnacional ha demostrado que es provechosa y apodera a las mujeres, "según sus condiciones mejoran también mejoran las remesas para ayudar a pagar las matriculas de los hijos, necesidades básicas, gastos

123. Jorge Duany, To Send or not to Send [Enviar o no Enviar], en CONTINENTAL DIVIDES: INTERNATIONAL MIGRATION IN THE AMERICAS [DIVISIÓN CONTINENTAL: MIGRACIÓN INTERNACIONAL EN LAS AMÉRICAS] 205, 207 (Sage 2010).

124. Id. en 205, 207; U.N. INT'L RES. \& TRANNING INST. FOR THE ADVANCEMENT OF WOMEN [INSTITUTO INTERNACIONAL DE INVESTIGACIONES Y CAPACITACIÓN DE LAS NACIONES UNIDAS PARA LA PROMOCIÓN DE LA MUJER], Women Gender and Development: The Case of Women Migrants from Vicente Noble, Dominican Republic [El Género de Mujeres y el Desarrollo: El Caso de las Mujeres Migrantes de Vicente Noble, República Dominicana] 131 (2006) [en lo sucesivo El Género de Mujeres y el Desarrollo].

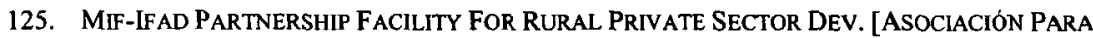
El Desarrollo del Sector Privado RuRal], Remittances and RURAL deVElopment in the DOMINICAN REPUBLIC [LAS REMESAS Y EL DESARROLLO RURAL EN LA REPÚBLICA DOMINICANA] 2 (Enero 2005).

126. Id.

127. Id.

128. Id.

129. Id.

130. El Género de Mujeres y el Desarrollo, supra nota 124, en 29. 
médicos, $\mathrm{y}$ muchas veces ayudan a crear oportunidades de trabajos adicionales para los que se quedaron atrás."131

Los académicos han considerado las remesas como uno de los más fuertes vínculos transnacionales entre los que las envían y los que las reciben. Como plantea Duany, las remesas pueden ser consideradas como una forma clásica de transnacionalismo desde abajo. ${ }^{132}$ Él sostiene que "esta transferencia masiva de recursos son incrustados en 'far flung webs' o redes lejanas de solidaridad y reciprocidad entre los parientes y amigos que atraviesan las fronteras nacionales." 133

Algunas de las mujeres han experimentado relaciones transnacionales, aún antes de emigrar. ${ }^{134}$ Dina, por ejemplo, conoció a su esposo cuando él fue con un amigo a visitar la República Dominicana. ${ }^{135}$ Desde entonces mantiene relaciones de larga distancia por teléfono. ${ }^{136}$ Muchas de las mujeres entrevistadas señalaron que la necesidad de mantenerse en contacto con su familia ha hecho que los medios y la tecnología sean parte de sus vidas cotidianas de forma no anticipada. La mayoría ve televisión dominicana, lee la prensa dominicana, escucha las estaciones de radio dominicanas y vota en las elecciones dominicanas.

En estos días las posibilidades de los medios son casi interminables. "Mensajes de texto," "sexting," "Facebook," citas para salir, citas por TV y páginas de turismo sexual, son medios utilizados por los inmigrantes. Las mujeres dominicanas también han penetrado el campo de los diversos medios de comunicación como medio de mantener las relaciones familiares, y como medio de crear nuevas relaciones. ${ }^{137} \mathrm{La}$ antropóloga Dennis Brennan ha observado que para muchas mujeres dominicanas conservar lazos transnacionales con su hombre extranjero es frecuentemente una tarea diaria. ${ }^{138}$ Para algunas "pasar por la oficina de Codetel para ver si ha recibido algún fax es un ritual diario."139

131. Karin Weyland, Dominican Women con un Pie Aqui y otro Alla en DOMINICAN Migration transnational Perspectives [Perpectivas de la Migración Transnacional DOMINICANa] 165 (Univ. Press Fla. 2004).

132. Duany, supra nota 123, en 206.

133. Id.

134. Entrevista con Dina, supra nota 109.

135. Id.

136. Id.

137. DENISE BRENNAN, What's Love Got TO DO WITH IT? TRANSNATIONAL DESIRES AND SEX TOURISM IN THE DOMINICAN RePUbliC [¿QUÉ TIENE QUE VER EL AMOR CON Esto? Deseos TRANSNACIONALES Y TURISMo SEXUAL EN la REPÚBlica DOMINICANA] 21 (Duke Univ. Press 2004).

138. Id.

139. Id. 
Otras mujeres buscan conocer sus futuros novios y esposos por programas de citas de Internet. ${ }^{140}$ Mantienen relaciones de larga distancia mediante correos electrónicos, mensajes de texto y envío de contenidos eróticos o "sexting."141 Por ejemplo, Veras conoció su esposo cuando una amiga "texteó" su número teléfono a un canal de videos musicales para hacer nuevos amigos. ${ }^{142} \mathrm{Su}$ amiga estaba ya hablando con un hombre cuando recibió otra llamada telefónica, y la amiga le dijo a Veras que contestara el teléfono. ${ }^{143}$ Veras contestó y habló con el hombre por más de una hora. ${ }^{144}$ Ambos se gustaron y comenzaron a hablar por teléfono y a "textearse" más de cinco veces al día hasta que finalmente se conocieron. ${ }^{145}$

Debe ser interesante investigar cuán común es el uso de las tecnologías y los medios sociales en las mujeres inmigrantes en Puerto Rico y los Estados Unidos. Una investigación interesante llevada a cabo en Irlanda encontró que los medios sociales permiten tener un fondo de alerta de información de amigos y conocidos que favorece la vinculación del capital $y$ las comunidades transnacionales de forma no vista previamente. ${ }^{146}$

\section{FORMANDO Y RE-FORMANDO IDENTIDADES}

Las mujeres dominicanas inmigrantes viajan lentamente a través de fronteras físicas $\mathrm{y}$ discursivas. Las identidades son construidas, deconstruidas y reconstruidas en un continuo discursivo y experiencial. La experiencia migratoria sirve como un buque para cruzar fronteras que les permiten nuevas experiencias de transgresión y apoderamiento. Por ejemplo, en el caso de mujeres inmigrantes indocumentadas, la etiqueta "indocumentada" es una identidad impuesta en ellas desde afuera. ${ }^{147}$ Sus

140. Steven Gregory, The Devil Behind the Mirror: Globalization and Politics IN THE DOMINICAN REPUBlic [El DEMONIO DETRÁs DEL ESPEjo: GloballZaCión y POLÍtica EN La REPÚBliCA DOMINICANA] 136-141 (Univ. of Cali. Press 2007).

141. Entrevista con Veras (fecha desconocida) ('Veras' no es el verdadero nombre de la persona entrevistada. Su nombre ha sido cambiado a Veras para proteger su identidad) [en lo sucesivo Entrevista con Veras].

142. Id.

143. Id.

144. Id.

145. Id.

146. Lee Komito, Social Media and Migration: Virtual Community 2.0 [Medios Sociales y Migración: Comunidad Virtual 2.0], 62 J. AM. SOC'Y INFO. SCI. TECH. 1075, 1075 (2011).

147. Silvia Pilar Castro Borrego \& Maria Isabel Romero Ruiz, Repossessing Our Bodies and Ourselves [Reposeyendo Nuestros Cuerpos y a Nosotros Mismos], en CULTURAL MIGRATIONS AND Gendered Subjects: Colonial and Postcolonial Representations of the Female Body 
cuerpos son el crimen: su mera presencia es una transgresión. ${ }^{148}$ Como han señalado Castro y Romero: "[l] os cuerpos de las mujeres siempre han sido, y siguen siendo hoy día el lugar de pecado y transgresión, discriminación y control, explotación y comercialización." 149

Para todas las mujeres entrevistadas el viaje de la República Dominicana a Puerto Rico es el inicio de la "otra" que son, la otra mujer que comenzaron a ser. ${ }^{150}$ Como Gregoria puntualiza:

Me siento preparada, ... Que antes yo era una mujer sumisa, yo era una mujer que duré tres años con el papá de mis hijos. Que si ese plato estaba mal ahí, pues se quitaba porque el hombre habló. Ahora no, te molesta? pues quítalo, mira tengo las pantalones sucios: lávalos. Hoy? No, yo me siento cansada. Yo voy a lavar cuando yo quiera. Si los quieres limpios, pues lávalos. Yo no hacía eso antes. ${ }^{151}$

Las mujeres inmigrantes que vienen a Puerto Rico tienen que afrontar cara a cara la construcción discursiva de las dominicanas en la sociedad Puertorriqueña. ${ }^{152}$ Frecuentemente encuentran un ambiente de xenofobia enraizado en racismo y nacionalismo. ${ }^{153}$ La experiencia de discriminación está asociada con los bajos niveles de autoestima de las mujeres inmigrantes dominicanas. ${ }^{154}$ En Puerto Rico, la construcción acostumbrada del discurso público hacia las mujeres inmigrantes es similar a la de otros países que son marcados por la discriminación. ${ }^{155}$ Maneri, cuando habla sobre la racialización de los inmigrantes en Italia, ha indicado que la representación discursiva de los inmigrantes frecuentemente "moldea las

[Migraciones Culturales $\mathrm{Y}$ Temas de GÉnero: Representaciones Coloniales $\mathrm{Y}$ PostColoniales del CuerPo FEMENINO] 1, 9 (Cambridge Scholars 2011).

148. Id.

149. Id.

150. Entrevista con Grullart, supra nota 43.

151. Id.

152. De Paz Lisk v. Aponte Roque, 124 P.R. Dec. 472 (El Tribunal Supremo consideró inconstitucional la práctica discriminatoria que tuvo lugar en Puerto Rico contra una mujer dominicana a la que no se le habia permitido ser profesora en un colegio público porque era residente legal permanente y no ciudadana de los Estados Unidos).

153. Id.

154. Beverly Araújo Dawson \& Sandra Panshanadeswaran, How Discrimination and Stress Affects Self-Esteem Among Dominican Immigrant Women: An Exploratory Study [Cómo la Discriminación y el Estrés Afecta a la Auto Estima de las Mujeres Inmigrantes Dominicanas: un Estudio Exploratorio], 26 SOC. WORK PUB. HEALTH 60, 60 (2010).

155. Ver infra nota 170. 
formas gramaticales que designan sus actos."156 Son frecuentemente sujetos activos de acciones negativas y problemáticas (ellos desembarcan, prostituyen, participan en trafico de drogas, son recipientes pasivos del gobierno y de las acciones de base comunitaria). ${ }^{157}$

El término racialización puede parecer una representación incorrecta de la relación entre los puertorriqueños y los dominicanos porque es una homogeneización de la construcción de identidades basada en ciudadanía, no en etnia. ${ }^{158}$ No obstante, yo coincido con Duany que en principio cualquier grupo puede ser racializado. Esto es decir, que su identidad es construida desde afuera mediante la asignación de características intelectuales, emocionales, y de comportamiento basadas en el grupo al que pertenecen. ${ }^{159}$ Los dominicanos en Puerto Rico son frecuentemente discriminados porque son percibidos como negros, sucios, vulgares $y$ criminales que están invadiendo el país. ${ }^{160}$ Los chistes populares, en la radio, la televisión, y otros medios, frecuentemente ridiculizan el acento dominicano y sus costumbres. ${ }^{161}$ Brennan, además, señala que el Internet juega un papel importante en la "producción y diseminación de estereotipos basados en raza y destrezas sexuales" sobre las mujeres dominicanas. ${ }^{162}$ Las mujeres dominicanas son asociadas con disponibilidad sexual y son ampliamente anunciadas en Internet. ${ }^{163}$ Gracia recuerda el primer encuentro con un hombre puertorriqueño después de llegar en la yola:

Ese día cuando llegamos nos sacaron del sitio donde estábamos y nos pusieron en otro, y había un Señor que quería quedarse conmigo, que quería que yo me quedara con él tu sabes, que él decía yo te hago los papeles quédate conmigo, que las

156. Marcello Maneri, Media Discourses on Immigration Control Practices and the Language We Live [Discursos Mediáticos Sobre las Prácticas de Control de la Inmigración y el Lenguaje que Vivimos], en Racial Criminalization of Migrants in the 21St Century [Penallzación Racial DE LOS MigRANTES EN EL SIGLO] 77, 78 (Ashgate 2011).

157. Id.

158. Ver infra notas 170-76.

159. Suzanna Oboler, Racializing Latinos in the United States: Toward a New Research Paradigm [Clasificando Latinos en los Estados Unidos: Hacia un Nuevo Paradigma de la Investigación], en IDENTITIES ON THE MOVE TRANSNATIONAL PROCESSES IN NORTH AMERICA AND THE CARIBBEAN BASIN [IDENTIDADES EN MOVIMIENTO: PROCESOS TRANSNACIONALES EN NORTE AMÉRICA y El CARIBE de Basin] 45, 46 (Univ. of Tex. Press 1999).

160. Duany, supra nota 123 , en 214.

161. Id. en 219.

162. BRENNAN, supra nota 137, en 199.

163. Id. en 198-201. Steven Gregory, supra nota 140 en 33, 62, 136-141 (Univ. of Cali. Press 2007). 
dominicanas son mejores, que lo hacen mejor. $\mathrm{Y}$ yo le decía nooooooo... ${ }^{164}$

Como Romelinda Grullón señala, en Puerto Rico existe además una construcción de la mujer dominicana particular. ${ }^{165}$ Ellas son percibidas como prostitutas inmorales y ladronas. ${ }^{166}$ Son acusadas de llevarse los maridos de las mujeres puertorriqueñas y solo casarse por los papeles. ${ }^{167}$ Grullón bromea que "parece que los hombres son vistos como un recurso que escasea."168 Ella añade, no obstante, que la mujer dominicana es además, percibida como una mujer fuerte y trabajadora. ${ }^{169} \mathrm{La}$ construcción de los dominicanos encuentra eco en las experiencias de muchas de las mujeres entrevistadas. ${ }^{170}$

La señora me decía: 'yo no te quiero aquí que mi marido es un hombre enamorado, es medio fresco, tu eres un peligro aquí en mi casa.' Yo estaba loca por ver al marido ese, ja y cuando llegó yo le dije: 'Mire doña, ese hombre está para usted, esa porquería, usted me dice que tenga cuidado con eso. Yo aquí no vine a buscar marido ajeno.' Después nos vimos solas, porque el fulano se le fue y ella me ayudaba a mí y yo la ayudaba a ella. Y así pasamos todo ese tiempo y todavía, esa señora me busca. ${ }^{171}$

Todas las mujeres entrevistadas coinciden, que a pesar de todas las etiquetas sociales y legales impuestas por la sociedad anfitriona, su identidad nacional como dominicanas se mantiene fija. ${ }^{172}$ Para todas ellas, sobrevivir el abuso es una de las características que definen a la mujer que son ahora. ${ }^{173}$ Les ha dado un sentido de apoderamiento. ${ }^{174}$

164. Entrevista con Gracia, supra nota 83.

165. Entrevista con Romelinda Grullón, Dir., Centro de la Mujer Dominicana, (Dec. 17, 2010) [en lo sucesivo Entrevista con Grullón].

166. Id.

167. Id.

168. Id.

169. Id.

170. Entrevista con Grullón, supra nota 165.

171. Entrevista con Gracia, supra nota 83.

172. Ver Jose Itzigsohn \& Carlos Dore Cabral, Competing Identities? Race, Ethnicity and Panethnicity Among Dominicans in the United States [¿Identidades Competitivas? Raza, Etnia y Panétnica Entre Dominicanos en los Estados Unidos], 15 SOC. FOR. 225, 225 (2000).

173. Id. en 241.

174. Id. 
La experiencia migratoria les ha dado además un sentido de estar en control de sus vidas; de independencia y de conocimiento. ${ }^{175}$ Gracia frecuentemente recuerda que cuando vivía en su casa en la República Dominicana, era la única hija y la favorita de su mamá. ${ }^{176}$ Ahora se ve a sí misma como una mujer diferente. ${ }^{177}$ Gracia bromea:

Mira cuando yo llegué yo no sabía nada de nada, porque en mi casa mi mamá no me dejaba hacer nada. Yo no sabía freír ni un huevo, ni hacer arroz. Cuando me casé mi esposo no podía creer que yo no sabía cocinar, decía que no podía creer que se hubiera casado con la única mujer dominicana que no cocinaba. Ahora, ahora yo puedo hacer lo que sea, arreglar, un recorte, soldar un hierro, arreglar gomas, si es echar cemento, pues también, lo que sea, si es cortar patios también lo hago. Estoy ready. ${ }^{178}$

Gregoria es quizás la más visible de las inmigrantes sobrevivientes de violencia doméstica en Puerto Rico. ${ }^{179}$ Ha hecho de esto su negocio, y el negocio de todos; que su historia se cuente. ${ }^{180}$ En la entrevista miró atrás y dijo:

Yo ya no me dejo joder la vida. El trabajo dónde yo entro lo primero que yo digo en la entrevista: Cómo estás? Bien gracias y me dicen esto es así, y asi y yo digo OK Ahora me toca a mí. $\mathrm{Si}$ algún día yo falto, no me hable entre la gente que no somos muchachos y dependiendo como usted me trate, pues bregamos. No acepto que nadie me humille.

\section{Ella continuó:}

Soy una mujer feliz y alegre, divertida. Dónde yo me meto, el que no quepa que se salga que llegue yo. Sabes única, me siento, única. No me he deprimido por eso. Me siento preparada para seguir la vida. Me siento una mujer luchadora, qué te digo, dramática en todo. ${ }^{181}$

175. Entrevista con Gracia, supra nota 83.

176. Id.

177. Id.

178. Entrevista con Grullart, supra nota 43.

179. Firuzeh Shokoo Valle, Del Dolor Sacó la Fuerza, Primera HoRA, (16 de Octubre del 2007).

180. Entrevista con Grullart, supra nota 43.

181. Id. 
Hay también mucha nostalgia por la familia que dejó atrás. ${ }^{182}$ Esta nostalgia se junta con el deseo de "hacer algo que me permita traer a mi familia a Puerto Rico." 183 Cuando Gracia se fue, sus hijos eran tan pequeños que no la recordaban. ${ }^{184} \mathrm{Y}$ cuando finalmente tuvo la posibilidad de regresar a la República Dominicana, después de diez años, sus hijos fueron a recogerla al aeropuerto pero no la reconocieron. ${ }^{185}$ Al principio ella tampoco los reconoció. ${ }^{186}$ Piensa en abrir un negocio que le permita traerlos a Puerto Rico, quizás un restaurante ya que ahora sabe cocinar muy bien. ${ }^{187}$

Las identidades complejas que estas mujeres describen encuentran poca o ninguna representación en el discurso público relacionado con las mujeres dominicanas en Puerto Rico.

\section{SOBRE LA VISIBILIDAD}

En general, las mujeres entrevistadas se sienten invisibles, al no ser tomadas en cuenta y rechazan los pocos espacios de representación. En Puerto Rico las mujeres dominicanas existen en la periferia. Es poco probable que en Puerto Rico alguien pueda llevar a cabo su vida sin interactuar con una mujer dominicana. Las mujeres dominicanas son las principales niñeras de los puertorriqueños y además se han desplazado silenciosamente a otras profesiones. ${ }^{188}$ Pero ellas están dramáticamente pocorepresentadas en los medios. ${ }^{189}$ Los medios sirven como una maquinaria cultural global. ${ }^{190}$ Son los responsables de diseminar el discurso sobre lo que la mujer es, y qué debería ser. ${ }^{191}$ El silencio es una forma de invisibilidad, en esas historias no han incluido a la mujer como sujeto político visible públicamente. ${ }^{192}$

Los medios de comunicación juegan un papel determinante en la creación de la opinión pública. El profesor Nash ha declarado que en el contexto de mujeres migrantes:

\footnotetext{
182. Entrevista con Gracia, supra nota 83.

183. Id.

184. Id.

185. Id.

186. Id.

187. Entrevista con Gracia, supra nota 83.

188. Hernandez Angueira, supra nota 8, en 96; Weyland, supra nota 131, en 165.

189. Id.

190. Ver infra nota 211.

191. Id.

192. Id.
} 
Las noticias, la información, las opiniones expresadas en la representación cultural proyectada, tanto en lo audiovisual como en los medios escritos, constituyen el universo de discursos que crea valores, actitudes $\mathrm{y}$ juicios respecto a nuestro ambiente social, cultural y político. ${ }^{19}$

Nash analiza la representación de las mujeres inmigrantes en los medios españoles y concluye que el compromiso de los medios con el asunto de la inmigración femenina no refleja el peso real en la sociedad. ${ }^{194}$ Afirma que "el discurso de los medios genera un campo de significados fundamentado en el silencio que hace a los inmigrantes invisibles, creando una corriente de opinión pública que minimiza su presencia y reconocimiento." 195

Las mujeres dominicanas, en relación a los medios en Puerto Rico, son en gran parte ausentes en el discurso de los medios y se ven como un producto comercial. Los medios de comunicación, sea la televisión, revistas, internet, $u$ otras formas, proveen modelos con los que las mujeres se comparan. ${ }^{196}$

Esto no quiere decir que no exista ninguna representación de las mujeres dominicanas en los medios. Lo que significa es que no ha existido representación aparte de las mujeres como atletas y en el ámbito del entretenimiento. ${ }^{197}$ La representación más notable fue la representación de una luchadora e inculta trabajadora doméstica llamada Altagracia en una comedia de televisión que se presentó en el $1980 .{ }^{198}$

Esta ausencia de visibilidad es también observada en la prensa escrita. Aunque se ha visto el desafío de su relevancia por su inmediatez con la red

193. Mary Nash, Rethinking Media Representations of Immigrant Women [Replanteándose las Representaciones Mediáticas de Mujeres Inmigrantes], en QUADERNS DE LA MEDITERRANIA 57 (2006).

194. Id. en 58 .

195. Id.

196. INDIA BROWN, MEDIA REPRESENTATION OF YOUNG WOMEN IN THE DOMINICAN REPUBLIC [REPRESENTACIÓN MEDIÁTICA DE MUJERES JóVENES EN LA REPÚbliCA DOMINiCANA] 13 (2010).

197. Id.

198. Eva-María Suárez Büdenbender, Comparing Dominican Linguistic (In) security in the Dominican Republic and in the Diaspora [Comparando lingüistica Dominicana la (in)seguridad en la República Dominicana y en la Diáspora], en Claudia Borgonovo et al eds. SELECTED ProceEdings of THE 12TH HisPaNIC LINGUISTICS SYMPOSIUM, 148-159 (2010) (quoting AIDA DE LA Rosa LA IDENTIDAD CULTURAL DE LA MUJER DOMINICANA DE CLASE TRABAJADORA EN PUERTO RICO: SU ARTICULACIÓN EN LA COMEDIA TELEVISIVA (University of Puerto Rico MA Thesis (2002)). 
social, ésta continúa siendo una importante fuerza discursiva. ${ }^{199}$ La prensa se continúa percibiendo como el recurso de información más serio, confiable e imparcial. ${ }^{200}$ El involucramiento en ciertas cuestiones sociales y políticas ha sido vinculado positivamente con la importancia percibida de la cobertura de las historias en el periódico. ${ }^{201}$ Puerto Rico tiene cuatro periódicos diarios. ${ }^{202}$ Todos ellos tienen alguna forma de versión en línea. ${ }^{203}$ Uno de los periódicos, Primera Hora, incluye información rutinaria a cerca de la República Dominicana, hasta ha designado una página con ese propósito. ${ }^{204}$ Se espera que la cobertura de cuestiones importantes a las mujeres dominicanas migrantes sea el artículo principal. ${ }^{205}$ Sin embargo, la verdad ha sido lo opuesto. Durante los últimos dos años, no ha habido contenido relacionado con las mujeres dominicanas inmigrantes, a pesar de la publicación de más de 2000 historias relacionadas con la política, el deporte y el entretenimiento dominicano. ${ }^{206}$

Solo se han publicado un puñado de historias de mujeres dominicanas en su contexto, no relacionadas con la política, el deporte, la música o el espectáculo. ${ }^{207}$ Las otras historias publicadas sobre las mujeres dominicanas involucran a Sobeida Félix, la mujer fatal del negocio de drogas entre Puerto Rico y República Dominicana y sobre la antigua señorita de la República Dominicana, Amelia Vega, quien recientemente se hizo representante de la Compañía de Turismo de Puerto Rico. ${ }^{208}$ Otras historias tratan de mujeres inmigrantes arrestadas por prostitución y abandono de niños, y mujeres inmigrantes que han sido víctimas de crímenes. ${ }^{209}$

199. Blake D. Morant, Democracy, Choice, and the Importance of Voice in Contemporary Media [Democracia, Elección, y la Importancia de la Voz en los Medios de Comunicación Contemporáneos], 53 DEPAUL L. REV. 943, 965 (2003-2004).

200. Id.

201. Bruce E. Pinkleton \& Erica Weintraub, Individual Motivations, Perceived Media Importance, and Political Disaffection [Motivaciones individuales, la Importancia de la Percepción de los Medios de Comunicación, y la Desafección Politica], 18 POL. COMM. 321, 330 (2001).

202. Id. en 321 .

203. Id.

204. Id.

205. Id.

206. Centro de la Mujer Dominicana, supra nota 14.

207. Id.

208. Id.

209. Id. 
Las únicas noticias generadas por mujeres dominicanas son producidas por el Centro de la Mujer Dominicana. ${ }^{210}$ El Centro ha producido historias relacionadas a cómo las mujeres superan la violencia, los servicios ofrecidos por el Centro, las actividades que las mujeres organizan y las solicitudes para cambios en las políticas públicas. ${ }^{211}$

La organización de las mujeres inmigrantes, a través del Centro, ha llenado un espacio sin oposición y ganado visibilidad. ${ }^{212}$ El Centro, como única organización creada y manejada por mujeres inmigrantes, ha generado un sentido de apropiación y confianza entre las mujeres que les ha permitido participar plenamente. ${ }^{213}$ Están capturando un espacio discursivo vacío y llenándolo con su propio discurso. ${ }^{214}$ El discurso en contra de la violencia doméstica, y la disponibilidad de remedios legales bajo la Ley en Contra de la Violencia de la Mujer, ha permitido a las mujeres dominicanas aglutinarse y reclamar ese espacio. ${ }^{215}$ No debe sorprender que la disponibilidad de protección legal para las sobrevivientes de violencia haya tenido el efecto de revestir a las mujeres inmigrantes en un bloque cohesivo.

Mediante su visibilidad asertiva estas mujeres han logrado no sólo ocupar el espacio físico y discursivo, si no redefinir ese espacio. Haciendo sus voces y cuerpos visibles exponen su humanidad: sus esperanzas, sus opiniones, sus sueños y sus vidas. Su presencia provoca preguntas que demandan respuestas.

\section{CONCLUSIÓN}

El transnacionalismo puede abrir las puertas para el establecimiento de un nuevo cosmopolitanismo y una nueva perspectiva de ciudanía y participación. ${ }^{216}$ Los procesos migratorios han abierto las puertas que permiten a las mujeres migrantes acceso a la esfera pública. Esto es crucial porque históricamente, para que las mujeres puedan mejorar su ciudadanía cultural tienen que entrar a la esfera pública. Las inmigrantes dominicanas

210. Id.

211. Centro de la Mujer Dominicana, supra nota 14.

212. Id.

213. Id.

214. Ver Shani M. King, Race, Identity, and Professional Responsibility: Why Legal Services Organizations Need African [Raza, Identidad, y Responsabilidad Profesional: Por Qué las Organizaciones de Servicios Legales Necesitan Africanos], 18 CORNELL J.L. \& PUB. POL'Y 1, 3 (2008).

215. Violence Against Women Act [Ley Contra la Violencia de Género], supra nota 18.

216. Alexandra Dobrowlsky \& Evangelina Tatsoglou, Crossing Boundaries and Making Connections [Cruzando Fronteras y Haciendo Conexiones], en WOMEN, MIGRATION AND CITIZENSHIP 1 (Ashgate, 2006). 
entrevistadas sienten que la experiencia migratoria les ha permitido tener el control de sus propias vidas de forma que no conciben regresar a casa.

Estas mujeres han superado experiencias terribles de abuso doméstico y han dejado sus países y a sus familias. Además se han organizado a sí mismas y están navegando el espacio hacia la visibilidad y el reconocimiento. El reconocimiento de ellas mismas en sus propios términos por sus propias historias.

Estas historias han comenzado a ser parte de la narrativa de la migración. Tomando prestado de Margaret Montoya, "las narrativas que se enfocan en las experiencias de los extraños apoderan al que cuenta y al que escucha la historia por virtud de su oposición a las formas tradicionales de discurso."217 Como Badiu ha afirmado magistralmente, cuando esas historias son ventiladas "declaran públicamente la existencia y el valor de lo que previamente ha estado sin ser valorado de tal modo que deciden que aquellos quienes han estado aquí pertenecen aquí, y disfrutando de la gente para soltar la expresión 'inmigrantes ilegales'."218

Desde el punto de vista de LatCrit podemos decir que el compartir la narrativa de estas mujeres migrantes nos permite reinterpretar y reposicionar discursos contemporáneos relacionados con la inmigración, de forma que abarquen la perspectiva de "los otros" y apoderen a las comunidades. ${ }^{219}$ Ruitenberg nos recuerda que todos nosotros portamos las marcas de los lugares donde hemos vivido, no importa cuánto tiempo hemos vivido allí. Nos recuerda que, "el ser humano no hace crecer sus raíces de donde depende para su sustento, físico, intelectual o ético. En cambio los nómadas aprendieron que gestos éticos de hospitalidad y apertura a la comunidad que llegue, brindará fortaleza a cualquier lugar donde lleguen." 220

217. Margaret Montoya, Máscaras, Trenzas y Greñas: Unmasking the Self While Embracing Latina Stories and Legal Discourse [Máscaras, Trenzas y Greñas: Enmascarando a Uno Mismo Mientras se Acoge Historias Latinas y el Discurso Legal], en CRITICAL RACE THEORY 522 (Temple Univ. Press 2000).

218. Alain Badiou \& Slavoj Zlzek, Philosophy in the Present [Filosofía en el PRESENTE] 37 (Polity 2009).

219. Berta Esperanza Hernandez-Truyol, Latina Multidimensionality and LatCrit Possibilities: Culture, Gender and Sex [Multidimensionalidad Latina y Posibilidades de LatCrit: Cultura, Género y Sexo], 53 MIAMI L. REV. 811,813 (1999).

220. Claudia Ruitenberg, Deconstructing the Experience of the Local: Toward a Racial Pedagogy of Place [Deconstruyendo la Experiencia de lo Local: Hacia una Pedagogía Racial del Lugar], en PHILOSOPHY OF EDUCATION [FILOSOFÍA DE LA EDUCACIÓN] 212, 219 (2005). 
Como dijo Derrida en Sobre Cosmopolitanismo, "Yo también imagino que estas migraciones han levantado un espacio para la reflexión y para poner a prueba un orden nuevo de ley y democracia.,221

221. Ver generalmente JACQUES DERRIDA, ON COSMOPOLITANISM AND FORGIVENESS [EN COSMOPOLITANISMO Y PERDÓN] (Routledge 2001). 



\title{
REGULACIONES ESTATALES Y LOCALES DE INMIGRACIÓN: LA NECESIDAD PARA UN TRINQUETE BILATERAL
}

\author{
David P Weber *
}

I. INTRODUCCIÓN.

II. El Debate SOBRE El DERECHO DE PRIORIDAD EN INMIGRACIÓN

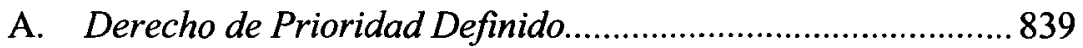

B. El Derecho de Prioridad Relacionado con la Inmigración .... 841

1. Las Raíces Históricas del Derecho de Prioridad .............841

2. Tendencias Modernas sobre el Derecho de Prioridad.....843

a. Regulaciones de Inmigración Estatales .....................843

b. Regulaciones de Inmigración Estatales .....................845

i. Leyes y Ordenanzas Locales .................................845

ii. Leyes y Ordenanzas Locales

Confirmadas

3. Más allá de E-Verify y el Empleo: S.B. 1070 y sus

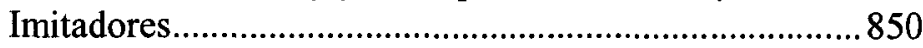

4. 2011: La Corte Suprema Actúa .......................................851

a. Cámara de Comercio de los Estados Unidos v. Whiting...

b. Mártinez v. Regentes de la Universidad de

California. 854

c. Hazleton v. Pensilvania............................................. 857

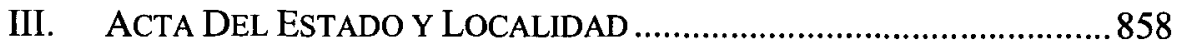

IV. PROPONIENDO UN TRINQUETE BILATERAL ....................................863

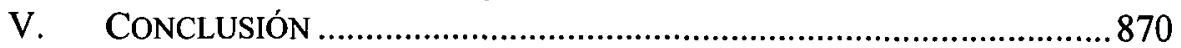

\section{INTRODUCCIÓN}

El problema del principio de prioridad federal en el ámbito de la inmigración se ha discutido cada vez más y más en los círculos académicos, en los tribunales, e incluso en la conciencia pública ${ }^{1}$ desde que el caso de

* Professor Asociado en la Escuela de Leyes de Creighton. El autor quisiera agradecer a LatCrit por la oportunidad de presentar su ensayo en el Intercambio Anual Sur-Norte 2011 en Teoria, Cultura y Leyes (SNX) y a sus compañeros panelistas por todos los comentarios recibidos sobre el tema, y la investigadora Emma Nagengast por su ayuda editando, comentando y asistencia investigando. Profesor Asociado en la Escuela de Leyes Creighton.

1. Ver, por ejemplo, Rich Samp, Note to the DOJ: The Supreme Court is Watching You on Preemption [Nota al Departamento de Justicia: La Corte Suprema Esta Prestando Atención al Principio de Prioridad], ForBes, 27 de Mayo del 2011, http://blogs.forbes.com/docket/ 
De Canas v. Bica fue decidido en $1976 .^{2}$ A medida que leyes más estrictas de control de la inmigración fueron promulgadas en los 1980 y 1990, el debate sobre el derecho de prioridad se ha intensificado. ${ }^{3}$ Desde entonces, las numerosas leyes promulgadas tanto en jurisdicciones estatales como en locales han producido el surgimiento de literatura sobre el tema de la prioridad federal (explícito o implícito), ${ }^{4}$ la regulación local de la inmigración, ${ }^{5} \mathrm{y}$ el trasfondo potencialmente discriminatorio que parece impregnar este debate. ${ }^{6}$

Arizona, aunque recibe la mayor cobertura, no es el único Estado que opta por regular la inmigración, ya sea directa o indirectamente. ${ }^{7}$ A la luz

2011/05/27/note-to-doj-the-supreme-court-is-watching-you-on-preemption.html (última visita el 8 de Febrero del 2012).

2. De Canas v. Bica, 424 U.S. 351(1976).

3. Ver generalmente Immigration Reform and Control Act (IRCA) of 1986 [Ley de Reforma y Control de Inmigración], Pub. L. No. 99-603, 1986 U.S.C.C.A.N. (100 Stat.) 3359 (codificado de acuerdo a las modificaciones en secciones del Titulo 8 del U.S.C.); Immigration Act of 1990 [Ley de Inmigración del 1990], Pub. L. No. 101-649, 1990 U.S.C.C.A.N. (104 Stat.) 4978 (codificado de acuerdo a las modificaciones en secciones del Titulo 8 del U.S.C.).

4. Ver, por ejemplo, Clare Huntington, The Constitutional Dimension of Immigration Federalism [La Dimensión Constitucional del Federalismo en Inmigración], 61 VAND. L. REV. 787 (Abril 2008); Federal Statutes and Regulations, Federal Preemption of State Law [Estatutos Federales y Reglamentos, El Principio de Prioridad], 114 HARV. L. REV. 339 (Nov. 2000); Gary Endelman \& Cynthia Juarez Lange, State Immigration Legislation and the Preemption Doctrine [Legislación de Inmigración del Estado y la Doctrina del Principio de Prioridad], 1968 PLV/Corp. 123 (Octubre. 14-15 2008); Caleb Nelson, Preemption, 86 VA. L. REV. 225 (Marzo 2000).

5. Ver Immigrant Policy Project [Proyecto de Política de Inmigración], National Conference of State Legislatures, disponible en http://www.ncsl.org/default.aspx?tabid=19897 [en lo sucesivo Proyecto de Política de Inmigración] (haciendo resumen de la legislación de inmigración que se aprobó a nivel estatal en 2009, 2010, 2011 y teniendo en cuenta que en el primer trimestre de 2011, los legisladores en todos los cincuenta estados y Puerto Rico habían introducido 1.538 propuestas relacionadas a los inmigrantes y refugiados).

6. Ver, por ejemplo, Lindsay Nash, Expression by Ordinance: Preemption and Proxy in Local Legislation [Expresión a través de una Ordenanza: El Principio de Prioridad y El Poder en Legislación Local], 25 GEO. IMMIGR. L.J. 243, 247-51 (2011).

7. Ver L.B. 48, 102d Leg., 1st Sess. (Neb. 2011) (proponiendo que Nebraska adopte la "Ley de Control de Inmigración Ilegal"); L.B. 569, 102d Leg., 1st Sess., (Neb. 2011) (exigiendo a los empleadores de Nebraska que utilicen el programa federal E-Verify para verificar la autorización del empleado para trabajar en los Estados Unidos); L. Res. 224, 100th Leg., 2d Sess. (Neb. 2007) (proponiendo que Nebraska adopte el "Acuerdo de Memorandos, conforme a lo autorizado por la sección 287 (g) de la Reforma Federal de Inmigración Ilegal y Responsabilidad del Inmigrante de 1996"); ver también H.B. 7089, 113 th Leg., Reg. Sess. (Fla. 2011); S.B. 104, 151 st Gen. Assemb., Reg. Sess. (Ga. 2011); H.B. 1969, 97th Gen. Assemb., 1st Sess. (IIl. 2011); S.F. 102. 84th Gen. Assemb. (lowa 2011); S.B. 6, 11 th Gen. Assemb., Reg. Sess. (Ky. 2011); H.B. 3341, 76th Leg., Reg. Sess. (Or. 2011); H.B. 1375, 82d Leg, Gen. Sess. (Tex. 2011) (prohibiendo que las autoridades estatales o locales adopten leyes u ordenanzas que restringen la aplicación de las leyes federales de inmigración); Guest Worker Program Act [Ley del Programa de Trabajadores Huéspedes], H.B. 116, 59th Leg., Gen. Sess. 
de tres decisiones de la Corte Suprema en el 2011, la Cámara de Comercio de los Estados Unidos v. Whiting, ${ }^{8}$ Martínez v. Regentes de la Universidad de California ${ }^{9}$ y Hazleton v. Pensilvania ${ }^{10}$ casos que parecen abarcar una interpretación más estrecha sobre el derecho de prioridad, este artículo propone un trinquete bidireccional que permitirá a los estados y localidades la habilidad de crear leyes de inmigración más equilibradas.

En la situación actual, aunque los estados tienen prohibido criminalizar violaciones en el ámbito laboral relacionadas con la inmigración, ${ }^{11}$ a los estados y localidades se les está permitido regular en la materia de inmigración, pero básicamente sólo en una manera que favorezca la aplicación a través del programa $287(\mathrm{~g}){ }^{12}$ y Comunidades Seguras. ${ }^{13}$ También está la tendencia puesta de moda por Arizona e imitada en toda la nación, consistente en la aplicación a través del desgaste, alzando el costo de negocio de aquellos que utilicen la mano de obra de inmigrantes indocumentados (esto último conseguido en gran parte por la amenaza de la revocación de la licencia). ${ }^{14}$

(Utah 2011); Utah Illegal Immigration Enforcement Act [Ley de Ejecución de Inmigración Ilegal de Utah], H.B. 497, 59th Leg., Gen. Sess. (Utah 2011) (formando el Pacto de Utah tratando los temas de empleo, la identificación y verificación).

8. Chamber of Commerce of U.S. v. Whiting, 131 S. Ct. 1968, 1972 (2011) (sosteniendo la ley de Arizona que, entre otras cosas, exige el uso de E-Verify, asi como establece sanciones para quienes empleen inmigrantes ilegales).

9. Martinez v. Regents of the Univ. of Cal., 131 S. Ct. 2961 (2011) (negando avocación en un caso que cuestiona la ley de California que permite obtener matricula estatal a inmigrantes indocumentados que cumplen con ciertos requisitos). El caso es importante con respecto al principio de prioridad (junto a Whiting y Hazleton) porque establece el punto de vista de la Corte de Roberts sobre el principio de prioridad. En Martinez, la pregunta sobre el principio de prioridad posiblemente tuvo gran influencia, ya que 8 USC $§ 1623$ (a) establece expresamente que los estados no pueden proporcionar beneficios de educación superior basándose en la estancia dentro de un estado (o subdivisión política). Ver id.; 8 U.S.C. $\$ 1623(\mathrm{a})$.

10. City of Hazleton v. Lozano, 131 S. Ct. 2958, 2958 (2011) (reenviando para reconsideración una decisión del Tercer Circuito que había revocado una ordenanza local que prohibe la contratación de y el alquiler de un lugar de residencia a los inmigrantes indocumentados basándose en el principio de prioridad).

11. Ver Whiting, $131 \mathrm{~S}$. Ct. at 1973 (afirmando que "la ley federal de inmigración tiene derecho de prioridad sobre cualquier ley estatal o local que imponga sanciones civiles o penales (que no sea a través de licencias y leyes similares), a quienes empleen ... a inmigrantes indocumentados") (citando 8 U.C.S. § 1324a(h)(2)).

12. Ver Immigration and Nationality Act [Ley de Inmigración y Nacionalidad], 8 U.C.S. $\S$ 1357(g) (codificando el programa 287(g)).

13. Ver Secure Communities [Comunidades Seguras], U.S. IMmigRation AND CuSTOMS ENFORCEMENT disponible en $\mathrm{http}: / \mathrm{www}$.ice.gov/secure_communities/ (definiendo comunidades seguras) (última visita el 19 de marzo del 2012).

14. Ver S.B. 1070, 49th Leg., 2d Sess. (Ariz. 2010). 
Este artículo propone un enfoque más amplio en relación a las regulaciones subfederales a favor de la inmigración con el propósito de nivelar el campo legislativo actual. Bajo el actual enfoque sobre el derecho de prioridad, apoyado por muchos que razonablemente temen por una discriminación subfederal incontrolada en contra de los inmigrantes, el Congreso ha otorgado a los estados el derecho de actuar en este campo exclusivamente en una dirección a favor de la aplicación. De acuerdo a este enfoque, independientemente de la dudosa legalidad de leyes como la S.B. 1070 de Arizona, la L.B. 48 de Nebraska, ó la H.B. 87 de Georgia, estas leyes continúan proliferando. ${ }^{15}$ En vez de sólo permitir propuestas a favor de la aplicación, una propuesta más flexible para analizar cómo interpretar el derecho de prioridad debe ser empleada para otorgarle a los estados la habilidad de generar enfoques variados y más adaptables en el asunto de inmigración ilegal. $^{16}$

La primera parte de este artículo, presenta brevemente el debate histórico sobre las regulaciones federales de inmigración, los estándares del derecho de prioridad y posteriormente analiza casos recientes enfocados especialmente en el derecho de prioridad. La segunda parte se enfoca en varias regulaciones subfederales promulgadas o propuestas en Nebraska en el 2010 y 2011 que reflejan el gran debate que se libra a nivel nacional. Finalmente, a la luz de tres decisiones recientes de la Corte Suprema, la tercera parte propone un análisis más progresista basado en las regulaciones estatales y locales de inmigración para otorgarle a los estados la habilidad de crear e implementar legislaciones positivas de inmigración. ${ }^{17}$ La tercera parte sugiere también un enfoque más flexible basado parcialmente en el acceso de inmigrantes a ciertos derechos esenciales, incluyendo empleo,

15. Ariz. S.B. 1070; Ga. S.B. 104; Neb. L.B. 48.

16. Por ejemplo, la ley recientemente aprobada de Utah que permitiria al estado, en conjunto con el gobierno de México, otorgar permisos de trabajo a los inmigrantes indocumentados en el estado requiere aprobación previa del gobierno federal, pero esta pre-aprobación no parece estar próxima. Ver Utah Illegal Immigration Enforcement Act [Ley de Ejecución de Inmigración Ilegal], Utah H.B. 497; Stephen Dinan, Holder Eyes Utah Guest-Worker Law, ThE WASHINGTON TimES, 2 de Mayo del 2011, disponible en http:/www.washingtontimes.com/news/2011/may/3/holder-eyes-utah-guest-workerlaw/?page=all\#pagebreak.

17. Por el momento, la decisión de Whiting está parcialmente en desacuerdo con las decisiones en casos como la decisión del Tercer Circuito en Hazleton y la decisión del Tribunal del Distrito de los Estados Unidos para el Distrito del Norte de Texas en Villas at Parkside Partners $v$. Farmer's Branch. Ver Hazleton, Pa., $131 \mathrm{~S}$. Ct. at 2958; Villas at Parkside Partners v. Farmer's Branch, Tex., 701 F. Supp. 2d 835,835 (N.D. Tex. 2010). Ya que el título 8 U.S.C. $\$ 1324 a(h)(2)$ contiene una cláusula restrictiva que sólo beneficia el aumento de reglamentos de ejecución, el régimen legal actual está, por su propia naturaleza, a favor de la ejecución. Un enfoque más flexible con respecto a la regulación estatal al menos podría permitir la incorporación de otras propuestas. Ver 8 U.S.C. $§ 1324 \mathrm{a}(\mathrm{h})(2)(2006)$. 
vivienda, transporte, educación y servicios públicos. El artículo concluye que un enfoque con una visión más progresista y pro-inmigrante podría igualar el ámbito jurídico y así servir mejor a los intereses federales y a los intereses de los estados en regular la inmigración.

\section{El Debate Sobre el Derecho de Prioridad en INMIGRaCión}

En mayo del 2011, el Tribunal Supremo anunció su decisión en la Cámara de Comercio de los Estados Unidos v. Whiting ${ }^{18}$ confirmando la ley de Arizona del 2007 que permite a las correspondientes autoridades del estado suspender o revocar ciertas licencias de empleo a aquellos negocios que "conscientemente $o$ intencionalmente contraten" a inmigrantes indocumentados. ${ }^{19}$ La principal razón para defender la ley fue el lenguaje explícito del estatuto que permite a los estados imponer sanciones "por medio de licencias y leyes similares" (en adelante la "Cláusula Restrictiva"), lo cual ha sido regularmente considerado como una esfera de regulación estatal. ${ }^{20}$ Whiting debe también ser considerado en el contexto del análisis del derecho de prioridad en inmigración de los últimos treinta años, el cual parece estar alejándose de un marco amplio del derecho de prioridad (aunque no sea de manera uniforme). ${ }^{21}$ En cambio, todos los análisis del derecho de prioridad en materia de inmigración necesitan ser observados a la luz de tendencias históricas sobre al análisis del derecho de prioridad en general.

\section{A. Derecho de Prioridad Definido}

Antes de centrarse en la aplicación de la doctrina específica de inmigración, es necesario un breve resumen sobre el derecho de prioridad federal para entender sus fundamentos constitucionales. Bajo la ley federal, generalmente hay dos tipos de derecho de prioridad: explicito e implícito, y ambos tipos surgen o de la Cláusula de Supremacía de la Constitución Estadounidense $o$ del lenguaje de un estatuto federal declarando la

18. Whiting, $131 \mathrm{~S}$. Ct. en 1973.

19. ARIZ. REV. STAT. ANN. $\S \S 23-211,212,212.01$ (2010) (West). Esta ley no debe ser confundida con la famosa S.B. 1070, que es mucho más amplia en su alcance y propósito, y que ha sido, parcialmente invalidada en base al derecho de prioridad por la Corte Suprema de E.E.U.U. en el 2012. Ver Arizona v. U.S., 132 U.S. 2492, 2497-98, 2510 (2012) (invalidando las provisiones que crean nuevos delitos estatales para violaciones de inmigración basándose en el derecho de prioridad). Ver también Ariz. S.B. 1070.

20. 8 U.S.C. $\S 1324 \mathrm{a}(\mathrm{h})(2)$.

21. Ver infra notas $32-40 \mathrm{y}$ el texto adjunto. 
prevalencia de la ley federal. ${ }^{22}$ Sencillamente, la Cláusula de Supremacía prohíbe la existencia de cualquier ley subfederal que "interfiera con o sea contraria a" la ley federal, ${ }^{23}$ mientras que el derecho de prioridad explícito surge cuando el Congreso, a través de un estatuto, mantiene explícitamente que cualquier ley subfederal sea desplazada. ${ }^{24}$

En contraste con el derecho de prioridad explícito, el derecho de prioridad implícito puede ocurrir de dos maneras: "principio de prioridad en la materia" o "principio de prioridad en caso de conflicto." Una manera en la que encontramos el "principio de prioridad en la materia" es cuando la ley federal es tan extensa en un área que se considera que regula esa materia en exclusividad. ${ }^{25}$ Son esas situaciones en las que la legislación federal es "tan dominante que es razonable inferir que el Congreso no dejó espacio para que los estados puedan complementarla," las leyes subfederales serán derogadas aunque no estén en conflicto directo con el derecho federal en cuestión. ${ }^{26}$ Otras técnicas que los tribunales han usado para detectar el "principio de prioridad en la materia" son: el hecho de si "el interés federal en ese ámbito es suficientemente dominante" ${ }^{\text {"27 }}$ y si "el objetivo que se intenta obtener mediante la legislación federal y el carácter de las obligaciones impuestas por ésta revelan el mismo propósito." 28

El otro tipo de derecho de prioridad implícito es el "derecho de prioridad en caso de conflicto." ${ }^{29}$ Éste existe donde una ley subfederal "permanece como un obstáculo para la consecución y ejecución de los propósitos y objetivos del Congreso," o no es posible para una parte cumplir con el derecho federal y el subfederal al mismo tiempo. ${ }^{30}$ Un ejemplo relacionado a la inmigración sería el de las localidades que

22. U.S. CONST. art. VI cl. 2; ver también English v. General Elec. Co, 496 U.S. 72, 78 (1990) ((afirmando que "el Congreso puede definir explícitamente la medida en que sus leyes tienen derecho de prioridad sobre las leyes estatales") (citando Shaw v. Delta Air Lines, Inc. 463 U.S. 85, 95-98(1983))).

23. U.S. CONST. art. VI cl. 2; English, 496 U.S. en 78.

24. English, 496 U.S. 72.

25. Rice v. Santa Fe Elevator Corp., 331 U.S. 218, 230 (1947).

26. Rice, 331 U.S. en 230.

27. Schneidewind v. ANR Pipeline Co., 485 U.S. 293, 300 (1988).

28. Rice, 331 U.S. en 230.

29. Ver, por ejemplo, Hines v. Davidowitz, 312 U.S. 52 (1941) (demostrando que uno de los primeros casos de conflicto de derecho de prioridad está relacionado con el tema de inmigración). En 1939, Pennsylvania intentó adoptar una ley de registro de extranjeros requiriendo una tarjeta de identificación, pero la Corte Suprema dictamino que la Ley de Registro Federal de Extranjeros, que no contiene ninguna exigencia de una tarjeta de identificación, tiene derecho de prioridad y sustituye la ley estatal. Ver id en 74.

30. Hines, 312 U.S. en 67. 
promulgan ordenanzas de tipo santuario para impedir o desbaratar la expulsión de inmigrantes no autorizados. ${ }^{31}$

\section{B. El Derecho de Prioridad Relacionado con la Inmigración}

\section{Las Raíces Históricas del Derecho de Prioridad}

Una conclusión razonable después de revisar el análisis del derecho de prioridad relacionado con la inmigración es que se parece fuertemente al camino de un río en que ha cambiado drásticamente y a menudo de manera imprevisible a lo largo del tiempo. Antes de discutir el derecho de prioridad, sin embargo, es necesario primero llegar a la autoridad subyacente para legislar. Con respeto a eso último, durante la mayor parte de los primeros cien años de los Estados Unidos, la ley de inmigración fue mayormente ignorada a nivel federal. ${ }^{32}$ No fue hasta el final de los años 1800 y los casos fundamentales de Chae Chan Ping v. United States (el caso de La Exclusión de los Chinos), Fong Yue Ting v. United States, Ekiu v. United States, Henderson v. Mayor of City of New York, y Chy Lung v. Freeman que el tribunal reconoció, con bastante fuerza, el papel plenario y exclusivo del Congreso en materia de inmigración. Siguiendo las decisiones de esos casos y la necesidad que algunos comentadores han identificado de tener leyes de inmigración uniformes y su aplicación, ${ }^{33}$ empieza a ser aparente que un desafío sobre el derecho de prioridad podría surgir cada vez que un estado o localidad aprueba una ley relacionada en alguna manera con la inmigración.

La posibilidad de desafío al derecho de prioridad está presente cada vez que aparece una regulación subfederal, pero eso no implica que estos estatutos y ordenanzas deban ser reemplazados y no ejecutables. ${ }^{34}$ De hecho, han surgido algunas distinciones principales. En el caso De Canas $v$. Bica, considerado seminal del análisis moderno sobre el derecho de prioridad en el derecho de inmigración, el Tribunal decidió que "el hecho

31. Ver generalmente United States v. Merkt, 794 F.2d 950 (5th Cir. 1986).

32. Ver generalmente John Higham, American Immigration Policy in Historical Perspective [Ley Americana de Política de Inmigración en Perspectiva Histórica], 21 LAW \& CONTEMP. ProBS. 213 (1956).

33. Ver Huyen Pham, The Inherent Flaws in the Authority Position: Why Inviting Local Enforcment of Immigration Laws Violates te Constitution [Los Defectos Inherentes en la Posición de Autoridad Inherente: ¿Por qué Permitir la Ejecución de Leyes de Inmigración Localmente Viola la Constitución], 31 FLA. ST. U. L. REV. 965, 1003 (2004).

34. Ver, por ejemplo, Whiting, $131 \mathrm{~S}$. Ct. at 1970 (concluyendo que la ley federal no tiene derecho de prioridad sobre la "Ley de Trabajadores Legales de Arizona"); ver también ARIz. REV. STAT. ANN. §§ 23-211, 212, 212.01 (2010) (West); De Canas, 424 U.S. 351 (1976). 
de que los extranjeros sean objeto de un estatuto no convierte a éste en una regulación de la inmigración, que es esencialmente una determinación de quién debe o no debe ser admitido para entrar al país, y las condiciones bajo las cuales un candidato legal puede quedarse." $35 \mathrm{El}$ Tribunal señaló que "no parece existir un... interés federal en una situación en la que la ley estatal está creada para remediar los problemas locales, y se aplica sólo a empresarios locales, y sólo con respecto a esos individuos" que el gobierno federal ha considerado no autorizados para trabajar en el país. ${ }^{36}$

A pesar de que el caso de De Canas fue anulado en términos legales con la promulgación de la Ley de Reforma de Inmigración y Control (IRCA) ${ }^{37}$ la decisión fundamental del caso de que las leyes que afectan a los inmigrantes no son necesariamente leyes que regulan la inmigración no fue abandonada. ${ }^{38}$ De hecho, otros eruditos ya han observado que algunos tribunales modernos tienden a ser reticentes a utilizar teorías implícitas del derecho de prioridad siempre que sea posible. ${ }^{39}$ Tanto legisladores como otros grupos abogadores se han aferrado al análisis del derecho de prioridad y la lectura de hojas de té en un esfuerzo para promulgar u oponerse a las leyes locales. ${ }^{40}$

35. De Canas, 424 U.S. en 355.

36. Id.

37. Immigration Reform and Control Act [Acto de la Reforma de Inmigración y Control], 8 U.S.C. $\S \S 1160,1187,1188,1255(a), 1324 a, 1324 b, 1364,1365$ (1986) (con respecto al empleo de inmigrantes no autorizados).

38. Ver Toll v. Moreno, 458 U.S. 1, 17 (1982) (sosteniendo que una ley de la Universidad del Estado de Arizona que no permitía a las personas categorizadas como "no-inmigrantes" ni a sus dependientes obtener la condición de residentes del estado, viola la Clausula Suprema de la Constitución de E.E.U.U.).

39. Ver, por ejemplo, Cristina M. Rodriguez, The Significance of the Local in Immigration Regulation [El Significado de la Regulación Local de Inmigración], 106 MICH. L. REV. 567 (2008).

40. Ver Respuesta del Apelante en 15-16 Lozano 131 S. Ct. 2958 (2011) (No. 07-3531), 2008 WL 3989656 at ${ }^{*} 15-* 16$ (argumentando de que no hay derecho de adquisición preferente sobre las ordenanzas señalando que "los apelados van a extremos para convertir este permiso expreso en una prohibición expresa, resucitado una teoría descabellada . . la cual propone que el titulo 8 del USC $\S$ 1324a (h) (2) permite a una ciudad imponer sanciones de licencia sólo después de que el empleador haya sido juzgado en un proceso penal federal o civil y se haya determinado que infringe una cláusula de IRCA") (citando informe del apelado en 75); Respuesta del Apelante en 18 U.S. v. Arizona, 641 F.3d 339 (9th Cir. 2011) (No. 10-16645), 2010 WL 5162517 en *18 (citando

Tampoco pueden los Estados Unidos establecer derecho de adquisición preferente sólo por demostrar que existe una diferencia entre la ley estatal y federal. Los Estados Unidos no ha identificado un conflicto real entre la sección 5 (C) y la ley federal, ya que Estados Unidos no ha demostrado que el propósito del Congreso era proteger a los empleados no autorizados de las sanciones del Estado

(citas omitidas)). 


\section{Tendencias Modernas sobre el Derecho de Prioridad}

Dado que el Derecho común de la última década se ha desarrollado a un ritmo vertiginoso, los legisladores han intentado varias estrategias para minimizar el riesgo de una batalla sobre el derecho de prioridad. ${ }^{41} \mathrm{~A}$ la luz de esta ola reciente de litigios subfederales ${ }^{42}$ y acciones de la Corte Suprema de 2011 en estos casos, ${ }^{43}$ el autor entiende que se está a punto de provocar otro cambio en el enfoque sobre el derecho de prioridad relacionado con la inmigración. El aspecto más interesante sobre este cambio está en la extensión potencial que los Estados pueden experimentar en su rol con respecto a la inmigración.

\section{a. Regulaciones de Inmigración Estatales}

En 2007, Arizona pasó una ley requiriendo a los empresarios utilizar E-Verify ${ }^{44}$ para confirmar la autorización de trabajo de cada empleado. Esta ley también contenía sanciones consistentes en la revocación de licencias a los empresarios que no cumplían con las prohibiciones federales en relación a la contratación de inmigrantes no autorizados. Aunque lejos de ser tan radical como la ley de Arizona del 2010 conocida como S.B. 1070, la ley del 2007 ha sido percibida como susceptible de un desafio sobre el derecho de prioridad. ${ }^{45}$ Como se expuso en la Parte I.A.4.a., en el 2011 el Tribunal Supremo rechazó el argumento del derecho de prioridad por algunas razones muy específicas. Aunque de gran interés mediático, la decisión en Whiting no fue inesperada ya que tanto el Tribunal del Distrito como el Noveno Circuito habían ratificado la ley, y aunque los argumentos

41. Ver, por ejemplo, Ley de Ejecución de Inmigración Ilegal de Utah, H.B. 497; ver también supra notas 8, 10-11 y el texto adjunto.

42. Ver supra nota 7.

43. Ver Regents of the Univ. of Cal, 131 S. Ct. en 2961; Whiting, 131 S. Ct. en 1968; Hazleton, Pa., 131 S. Ct. en 2958; Arizona, 132 U.S. 2492.

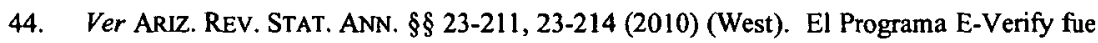
promulgado oficialmente bajo el título "Programa Piloto Básico," y ha sido sometido a tratamiento bastante tumultuoso en su corta historia. Ver Illegal Immigration Reform and Immigrant Responsibility Act of 1996 [Ley de Reforma de Inmigración Ilegal y de Responsabilidad del Inmigrante de 1996], Pub. L. No. 104-208, $\S \S 401-405,110$ Stat. 3009, 3009-655 to 3009-666. La ley establece explícitamente que el Congreso no puede ordenar su uso. 8 U.S.C. $\$ 1324 \mathrm{a}-1324 \mathrm{~b}$ (2006).

45. Ver, por ejemplo, Fawn Johnson, High Court Could Lean Toward States on Immigration [Tribunal Superior Podria Inclinarse a Favor de los Estados Sobre el Tema de Inmigración], NAT'L J. DAILY, 9 de Diciembre del 2010, disponible en 2010 WLNR 24335634; Stephen Dinan, U.S. pushes Everify For Hires Few Agencies Employ System [EE.UU. Presiona el Uso de E-verify Para el Empleo Pocas Agencias Usan el Sistema], WASHINGTON TIMES, 25 de septiembre del 2007, en 1. 
que enfatizaron los Jueces Breyer ${ }^{46}$ y Sotomayor ${ }^{47}$ sobre el delicado balance obtenido por IRCA fueron bastante convincentes, no fueron suficientes para superar lo que el Juez Roberts vio como el lenguaje literal del estatuto.

Curiosamente, la batalla sobre el derecho de prioridad no sólo ha sido considerada por aquéllos a favor de la legislación pro-inmigrantes. La decisión en Martínez, donde California otorgó una matrícula estatal a inmigrantes no autorizados basándose en la localización de la escuela superior donde se habían graduado, parece ir más allá que Whiting ya que podría decirse que permite al estado tomar acción incluso más allá del lenguaje del estatuto. ${ }^{48}$ La legislación de Martínez fue impugnada en base al derecho de prioridad ${ }^{49}$ por violar las prescripciones federales en materia de beneficios a inmigrantes no autorizados basándose en su residencia. ${ }^{50}$

En Martínez, los diferentes argumentos sobre el derecho de prioridad expreso, el principio de prioridad en caso de conflicto y el principio de prioridad en la materia no tuvieron éxito a la hora de persuadir al Tribunal a intervenir en la materia e igualmente, el Tribunal Supremo de California tampoco aceptó que la ley debiera ser reemplazada. ${ }^{51}$ Considerando estos casos juntos, ${ }^{52}$ podría parecer que el Tribunal se está inclinando hacia una interpretación más limitada sobre el derecho de prioridad que el que previamente ha sido aplicado, usando razonamientos similares a los que se usaron en De Canas sobre el hecho de que las regulaciones, aunque afecten a inmigrantes, no se consideraron reguladoras de la inmigración. ${ }^{53} \mathrm{Si}$ consideramos aisladamente a estos dos casos, no parece que el Tribunal esté promoviendo la decisión en De Canas, pero tanto la decisión en Hazleton

46. Whiting, $131 \mathrm{~S}$. Ct. at 1987-97 (Breyer, J., opinión disidente), razonando que, el Congreso no tenía la intención de que su uso del término 'licencia' creara tan amplia exención, porque eso permitiría que los estados destripen la Ley federal del derecho de prioridad, de hecho permitiría subvertir la Ley en sí, al socavar los esfuerzos del Congreso 1) para proteger a los trabajadores legales de la discriminación basada en origen nacional y 2) para proteger a los empleadores legales contra la persecución o castigo erróneo.

47. Id. en 1998-2007 (Sotomayor, J., opinión disidente).

48. Ver Martinez v. The Regents of the Univ. of Cal., 241 P.3d 855, 861 (Cal. 2010).

49. Informe del Demandante en *6-8, *16-*20 Martinez, 241 P.3d en 855 (2010) (No. 101029), 2011 WL 549168.

50. Ver Martinez, 241 P.3d en 860; ver también, 8 U.S.C. $\$ 1623$ (a) (que prohíbe expresamente a los estados de proporcionar cualquier beneficio de educación superior basada en la residencia).

51. Ver Martinez, 241 P.3d en 855, cert. negado, 131 S. Ct. 2961 (2011).

52. Ver Regents of the Univ. of Cal., 131 S. Ct. en 2961; Whiting, 131 S. Ct. en 1970; ver también, De Canas, 424 U.S. en 355.

53. Id. 
como la discusión que se avecina respecto a S.B. 1070 pueden continuar viendo que la Corte está acotando cada vez más el campo de batalla sobre el derecho de prioridad a favor de encontrar un rol subfederal más amplio en el debate.

\section{b. Regulaciones de Inmigración Estatales}

\section{i. Leyes y Ordenanzas Locales}

Como se señaló anteriormente, desde el cambio de siglo los estados y las localidades han comenzado a considerar y a promulgar leyes relacionadas con los inmigrantes y/o la inmigración de una manera cada vez más frecuente $\mathrm{y}$ obteniendo diferentes resultados. ${ }^{54}$ Recientemente, tres lugares principales han probado la validez de este tipo de ordenanzas locales: Texas, ${ }^{55}$ Missouri, ${ }^{56}$ y Pensilvania. ${ }^{57}$ Estas tres jurisdicciones han promulgado estatutos similares que intentaron bien reducir la contratación de inmigrantes ilegales o restringir la capacidad de los inmigrantes no autorizados de alquilar una vivienda, por lo general, apoyándose en las disposiciones anti-refugio, o las dos. ${ }^{58}$

Las ordenanzas fueron impugnadas tanto en base al derecho de prioridad como en base al derecho a un proceso con todas las garantías de la ley, obteniendo diferentes resultados como las ordenanzas de Hazleton, Pensilvania y Farmers Branch, las de Texas fueron derogadas inicialmente, y la ordenanza de Valley Park, Missouri aprobada. ${ }^{59}$

La Ordenanza de Farmers Branch pretendía requerir licencias de residencia, ${ }^{60}$ pero se basó en gran medida en la clasificación de elegibilidad

54. Ver Villas at Parkside Partners, 701 F. Supp. en 835.

55. Ver Gray v. City of Valley Park, Mo., 567 F.3d 976 (8th Cir. 2009).

56. Ver Lozano v. City of Hazleton, 496 F.Supp. 2d 477 (M.D. Penn. 2007).

57. Ver Lozano v. City of Hazleton, 620 F.3d 170, 176 (3rd Cir. 2010) (hablando sobre las ordenanzas de la ciudad de Hazleton: Illegal Immigration Relief Act Ordinance ("IIRAO") [Ordenanza del Acto de Alivio de Inmigración Ilegal], Ordenanza No. 2006-18, modificada por la Ordenanza No. 2006-40, y Ordenanza No. 2007-6 y la Ordenanza de Registración de Renta [Rental Registration Ordinance] ("RO") Ordenanza No. 2006-13); Gray, 567 F.3d en 976 (afirmando que "la ordenanza 1722 prohíbe a todas las entidades de negocio en Valley Park emplear a extranjeros sabiendo que no están autorizados para trabajar"); Villas at Parkside Partners, 701 F. Supp. en 839 (afirmando que "la Ordenanza 2952 pone condiciones de residencia con respecto a viviendas de alquiler").

58. Ver id.

59. Ver Farmers Branch, Tex., Ordinance 2903 (12 de Mayo del 2007) (adoptada por el voto de toda la ciudad por un margen de 2-1 para sustituir a la Ordenanza 2892).

60. Ver Villas at Parkside Partners v. City of Farmers Branch, 577 F. Supp.2d 858, 874-75 (N.D. Tex. 2008). 
para la asistencia federal de vivienda. ${ }^{61}$ En la decisión inicial del 2008, el Tribunal del Distrito revocó la ordenanza basándose en el derecho de prioridad y en las garantías procesales. ${ }^{62}$ La ciudad intentó corregir las violaciones relacionadas con el principio de prioridad y las garantías de un proceso justo con una ordenanza de seguimiento, que además de tener un gran parecido a otras ordenanzas de otras localidades, no contiene ninguna referencia a la elegibilidad para subsidios de vivienda federal, incorpora mecanismos de revisión local y judicial y se remite a la ley federal para determinar el status de inmigrante autorizado. ${ }^{63}$

En casos sobre el principio de prioridad, los tribunales utilizan una presunción en contra de tal prioridad federal si consideran que el ámbito es uno "que los Estados han ocupado tradicionalmente." En Farmers Branch, sin embargo, el tribunal declaró que la ordenanza dependía suficientemente de las leyes de inmigración federal como para no merecer tal presunción. Consecuentemente el Tribunal de Distrito revocó la ordenanza en base al principio de prioridad en la materia. ${ }^{65}$ El tribunal concluyó que la Ordenanza, a propósito de las normas federales, "usó las clasificaciones para fines no autorizados o contemplados por la ley federal." "66 Finalmente el tribunal concluyó que la ciudad había impuesto restricciones en el alquiler basadas en las clasificaciones de inmigración para alterar directamente la capacidad de las personas en permanecer en el país, 0 , para decirlo de otra manera, las ordenanzas indebidamente afectaron la inmigración y no sólo a los inmigrantes. ${ }^{67}$

\section{Id. en 875-77.}

62. Ver Farmers Branch, Tex., Ordenanza 2952, § 26-79(B); 26-119 (2010) (indicando que el propósito de la ley era "para promulgar reglamentos que están en armonia con la ley federal de inmigración y que ayudan en su cumplimiento ... no ... de alterar, sustituir, interrumpir o interferir con el derecho federal de inmigración."); ver también Villas at Parkside Partners, 701 F. Supp. en 841 (enfocándose en la adopción de las definiciones federales, la deferencia a la determinación federal del estado de inmigración y la citación a la lucha contra portadores de disposiciones).

63. U.S. v. Locke, 529 U.S. 89, 108 (2000).

64. Ver Villas at Parkside Partners, 701 F. Supp. en 841 (citando Villas en Parkside Partners v. City of Farmers Branch, 577 F. Supp.2d 858, 866-67 (N.D. Tex. 2008)).

65. Id. en 855 .

66. Id. (citando De Canas, 424 U.S. en 358).

67. Ver Hazleton, Pa. Illegal Immigration Relief Act Ordinance (IIRAO) [Ordenanza del Acto de Alivio de Inmigración Ilegal], Ordenanza 2006-18, (2006) disponible en http://www.smalltowndefenders.com/090806/2006-

$18 \% 20 \_$Illegal\%20Alien\%20Immigration\%20Relief\% 20Act.pdf. Al igual que la ordenanza de Farmers Branch, IRAO incluye recitales con respecto a la autoridad de la ciudad para regular viviendas locales y el empleo en la medida en que se relaciona con la salud, la seguridad y el bienestar de los residentes de la ciudad. Id. en § 2; ver también Farmers Branch, Tex., Ordenanza 2903 (12 de mayo del 2007). 
Al igual que en Farmers Branch, la ciudad de Hazleton, Pensilvania también aprobó una ordenanza que regulaba a los inmigrantes no autorizados, aunque ésta fue más allá y también intentó sancionar a los empresarios por contratar a inmigrantes no autorizados a la vez que requería la implementación de E-Verify. ${ }^{68}$ La ordenanza también adoptó términos federales para la definición de "presencia legal" y requirió la verificación por parte del gobierno federal de la determinación final sobre la presencia autorizada. ${ }^{69}$ El Tribunal del Distrito de Pensilvania anuló la ordenanza en su totalidad por razones de prioridad; ${ }^{70}$ el Tribunal del Tercer Circuito confirmó la decisión. ${ }^{71}$

A pesar de que fue finalmente remitido para reconsideración, la decisión del Tercer Circuito en Hazleton podría haber sido vista como una reprimenda de parte del circuito dirigida a cualquier ley anti inmigración adoptada a nivel local. ${ }^{72}$ La decisión destacó favorablemente la decisión en Farmenrs Branch, y rechazó los resultados en Chicanos por la Causa, ${ }^{73}$ que requerían la implementación de E-Verify, y Valley Park, caso sobre una ordenanza similar donde el Tribunal había invalidado solo la parte relativa a

68. Ver Pa., Ordinance 2008-18 §3E (estableciendo que la definición de "trabajador ilegal" es una persona que no tiene el derecho legal o la autorización para trabajar debido a un impedimento en cualquiera de las disposiciones de la ley federal, estatal o local, incluyendo pero no limitado a un menor de edad descalificado por minoría de edad, o a un extranjero no autorizado para trabajar según lo definido por el Título 8 de los Estados Unidos, subdivisión 1324a(h)(3).

69. Ver Lozano, 496 F.Supp. 2d en 477.

70. Ver Hazleton, 620 F.3d en 170 (afirmando en parte y revocando en parte).

71. Ver id. Si bien la Corte Suprema de Justicia anuló y devolvió la decisión para su reconsideración, otras decisiones recientes que fueron devueltas para ser reconsideradas de manera similar, fueron posteriormente ratificadas por el Tercer Circuito. Ver, por ejemplo, Abu-Jamal v. Sec'y, Pen. Dept. of Corrs., 643 F.3d 370 (2011); Kindler v. Horn, 642 F.3d 398 (2011), afirmado en parte, revisado en parte U.S. v. Bennett, 427 Fed.Appx. 228 (3d Cir. C.A. 2011 ).

72. Ver Chicanos Por La Causa, Inc. v. Napolitano, 544 F.3d 976 (9th Cir. 2009) opinión modificada y caso sobreseido al ser negado en revisión, 558 F.3d 856 (9th Cir. 2009) afirmado sub nom. Whiting, 131 S. Ct. at 1968; Arizona Contractors Ass'n, Inc. v. Candelaria, 534 F. Supp. 2d 1036, 1046 (D. Ariz. 2008) afirmado sub nom. Chicanos Por La Causa, Inc.544 F.3d en 976 opinión modificada y caso sobreseido al ser negado en revisión, 558 F.3d 856 (9th Cir. 2009) afirmado sub nom. Whiting, $131 \mathrm{~S}$. Ct. en 1968 and afirmado sub nom. Chicanos Por La Causa, Inc. 558 F.3d en 856 afirmado sub nom. Whiting, 131 S. Ct. en 1968.

73. Hazleton, 620 F.3d at 216, 218 (señalando que "sin embargo, estas decisiones no proporcionan la debida importancia a los propósitos que llevaron a la decisión del Congreso para mantener el programa de E-Verify como un programa voluntario," que estas decisiones "probablemente aumenten la discriminación y contravendrian la ley de IRCA en ausencia de un componente de lucha contra la discriminación," y "menospreciaran el énfasis que el Congreso puso en la prevención de la discriminación ....”. 
los derechos de propiedad. ${ }^{74}$ Además, en el momento de decidir, el tribunal tenía la ventaja de contar con la decisión del Noveno Circuito de Chicanos por la Causa (reformada como en Whiting) en la mano.

El Tribunal del Tercer Circuito examinó de cerca la Cláusula Restrictiva a la hora de discutir el principio de prioridad expreso en el contexto de los empresarios, y también para prevenir la decisión en Whiting. Al final el tribunal sostuvo que la revocación de una licencia de negocio por la violación de la prohibición de contratar a inmigrantes no autorizados estaba dentro del ámbito de protección de la Cláusula de Salvedad. ${ }^{75}$ Sin embargo, en relación al principio de prioridad implícito, el tribunal sostuvo que la ordenanza perturbaba enormemente el delicado equilibrio entre la disuasión del empleo de inmigrantes no autorizados y la imposición de la carga a los empresarios y por lo tanto se presentó como un obstáculo para el cumplimiento de las regulaciones de las leyes federales de inmigración. ${ }^{76}$

Para concluir, el Tercer Circuito sostuvo que tanto la parte de la ordenanza relativa al empleo como la relativa al alquiler debían ser desplazadas. Las provisiones de los empresarios se revocaron debido a la teoría del principio de prioridad implícito. El tribunal declaró que la ciudad había creado un sistema inaceptable y alternativo de cumplimiento para los empresarios y terratenientes, que, si se permitiese podría resultar en un número aún mayor de estados y municipios implementando la aplicación de una legislación similar, que conduciría a un cúmulo cumplimiento para empresarios. ${ }^{77}$ La ciudad resultó aún peor en la parte de la ordenanza relativa al alquiler ya que el tribunal consideró que dicha parte estaba en

74. Ver id. en 238. El Tercer Circuito dio a notar argumentos "muy preocupantes" que el "Propósito Final de la Declaración" de la ordenanza no hizo referencia al tema de las licencias y por lo tanto las leyes deberían ser vistas como un "esquema destinado a regular la inmigración." Id. en 209 n.31.

75. Id. en 210-13 (tomando nota también de un menor número de garantias procesales para los que violan la ley desacuerdo a la ordenanza).

76. Ver id. en 213-216. En el contexto de las regulaciones del empleador, el Tercer Circuito señaló varios conflictos, incluyendo:

1) crear un mecanismo alternativo para la investigación de quejas,

2) un menor número de garantías procesales,

3) la eliminación efectiva del puerto seguro creado por la I-9 a través del uso mandatorio del E-Verify, y

4) aumentar las obligaciones para aquellos que emplean en más de un estado.

Id. (destacando que un mosaico de leyes como este "no podría estar en mayor conflicto con la intención del Congreso para la tramitación y cuidadosamente elaborado sistema de adjudicación de reducir al mínimo la carga impuesta a los empleadores") (citando Rowe v. N.H. Motor Transp. Ass'n, 552 U.S. $364,373(2008))$.

77. Hazleton, 620 F.3d en 219-20 (énfasis en el original). 
directa oposición a la decisión en De Canas, ya que iba dirigida específicamente a regular "quién puede vivir en Hazleton en base al estatus de inmigrante ...."78

Mientras estos dos casos se encuentran entre los más destacados de aquéllos en que los tribunales anularon las ordenanzas locales por razones de prioridad, no fueron los únicos. Una ley que obligaba a los empresarios a verificar la autorización de trabajo en contratos independientes, que va más allá del alcance de la IRCA, y que también creaba una acción legal contra los empresarios que contrataban a inmigrantes indocumentados, ${ }^{79}$ fue revocada por el Décimo Circuito también por motivos de prioridad ${ }^{80}$ y una ordenanza de vivienda similar a las ordenanzas de Hazleton fue anulada por razones de prioridad en California. ${ }^{81}$

\section{ii. Leyes y Ordenanzas Locales Confirmadas}

En contraste con las decisiones en Hazleton y Farmers Branch, Valley Park fue tratada de manera diferente-al menos en relación a la parte correspondiente a los empresarios y a E-Verify, y representa un caso en el que una ordenanza local sobre el tema de la inmigración no fue anulada. ${ }^{82}$ En Valley Park, después de reconocer brevemente que la presunción en contra del principio de prioridad era aplicable al caso, el Tribunal resolvió rápidamente la cuestión sobre la prioridad expresa basándose principalmente en el lenguaje del estatuto y en la Cláusula de Salvedad. ${ }^{83}$ De la misma manera, el Tribunal discutió en forma de sumario el argumento sobre la prioridad implícita en el ámbito, destacando que "requería poca discusión" ya que la Cláusula de Salvedad evidenciaba una voluntad a nivel federal de permitir ciertas regulaciones subfederales sobre

78. Una disposición similar se encuentra en la ley SB 1070. Ver Ariz. S.B. 1070.

79. Ver Chamber of Commerce of the U.S. v. Edmondson, 594 F.3d 742, 750 (10th Cir. 2010).

80. Ver Garret v. City of Escondido, 465 F.Supp.2d 1043, 1057 (S.D. Cal. 2006).

81. Ver Gray v. City of Valley Park, No. 4:07CV00881 ERW, 2008 WL 294294, en *14 (E.D. Mo. 2008). Aunque Valley Park tenía inicialmente una ordenanza sobre alquiler, además de una ordenanza relacionada al empleo, la ciudad derogo la ordenanza sobre alquiler voluntariamente tan sólo unos meses después de su adopción. Ver id. en *1; ver también Valley Park, Mo. Mun. Ordinance 1721 (febrero 2007). En el caso de Valley Park, el Tribunal de Distrito "notó respetuosamente que la decisión de [Hazleton] no [es] vinculante," y por lo tanto, evaluaría de forma independiente los argumentos del principio de prioridad. Ver City of Valley Park, 2008 WL 294294, en * 10 n. 13.

82. Ver City of Valley Park, 2008 WL 294294, en *10 (afirmando que "la pregunta de la Corte es solamente si la ordenanza es una ley de licencias u otra ley similar".).

83. Ver id. en *13. 
licencias. ${ }^{84}$ Sin embargo, aunque el Tribunal examinó de manera superficial las dos primeras acciones sobre el principio de prioridad, sí que brindó un extensivo comentario sobre el principio de prioridad implícito en caso de conflicto. ${ }^{85}$ Donde el Tribunal del Distrito y el Tercer Circuito en Hazleton habían encontrado numerosos problemas en relación a la posibilidad de conflicto entre la ley federal y lenguajes muy similares en ordenanzas locales independientes, ${ }^{86}$ el Tribunal de Valley Park no encontró ninguna, aunque lo hizo de una manera bastante indirecta. ${ }^{87}$

A diferencia de lo que puede ser considerado más como conflictos menores, el área de mayor preocupación en términos del principio de prioridad en Valley Park debió de ser el desplazamiento del balance de las cargas establecidas por la IRCA y extendidas por la IIRIRA. En ese aspecto muy poco ha sido dicho en el contexto del principio de prioridad ya que el Tribunal trató esas preocupaciones como basadas en igualdad de protección, y sobre esa discriminación, "poca discusión es necesaria." ${ }^{88} \mathrm{El}$ Tribunal sin embargo le dio más importancia al análisis de las provisiones de E-Verify, y su decisión fue subsecuentemente validada en Whiting. ${ }^{89}$

\section{Más allá de E-Verify y el Empleo: S.B. 1070 y sus Imitadores}

En la discusión local/nacional, ninguna ley ha causado mayores rumores que la S.B. 1070 de Arizona. Dicha ley, ${ }^{90}$ inicialmente impuesta por el Tribunal del Distrito de los EEUU, pretende expandir de manera importante el papel de los estados en materia de inmigración requiriendo que los agentes del orden locales participen con los oficiales federales, proveyendo a los individuos con una acción legal privada para impugnar al gobierno en el caso de no aplicar las leyes pro-restricción, creando crímenes estatales adicionales por la contratación (del empresario y el trabajador) y fallo de llevar a cabo los papeles de registro del inmigrante, incrementando las posibilidades de los agentes de determinar el estatus de inmigrante y

84. Verid. en *13-*19.

85. Ver supra notas 68-78.

86. Ver City Valley Park, 2008 WL 294294, en *13-*19 (discutiendo argumentos de derecho de adquisición preferente). En respuesta al supuesto conflicto entre la exención federal, que elimina a los trabajadores domésticos de la definición de empleado y la ordenanza de Valley Park, que posiblemente incluiría trabajador doméstico en su definición, el tribunal sostuvo que “ . . . la pequeña excepción que se ha creado no es suficiente para crear un conflicto entre la intención del Congreso y la ordenanza local." Id. en *14.

87. Verid. en * 19.

88. Ver id. en *16; ver también Whiting, $131 \mathrm{~S}$. Ct. en 1968.

89. Ariz. S.B. 1070.

90. Ver Arizona 703 F. Supp. 2d en 1008, afirmado 641 F.3d 339 (9th Cir. 2011). 
permitiendo arrestos sin orden del juez cuando exista razón suficiente para creer que el inmigrante está en EEUU de manera ilegal. ${ }^{91}$

El impacto potencial de la ley S.B. 1070 va más allá del ámbito de este artículo, sin embargo una breve mención es necesaria. Mientras las provisiones más ofensivas sobre inmigrantes fueron anuladas en base al principio de prioridad, los argumentos del gobierno federal al referirse a la ley se centraron en el principio implícito de prioridad en caso de conflicto y de ámbito en lugar de en el principio expreso. Mientras algunas de las provisiones prohibidas no llegan al nivel de regulación de inmigración que encontramos en De Canas, sí que se acercan al nivel de Hines en cuanto a su interferencia o conflicto con el comprensivo esquema federal de inmigración. ${ }^{92}$

Entonces, bajo la ley actual, asumiendo que el precedente en Hines continúe siendo válido, S.B. 1070 y leyes similares que traten de expandir la participación de estados y criminalizar comportamiento ya considerado bajo el marco de regulación federal deben continuar siendo anuladas. ${ }^{93} \mathrm{El}$ problema, claro esta, es que los estados continúan actuando a favor de la aplicación de estas leyes sin vigilancia alguna, y tampoco existen oportunidades compensatorias para los estados más simpatizantes. La trilogía de las decisiones del 2011 agudiza el enfoque del actual derecho sobre el principio de prioridad en la materia de inmigración, pero aún sigue dejando la balanza a favor de su aplicación.

\section{2011: La Corte Suprema Actúa}

Antes del verano de 2011, era suficientemente fácil reconocer los diferentes resultados surgidos a consecuencia de las continuas batallas sobre el principio de prioridad en Hazleton (Tercer Circuito), Farmers Branch (Quinto Circuito), Escondido (Noveno Circuito), Arizona (Noveno Circuito) y Oklahoma (Décimo Circuito) como el origen de la sana división que había en los circuitos. ${ }^{94}$ Pero, dadas las tres últimas acciones de la

91. Ver Arizona 703 F. Supp. 2d en 999.

92. De hecho, la nueva ley de Georgia ya ha sido ordenada por el gobierno federal. Ver Ga. Latino Alliance for Human Rights v. Deal, 793 F. Supp. 2d 1317, 1322 (N.D. Ga. 2011) (concediéndole al demandante un mandamiento judicial preliminar).

93. Ver Lozano, 620 F.3d en 170; Villas at Parkside Partners, 701 F. Supp. 2d en 835; Garret, 465 F.Supp.2d en 1043; Arizona, 641 F.3d en 339; Edmondson, 594 F.3d en 742.

94. Ver Regents of the Univ. of Cal., $131 \mathrm{~S}$. Ct. en 2961; Whiting, $131 \mathrm{~S}$. Ct. en 1970; Hazleton, Pa., 131 S. Ct. en 2958. 
Corte Suprema, al menos algunas de estas divisiones están siendo resueltas. $^{95}$

\section{a. Cámara de Comercio de los Estados Unidos v. Whiting}

Primero, debemos de comenzar con una discusión descriptiva de cualquier división que Whiting resuelve. Al reafirmar la decisión del Noveno Circuito por un margen de 5-3, ${ }^{96}$ el Tribunal reconoció el derecho de Arizona de promulgar leyes sobre licencias que, de no ser por la Cláusula de Salvedad, hubiesen sido desplazadas en base a la legislación federal de inmigración. ${ }^{97}$ No sorprendentemente, el Tribunal se basó en gran medida en el lenguaje de De Canas, el cual destacó que los "Estados poseen una amplia autoridad bajo sus poderes políticos para regular las relaciones de empleo para proteger a los trabajadores dentro del Estado," y que prevenir la contratación ilegal "está claramente dentro del conjunto de poderes políticos de [los estados] . ..."98

De cierta importancia para la decisión de Whiting es que Arizona delegó al gobierno federal la determinación de cuándo un inmigrante estaba autorizado para trabajar antes de imponer sanciones consistentes en la suspensión de licencias o su revocación, y adoptaron en buena medida la definición federal de "licencia" y defirieron a las determinaciones federales sobre el estatus de inmigración. ${ }^{99}$ Remarcando la definición de "licencia"100

95. Ver Whiting, 131 S. Ct. en 1968; ver también Nina Totenberd and Ari Shapiro, Kagan Recused From Pending Supreme Court Cases, NPR (29 de Septiembre del 2010), disponible en http://www.npr.org/templates/story/story.php?storyld=130205734 (declarando que J. Kagan se abstuvo de participar en la decisión dado su papel como procurador general antes de su confirmación).

96. Ver Whiting, $131 \mathrm{~S}$. Ct. en 1970 (afirmando que la ley federal de inmigración ahora tiene expresamente derecho de prioridad sobre sanciones civiles o penales impuestas a los empleadores aparte de las que son permitidas por la Cláusula Restrictiva); ver también 8 U.S.C. 8 U.S.C. $\S$ $1324 \mathrm{a}(\mathrm{h})(2)(2006)$.

97. Whiting, 131 S. Ct. en 1974 (citando De Canas, 424 U.S. en 356, 360.)

98. Ver ARIz. REV. STAT. ANN.\$ 23-211 (2007) (West) (definiendo a un "extranjero no autorizado" como "un extranjero que no tiene el derecho legal bajo la ley federal para trabajar en los Estados Unidos como esta descrito en el titulo 8 del United States Code $§ 1324 a(h)(3) ") ;$ ARIZ. REV. STAT. ANN $\S 23-214$ (2007) (West); Whiting, 131 S. Ct. en 1973, 1975, $1982-84$.

99. Ver Whiting, $131 \mathrm{~S}$. Ct. en 1978 (afirmando que "la ley de Arizona define la" licencia "como" forma de cualquier permiso emitido por una agencia, certificado, aprobación, registro, carta u otra forma similar de autorización que se requiere por ley y que es emitido por cualquier agencia a los fines de operar un negocio en el estado." (citando ARIZ. REV. STAT, § 23-211(9)(a)). Además "esta definición se asimila en gran medida a la definición de" licencia "que el Congreso estipula en la Ley de Procedimiento Administrativo."); ver también 5 U.S.C. § 551(8) ("licencia' incluye la totalidad o una parte de un permiso de una agencia, certificado, aprobación, registro, carta, la pertenencia, la exención legal u otro tipo de permiso"). 
al interpretar la Cláusula de Salvedad, el Tribunal consideró que el estatuto de Arizona, de "sentido común," entraba dentro de esa definición. No inesperadamente, el Tribunal afirmó la decisión del Noveno Circuito de que el derecho sobre licencias encajaba dentro de la Cláusula Restrictiva. De todas formas, el tema más importante ha sido siempre si, incluso asumiendo la aplicabilidad de la Cláusula Restrictiva, habría fundamentos jurídicos para aplicar el principio de prioridad implícito dado el poder pleno sobre inmigración, como algunos analistas han argumentado, y la necesidad de uniformidad. ${ }^{101}$

En gran parte desestimando los argumentos a cerca de que el Congreso había conseguido un balance adecuado al redactar leyes de inmigración, ${ }^{102}$ el Tribunal emitió una decisión inequívoca al menos en relación al derecho de licencias y la implementación obligatoria de E-Verify. ${ }^{103}$ Utilizando análisis similares a los del Noveno Circuito, el Tribunal razonó que el simple hecho de que el Congreso proveyera un mecanismo para cierta regulación sub-federal en la forma de leyes de concesión de licencias va en contra de cualquier argumento sobre que el Congreso intenta llenar el campo completo de la inmigración. ${ }^{104}$ Además, el Tribunal sorteó el problema del pleno poder al construir la ley como una "regulación [de] negocios entre estados mediante la concesión de licencias," un área tradicional de regulación estatal. ${ }^{105}$ Con ese marco adoptado, el análisis sobre el principio de prioridad era sencillo. El Tribunal decidió que el Congreso había proveído expresamente a los Estados con un mecanismo para imponer sanciones mediante las leyes de concesión de licencias, que el estatuto de Arizona actuó complementando en vez de suplementando las prohibiciones federales sobre la contratación de inmigrantes no autorizados, $y$, por eso, no existía un conflicto expreso o implícito. ${ }^{106}$

El análisis de E-Verify fue también sencillo y siguió las premisas de que:

100. Ver Whiting, $131 \mathrm{~S}$. Ct. en 1990 (Breyer, J., opinión disidente) (oponiendose a la pérdida de "un esquema de ejecución centralizada"); ver tambien Pham, supra nota 33.

101. Id. en 1990-92 (Breyer, J., opinion disidente) (resaltando las múltiples formas en que los objetivos contrapuestos, para hacer cumplir las prohibiciones contra la contratación de empleados no autorizados y la limitación de las obligaciones para los empleadores, están equilibrados).

102. Id. en 1972.

103. Id. en 1971.

104. Id. en 1983.

105. Ver Whiting, $131 \mathrm{~S}$. Ct. en 1971 (sef̂alando que los peticionarioss que pretendan demostrar que hay derecho de prioridad basándose en la teoria de que hay conflicto entre propósitos, deben cumplir un umbral alto para demosrarlo).

106. Id. en 1975 ("El [Secretario de Seguridad Nacional] no puede requerir a cualquier persona o entidad participar en un programa piloto.") (citando IIRIRA § 402(a), 110 Stat. 3009-656). 
1) La ley federal sólo prohibía un mandato federal de el programa de E-Verify; ${ }^{107}$

2) E-Verify fue creado por el Congreso para el uso de todos los estados; ${ }^{108}$

3) Posteriormente Arizona adoptó su propio mandato de EVerify de manera voluntaria. ${ }^{09}$

Aunque los argumentos sobre la eficacia de E-Verify son legítimos, ${ }^{110}$ carecen de fundamentos legales para impugnar la ley. La única cuestión a resolver, la cual el Tribunal respondió afirmativamente, fue el hecho de saber si las acciones de Arizona estaban dentro del ámbito aceptable de actividad. $^{111}$

Consecuentemente, en relación al principio de prioridad, Whiting establece dos precedentes importantes. Primero, la regulación subfederal dentro de la esfera tradicionalmente reservada a los estados, relativa a la concesión de licencias, y no reguladora de la inmigración en el sentido descrito en De Canas, es válida según la Cláusula de Salvedad. Segundo, la legislación subfederal exigiendo el uso de E-Verify, siempre y cuando no se aleje de los estándares y definiciones federales, no supone conflicto alguno y es asimismo aplicable. La implicación de ambas conclusiones serán discutidas después en la Parte III.

\section{b. Mártinez v. Regentes de la Universidad de California}

Mientras que el pronunciamiento de la Corte Suprema al negar certiorari en Martínez fue mucho mas breve que la decisión en Whiting, el

107. Id. en 1986 (en el 2003, el Congreso requirió que el programa E-Verify se ponga a disposición en los 50 estados) (citando 117 Stat. 1944; IIRIRA $\S 401$ (c)(1), 110 Stat. 3009-656).

108. Id. en 1986 (en el 2003, el Congreso requirió que el programa E-Verify se ponga a disposición en los 50 estados) (citando 117 Stat. 1944; IIRIRA $\S 401$ (c)(1), 110 Stat. 3009-656).

109. Id. en 23 (señalando que el gobierno federal había argumentado en el 2008, con respecto a la ley de Arizona, que "esto está permitido porque el estado de Arizona no es el Secretario de Seguridad Nacional") (énfasis en el original) (citando Memorando de respuesta del Demandado en apoyo de su propuesta de sentencia sumaria(se omite la cita)).

110. Ver E-Verify Statistics and Reports [Estadísticas e Informes del E-Verify], USCIS disponible en http://www.uscis.gov/portal/site/uscis/menuitem. eb1d4c2a3e5b9ac89243c6a7543f6dla/?vgnextoid=7c579589cdb76210VgnVCM100000b92ca60aRCR D\&vgnextc hannel=7c579589cdb76210VgnVCM100000b92ca60aRCRD (modificado por ultima vez el 7 de junio del 2011) (ultima visita el 15 de junio del 2011) (mostrando que en el año fiscal 2010 casi 47.000 personas con autorización de trabajo (ciudadanos y no ciudadanos por igual) recibieron una confirmación de autorización de trabajo incorrecta inicialmente).

111. Ver Whiting, $131 \mathrm{~S}$. Ct en 1987. 
impacto en el actual marco del principio de prioridad es considerable. ${ }^{112}$ Como se ha mencionado anteriormente, en Martínez ${ }^{113}$ el Estado de California redactó una ley que pretendía otorgar a cualquier estudiante, sin tener en consideración su estatus de inmigración, y que cumpliera con ciertos requisitos de asistencia y graduación, la oportunidad de asistir a una de las universidades públicas de California pagando la matrícula de la universidad. ${ }^{114}$

Aunque sea obvio a primera vista, hay una gran similitud entre Martínez y Whiting. Ambos casos contienen una estrecha interpretación sobre la doctrina del principio de prioridad cuando se enfrentan al lenguaje específico del estatuto. En Whiting, la Cláusula de Salvedad fue una cláusula tradicional reservando a los estados la habilidad de actuar de forma proscrita y estrecha. Mientras los Jueces discrepantes optan por una definición más limitada de "licencia" y una correspondiente aplicación más amplia de la doctrina referente al principio de prioridad, la Corte no fue influenciada por lo que vio como una solución de lenguaje sencilla. ${ }^{115} \mathrm{De}$ la misma manera, en Martínez, aunque irónicamente después de que las posiciones de las partes hubiesen sido revocadas, ${ }^{116}$ la Corte Suprema de California adoptó una lectura literal del lenguaje del estatuto federal que también tendía a limitar el alcance de los argumentos sobre el principio de prioridad.

Como se ha señalado, el derecho federal prohíbe a los estados otorgar beneficios a los inmigrantes sin papeles "en base a la residencia de éstos dentro del estado . . p para cualquier beneficio educativo postsecundario . . ." a menos que el ciudadano o residente de ese estado sea elegible para los mencionados beneficios sin tener en cuenta el estatus de residencia. ${ }^{17}$ Con tal de evitar esa prohibición, California condicionó la concesión de la matrícula de la universidad al hecho de que los estudiantes hubiesen asistido al colegio en California por al menos tres años y haberse graduado

112. Ver Regents of the Univ. of Cal., 131 S. Ct. en 2961.

113. Ver Martinez, 241 P.3d en 861.

114. Id. (citando CAL. EdUC. CODE [CÓdIGO DE EDUCACIÓN DE CALIFORNIA] $\S 68130.5$ (West 2001)).

115. Ver Whiting, $131 \mathrm{~S}$. Ct en 1988.

116. En Martínez, las partes que buscaban dejar sin efecto la ley que concedía beneficios ilegales a los inmigrantes no autorizados abogaron por una interpretación más amplia del lenguaje de la ley y recurrieron a los argumentos de la historia legislativa y finalidad de la ley. Ver Martinez, 214 P.3d en 855, 862-866. Por el contrario, la parte a favor de que la aplicación de la ley de Arizona fuera declarada invalida debido al principio de prioridad, fue quien se se baso en los argumentos de la historia y el propósito. Ver id.

117. Ver 8 U.S.C. 1623(a). 
en un colegio de California. ${ }^{118}$ En esencia, el estado usa la localización de la escuela superior como sustituto para determinar la residencia del estudiante a la hora de decidir quién debe recibir los beneficios de la matrícula de la universidad del estado. ${ }^{119}$ La Corte Suprema de California destacó una correlación entre la población estudiantil que probablemente serían elegibles en base al estatuto y aquellos que eran realmente residentes del estado, pero consideró que dado que los dos criterios son claramente distintos, la sustitución era apropiada. ${ }^{120}$

Dado que la letra del estatuto federal sólo prohíbe beneficios basados en la residencia del individuo, en base a un análisis sobre el lenguaje literal del estatuto, el Derecho de California no contradice tal prohibición y por lo tanto evita el principio de prioridad expreso. ${ }^{121}$ Empezando con el enfoque sobre el lenguaje literal del estatuto ${ }^{122}$ y el análisis sobre el principio de prioridad anterior, el argumento sobre la prioridad en Martinez casi refleja el de Whiting respecto a la Supremacía del derecho federal en los Estados Unidos en materia de inmigración. ${ }^{123}$ Trabajando a través del resto del esquema del estándar de prioridad: conflicto implícito (y/u obstáculo) y la prioridad del ámbito, la discusión siguió una dirección similar.

Sin embargo, en Martinez la Corte Suprema de California fue explícita en decir que era evidente que el estado estaba intentando promulgar legislación para dar un beneficio no permitido a inmigrantes ilegales. ${ }^{124}$ Incluso asumiendo que esa proposición fuera correcta, la corte destacó en relación al análisis de prioridad expreso, "nada hay de ilegal en el intento de

118. Ver CAL. EdUC. CODE [CÓdIGO DE EdUCACIón DE CALIFORNIA] § 68130.5 (West 2001).

119. Ver id. La Corte de Apelaciones de California estimo que la autorización legal era inapropiada como un requisito residencial de facto. Ver Martinez v. Regents of Univ. of Cal., 83 Cal.Rptr.3d 518, 531, 540 (Cal. Ct. App. 2008) revisión otorgada y opinión sobreseida, 198 P.3d 1 (Cal. 2008) y revisado bajo el nombre. Martinez, 241 P.3d en 855.

120. Ver Martinez, 241 P.3d en 864 (negando de forma explícita los criterios como el requisito de residencia de sustituto o de facto, esta limitación de responsabilidad no era necesaria dado el análisis de la corte que la ley federal en cuestión prohíbe beneficios basados en la residencia, "no algún tipo de sustituto de la residencia ....").

121. Ver id. en 863-64 ((observando que el estatuto no puede ser leído lógicamente como dependiendo en la residencia como un criterio ya que "muchas personas que no son residentes pueden calificar para ello") (sin énfasis en el original)). Además, el tribunal señaló que si la intención del Congreso hubiera sido prohibir los beneficios de educación superior para los inmigrantes no autorizados, lo podría haber hecho con facilidad. Id. en 864.

122. Ver id. en 864-66 (discutiendo los antecedentes legislativos potencialmente adverso que se opone al idioma actual de la ley).

123. Ver id. en 861; ver también Whiting 131 S. Ct. en 1975 . En Martinez, sin embargo, la corte se negó discutir la cuestión del principio de prioridad, señalando que era inmaterial para el resultado final. Ver Martinez, 241 P.3d en 862.

124. Ver Martinez, 241 P.3d en 866. 
evitar [la prohibición federal en beneficios postsecundarios]." ${ }^{25}$ Sin embargo, es una pregunta requerida saber si ese intento puede crear un conflicto u obstáculo en la "conclusión y ejecución de los propósitos y objetivos del Congreso."

El problema legal enfrentado por los oponentes de la ley es que la ley federal sí incluye una provisión expresa del principio de prioridad, la cual no cubría la conducta/ley en cuestión. Como la Corte Suprema ha mantenido que la inclusión de una cláusula expresa del principio de prioridad implica que el Congreso no tenía la intención de cubrir asuntos fuera de su ámbito de decisión, ${ }^{127}$ el argumento sobre el intento de la legislatura del estado, apropiado o no, es legalmente insuficiente siempre y cuando sus acciones caigan fuera del ámbito de la cláusula de prioridad. ${ }^{128}$ Además, cualquier argumento sobre la prioridad del ámbito es igualmente debilitado por una cláusula limitada de prioridad expresa dado que cualquier límite implica una ventana para acciones del estado que a la vez sugiere que el Congreso no quería "ocupar completamente el campo." 129

\section{c. Hazleton v. Pensilvania}

Hazleton, el caso final de la Corte Suprema en 2011 de la trilogía sobre el principio de prioridad, fue dictado el mismo día que Martínez. ${ }^{130}$ En una opinión de dos oraciones, la Corte otorgó certiorari y después revocó la decisión del Tercer Circuito y la envió para nueva consideración a la luz de su decisión en Whiting. ${ }^{131}$ Mientras que no se puede decir mucho sobre la decisión en este momento, parece que va a ser cada vez más difícil para los oponentes de la parte de la ordenanza de Hazleton relativa al empleo ser nuevamente victoriosos teniendo en cuenta la decisión en Whiting. De hecho, lo máximo que se puede deducir es que a la Corte le gustaria ver que el marco del principio de prioridad continúa siendo unificado en relación a las provisiones sobre empleo en base a la Cláusula Restrictiva y el uso de E-Verify. Probablemente también se vea emerger un consenso coherente a nivel de los tribunales del circuito sobre las ordenanzas de alquiler ya que ninguna jurisdicción ha mantenido ese tipo de regulación subfederal.

125. Id.

126. Hines 312 U.S. en 67 (citando Cf. Savage v. Jones, 225 U.S. 501,533 (1912)).

127. Ver Freightliner Corp. v. Myrick, 514 U.S. 280, 288 (1995).

128. Ver Martinez, 241 P.3d en 868.

129. Id.

130. Ver Hazleton, 620 F.3d en 170.

131. Ver Regents of the Univ. of Cal, 131 S. Ct. en 2961 
De hecho, esta dicotomía entre empleo y vivienda parece ser precisamente lo que la Corte ha establecido en este periodo. Parece ser que la Corte, a la hora de aclarar el ámbito y aplicabilidad de la Cláusula de Salvedad y los limites del principio de prioridad implícito, ha vuelto a un análisis más robusto de De Canas que discutiblemente reduce los fundamentos para el principio de prioridad implícito a la hora de analizar las regulaciones subfederales que directa o indirectamente afectan la inmigración. Más problemático para muchas localidades es probablemente la pregunta "[q]ué viene después?" $\mathrm{Al}$ negarse a proveer una respuesta definitiva sobre las ordenanzas de vivienda, y dado el hecho de que muchas localidades promulgan ordenanzas de empleo y vivienda, es previsible que la mayor parte del litigio quede pendiente en este contexto.

\section{ACTA DEL EsTADo Y LocALIDAD}

A pesar de que Arizona y ahora Utah, ${ }^{132}$ Georgia, ${ }^{133}$ y Alabama ${ }^{134}$ (entre otros) han sido los protagonistas, muchos otros estados y ciudades a través del país han estado ocupados considerando y haciendo leyes relacionadas a la inmigración. ${ }^{135} \mathrm{El}$ estado de Nebraska, geográficamente en el centro del pais, ${ }^{136}$ no ha sido una excepción. Debido a los objetivos de este artículo, dos leyes principales serán mencionadas, una modificada a nivel estatal de la S.B. 1070 de Arizona, ${ }^{137}$ y la ordenanza local adoptada

132. Utah Illegal Immigration Enforcement Act [Ley de Ejecución de Inmigración Ilegal de Utah], Utah H.B. 497.

133. H.B. 7089, 113th Gen. Assemb. (Fla. 2011).

134. H.B. 56, Reg. Sess. (Ala. 2011).

135. Ver Liz Goodwin, Report: 25 States Considering Arizona-Style Immigration Laws [Reporte: 25 Estados Considerando el Estilo de Arizona para sus Leyes de Inmigración], YaHOO! NEwS, 28 de octubre del 2010, http://news.yahoo.com/s/yblog_upshot/20101028/ us_yblog_upshot/report-25-states-are-considering-arizona-style-immigration-laws (última visita el 17 de marzo del 2012); John Miller, Twenty Other States Considering Copying Arizona Immigration Law [Veinte Otros Estados Considerando Copiar a las Leyes de Inmigración de Arizona], HougHINGTON POST , 25 de junio del 2010, disponible en http://www.huffingtonpost.com/2010/06/25/twenty-otherstates-consi_n_626095.html (ultima visita el 17 de marzo de 2012).

136. A través del análisis amateur con un viejo atlante de Rand-McNally, este autor ha determinado que el estado de Nebraska, por ejemplo, se encuentra aproximadamente a 1.350 millas de la costa oeste, $1.000 \mathrm{~km}$ de la frontera sur, 1.250 millas de la costa este, y sólo un 400 kilómetros de la frontera norte, haciendo el Estado de Nebraska unos de los estados, o si no el estado, más lejos de cualquier frontera internacional.

137. Proyecto de Ley Legislativa 48, este proyecto de ley, que fue inspirado por el proyecto de ley 1070 del senato de Arizona, no pudo avanzar las discusiones de el comité, por muchas razones, y lo menos important fuero las cuestionabilidad de su validez, y el costo necesario para la defensa de esta ley. Ver Neb. L.B. 48 [Proyecto de Ley Legislativa 48 de Nebraska]; ver generalmente Kevin O'Hanlon, Arizona-Style Immigration Measured Vetted in Overflow Public Hearing [Inmigración al 
por referéndum y modelado en la ordenanza de Hazleton prohibiendo el empleo de inmigrantes no autorizados y requiriendo "permisos de ocupación" para alquilar espacios de viviendas. ${ }^{138}$

Antes de discutir estas leyes y en parte a explicar su ímpetus, me gustaria destacar algunos factores sobre el estado de Nebraska y el pueblo de Fremont, donde se redactó la ordenanza, en la Nebraska rural. La población hispana de Nebraska es de aproximadamente 8.4\% (comparada con el $15.8 \%$ de la población general de los Estados Unidos); sin embargo, aunque la población hispánica de Nebraska está por debajo de la media del país, el Censo del 2010 indicó un crecimiento del $77 \%$ en una término de diez años. ${ }^{139}$ Mostrando aún más el cambio demográfico tenemos el de los niños inmigrantes en 1990 comparado con el periodo 2005-2006..$^{140} \mathrm{El}$ porcentaje de los niños inmigrantes en 2005-2006 es de $11 \%$, cuatro veces mayor al por ciento que en $1990 .^{141}$

Parece que no es sólo el tamaño de la población lo que ha provocado la legislación reciente, sino la velocidad a la que esta está creciendo. Este problema, la pregunta de si las poblaciones extranjeras han llegado más rápidamente de lo que la sociedad cree que se puedan asimilar en los Estados Unidos, ha atormentado al país desde su inicio hace 300 millones

Estilo de Arizona fue Analizada en Audiencias Püblicas Llenas a Capacidad], LinCOLN JOURNAL STAR, (2 de marzo del 2011), disponible en http:/journalstar.com/news/unicameral/article_717ddfa5-d5555550-be0d-81d3271 ffff8.html (ultima visita el 17 de marzo de 2012).

138. Ver Fremont, Neb., Ordinance 5165 [Ordenancia 5165 de Fermont, Nebraska] (21 de junio de 2010); Cindy Gonzalez, Fremont in the Spotlight [Fermont en Foco de Atención], OMAHA WORLD HERALD, 30 de marzo del 2012, disponible en http://www.omaha.com/article/20100530/ NEWS01/705309883 (ultima visita el 17 de marzo de 2012) (explicando que inicialmente la Ordenancita de Fermont fue derrotada en el nivel de consejo de la ciudad y que, siguiendo otras derrotas, los que apoyaron la Ordenancita pudieron obtener el numero de signaturas suficiente para que el asunte se decidiera por referendo); Cindy Gonzalez, Fremont Weighs Tax Hike to Defend Law - The Public Can Comment on a Plan to Pay for Costs Related to the Immigration Ordinance [Fremont Pesa el Aumento de Impuestos para la Defensa de la Ley - El Público Puede Comentar Sobre el Plan de Pagar los Costos Relacionados con la Ordenanza de Inmigración], OMAHA WORLD HERALD, 23 de augusto del 2010, en 01B (explicando que los residentes aprobaron la Ordenanza mayoritariamente por un voto de dos a uno, sabiendo que un voto afirmativo iba a resultar en un aumento de impuestos a la propiedad de aproximadamente $18 \%$ ).

139. Ver Sharon R. Ennis et al., The Hispanic Population 2010 [La Poblacion Hispana del 2010], U.S. CENSUS BUREAU (2010), disponeble en www.census.gov/prod/cen2010/briefs/c2010br04.pdf (ultima visita el 16 de marzo de 2012).

140. Ver The Henry J. Kaiser Family Found., Nebraska: Immigrants [Nebraska: Inmigrantes], STATE HEALTH FACTS, disponible en http:/www.statehealthfacts.org/profileind.jsp?cat= $1 \&$ sub $=157 \&$ rgn $=29$.

141. Id. 
de personas. ${ }^{142}$ Desde preocupaciones coloniales específicas, a través del trato de esclavos africanos importados y seguido por las leyes de exclusión de chinos, al sistema de cuota de inmigración, los Estados Unidos ha estado siempre preocupado con el hecho de tener demasiados inmigrantes, y estas preocupaciones han llevado a un incremento de la legislación en estos periodos, y el resultado en Nebraska en el 2011 no ha sido distinto. ${ }^{143}$

Teniendo en cuenta tanto la base de la población inmigrante como el índice de crecimiento, no es sorprendente que la comunidad de inmigrantes en Nebraska sea de una importancia económica significativa, especialmente en ciertos sectores. En general, los inmigrantes proveen aproximadamente un $7 \%$ de la mano de obra Nebraska ${ }^{144}$ (incluyendo un aplastador $80 \%$ en los empleos de la producción de carne). ${ }^{145}$ Tal vez la estadística más importante, todavía no mencionada, es el índice de desempleo. Nebraska, desde Junio del 2011, tiene el segundo puesto más bajo en el índice de desempleo del país, ${ }^{146}$ y algunos pueden considerar que ya está en su

142. Ver, por ejemplo, Ralph Frasca, "To Rescue the Germans out of Sauer's Hands": Benjamin Franklin's German-Language Printing Partnerships ["Para Rescatar a los Alemanes de las Manos de Sauer": Asociación de Publicaciones de el Idioma Alemán de Benjamin Franklin], 12 PA. MAGAZINE OF HISTORY \& BIOGRAPHY 329 (1997). En 1790, la población de los Estados Unidos era aproximadamente 3.9 millones, comparado con la población del 2011 que era aproximadamente 307 millones. Ver Population: 1790-1990 [La Población de los Estados Unidos entre 1790-1990], U.S. CENSUS BUREAU, tabla 4, http://www.census.gov/population/censusdata/table-4.pdf (última visita el 18 de marzo de 2012). En 1760 la población de los Estados Unidos era aproximadamente 1.6 millones. En ese momento, Benjamín Franklin escribió acerca de los inmigrantes alemanes, "[s]ino transferimos la corriente de importación desde esta colonia a otra, el número de inmigrantes será mucho más que nosotros, todas las ventajas que tenemos no podrá (en mi opinión) preservar nuestro idioma, y incluso, has nuestro gobiemo estará peligro." Ver Frasca, supra nota 144 en 343 (citando una carta de Benjamin Franklin a Peter Collinson (9 de mayo del 1753) en The Papers of Benjamin Franklin [Los Papeles de Benjamin Franklin], 4:484-85 (New Haven 1959)).

143. Daniel Kanstroom, Deportation Nation [Nación De DePORTaCión] 91-107, 219220 (Harvard Univ. Press 2007).

144. Ver Selected Characteristics of Native and Foreign-Born Population 2009 [Características Selectas de la Población Indigena y de Extranjeros 2009], U.S. CENSUS BUREAU, http://factfinder.census.gov/servlet/STTable?_bm=y\&-context=st\&-

qr_name $=$ ACS_2009_1YR_G00_S0501\&-ds_name $=$ ACS_2009_1YR_G00_\&-CONTEXT $=$ st\&tree id $=307 \&$-redoLog=false \&-geo_id=04000US31 \&-format $=\&$-_lang=en (ultima visita el 29 de junio del 2011).

145. Ver Christopher S. Decker et al., Nebraska's Immigrant Population Economic and Fiscal Impacts [Impacto Económico y Fiscal de la Populación de Inmigrantes en Nebraska], 2008 UNIV. OF NEB. 1, disponible en http://www.unomaha.edu/ollas/econ\%20im\%20report/econimpact.pdf (última visita el 17 de marzo de 2012). Importantemente, los inmigrantes también son neutrales fiscalmente (y de hecho ligeramente positivo) en Nebraska, en el sentido que ellos contribuya más de lo que reciben en beneficios patrocinados por el gobierno, incluidos los servicios de educación y salud. Id. en 22.

146. Ver Regional and State Employment and Unemployment Summary May 2011, U.S. BUREAU OF LABOR STATISTICS, disponible en http:/www.bls.gov/news.release/laus.nr0.htm 
máxima contratación. ${ }^{147}$ Nebraska también esta lidiando con otros factores demográficos interesantes tales como migración neta negativa del estado y una amenazante escasez de trabajo durante las próximas décadas. ${ }^{148}$

Adicionalmente, Fremont (y el Condado de Dodge del cual Fremont representa un $70 \%$ de toda la población del condado) es muy representativo en términos de las dinámicas mencionadas anteriormente. ${ }^{149}$ Por ejemplo, desde el 2000 al 2010 la población Hispana o Latina del condado se duplicó más del doble, ${ }^{150}$ y la población total Hispana o Latina del condado ahora está sobre el $10 \%$ de la población total del condado. ${ }^{151}$ Además, este crecimiento ocurrió en el momento en que el crecimiento total del condado, en términos de población, era solo un $1.5 \% .^{152}$

Es en contra de este fondo demográfico que la legislación estatal fue introducida en Nebraska en el 2011 para criminalizar el empleo de inmigrantes no autorizados en los Estados Unidos, el fallo de registrar o procesar una tarjeta de registro de extranjeros, y albergar con conocimiento o de manera imprudente a inmmigrantes no autorizados. ${ }^{153}$ También hubo leyes para promover a todos los condados y comunidades de Nebraska a entrar en el $287(\mathrm{~g})$ y/o Programas de Seguridad Comunitaria, ${ }^{154}$ expandir el

(modificado por última vez el 24 de enero de 2012) (última visita el 17 de marzo de 2012). Nebraska's unemployment rate of $4.1 \%$ trails only North Dakota. Id.

147. Ver 15 U.S.C. $\S 1022 \mathrm{a} ; 15$ U.S.C. $\S \S 3101$ et al. (mostrando que, aunque hay discusiones en cuál es la mejor definición de beneficios de empleo completo, el Congreso lo ha definido como un cuatro por ciento).

148. Ver Steve Carlson et al., Brain Drain in Nebraska: What do the Data Show? [Fuga de Cerebros en Nebraska: ¿Qué es lo que Muestran los Datos?], 63 Bus. in Neb. 1, 1-3 (2008) (explicando que la inminente falta de obra, aunque no es específico a Nebraska, podría llegar a ser especialmente problemático para los estados que ya están cerca a empleo total).

149. Ver Fremont (city), Nebraska, U.S. CENSUS BUREAU, http:/quickfacts. census.gov/qfd/states/31/31053.html (modificado por última vez el 31 de enero de 2012).

150. Ennis et al., supra nota 141.

151. Ver Profile of General Population and Housing Characteristics: 2010 [Perfil de la Población General y Caracteristicas de Vivienda del 2010], U.S. CENSUS BUREAU, http:/factfinder2.census.gov/faces/tableservices/jsf/pages/productview.xhtml?pid=DEC_10_DP_ DPDP1\&prodType=table (ultima visita el 18 de marzo del 2012).

152. Ver Dodge County, Nebraska, U.S. CENSUS BUREAU, http:/quickfacts. census.gov/qfd/states/31/31053.html (modificado por última vez el 31 de enero del 2012).

153. VER Neb. L.B. 48 [Proyecto de Ley 48 de Nebraska] (relacionado a la aprobación de la Ley de Ejecución de la Inmigración Ilegal); ver también Paul Hammel, Nebraska Legislature - Debate on Illegal Immigrants is Shelved [Asamblea Legislativa de Nebraska - El Debate Sobre los Inmigrantes Ilegales se Pospone], Omaha World Herald, 9 de marzo del 2011, en 4B (observando que esta y todas las demás propuestas relacionadas con la inmigración fracasaron de avanzar afuera del comité para pasar un año conduciendo un estudio preliminar sobre el tema).

154. L.R. 28, 102d Leg., 1st Sess. (Neb. 2011). 
uso obligatorio de E-Verify, ${ }^{155}$ y eliminar la beca del estado a inmigrantes no autorizados que cumplían con los requisitos estatutarios. ${ }^{156}$

Aunque no sea un problema en Nebraska tal y como la ley L.B. 48 y las otras propuestas de inmigración habían anticipado, el año de moratoria impuesto por el comité mientras estudia el "Compromiso de Utah", aprobado recientemente, podría ver los proyectos de ley reincorporados en el 2013. Igual que en Fremont, aunque la ordenanza fue adoptada, nunca fue aplicada. ${ }^{157}$ En el caso de Fremont, puede ser que la decisión de la Corte en Hazleton y Whiting haya tenido un impacto significativo. ${ }^{158}$ A menos que el Tercer Circuito vaya a reafirmar su decisión original en su totalidad, parece que al menos la parte de la ordenanza referida al empleo puede sobrevivir un desafio legal. ${ }^{159}$

Si eso ocurriera, el panorama político de Nebraska con respecto a las ordenanzas relacionadas con la inmigración sería uno propio de un estado donde las políticas de inmigración a nivel local y estatal continuarían siendo fomentadas, esta vez sin la discusión sobre la potencial constitucionalidad de las leyes y por tanto, una amenaza menos creíble del proceso disuasivo. En términos de relaciones raciales y el bienestar económico, dicha situación parece problemática dado algunos problemas recientes en las relaciones de comunidad. ${ }^{160}$ Sin embargo, la decisión

\section{Ver Neb. L.B. 569.}

156. VER L.B. 657 [Proyecto de Ley 657], 102d Leg., 1st Sess. (Neb. 2011) (suspendido por tiempo indefinido). No todas las regulaciones que fueron propuestas relacionas a inmigración eran "anti-inmigrante", como el proyecto de ley (aunque no fue promulgada) que restablecer la cobertura de atención prenatal para todas las mujeres pobres (incluyendo los inmigrantes sin documentos), ese benefició se elimino con una legislación aprobada en el 2010. Ver L.B. 599 [Proyecto de Ley 599], 102d Leg., I st Sess. (Neb. 2010) (en relación con el programa de asistencia médica para modificar el estatuto de Nebraska $\S \S 4-110$ y 68-901).

157. Ver Cindy Gonzalez, Fremont suspends Immigration Ordinance Until Suits are Resolved [Fremont Suspende la Ordenanza de Inmigración hasta que Resuelvan las Demandas contra la Ordenanza], OMAHA WORLD HERALD, 28 de Julio del 2010, en 04B (señalando que siguiendo la aprobación de la ordenanza por medio de referendo, el concejo municipal voto a favor de suspender la ejecución de la ordenanza debido a la amenaza de una orden restrictiva).

158. Ver Keller v. City of Fermont, Nos. 8:10-cv-0270-LSC-FG3, 4:10-cv-3140-LSC-FG3, 2011 WL 41902, en *1 (D.Neb. 2011); Ver también Fremont, Neb., Ordinance [Ordenancita de Fremont, Nebraska] 5165.

159. Ver Fremont, Neb., Ordinance [Ordenancita de Fremont, Nebraska] $5165 \S 5$ (a)-(h).

160. Ver, por ejemplo, Michael Kelly, Prejudice Spoils a Night of Triump [El Prejuicio Arruina una Noche de Triunfo], OMAHA WORLD HERALD, el 20 de mayo del 2010, en 02B (discutiendo el campeonato estatal de futbol de la escuela secundaria donde los estudiantes tiraron "tarjetas verdes" en el terreno para demostrar su oposición de la ubicación de la escuela en una zona principalmente hispánica). Específicamente en Fermont, el miedo y el prejuicio contra los inmigrantes incrementaron por causa del uso constante del español en la YMCA o en Wal-Mart. Hasta las personas que han sido residentes por un mucho tiempo han sentido el aumento de amargura en la comunidad, como el 
legislativa de Nebraska de posponer cualquier decisión sobre leyes relacionadas con la inmigración en el 2011 con la intención de poder estudiar el enfoque de Utah también sugiere una voluntad de considerar un ámbito más amplio de legislación, un ámbito que tal vez no sea posible a menos que se lleven a cabo algunos cambios en el debate del principio de prioridad.

\section{PRoponiendo Un TRINQueTe BILATERAL}

En este artículo propongo un enfoque más extenso en el tema del principio de prioridad, con algunos límites críticos, en relación a las regulaciones de inmigración subfederales. Mientras que un enfoque neutral más amplio tiene el beneficio potencial de permitir a las localidades la habilidad de implementar las leyes sobre un espectro más amplio, también tiene la desventaja potencial de ser usado como un vehículo para avanzar leyes discriminatorias tales como las invalidadas en California y en Texas lidiando con la educación de niños inmigrantes no autorizados, ${ }^{161} \mathrm{o}$ una ley más extensa sobre el crimen/aplicación como las que se promulgaron recientemente en Arizona, Georgia y Alabama. ${ }^{162}$

Los comentarios sobre este asunto han incrementado rápidamente y han sido diversos. ${ }^{163}$ En un lado del debate, el Profesor Pham ha optado por

vandalismo del negocio de uno de los residentes antiguos. Ver Leslie Reed, June 21 Vote on Ordinance - Fremont is Still Torn on Immigrants [El Voto del 21 de junio sobre la Ordenanza - Fremont Sigue Dividido sobre los Inmigrantes], OMAHA WORLD HERALD, 10 de junio del 2010, en 01A; Paul Hammel, Fremont Officials are Concerned About Tensions - The City Council is Considering a Resolution to Discourage "Divisive Behavior" in Regard to the Immigration Ordinance [Oficiales de Fremont Están Preocupados por Causa de la Tensiones - El Concejo Municipal está Considerando una Resolución para Desaminar "Conducta Divisible" con respecto a la ordenancita de inmigración], OMAHA WORLD HERALD, 10 de septiembre del 2010, en 3B (citando 65 acusaciones de hostigamiento relacionada con la raza desde la elección especial que aprobó la ordenanza).

161. Ver, por ejemplo, League of United Latin Am. Citizens v. Wilson, 908 F. Supp. 755, 764 (C.D. Cal. 1995); Plyler v. Doe, 457 U.S. 189, 189 (1982). En ambos casos, la corte anuló las leyes que pretendían restringir el acceso a la educación primaria y secundaria.

162. Ver supra notas $135-237 \mathrm{y}$ el texto adjunto.

163. Ver, por ejemplo, Keith Aoki \& John Shuford, Welcome to Amerizona - Immigrants Out!: Assessing "Dystopian Dreams" and "Usable Futures" of Immigration Reform, and Considering Whether "Immigration Regionalism" is an Idea Whose Time has Come [Bienvenidos a Amerizona Fuera con los Immirantes!: Evaluación de "Suenos Distopicos" y "Futuros Utilizables" de la Reforma Inmigratoria, y Considerando si "El Regionalismo de Inmigración" es una Idea Cuyo Tiempo ha Llegado], 38 FORDHAM URB. L.J. 1 (2010) (proponiendo leyes regionales de inmigración similares al sistema canadiense que permite las regiones cierta autonomía en la determinación de temas de inmigración); Gabriel J. Chin et al., A Legal Labyrinth: Issues Raised by Arizona Senate Bill 1070 [Un Laberinto Jurídico: Temas Planteadas Por el Proyecto de Ley 1070 del Senato de Arizona], 25 Geo. IMMIGR. L.J. 47 (2010) (Analizando temas del principio de prioridad relacionados con el Proyecto de ley 1070 del Senato de Arizona); D. Fan, Post-Racial Proxies: Resurgent State and Local Anti-"Alien" 
un rol federal exclusivo en el debate sobre la inmigración basándose en los poderes constitucionales y plenarios. ${ }^{164}$ Este argumento fue usado con éxito en la decisión del Tercer Circuito en Hazleton, y al menos parcialmente en Arizona, pero las decisiones más recientes de la Corte estipulan de forma clara algún tipo de rol para la legislación subfederal. Antes de las decisiones del 2011, este enfoque fue quizá favorecido por su habilidad para mantener una apariencia de balance entre la legislación a favor de los inmigrantes y también a favor de su ejecución. ${ }^{165}$

El Profesor Rodríguez, por otra parte, ha argumentado que un marco uniforme no es ni requerido por la ley ni necesariamente beneficioso en un clima donde hay poca o más bien ninguna acción legislativa a nivel federal. ${ }^{166}$ El Profesor Rodríguez, mientras reconoce que cierto control es necesario para prevenir a los estados y localidades de que produzcan externalidades negativas, también se decanta por una "cuenta funcional," donde las cortes y legisladores por igual interpreten reclamaciones del principio de prioridad de una manera más limitada para poder proveer un "dialogo continuo ... a nivel de los estados y locales." 167 El problema con este enfoque es que, aunque es neutral, el tenor actual del debate sobre inmigración y el dramático desvío del derecho actual hacia la aplicación, tiene la oportunidad de convertir esto en un mecanismo en gran parte antiinmigrante.

Laws and Unity-Rebuilding Frames for Antidiscrimination Values [Elementos Pos Raciales: Surgen de Nuevo Leyes Estatales y Locales Contra "Inmigrantes" y Reconstrucción de Unidades y Valores Antidiscriminatorios] , 32 CARDOzo L.R. 905 (2011); Pratheepan Gulasekaram, Sub-national Immigration Regulation and the Pursuit of Cultural Cohesion [Reglamento de Inmigración Subnacional y la Búsqueda de la Cohesión Cultural], 77 U. CIN. L. REV. 1441 (2009); Michael A. Olivas, Immigration-Related State and Local Ordinances: Preemption, Prejudice, and the Proper Role for Enforcement [Ordenancitas Estatales y Locales Relacionada a Inmigración], 2007 U. CHI. LEGAL. F. 27 (2007); Pham, supra nota 33 (argumentando que el mandato constitucional de las leyes uniformes de inmigración resultan en el sobreseimiento de las leyes subfederales que de lo contrario resultaría en una maraña de conflictos entre las leyes); Rodriguez, supra nota 39 (argumentando a favor de un papel funcional para los estados y municipios en la legislación relacionada con la inmigración y al mismo tiempo oponiéndose a la noción de exclusividad federal en inmigración); Peter J. Spiro, Learning to Live with Immigration Federalism [Aprendiendo a Vivir con el Federalismo de Inmigración], 29 CONN. L. REV. 1627 (1997); Rick Su, A Localist Reading of Local Immigration Regulations [Una Lectura Localista de los Reglamentos Locales de Inmigración] 86 N.C. L. REV. 1619 (2008) (argumentando que los estados y municipios deben de involucrarse en alguna manera en la regulación de inmigración).

164. Ver Pham, supra nota 33, en 1003.

165. Ver id.

166. Ver Rodríguez, supra nota 39, en 571-72 (declarando que una legislación uniforme es a la misma vez "dificil de lograr, [y] también es muchas veces contraproducente.").

167. Id. en 596. En la vigilancia de algunas de las externalidades negativas, el profesor Rodríguez también se basaría en otras protecciones legales y constitucionales como las Enmiendas I y XIV y otras leyes de propietarios. Id. en 572. 
El gran impulso subfederal para regular la inmigración ha continuado con la propuesta casi diaria de leyes más duras. Aún así, lo que el Profesor Rodríguez finalmente ha concluido tiene mérito; que la normalización de la autoridad de inmigración es necesaria para permitir la coexistencia de las regulaciones sobre inmigración del estado y las federales a través del uso de la doctrina estándar del principio de prioridad. ${ }^{168}$ En gran medida este Artículo, en principio, está de acuerdo con estos argumentos, que parecen ir más allá del marco de De Canas. ${ }^{169}$ La prueba que provee el Profesor Rodríguez, lidiando específicamente con el conflicto del principio de prioridad en caso de conflicto (aunque aparentemente aplicable por analogía al principio de prioridad de ámbito) es si las leyes subfederales "impiden" la aplicación federal del derecho de inmigración, ${ }^{170}$ y no si existe "tensión con los objetivos federales." 171 Sin embargo, dado las tendencias de los estatutos de anti-inmigración propuestos, este Artículo propone que la normalización, para poder actuar efectivamente como "normalización," debe tomar su forma como un mecanismo muy pro-inmigrante (no necesariamente pro-inmigración) para proveer un nivel legislativo en que los estados y localidades puedan actuar. ${ }^{172}$

La implementación de este tipo de doctrina de prioridad tiene el beneficio general de la neutralidad federal. El límite en la legislación subfederal a favor de la aplicación permanecería estático, mientras que la legislación pro-inmigrante permitiría a los estados embarcarse en programas hechos para sus regiones específicas, dentro de unos límites establecidos. La neutralidad prevista permitiría a los estados promulgar más leyes relacionadas con la inmigración que incluirían probablemente las porciones aprobadas judicialmente de la S.B. 1070, así como leyes sobre asilo e incluso leyes relacionadas con el empleo. Este tipo de enfoque, aplicado correctamente, puede alcanzar esa porción de la ley de Utah que busca otorgar permisos de trabajo dados por el estado a la vez que estaría limitando las disposiciones más perjudiciales.

Tal y como está, el análisis del principio de prioridad está en un cruce de caminos. Sin una relajación ni reinterpretación de la doctrina de

168. Ver Rodríguez, supra nota 39, en 610-11, 620, 622-23 (establecimiento un marco para la implementación del proceso de normalización que requeriría un cambio de la doctrina del principio de prioridad y también el uso de presunciones contra el principio de prioridad).

169. Id. en 624 (argumentando que las regulaciones de propietarios sólo tangencialmente se refiere a la eliminación o otras funciones de ICE y por lo tanto no deben ser considerada como regulaciones de inmigración).

170. Id. en 626 .

171. Id. en 610-11.

172. Id. en 617 . 
prioridad, el sistema actual permite a los estados poco espacio para la indulgencia en términos de permitir la presencia de inmigrantes no autorizados, mientras permitiéndoles una gran libertad para incrementar las medidas de aplicación ya sea de manera conjunta con el gobierno federal o a través de leyes unilaterales de concesión de "licencias." Dado que $D e$ Canas parece haber resucitado y que el Congreso ha proveído expresamente parauna regulación subfederal de inmigración anti-inmigrante, este Artículo cuestiona si puede ser que no halla espacio para esta tercera opción.

Aceptando Whiting como el actual estado del derecho de prioridad en relación a la inmigración (y sabiendo que la decisión de Arizona podría alterar fundamentalmente el enfoque de De Canas y/o Hines), podría parecer que un contrapeso a favor de la legislación subfederal proinmigrante debería ser permitido para que los estados puedan promulgar leyes que puedan satisfacer la prioridad y otros argumentos federales. $\mathrm{Si}$ determinadas medidas controvertidas tales como la revocación de licencias, implementación obligatoria de E-Verify y licencias de ocupación son permitidas, entonces porque no permitir (o promover) también leyes de licencias de conducir subfederales, ${ }^{173}$ permisos de trabajo emitidos localmente, leyes como el "Mini-Dream Act," o leyes de asilo?

Así que la pregunta entonces es, ¿existe una distinción legítima que se pueda hacer entre leyes que suponen una invasión aceptable en el marco tradicional de inmigración federal mientras no se vaya tan lejos como para abrir las compuertas y permitir que leyes claramente discriminatorias, como la Propuesta 187, desencadenen una carrera hacia el abismo entre los estados? ${ }^{174}$ Yo creo que la respuesta es un sí. La base para la legislación a favor de la aplicación fue establecida principalmente mediante la interpretación de la Cláusula Restrictiva. Sin embargo, para la legislación subfederal pro-inmigrante, la pregunta es más dificil ya que no hay una Cláusula Restrictiva para proveer a los estados y localidades con la flexibilidad legislativa requerida.

Claramente el Santo Grial para los defensores del aumento de inmigración sería la autorización del estado para su presencia y empleo.

173. Ver Real ID Act [Ley de Identificación Autentica], Pub. L. No.109-13, 49 U.S.C. 30301 (2005) § 202(a)(1) (explicando que, aunque no está prohibido por la ley federal, la Ley de Identificación Autentica motiva los Estados que hagan sus licencias mas seguras. El fracaso de no ofrecer esa seguridad podría tener la ramificación de invadir la licencia para abordar aviones o tener acceso a otras instalaciones federales).

174. Ver, Rodríguez, supra nota 39, en 640 n.303 (citando Peter H. Schuck, Same Federal State-Developments in Immigration Law [Algunos Desarrollos Federales y Estatales en las Leyes de Inmigración], 58 N.Y.U. Ann. Surv. Am. L. 387, 389 (2002)) (indicando que ninguna carrera hacia el fondo ocurrió en el año 1996 cuando los estados se les permitió prohibir los beneficios públicos en IIRIRA § 553). 
Bajo el marco legal actual (que hasta recientemente parecía poco probable que vaya a ser sometido a un cambio dramático en su estructura en un futuro cercano), visas emitidas por el estado son una imposibilidad como una regulación directa de inmigración. Mientras que otra alternativa, la de permisos de empleo autorizados por el estado, ha sido sugerida antes pero sin éxito, ${ }^{175}$ este Artículo incluiría unos permisos de empleo emitidos por el estado bajo la rúbrica de acciones aceptables por el estado a la hora de permitir legislación subfederal pro-inmigrante.

La clave para una presencia exitosa en los Estados Unidos de inmigrantes no autorizados es el acceso. Los inmigrantes necesitan acceso a vivienda, transporte, empleo, educación y servicios públicos (en el sentido de acceso a la protección de policías, al departamento de incendios y al sistema legal). Negar estos accesos a cualquiera de estas categorías incrementa la probabilidad de que los inmigrantes no autorizados sean presos y sujetos a victimización. De estas cinco categorías, sólo la educación ha sido decidida a favor de los inmigrantes, ${ }^{176}$ mientras las leyes subfederales han atacado consistentemente las otras cuatro. ${ }^{177}$ Con el fin de permitir leyes de contrapeso, de alguna manera los estados deberían poder legislar con gran discreción en el tema de dichas limitaciones.

Un posible mecanismo legal para lograr dicho resultado es eliminando la presunción de prioridad y motivar un sistema neutral estatal/federal de inmigrantes. ${ }^{178}$ Como ya ha sido reconocido, dicho enfoque podría permitir una legislación discriminatoria inaceptable si no se establecieran ciertos límites. ${ }^{179}$ El mecanismo propuesto, quizás más sencillo de implementar en la teoría y de utilidad limitada, sería coger prestado el concepto de aprobación expresa de la Cláusula Restrictiva para aplicarlo a la legislación pro-inmigrante - reconociendo que para que funcione como se espera, nuevo lenguaje es requerido en la Ley de Inmigración y Nacionalidad (INA) ${ }^{180}$ Además del problema inevitable de promulgar dicho lenguaje,

175. Ver Bill Ong Hing, NAFTA, Globalization and Mexican Migrants [NAFTA, Globalización y Migrantes Mexicanos], 5 J.L. ECON. \& POL'Y 87, 166-67 (2009) (señalando que en 2010, Iowa estableció una comisión para mejorar la economía del estado, y unas de sus recomendaciones era "Designar para lowa una 'zona empresarial de inmigrantes' y buscar una excepción de las cuotas de inmigración federales.").

176. Ver, por ejemplo, Plyler, 457 U.S. en 189.

177. A pesar que no hay leyes que han tratado de negar protección policial a los inmigrantes sin documentos, leyes que requieren a los agentes del orden público que determinen el estatus migratorio de cualquier persona con quien legalmente entran en contacto, claramente impide el ejercicio de ese derecho.

178. Ver supra nota $174 \mathrm{y}$ el texto adjunto.

179. Ver supra nota 178.

180. Ver supra notas 179-80. 
podría decirse que existe apoyo judicial para este enfoque ya que fue el enfoque que la Corte implícitamente siguió en Martínez. ${ }^{181}$

La Corte, usando el canon del lenguaje literal, dijo que el estatuto prohibía un beneficio muy específico - uno otorgado sólo en relación a la residencia, y por lo tanto, todo tipo de conducta sobre beneficios postsecundarios se salvó. ${ }^{182}$ De la misma forma, con beneficios del estado, los estados retienen el derecho de otorgar beneficios públicos a inmigrantes ilegales, y los casos han seguido utilizando un lenguaje sencillo para validar leyes posteriores otorgando beneficios incluso cuando los estatutos no hacian referencia a la $\S 411$ de la Ley de Responsabilidad Personal y Oportunidad de Reconciliación del Trabajo del 1996 (PROWRA). ${ }^{183}$ Estos son dos ejemplos muy conocidos, aunque limitados, donde en ambos casos el poder judicial ha inferido una especie de Cláusula de Restrictiva invertida.

Refiriéndonos otra vez a las otras categorías de acceso necesarias para inmigrantes, hay también un lenguaje legislativo expreso que técnicamente permite a los estados continuar otorgando las licencias de conducir (aunque dichas licencias no permiten para viajar entre estados), ${ }^{184}$ al igual que estatutos de asilo que discutiblemente no son tan amplios como para incluir el alquiler o la venta de las viviendas a inmigrantes ilegales (aunque son suficientemente amplios como para incluir políticas de asilo). ${ }^{185} \mathrm{La}$ fuerza de estos estatutos, sin embargo, no se asemeja a la de la Cláusula Restrictiva en que requieren lecturas casi híper-técnicas para poder contemplar los beneficios de los inmigrantes. ${ }^{186}$ Además, en ambos casos existen serias consecuencias por el fallo de cumplir con el deseado esquema

181. Ver Martinez, 241 P.3d en 855.

182. Ver id. en 855,865 .

183. Personal Responsibility and Work Opportunity Reconciliation Act of 1996, 42 U.S.C. $\S$ 1305 (2006).

184. Ver Real ID Act [Ley de Identificación Autentica], Pub. L. No. 109-13, 49 U.S.C. 30301 (2005) § 202(a)(I). Ha habido muchos comentarios excelentes sobre la readaptación de las licencias de conducir a actuar como una tarjeta de identificación nacional. Ver, por ejemplo, Kevin R. Johnson, Driver's Licenses and Undocumented Immigrants: The Future of Civil Rights Law? [Licencias de Conducir y Los Inmigrantes Indocumentados: El Futuro de la Ley de Derechos Civiles?], 5 NEv. L.J. 213, 221 (2004).

185. Ver 8 U.S.C. $\$ 1324$ (declarando que albergar de una forma ilegal debe de requerir saber o temerariamente ignorar si el individuo está presente legalmente); Ver, por ejemplo, Merkt, 794 F.2d en 950 (sosteniendo convicciones de encubrimiento cuando los acusados exigían que solo estaban dando "santuario" basadas en las creencias religiosas). El problema con alquiler o la venta de casas se puede crear con la práctica, que ahora es omnipresente, de utilizar los números de seguridad social para conducir una verificación de crédito. Número de seguro social incorrecto o ningún número, junto con otros factores, pueden elevarse al nivel de "ignorar temerariamente."

186. Ver supra notas 202-03 y el texto adjunto. 
federal. ${ }^{187}$ Más importante aún, no existe una interpretación disponible de la Cláusula Restrictiva inversa en relación a las provisiones de empleo proinmigrantes, derecho más importante a los ojos de los inmigrantes ilegales, y el área donde los estados están más limitados. ${ }^{188}$ Sencillamente, como era el propósito del Congreso, no hay lectura, literal o de otra manera, que permita a los estados autorizar permisos de trabajo sin una modificación significante de la ley actual. ${ }^{189}$

El propósito de este artículo, pues, es defender un enfoque entre teorías contrarias sobre las regulaciones de inmigración estales/federales concurrentes y la estructura actual que provee a los estados y localidades poca libertad para implementar legislación pro-inmigrante. ${ }^{190}$ Este artículo afirma que la manera más fácil para hacer esto es concediendo a los estados la capacidad expresa para regular las cinco áreas de acceso anteriores con respecto a legislación pro-inmigrante. ${ }^{191} \mathrm{La}$ propuesta es un poco desagradable en el sentido de que requiere acción del Congreso (o por lo

187. Ver Nicholas Confessore, After a Rough Start, Spitzer Rethinks His Ways [Después de un Comienzo Dificil, Spitzer Reconsidera su Forma de Ser], N.Y. TIMES, el 27 de noviembre de 2007, en Al (ilustrando que los ciudadanos de Nueva York se apresuraron a oponerse a las licencias de conducir que se había propuesto para todos, al enterarse de que sus licencias de conducir dejaría de ser válida para viajes aéreos). Anecdóticamente, el gobierno federal también ha enjuiciado a los propietarios por albergar, a alquilar un apartamento o casa. Ver, por ejemplo, Brandon Ortiz, Immigration Case Puts Focus on Landlords [Caso de Inmigración Centra la Atención en Los Propietarios], HoustoN Chronicle, el 25 de mayo de 2008, en A3; C.f. Recalde v. BAE Cleaners, Inc., 862 N.Y.S.2d 781 (N.Y. Sup. Ct. 2008). Además, las leyes federales, estatales y locales de la equidad de vivienda probablemente prohibirá toda discriminación por motivos de origen nacional, y por lo tanto las preguntas basada en la condición de ser extranjero puede resultar en violaciónes. Ver, por ejemplo, Sophie Marie Alcorn, Landlords Beware, You may be Renting Your own Room . . in Jail: Landlords Should not be Prosecuted for Harboring Aliens [Propietarios Tenga Cuidado, Usted Puede Alquilar su Propia Habitación . . en la Cárcel: Propietarios no Deben Ser Procesados por Albergar a los Extranjeros], 7 WASH. U. GLOBAL STUD. L. REV. 289, 304 (2008).

188. Ver 8 U.S.C. $\S 1324$ a (mostrando que el INA $\S 274 a$, implementado a través del IRCA, es amplio, estricto y comprensivo en su prohibición de emplear a inmigrantes no autorizados, a pesar de que tiene dos notables excepciones: el primero es para "el empleo ocasional de las personas que prestan servicio doméstico en una casa privada ya sea irregular, intermitente, o esporádicamente" y el segundo para los contratistas independientes); 8 U.S.C. $\S 1324 \mathrm{a}(\mathrm{a})(4)$ (prohibiendo la contratación de contratistas independientes "sabiendo que el extranjero es un extranjero sin autorización ....").

189. Ver supra nota 206 y el texto adjunto.

190. Ver infra nota 209 y el texto adjunto.

191. Educación y derechos de acceso merece una explicación adicional. El derecho a la educación se decidió principalmente por motivos de protección de la igualdad y por lo tanto una expansión de la capacidad de los Estados para legislar en esta área aún no autoriza a los Estados a anular las protecciones constitucionales. Ver Plyler, 457 U.S. en 210-14. En cuanto al acceso a los servicios públicos, la igualdad de protección también evita que los Estados nieguen a tales servicios, a través de tipos de iniciativas como Comunidades Seguras o la mayoría de las determinaciones legales de inmigración (con la posible excepción de los que podrian ocurrir en un ambiente escolar). 
menos, consentimiento anticipado por Utah en el contexto de empleo), pero es difícil concebir algún otro curso que permita a los estados la capacidad de regular asuntos de inmigración localmente que no sea únicamente de una manera pro-ejecución. ${ }^{192}$

¿Cómo entonces, podría este tipo de enfoque tratar con una ley como la S.B. 1070, Nebraska L.B. 48, la Ordenanza de Fremont 5165, o cualquier otras que se parezcan? En cuanto a la S.B. 1070, la cual ha sido litigada, la provisión que se mantuvo en el desafío continúa siendo válida. En cuanto a estas provisiones que fueron anuladas en base a la prioridad del ámbito, todas o casi todas continuarían siendo válidas bajo este nuevo esquema. De la misma manera, la Ordenanza de Fremont se enfrentaría al mismo resultado que en Hazleton - una orden de inadmisibilidad en relación a la parte de la ordenanza relacionada con los permisos de ocupación que regulan la inmigración, pero una aprobación de las provisiones de empleo. Uno puede ver que este enfoque no representa un cambio drástico del esquema de prioridad actual, o de la decisión en Hines que advierte fuertemente en contra del trato discriminatorio de inmigrantes autorizados para dejarlos "libres de la posibilidad de prácticas inquisitoriales y de vigilancia de la fuerza policial" ${ }^{" 193}$ como uno podría imaginar.

\section{CONCLUSIÓN}

No es frecuente que personas participando en un debate apasionado se encuentren en un momento de definición tan importante. Con el desorden en las leyes de prioridad en inmigración y los intentos subfederales para entrar en el campo, nosotros nos encontramos en dicha encrucijada. Mientras pro-reaccionistas pueden muy bien detestar la propuesta que este artículo sugiere, deberían pararse a reflexionar. Primero, en esos estados deseosos de tener menos inmigrantes, pocos mecanismos pueden ser más efectivos que el hecho de que una jurisdicción vecina proteja el acceso de los inmigrantes a las categorías anteriores. ${ }^{194}$ Segundo, la propuesta, aunque pro-inmigrante considerada en sí misma, es neutral en cuanto a anular esquemas legales y sólo busca un campo de juego en que los estados puedan actuar. Finalmente, esta propuesta contempla las tendencias anteriores en relación a las economías en depresión y legislaciones antiinmigrantes al igual que futuras tendencias de rechazo de tasas de nacimiento y escasez de trabajo en una manera en que el estándar actual de legislación no lo hace.

192. Ver supra notas 179-181.

193. Hines, 312 U.S. 74.

194. Profesor Spiro, más de una década, pidió un marco del principio de prioridad para permitir que los estados sean "laboratorios de las mejores prácticas." Ver Spiro supra nota 165, en 1636 n.34. 
Al final parece que el principio de supremacía federal y las leyes de regulación de inmigración están llegando a un cierre ya que a los estados y localidades les está siendo otorgado expresamente el derecho de regular. Nosotros deberíamos asegurar que la regulación no sea sólo de un lado, y deberíamos reconocer que es improbable que un trinquete unilateral a favor de la aplicación calme la animosidad de los debates actuales. Proveyendo a los estados y localidades con una voz y un rol, que sean pro-aplicación y pro-inmigrante a la vez, al igual que moldear la legislación actual puede ser la mejor manera de hacer evolucionar el debate cuando se enfrente con la posibilidad real de que ningún esfuerzo exhaustivo federal vaya a aparecer en un futuro cercano. 



\section{INTERNATIONAL LAW STUDENTS ASSOCIATION}

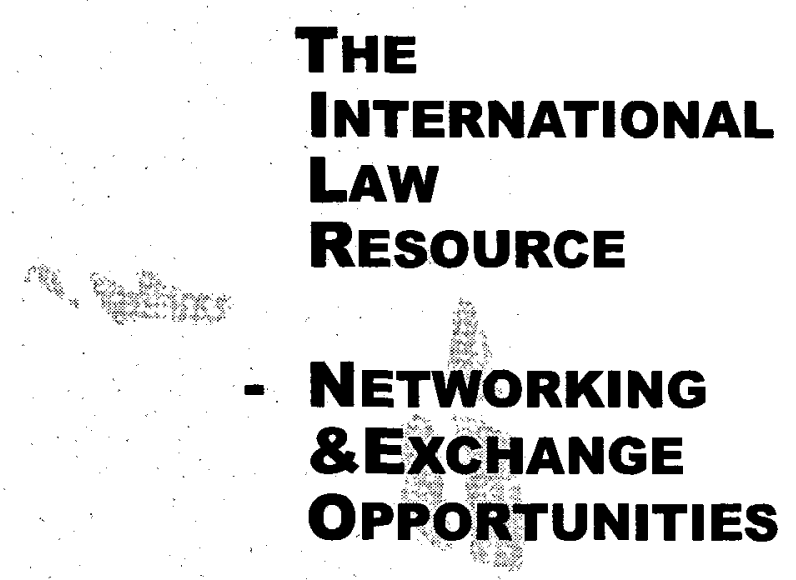

- EMPLOYMENT \&CAREER

DEVELOPMENT

ACADEMIC \& Professional DeVelopment
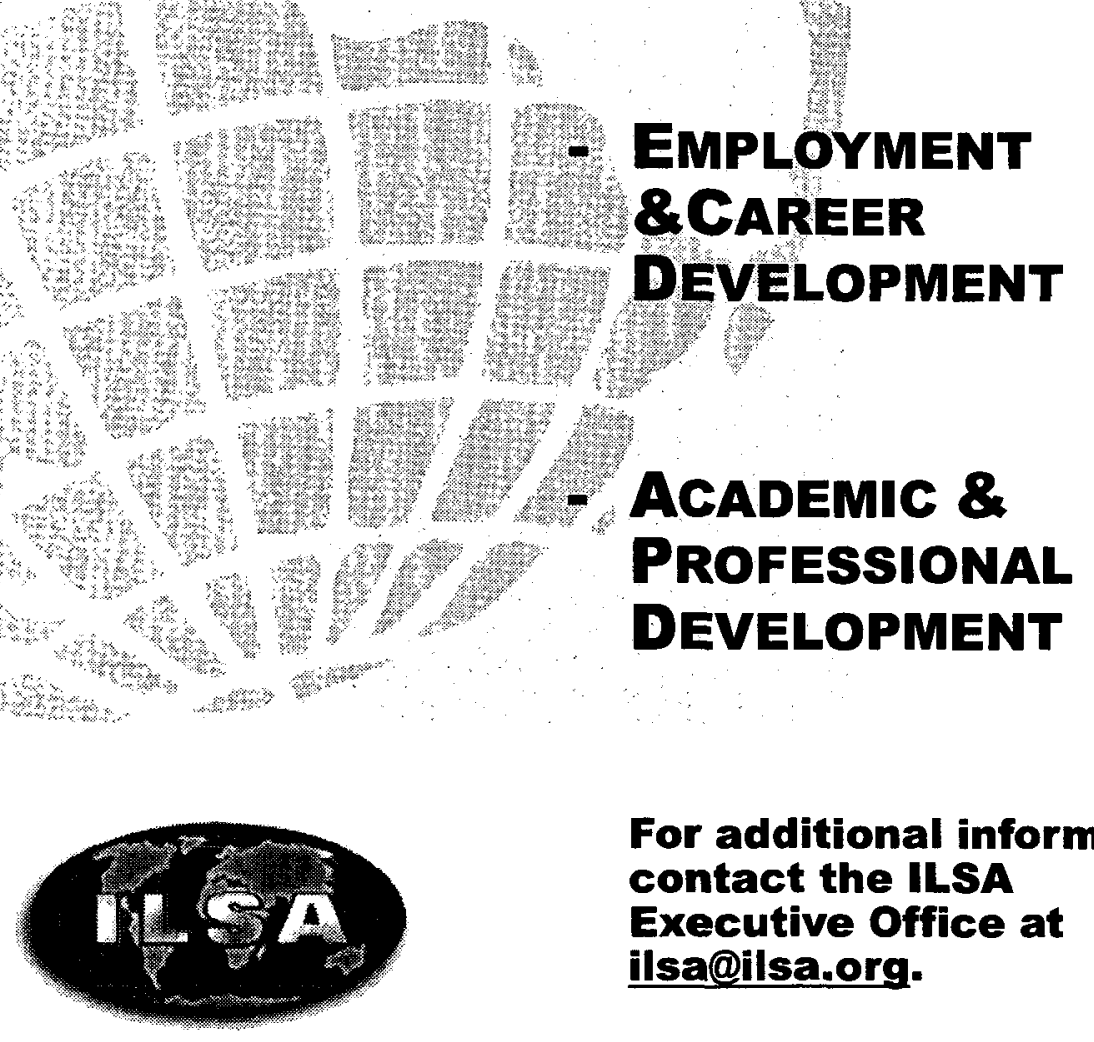

For additional information contact the ILSA

Executive Office at ilsa@ilsa.org. 



\section{AMERICAN BAR ASSOCIATION SECTION OF INTERNATIONAL LAW AND PRACTICE}

\section{http://www.abanet.org/intlaw/}

The ABA Section of International Law and Practice assists in international policy development, promoting the rule of law, and educating legal practitioners. Since 1933, it has been involved in a wide range of legal activities and international legal issues.

Committees within the Section of International Law and Practice include the following:

\section{Business Transactions \& Disputes Division \\ Business Regulation Division \\ Comparative Law Division \\ Public International Law Division \\ General Division}

Along with the International Law Association American Branch, the ABA Section of International Law and Practice co-sponsors the annual ILA Weekend in New York, New York, United States.

Selected ILA Weekend speeches, panel remarks, and other proceedings are published each year in the International Practitioners' Notebook, which is the annual spring issue of the ILSA Journal of International \& Comparative Law. Further information about the International Law Association and ILA Weekend is available at http://www.ambranch.org/.

Both law students and professionals in the field are welcome in the ABA Section of International Law and Practice. For membership information, visit http://www.abanet.org/about/membership.html. 



\title{
INTERNATIONAL LAW ASSOCIATION AMERICAN BRANCH
}

\author{
http://www.ambranch.org
}

The International Law Association (ILA), founded 1873 in Brussels, is a private international organization. It is a non-governmental association with United Nations consultative status. The ILA's biennial conference debates and proceedings have in many cases influenced the decisions of the United Nations General Assembly.

ILA members may belong to one of more than forty national ILA branches. The ILA's Secretary General of the Association maintains an office in London, England, and individual ILA members whose countries do not yet have ILA branches are members of the London "Headquarters." The ILA conducts international law study through committees. Committee members are specialists and prepare biennial conference reports under the supervision of the ILA's Director of Studies.

United States of America ILA Members enter the ILA by joining the Association's American Branch. American Branch members' ILA world conference reports appear in Proceedings of the American Branch.

The International Law Association American Branch sponsors the annual ILA Weekend in New York, New York, United States. Selected speeches, panel remarks, and other proceedings from the ILA Weekend are published each year in the International Practitioners' Notebook, the spring issue of the ILSA Journal of International \& Comparative Law.

More information and an online application for membership in the ILA American Branch are available on the ILA Website at http://www.ambranch.org/fee.htm. 


\section{INTER-AMERICAN CENTER FOR HUMAN RIGHTS NOVA SOUTHEASTERN UNIVERSITY, SHEPARD BROAD LAW CENTER \\ 3305 College AVE, Fort LAUdERDALE, FL 33314 http://www.nsulaw.nova.edu/iachr/index.cfm}

The Inter-American Center for Human Rights is a response to the profound need in South Florida for an organization that is committed to furthering the civil and human rights of our diverse communities and people. Recent projects are included on the IACHR website along with links.

\section{BOARD OF DIRECTORS}

Prof. James D. Wilets Chair

NSU Shepard Broad Law Center
Prof. Douglas L. Donoho

Vice Chair

NSU Shepard Broad Law Center
Prof. Charlene Smith

Executive Director

NSU Shepard Broad Law Center
Prof. Jane Cross

Project Director

NSU Shepard Broad Law Center
Prof. Florence Shu-Acquaye

Project Director

NSU Shepard Broad Law Center
Dean William E. Adams, Jr. Member

NSU Shepard Broad Law Center

Prof. Kathy Cerminara Member

NSU Shepard Broad Law Center NSU Shepard Broad Law Center

\section{STUDENT OFFICERS}

Melanie Malavé, Executive Student Director

Cosima Ortiz, Secretary

Ryan Tyre, Secretary

Kathryn Hamilton, Director of Public Relations and Advertising

Ethel Moreno, Director of Charitable Organization Status

Michael Rasmussen, Director of Legal Education

Molena Mompoint, Director of Community Partnerships

Laura Geddes Paez, Esq., Director of Legal Alumni Relations

Chasity Strachan, Director of Social Media

Katherine Hustis, Director of Volunteer Coordination

STUDENT STAFF

Adriana Barba

Kandia Batchelor

Marlon Bennett

Marisol Cruz

Gretel Echarte
Erin Fortin

Elena lovino

Karen Milia

Ethel Moreno

Victor Palacios
Monique Patterson

Carli Pierson

Jacob Pridgen

Adriana Torres 


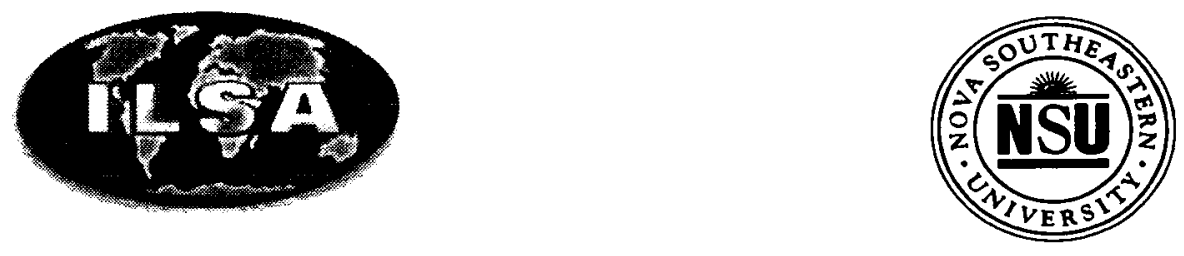

\section{ILSA Journal of International \& Comparative Law NOVA SOUTHEASTERN UNIVERSITY Shepard Broad Law Center}

The ILSA Journal of International \& Comparative Law is housed at Nova Southeastern University's Shepard Broad Law Center in Fort Lauderdale, Florida, and is an International Law Students Association publication. The ILSA Journal of International \& Comparative Law publishes three editions every year, including is a compilation of notes, comments, and essays; the International Practitioners' Notebook; and the Bilingual Edition.

Subscriptions: The subscription price is $\$ 30.00$ per volume per year for domestic subscribers and $\$ 35.00$ per volume per year for foreign subscribers. Subscriptions are renewed automatically unless the subscriber sends a timely notice of termination. All notifications of address changes should include the old and new addresses.

For subscriptions, please cut out the attached coupon and mail to:

Subscriptions Editor

ILSA Journal of International \& Comparative Law

Nova Southeastern University, Shepard Broad Law Center

3305 College Avenue

Ft. Lauderdale, FL 33314

Fax: (954) 262-3830

Name:

Address:

Payment is enclosed

Bill Me: 